Florida International University

FIU Digital Commons

FIU Electronic Theses and Dissertations

University Graduate School

6-28-2019

\title{
Ultra-Wideband Phased Arrays for Small Mobile Platforms
}

Alexander D. Johnson

ajohn326@fiu.edu

Follow this and additional works at: https://digitalcommons.fiu.edu/etd

Part of the Electrical and Computer Engineering Commons

\section{Recommended Citation}

Johnson, Alexander D., "Ultra-Wideband Phased Arrays for Small Mobile Platforms" (2019). FIU Electronic Theses and Dissertations. 4222.

https://digitalcommons.fiu.edu/etd/4222

This work is brought to you for free and open access by the University Graduate School at FIU Digital Commons. It has been accepted for inclusion in FIU Electronic Theses and Dissertations by an authorized administrator of FIU Digital Commons. For more information, please contact dcc@fiu.edu. 


\title{
FLORIDA INTERNATIONAL UNIVERSITY \\ Miami, Florida
}

\author{
A dissertation submitted in partial fulfillment of the \\ requirements for the degree of \\ DOCTOR OF PHILOSOPHY \\ in \\ ELECTRICAL AND COMPUTER ENGINEERING \\ by
}

Alexander Johnson 
To: Dean John L. Volakis

College of Engineering and Computing

This dissertation, written by Alexander Johnson, and entitled Ultra-Wideband Phased Arrays for Small Mobile Platforms, having been approved in respect to style and intellectual content, is referred to you for judgment.

We have read this dissertation and recommend that it be approved.

$\begin{array}{r}\hline \text { Elias A. Alwan } \\ \hline \text { Shubhendu Bhardwaj } \\ \hline \text { Arvind Agarwal } \\ \hline \text { John L. Volakis, Major Professor }\end{array}$

Date of Defense: June 28, 2019

The dissertation of Alexander Johnson is approved.

Dean John L. Volakis

College of Engineering and Computing

Andres G. Gil

Vice President for Research and Economic Development and Dean of the University Graduate School

Florida International University, 2019 
(c) Copyright 2019 by Alexander Johnson

All rights reserved. 


\title{
ABSTRACT OF THE DISSERTATION \\ ULTRA-WIDEBAND PHASED ARRAYS FOR SMALL MOBILE PLATFORMS by
}

\author{
Alexander Johnson \\ Florida International University, 2019 \\ Miami, Florida \\ Professor John L. Volakis, Major Professor
}

This dissertation presents the development of a new class of Ultra-Wideband (UWB) apertures for aerial applications by introducing designs with over 50:1 bandwidth and novel differential feeding approaches. Designs that enable vertical integration for flip-chip millimeter-wave (UWB) transceivers are presented for small aerial platforms. Specifically, a new scalable tightly coupled array is introduced with differential feeding for chip integration. This new class of beam-forming arrays are fabricated and experimentally tested for validation with operation from as low as 130 $\mathrm{MHz}$ up to $18 \mathrm{GHz}$. A major achievement is the study of millimeter wave beamforming designs that operate from 22-80 GHz, fabricated using low-cost printed circuit board (PCB) methods. This low-cost fabrication approach and associated testing of the beamforming arrays are unique and game-changing. 


\section{DEDICATION}

To my parents and all my supporters. 


\section{ACKNOWLEDGMENTS}

I would like to express my deepest gratitude to my advisor Prof. John L. Volakis, for his excellent mentorship across my entire pursuit of Ph.D. He has always been an energetic and dedicated model that all his students look up to. I feel privileged to be his student, having received exceptional support and guidance over the past

years.

I would like to thank my co-advisor Prof. Elias Alwan for his detailed guidance and constant patience. I'm truly grateful to have him as a mentor during my Ph.D. journey. He has helped me grow and learn since the first days of my research career, and he could not be a better friend.

I would also like to thank Prof. Shubhendu Bhardwaj for being on my dissertation committee, as well as on my candidacy and qualifying examinations committees in the past. And also Prof. Arvind Agarwal to be on final exam committee. I have been incredibly lucky to have many friends and mentors during doctoral study. I would like to thank my wonderful past and present colleagues Jingni Zhong, Ushemadzoro Chipengo, Brock DeLong, Dimitrios Siafarikas, Alexander Hovsepian, Maxence Carvalho, Matthew Nichols, Constantinos Zekios, and Nicholas Russo for their friendship and support over the years. I'd like to give special thanks to my friend and mentor Prof. Satheesh Bojja-Venkatakrishnan, whose guidance in my final year was crucial and beneficial.

Most of all, to my parents and my girlfriend Diana, for their love, support, and patience that has helped me complete this stage in my life. This would not have been possible without their help and encouragement along the way. 


\section{TABLE OF CONTENTS}

1. INTRODUCTION . . . . . . . . . . . . . . . . . 1

1.1 Applications of Ultra-Wideband Antennas and Arrays . . . . . . . . . . . 1

1.2 Introduction to Ultra-Wideband Antenna . . . . . . . . . . . . . . 3

1.3 Introduction to Ultra-Wideband Arrays . . . . . . . . . . . . . . 5

1.3.1 Ground Plane Effects . . . . . . . . . . . . . . . . . . . . . 6

1.3.2 Overview of Ultra-Wideband Phased Arrays . . . . . . . . . . . . 7

1.4 Tightly Coupled Arrays _. . . . . . . . . . . . . . . . . . . . 10

1.4.1 Fundamental Limits of TCDA . . . . . . . . . . . . . . . . . . . 11

1.4 .2 Integrated Feeds . . . . . . . . . . . . . . . . . . . . . 14

1.4.3 Resistive Loading for $>$ 9:1 Bandwidth . . . . . . . . . . . . . 16

1.4.4 Superstrates for Low Angle Scanning . . . . . . . . . . . . . . . . . . 17

1.4.5 Higher Frequency Operations . . . . . . . . . . . . . . . . . . . . . . 19

1.4 .6 Reconfigurable TCDA . . . . . . . . . . . . . . . . . . . 21

1.4.7 Platform Applications . . . . . . . . . . . . . . . . . . . . 22

1.5 Challenges in Modern UWB Aperture Design . . . . . . . . . . . . . 23

1.5.1 Achieving Larger Bandwidths in Lower Volumes . . . . . . . . . . . . . . 23

1.5.2 Differential Feeding for Integrated Radios . . . . . . . . . . . . . . 23

1.5.3 UWB Millimeter-wave Solutions . . . . . . . . . . . . . . . . . . 25

1.6 Contribution and Organization of this Dissertation . . . . . . . . 26

2. MEASUREMENT OF ULTRA-WIDEBAND STATE-OF-THE-ART TCDA 29

2.1 A Wideband Array for Small Platforms . . . . . . . . . . . . . . . . . 29

2.1.1 Design and Simulation of 9:1 TCDA . . . . . . . . . . . . . 30

2.1.2 Design Construction and Measured Results . . . . . . . . . . . . . 36

2.2 An Ultra Wideband Array for Small Platforms . . . . . . . . . . . . . . . 39

2.2 .1 Design and Simulation of 12:1 TCDA . . . . . . . . . . . . . . 39

2.2 .2 Finite Array Effects . . . . . . . . . . . . . . . . . . . . . 45

2.2.3 Design Construction and Measured Results . . . . . . . . . . . . . 47

2.3 An Extremely Wideband Array for Small Platforms . . . . . . . . . . . . 51

2.3.1 Design and Simulation of EWB TCDA . . . . . . . . . . . . . 52

2.3.2 Fabrication and Measured Results . . . . . . . . . . . . . . 61

3. UWB DIFFERENTIAL FEEDS . . . . . . . . . . . . . . 68

3.1 S-Band Differentially Fed TCDA Design . . . . . . . . . . . . 70

3.1 .1 Differential Feeding Network . . . . . . . . . . . . . . . . . 70

3.1 .2 Differentially Fed TCDA Simulations . . . . . . . . . . . . . . 73

3.2 D-TCDA Prototype Fabrication and Measurements . . . . . . . . . . 75

3.2 .1 D-TCDA Prototype Fabrication . . . . . . . . . . . . . . . . 75

3.2 .2 D-TCDA Measurements . . . . . . . . . . . . . . . . . . 77

3.3 Ku-Band Differentially Fed TCDA Design . . . . . . . . . . . 81 
3.3.1 Ku-Band Differentially Fed TCDA Simulations . . . . . . . . . . . 81

3.3 .2 Planar Feed plan . . . . . . . . . . . . . . . . . . . . 83

4. STUDY OF LOW-COST MM-WAVE TCDA . . . . . . . . . . . . 84

4.1 Novel Mm-wave Beamformer Architecture . . . . . . . . . . . . . . . 85

4.1 .1 Multi-Layer Antenna Integration . . . . . . . . . . . . . . . . 86

4.2 Egg-Crate Design with Simplified Balun Feed . . . . . . . . . . . . 87

4.2.1 Simplified Marchand Balun for $30 \mathrm{GHz}$ Operation . . . . . . . . . . . 87

4.2.2 Simplified Marchand Balun for $70 \mathrm{GHz}$ Operation . . . . . . . . . . . . 91

4.3 Differential 5G Millimeter-Wave Array . . . . . . . . . . . . . . . 96

4.3 .1 Differentially Fed TCDA Design . . . . . . . . . . . . . . . . . . 98

4.3.2 Removal of E-Plane Scan Resonance . . . . . . . . . . . . . . . . 99

4.3.3 Fabrication of Differential mm-Wave TCDA . . . . . . . . . . . . . 101

4.4 Feeding Approaches and Fabrication . . . . . . . . . . . . . . . 102

4.4 .1 Ball-Grid-Array . . . . . . . . . . . . . . . . . . . . . . . . 103

4.4 .2 UWB Balun . . . . . . . . . . . . . . . . . . . . . . . . 104

4.4 .3 Wilkinson Divider . . . . . . . . . . . . . . . . . . . 105

$4.4 .4 \quad$ Ka-Band Array Feed . . . . . . . . . . . . . . . . . . . . . . 106

4.4.5 V-Band Array Feeds . . . . . . . . . . . . . . . . . . . . . 107

4.4.6 De-embedding Process . . . . . . . . . . . . . . . . . . . . . . . 112

4.5 Link Budget Analysis and Communication Range . . . . . . . . . . . 113

5. CONCLUSIONS AND FUTURE WORK . . . . . . . . . . 115

5.1 Summary of This Work . . . . . . . . . . . . . . . . . . 115

5.2 New Work . . . . . . . . . . . . . . . . . . . . . . . . 117

5.2.1 Fabrication and Measurements of mm-wave Arrays . . . . . . . . . 117

5.2 .2 Aperture in Aperture . . . . . . . . . . . . . . . . . . . . . 118

5.2.3 Novel loading for TCDA with Extended Bandwidths and Lower Profiles119

BIBLIOGRAPHY . . . . . . . . . . . . . . . . . . . 121

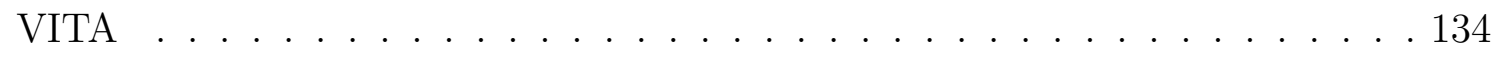




\section{LIST OF FIGURES}

FIGURE

PAGE

1.1 Modern frequency spectrum assignments from $[\mathrm{spe}] . \ldots \ldots . . . . .2$

1.2 Typical wideband antennas: (a) Planar Monopole antenna from [KKYY18]

(b) Helix antennas from [Sen55] (c) Quadruple Ridged Flared Horn antenna from [BMIM16] (d) Spiral antenna from [BF07] and (e) Bunny Ear antenna from $[$ LL93]. . . . . . . . . . . . . .

1.3 Simplified circuit model of an antenna acting as a impedance transforming transducer from a $50 \Omega$ source to (a) free space without a ground plane and (b) a complex impedance with a ground plane represented by a short circuit transmission line in shunt with free space. . . . . .

1.4 Typical wideband phased arrays: (a) Interwoven Spiral Array from [TSV11] (b) Connected-slot array from [CSN18] (c) Fragmented aperture array from $\left[\mathrm{MBL}^{+} 11\right]$ (d) Vivaldi array from [LKV18] and (e) Current Sheet array from $[$ Bal08]. . . . . . . . . . . . . . . . . . 9

1.5 (a) Coupled array over a ground plane with (b) equivalent circuit highlighting the capacitive coupling employed to cancel the ground plane inductance from $[\mathrm{ASV} 12] \ldots \ldots$. . . . . . . . . . . .

1.6 (a) The first TCDA with integrated feed from [DSV13c]. (b) The first dual polarized R-Card loaded TCDA-IB from [PV16a]. (c) The first TCDA with FSS superstrate for $70^{\circ}$ Scan from [YGV16]. (d) The first mm-wave TCDA $[\mathrm{NMV} 18] \ldots \ldots \ldots \ldots \ldots$

1.7 Maximum realizable bandwidth for an infinite-order planar array above a PEC-ground with loss-less substrate material, pointing to broadside from $[$ DSV13a]. . . . . . . . . . . . . . . . . . . . 13

1.8 (a) Pictoral representation of Marchand balun with (b) equivalent circuit. 15

1.9 (a) Magnitude (solid) and phase (dashed) of plane wave reflection coefficient of the ring-type resistive FSS, referenced to array surface. (b) VSWR of infinite array with and without a resistive FSS from $[$ MSV12a $] \ldots \ldots \ldots \ldots \ldots \ldots \ldots \ldots$

1.10 (a) Unit cell of PCB TCDA from [NMV18] with (b) representation of mm-wave Marchand balun with equivalent circuit. . . . . . . . . . . 21

1.11 Outline of designed and measured TCDA within this dissertation. . . . . 28

2.1 Pictorial representation of the designed 9:1 TCDA. . . . . . . . . . 30

2.2 Simulated Performance of UWB TCDA with (a) one overlapping pad,

(b) two overlapping pads, and (c) a VSWR comparison of the two. . 31 
2.3 Geometrical model of (a) dielectric slab with surface waves (SWs) and

(b) transmission line equivalent circuit that led to the (c) FSS superstrate that removes SWs. . . . . . . . . . . . . 33

2.4 Simulated Performance of 9:1 TCDA. . . . . . . . . . . . . . 35

2.5 Fabricated 9:1 TCDA with notch cut-outs shown for each polarization. . 37

2.6 Measured VSWR and broadside gain of center element. . . . . . . . . . 37

2.7 Measured E-Plane Pattern of 9:1 TCDA. . . . . . . . . . . . . . . 38

2.8 Measured H-Plane Pattern of 9:1 TCDA. . . . . . . . . . . . . . . . . . 38

2.9 Pictorial representation of the designed TCDA with R-Card (a) $3 \times 3$ array, (b) unit cell, and (c) feed network. . . . . . . . . . . . 40

2.10 (a) Equivalent circuit of ground plane with free space above and (b) the addition of an R-Card. The (c) input impedance of both cases show how the resistive card extends the frequency band where the ground plane resonance is a short circuit. . . . . . . . . . . . . . . . . 42

2.11 Simulated infinite array VSWR in principle planes $(\mathrm{E} / \mathrm{H})$ with scanning down to $60^{0}$ from broadside. . . . . . . . . . . . . . . . . . . 44

2.12 Simulated broadside gain of unit cell with more than $30 \mathrm{~dB}$ polarization purity. . . . . . . . . . . . . . . . 44

2.13 Finite array simulation of 12:1 TCDA showing (a) radome geometry with (b) simulated gain degradation in reference to an array surrounded by free space. . . . . . . . . . . . . . . . . . 44 4

2.14 Fabricated $7 \times 11$ array secured in anechoic chamber. . . . . . . . . . 47

2.15 Unit cell details of dual polarized D-TCDA used for infinite array simulation. Views of the identical printed metal layers are given. The metal layers are spaced equidistant by substrate layers with $\varepsilon_{r}=3.0$. 48

2.16 (Left) Measured active VSWR of array elements. (Right) Measured broadside gain of finite array with comparison to simulation . . . . .

2.17 Measured E-Plane (left) and H-Plane (right) patterns of a center element showing scan capability down to 60 degrees using the Active Element Pattern Method. . . . . . . . . . . . . . . . . . 50

2.18 Pictorial representation of the EWB TCDA with FSS R-Card (a) showing the a $4 \times 4$ section of the finite array operating from $0.13-7.63$ $\mathrm{GHz}$. The (b) $24.5 \mathrm{~mm} \times 24.5 \mathrm{~mm}$ unit cell is detailed with capacitive overlaps and (c) the tapered balun stripline feed layers. . . . . . . . . 51 
2.19 Floquet port reflection response with plane-wave excitation of TCDA unit cell without R-Card. . . . . . . . . . . . . 53

2.20 Progression of the 58:1 enabling R-card with the (a) 1-Layer [MSV13, PV16a] (b) 2-Layer (c) 4-Layer $\left[\mathrm{MBL}^{+} 11\right]$, and (d) Optimized FSS Designs (this work). . . . . . . . . . . . 5 5

2.21 Pictorial representation of (a) the novel 58:1 enabling FSS R-Card showing square loop design with (b) unit cell dimensions of the notched teeth. (c) Simulated $S_{11}$ magnitude and phase response of a plane wave reflected from an infinite ground plane in presence of the FSS R-Card .................... . . 5 55

2.22 Simulated (a) Marchand balun, (b) co-planar waveguide (CPW) to coplanar stripline (CPS) balun, and (c) tapered balun feeds investigated to excite the EWB dipoles, with (d) a comparison of insertion loss. . . . . . . . . . . . . . . . . .

2.23 (a) Equivalent TCDA circuit with FSS R-Card loading and (b) input impedance verification of the circuit model versus HFSS. . . . . . . . 58

2.24 (a) Simulated infinite array active VSWR in principle planes $(\mathrm{E} / \mathrm{H})$ with scanning down to $60^{\circ}$ from broadside. (b) Realized gain in principle planes $(\mathrm{E} / \mathrm{H})$ for simulated unit cell with scanning down to $60^{\circ}$ from broadside. (c) Realized gain in D-plane for simulated unit cell with scanning down to $60^{\circ}$ from broadside. . . . . . . . . . . . . 59

2.25 Fabricated antenna cards with bowed feeds (left) and with Styrofoam supports (right). . . . . . . . . . . . . . 60

2.26 Fabricated $12 \times 12$ array. . . . . . . . . . . . . . . 6 61

2.27 (a) Fabricated FSS Resistive Card with (b) insertion of antenna cards. . 62

2.28 Fabricated tapered balun used to excite the tightly coupled dipoles. Note that the exponential taper in the feed line (Back) is mirrored as the tapered cut on the opposite side of the board (Front), each according to $Y(z)=(D / 2) e^{-[\ln (A / D) / E]^{z}} \ldots \ldots \ldots$. . . . . . .

2.29 Measured E-Plane (left) and H-Plane (right) active VSWR of the center array element with scanning to $45^{\circ}$ and $60^{\circ}$ from broadside. . . . . . 64

2.30 Measured coupling of the center element to other array elements at various distances. . . . . . . . . . . . . . . . .

2.31 Measured broadside active VSWR of the center, off-center, and edge array elements. . . . . . . . . . . . . . . . . 
2.32 (Left)Measured broadside gain of center element vs. simulation. The co-polarized gain of several array elements is included to show finite array effects. (Right) Measured D-plane co-polarized and crosspolarized gain at theta $0^{\circ}, 45^{\circ}$, and $60^{\circ}$ cuts. A polarization purity of $17 \mathrm{~dB}$ on average is achieved out to $\theta= \pm 60^{\circ}$. . . . . . . . . 66

2.33 Measured E-plane (left) and H-plane (right) gain patterns showing scanning down to $60^{\circ}$ from broadside for $650 \mathrm{MHz}, 2 \mathrm{GHz}, 4 \mathrm{GHz}, 6 \mathrm{GHz}$, and $7 \mathrm{GHz}$ using the AEP method. . . . . . . . . . . . . . 67

3.1 Attributes of Marchand Balun and BWIT fed tightly coupled dipoles. . 70

3.2 Transmission line equivalent circuits of (a) Marchand balun with (b) Equivalent Circuit and (c) Differential Feeding Network with (d) Equivalent Circuit. (e) Verification of the BWIT equivalent circuit model for the fabricated design . . . . . . . . . . . . . . . .

3.3 Current vectors displayed on the BWIT feed for the $45^{\circ}$ case showing common-mode current cancellation. . . . . . . . . . . . . . . 72

3.4 Current vectors displayed on the BWIT feed for the $45^{\circ}$ case showing common-mode current cancellation. . . . . . . . . . . . . . . . 72

3.5 Various approaches to realize capacitive coupling in TCDAs and achieve wide bandwidths. . . . . . . . . . . . . . . . . . . . 74

3.6 Representation of the differential feed performance for each polarization using balanced S-parameters. . . . . . . . . . . . . . 75

3.7 Simulated infinite array VSWR in the principle planes $(\mathrm{E} / \mathrm{H})$ of the array at broadside and scanning to $45^{\circ}$. . . . . . . . . . . . 76

3.8 Realized Gain of the D-TCDA infinite array unit cell with $40 \mathrm{~dB}$ of polarization purity. . . . . . . . . . . . . 76

3.9 Unit cell details of the fabricated dual polarized D-TCDA. The mirrored metal layers are printed on 20 mil substrate layers with relative dialectic constant of $\varepsilon_{r}=2.2 \ldots \ldots \ldots$. . . . . . . . . 77

3.10 Fabricated $8 \times 8$ array. . . . . . . . . . . . . . . . . . 79

3.11 Measured active VSWR of an embedded center element as a function of frequency vs simulations. . . . . . . . . . . . . .

3.12 Measured broadside gain of an embedded center element as a function of frequency vs simulations. A polarization purity $>20 \mathrm{~dB}$ is achieved across most of the band . . . . . . . . . . . . . . .

3.13 Measured center element (a) E-Plane and (b) H-plane patterns vs simulations at $1 \mathrm{GHz}, 2 \mathrm{GHz}$, and $3 \mathrm{GHz}$. . . . . . . . . . . . 80 
3.14 D-plane patterns vs simulations at $1 \mathrm{GHz}, 2 \mathrm{GHz}$, and $3 \mathrm{GHz}$. . . . . . 80

3.15 Pictorial representation of (a) Ku band D-TCDA array, (b) unit cell with planar breakout feed structure, and (c) BWIT details . . . . . . 81

3.16 Design details of Ku band D-TCDA antenna cards . . . . . . . . . . . 82

3.17 (a) Active VSWR and (b) broadside gain of Ku band D-TCDA infinite array simulation. . . . . . . . . . . . . . . 82

3.18 Feed board for $18 \mathrm{GHz}$ D-TCDA. . . . . . . . . . . . . . 83

4.1 Integrated millimeter-wave transceiver concept. . . . . . . . . . . . . 86

4.2 Pictorial representation of Ka-band array, unit cell, and feed details. . . 88

4.3 Simulated active VSWR of Ka-band TCDA infinite array unit cell . . . 88

4.4 Realized gain in the principle planes $(\mathrm{E} / \mathrm{H})$ (left) and D-plane (right) for the simulated unit cell with scanning down to $60^{\circ}$ from broadside. 89

4.5 Realized gain patterns of Ka-band TCDA using AEP (left) and finite array simulations (right) with scanning to $\theta=0^{\circ}, 45^{\circ}$, and $60^{\circ}$. . . 89

4.6 Fabrication details for egg-crate Ka-band TCDA. . . . . . . . . . . . . . 90

4.7 Pictorial representation of egg-crate Ka-band TCDA installment and array visualization ..................... 91

4.8 Simulated active VSWR of V-band TCDA infinite array unit cell . . . . 91

4.9 Simulated active VSWR of V-band TCDA infinite array unit cell . . . . 92

4.10 Realized gain in the principle planes $(\mathrm{E} / \mathrm{H})$ (left) and D-plane (right) for the simulated unit cell with scanning down to $60^{\circ}$ from broadside. 92

4.11 Realized gain patterns of V-band TCDA using AEP (left) and finite simulations (right) when scanning to $\theta=0^{\circ}, 45^{\circ}$, and $60^{\circ} . \ldots 93$

4.12 Fabricated egg-crate mm-wave TCDA . . . . . . . . . . . . . . 94

4.13 Fabrication details for egg-crate mm-wave TCDA. . . . . . . . . . . 95

4.14 Fabricated egg-crate mm-wave TCDA with size referenced to a US quarter 95

4.15 Pictorial representation of a Dual polarized Differential TCDA array with Unit Cell of the design. . . . . . . . . . . . . . . . 97 
4.16 Magnitude of the Electric Field $E$ and Electric Current $J$ vectors in the E-Plane for $45^{\circ}$ scanning. It shows that the differential feed lines radiate (left) and the removal of common-mode resonance with the H-Wall Structure (right). . . . . . . . . . . . . . . 98

4.17 VSWR of D-TCDA using infinite array simulation (left) without H-Wall and (right) with H-Wall. . . . . . . . . . . . . . . . . . . . 99

4.18 Realized gain in the principle planes $(\mathrm{E} / \mathrm{H})$ (left) and D-plane (right) for the simulated unit cell with scanning down to $60^{\circ}$ from broadside. 99

4.19 Realized gain patterns of D-TCDA using AEP (left) and finite simulations (right) with scanning to $\theta=0^{\circ}, 45^{\circ}$, and $60^{\circ}$. . . . . . 100

4.20 Example $10 \times 10$ fabricated prototype next to a U.S. Quarter. . . . . . . 101

4.21 Stackup showing metal layers and via placement for PCB based differential TCDA. . . . . . . . . . . . . . . . 102

4.22 Effect of BGA interconnect on differential TCDA. . . . . . . . . . . . . 103

4.23 Effect of BGA interconnect on Ka-band TCDA. . . . . . . . . . . . . . . 103

4.24 Effect of BGA interconnect on V-band TCDA. . . . . . . . . . . . . . . 104

4.25 Planar UWB Balun (left) with simulated performance (right). . . . . . . 104

4.26 $28 \mathrm{GHz}$ power combiner for Balun-fed array (left). $28 \mathrm{GHz}$ power combiner for with integrated baluns for differential array (right). . . . . . 105

4.27 Signal Path of Ka-Band feed with $2.4 \mathrm{~mm}$ connector. . . . . . . . . . . . 106

4.28 Ka-Band feed with $2.4 \mathrm{~mm}$ connector. . . . . . . . . . . . . . . . 106

4.29 Simulated performance (bottom) of the balun-fed (left) and differential (right) V-band Split-band array feeds. The $2.4 \mathrm{~mm}$ feed for the low-band (top) and the ground-signal-ground feed for the high-band (middle) are also included. . . . . . . . . . . . . . . . 108

$4.3050 \mathrm{GHz}$ split diplexer circuit model. . . . . . . . . . . . . . . . 109

4.31 Electric field patterns for the diplexer at $20 \mathrm{GHz}$ (left), $50 \mathrm{GHz}$ (middle), and $80 \mathrm{GHz}$ (right). . . . . . . . . . . . . . . 110

4.32 Simulated S-parameters for the $50 \mathrm{GHz}$ split diplexer. . . . . . . . . . . 110

4.33 UWB feed structure for V-band balun-fed TCDA with $1.0 \mathrm{~mm}$ connector.111

4.34 UWB balun feed structure for V-band differential TCDA with $1.0 \mathrm{~mm}$ connector. . . . . . . . . . . . . . . . 111 
4.35 Back-to-back test piece de-embedding. . . . . . . . . . . . . . . . . . 112

4.36 Test structures for de-embedding the losses from the antenna array feeds.112

4.37 Link budget measurement using two $28 \mathrm{GHz}$ reference horns. . . . . . . 114

5.1 Pictorial representation of the fabricated differential TCDA array including an enlarged view of the unit cell with integrated feed board. 118

5.2 Pictorial representation of the Aperture in Aperture concept. . . . . . . 119

5.3 Pictorial representation novel loading techniques for TCDA using (a) magnetic loading, (b) EBG ground planes, and (c) metal FSS substrates. (d) A comparison of these methods versus the designs shown here is given . . . . . . . . . . . . . . . 120 


\section{CHAPTER 1 \\ INTRODUCTION}

\subsection{Applications of Ultra-Wideband Antennas and Arrays}

In the modern world, there is a need for wireless systems. Every cellular phone, Global Positioning System (GPS), smart watch, or any other wireless/Wifi/Bluetooth device utilizes a communication system, or transceiver, at radio frequencies (RF), to transfer data for social, personal, and business needs. A wireless system consists of an RF antenna, an analog RF front-end, and a digital back-end. Such a system has a myriad of applications, serving as remote sensing, radar, and secure communications systems. For implementation in daily life, RF transceivers are required to be reduced SWaP (Space Wight and Power) systems. Significant progress in material science and microelectronic chip technology has allowed for the miniaturization of digital systems over the past decades. However, the miniaturization of RF front-end components and antennas continue to be a significant challenge. In recent years, substantial research has been conducted into reduced size RF electronics through RF integrated circuit (RFIC) and Monolithic Microwave Integrated Circuit (MMIC) technologies. Yet, the antenna size-performance relationship is restricted by the small antenna limit, as Wheeler [Whe47] noted. To balance the trade-offs of radiation resistance, efficiency, and bandwidth, there must be a maximum usage of available volume for radiation.

The most common applications for RF transceivers are within the emerging small mobile platforms that include CubeSats, unmanned aerial vehicles (UAVs), and self-driving cars. These platforms are of great interest to scientific, military, and commercial sectors with programs involving remote sensing, radar surveillance, defense and targeting, and integration into the Internet of Things (IoT). For the 
example of space-borne CubeSats, there are two main antenna distinctions: 1) those that provide complete coverage of the visible earth and 2) those with high directive gain for up-link and down-link [Vol07]. The latter distinction of antennas, with high directive gain, have been increasingly replaced by phased arrays.

Of course, these CubeSats and unmanned vehicles need to be small and lightweight to maximize their deployment time. Concurrently, the operational frequencies for each niche application of the systems are separated by large bands in the frequency spectrum, as depicted in Fig. 1.1. This implies multiple large narrowband antennas and arrays would be needed, with tremendous SWaP demands.

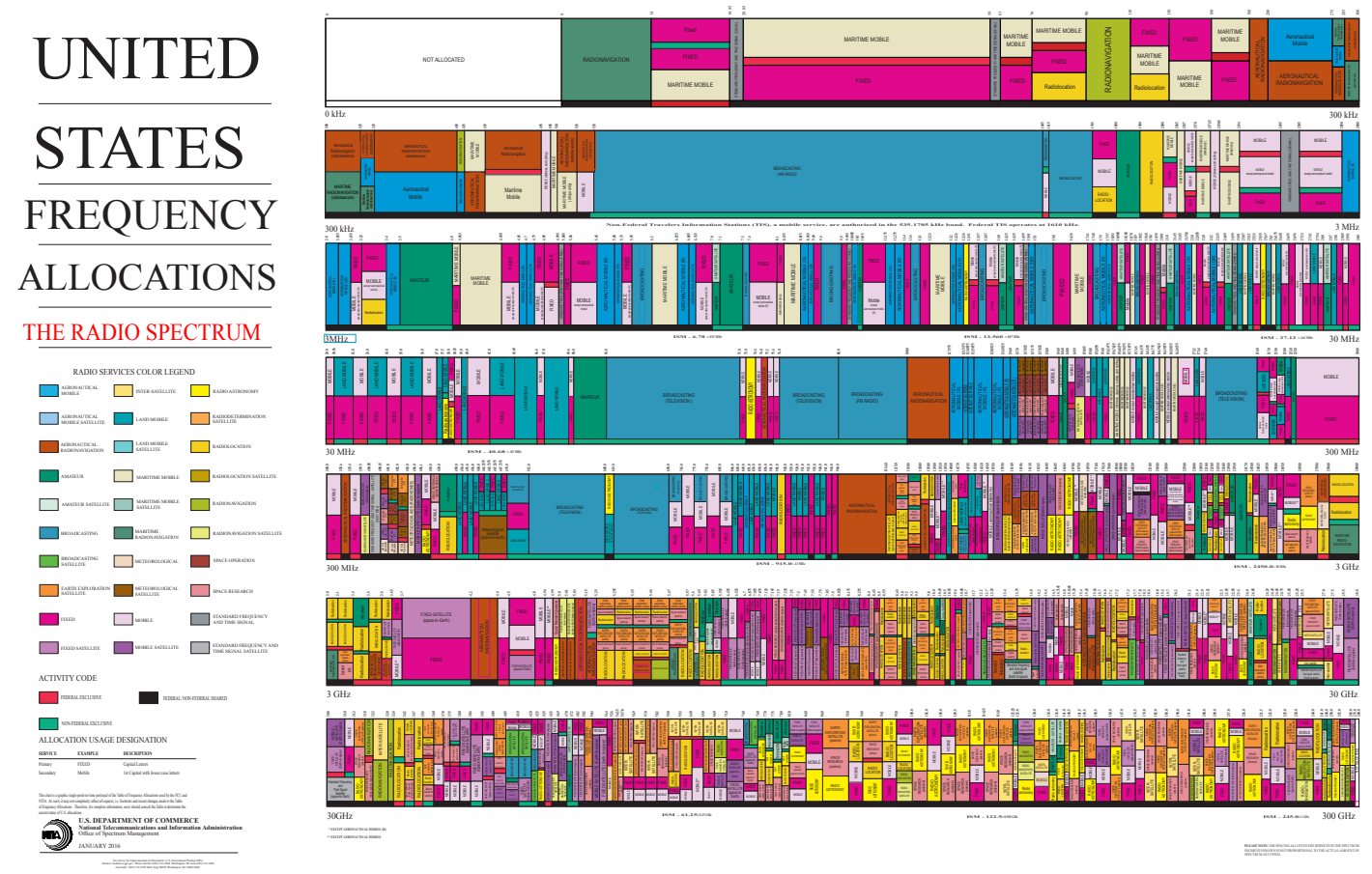

Figure 1.1: Modern frequency spectrum assignments from [spe].

To overcome this issue, ultra-wideband (UWB) antennas and arrays are brought forward to seamlessly replace multiple narrowband apertures. These UWB arrays offer orders of magnitude reduction in power, cost, and space for these systems. Even more, UWB apertures enable advanced remote sensing practices, including 
foliage penetration (FOPEN) [XN01], through-wall imaging [LN10], medical imaging [SL17], and high resolution radar [Tay94]. Similarly, Electronic Warfare (EW) [HDGV99] and radio astronomy $\left[\mathrm{dRG}^{+} 11\right]$ require large spans of bandwidth to implement secure communication's [SAV18] spread spectrum techniques such as direct sequence spread spectrum and frequency hopping.

\subsection{Introduction to Ultra-Wideband Antenna}

Antennas and RF systems are considered broadband or wideband when they can operate over a large spectrum of frequencies. The notation for this broadband is expressed as the following percentage:

$$
B=\left(f_{H}-f_{L}\right) / f_{c} \times 100 \%
$$

where $f_{H}$ and $f_{L}$ are the higher and lower cutoffs of operational frequency of a specified performance requirement, (e.g. realized gain metric or minimum reflection coefficient) and $f_{c}$ is the center frequency. This definition of bandwidth is known as the fractional bandwidth. For ratios of $>100 \%$ the bandwidth can also be defined as:

$$
B=f_{H} / f_{L}
$$

This definition, referred to as a ratio bandwidth, will be the bandwidth notation used in this dissertation. A Federal Communications Commission (FCC) review in the early 2000's defined the term UWB as having fractional bandwidth of $20 \%$ of center frequency, or $500 \mathrm{MHz}$ of capacity [Rad07]. However, in practice, this term is used for antennas that exceed a 2:1 bandwidth ratio using Eq. 1.2. There are well known approaches of designing an UWB antenna, however size and realized gain 
are always in opposition, with the goal to have both a small size and large realized gain [Whe47, Chu48]. The realized gain is defined as

$$
G=e\left(1-|\Gamma|^{2}\right) D
$$

Where $e, \Gamma$, and $D$ represent antenna efficiency, input reflection coefficient (or return loss), and directivity, respectively. The first class of UWB antennas are those that reduce the quality factor (Q) of natural resonators for increased bandwidth. For the case of a conical monopole, the introduction of multiple incoherent resonances has been shown to achieve $>9: 1$ bandwidth [ZPCV12]. The next class is the logical extension of this, where a multi-resonant structure is designed with closely spaced narrowband elements. Examples of this would be stacked patches with up to 3:1 bandwidth [SMM15].

The third class is more interesting, where diffractions on the antenna structures can be controlled or altered for UWB behavior. Examples of this antenna geometry would include the common horn antenna, known for $>10: 1$ bandwidths [BMIM16]. The other common method to reach a wideband radiation behavior is to add resistive loading, to lower the reflected power at the ports and thereby extend the impedance bandwidth. Of course, this leaves the final classification of UWB antennas to be that which are frequency scaled or frequency independent. The Log Periodic Dipole Antenna (LPDA) [Isb60] and helix antennas [Sen55] are two such examples of these antennas, which resonate at a regular rate of physical scaling.

While considering many of these aforementioned antenna as UWB candidates for airborne systems, they are inherently large and not fabricated on low cost printed circuit board (PCB). However, much work has already been devoted to mass-producing printed arrays, with planar monopoles having 7:1 bandwidths [KKYY18], two dimensional horns known as flared notch or bunny ear antennas [LL93] with $>30: 1$ 
bandwidths, and planar spirals with $>6: 1$ bandwidths [BF07]. Fig. 1.2 provides examples of the aforementioned UWB antennas. However, for many applications, a single antenna is not sufficient to meet system requirements (i.e. beamforming).

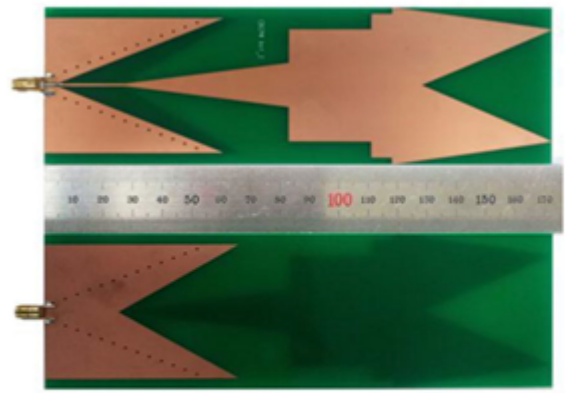

(a)

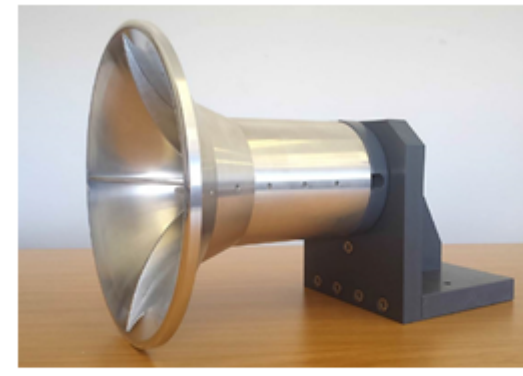

(c)

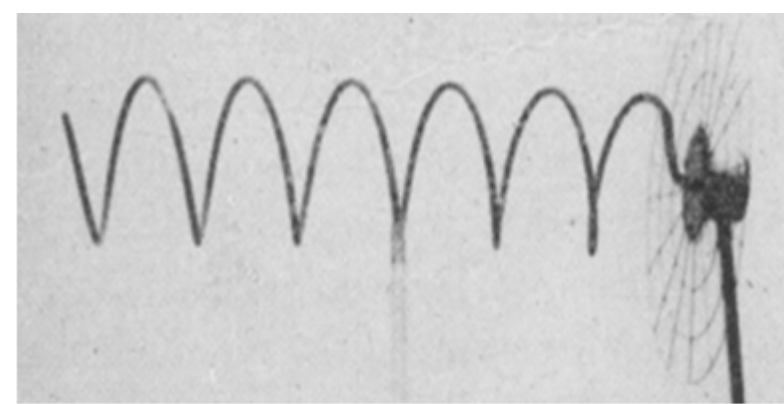

(b)

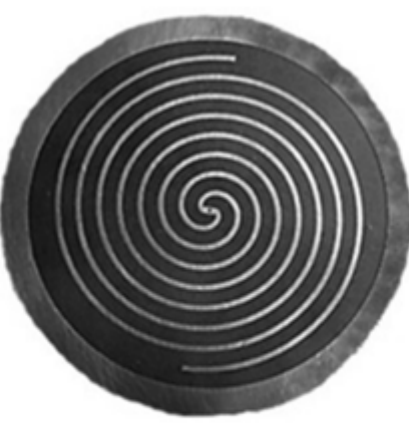

(d)

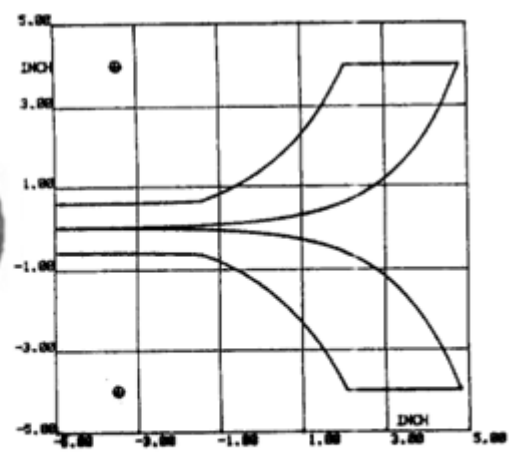

(e)

Figure 1.2: Typical wideband antennas: (a) Planar Monopole antenna from [KKYY18] (b) Helix antennas from [Sen55] (c) Quadruple Ridged Flared Horn antenna from [BMIM16] (d) Spiral antenna from [BF07] and (e) Bunny Ear antenna from [LL93].

\subsection{Introduction to Ultra-Wideband Arrays}

The need for wideband antenna systems is driven by the simultaneity of an everexpanding need for bandwidth, continually shrinking platforms, and increasingly multi-functional systems. These qualities are observed throughout the defense, scientific, and consumer-electronic sectors, where UWB electronically scanning, or 
phased, arrays provide a highly effective solution to these modern challenges. For these systems, an ultra-wideband (UWB) array replaces several narrowband systems to reduce power, cost, and save space by leveraging time, frequency, and spatial multiplexing. Such small size wideband arrays also enable increased data rates, secure spread spectrum communications, Multiple-Input-Multiple-Output (MIMO), and other vehicle to vehicle (i.e. satellite) communications. One such example is the Advanced Multi-functional RF Concept (AMRFC) $\left[\mathrm{THE}^{+} 05\right]$ that takes advantage of frequency multiplexing, supporting a 1-18 GHz operating bandwidth with 2 UWB arrays. Of course, these arrays often require directive beam-steering from mobile platforms, which in turn introduces the need for a perfect electric conducting (PEC) ground plane backing.

\subsubsection{Ground Plane Effects}

In antenna design, it in important to understand the design process in the simplest of terms. For starters, one can consider an antenna as simply a transducer between RF signals and EM waves, which transforms the system impedance of $50 \Omega$ to the $377 \Omega$ impedance of free space. However, when applications require spatial scanning and are mounted in complex platforms, a conducting ground plane is often required. This introduces impedance matching and volumetric limitations. In circuit form, while considering a periodic unit cell in an infinite array setup, this ground plane can be represented by a short circuited transmission line of finite length. This shorted shunt line provides zeros into the input impedance of the antenna at periodic frequencies where the ground plane shorts the antenna. Hence, we can point to the Wheeler and Chu relations [Whe47, Chu48] that state the inverse relationship of bandwidth

and antenna-ground plane separation. For instance, for inherently bidirectional 
antennas this extends the profile of arrays to $\lambda_{x} / 4$ (where $\lambda_{x}$ is the wavelength at the frequency $f_{x}$ ) above the ground plane. Notably this allows backward-radiated energy phase to propagate with a $180^{\circ}$ phase delay, compounded by the $180^{\circ}$ phase inversion of the perfect electric conducting ground plane which results in a coherent and constructive forward returned wave at $f_{x}$. Of course, this is a narrowband assumption, since the lambda of wideband arrays can regularly vary by more than a factor of 2 over the bandwidth, causing destructive interference at other bands. Therefore, in classical cases, physically large antennas with progressive points of radiation tend to have larger bandwidths, since the rate of change of the reactive impedance is slowed by the increased separation from the ground plane. This is illustrated in Fig. 1.3.

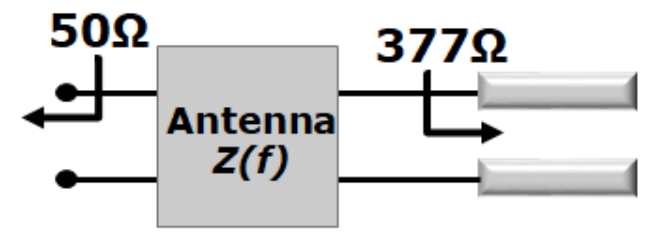

(a)

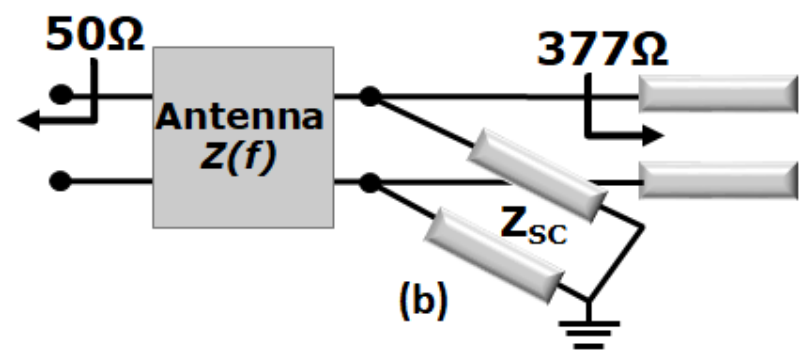

Figure 1.3: Simplified circuit model of an antenna acting as a impedance transforming transducer from a $50 \Omega$ source to (a) free space without a ground plane and (b) a complex impedance with a ground plane represented by a short circuit transmission line in shunt with free space.

\subsubsection{Overview of Ultra-Wideband Phased Arrays}

In general, an array consisting of multiple wideband antenna elements is itself wideband. For instance, stacked patch arrays have shown 2.5:1 bandwidths with $60^{\circ}$ scanning $\left[\mathrm{NEA}^{+} 11\right]$. Further, arrays of spirals have shown 10:1 bandwidths with interwoven elements [TSV11]. In recent years, with improvements in computa- 
tional ability and the introduction of fast computational electromagnetics, complex dynamic structures have been developed. These pixelated or fragmented aperture arrays have shown up to $33: 1$ bandwidths through resistive loading, with $50 \%$ efficiency $\left[\mathrm{MBL}^{+} 11\right]$.

However, the most commonly understood and accepted UWB antenna arrays were the Tapered Slot Arrays (TSA), such as the short and long Vivaldi Arrays. These arrays are known for their large operational bandwidth, viz. 10:1 impedance bandwidth, but are multiple wavelengths tall and have limited scanning capability. Thinner versions of these have recently been proposed, but at the expense of bandwidth [NGKS15]. Another variation, known as Balanced Antipodal Vivaldi Antenna (BAVA), exhibits compact profiles as low as $\lambda_{\text {High }} / 2$ with a decade bandwidth by applying capacitive coupling between elements. However, these are limited to $45^{\circ}$ scanning [EM11]. Further, a Vivaldi variation known as the Sliced Notch Antenna (SNA) array corrects the imbalanced current potentials on the Vivaldi fin edges shown to cause high cross-polarized gain [LKV18].

As an alternative to Vivaldi, connected and coupled arrays have been considered since the early 2000s. A planar wideband connected slot array was recently presented in [CSN18], where a 6-15 GHz design was shown to scan down to $60^{\circ}$ in the H-plane and $80^{\circ}$ in the E-plane. Unlike the end-fire layout of tapered slot elements, connected and coupled arrays are inherently planar, a fact which can be leveraged to produce extremely low profiles above the ground plane. This approach was inspired by the treatment of a phased array of dipoles as a continuous current sheet by Wheeler [Whe64]. Fig. 1.4 provides examples of the aforementioned UWB arrays. Since then, additional developments have improved upon Munks first 4:1 implementation of the CSA concept [Mun03, MTD ${ }^{+}$03], enabling greater bandwidth with capacitive coupling. 


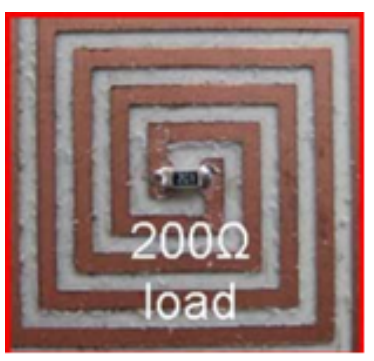

(a)

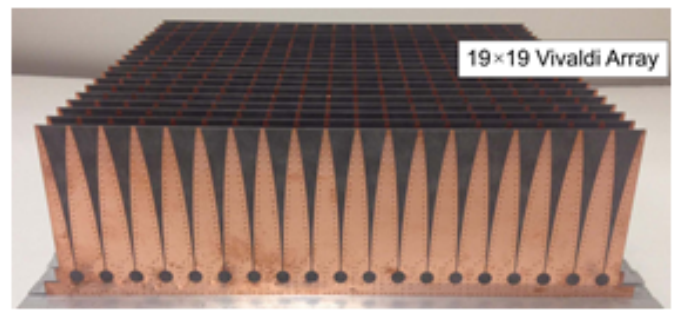

(d)

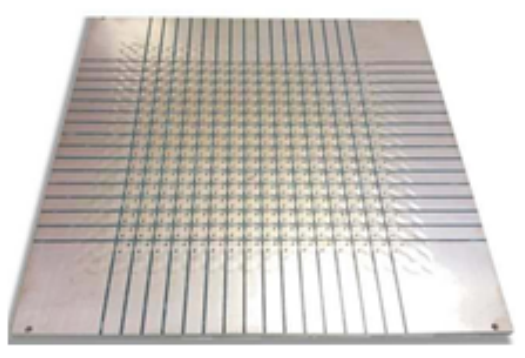

(b)

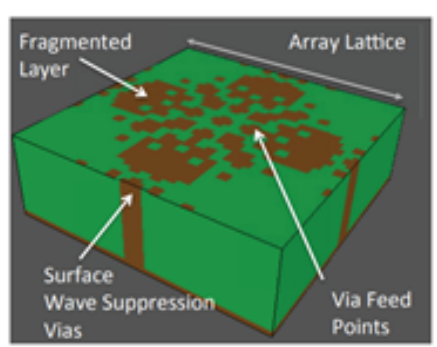

(c)

Figure 1.4: Typical wideband phased arrays: (a) Interwoven Spiral Array from [TSV11] (b) Connected-slot array from [CSN18] (c) Fragmented aperture array from $\left[\mathrm{MBL}^{+} 11\right]$ (d) Vivaldi array from [LKV18] and (e) Current Sheet array from [Bal08].

The relative benefit of coupled arrays over connected arrays stems from the ability of the capacitive coupling to partially counteract the reactance of the ground plane. This is easily understood by considering a circuit model representation of the element above the ground plane in Fig. 1.5. This model is approximate, and assumes an infinite array of linearly polarized radiators at broadside, however scanning models are presented in [RJB14]. The work in [CSN17] expands the equivalence to finite arrays using the Greens functions of tightly coupled dipoles and slots. The analysis showed that although capacitively coupled dipoles have excellent bandwidth in comparison to slots, edge effects do compromise the outer elements of finite arrays. Some variations of connected arrays have used Ring-dipole elements using lumped capacitors to emulate the capacitive coupling of tightly coupled arrays [ZB11, ZB18]. Others have discussed the areas of coupled arrays $\left[\mathrm{ZZG}^{+} 18\right]$ but the following section 
will give a detailed understanding of the Tightly Coupled Dipole Antenna (TCDA) arrays which have demonstrated the greatest impedance bandwidths and scanning performance in a low profile of $\left(<\lambda_{\text {High }} / 2\right)$.

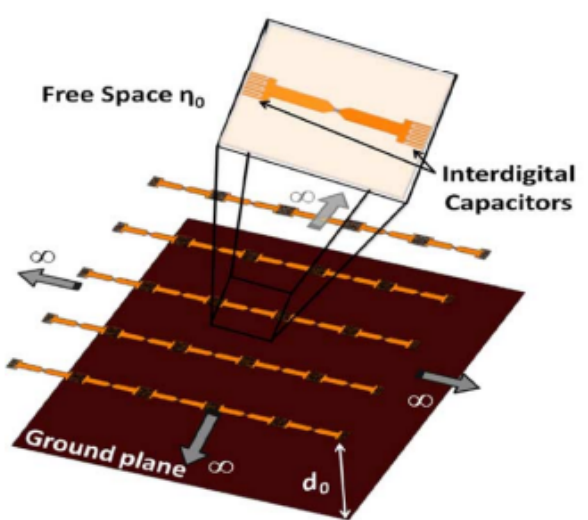

(a)

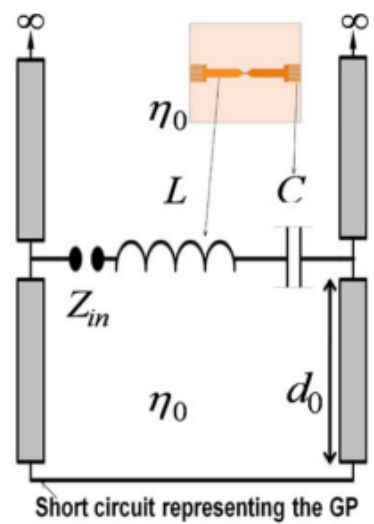

(b)

Figure 1.5: (a) Coupled array over a ground plane with (b) equivalent circuit highlighting the capacitive coupling employed to cancel the ground plane inductance from [ASV12].

\subsection{Tightly Coupled Arrays}

Following the aforementioned CSA concept, 10:1 and 4.5:1 impedance bandwidths were achieved with spiral [TSV11] and dipole elements [KCV13]. The spiral array was very low profile $\left(<\lambda_{\text {Low }} / 24\right)$, however its scanning performance was limited by scan resonances. Other attempts at coupled dipole arrays achieved up to 7.24:1 simulated bandwidths [Vog10] using square-type elements in a tightly coupled foursquare array topology [Nea99]. However, these arrays were never measured with realistic feeds. These tightly coupled arrays were greatly improved using tightly coupled dipole elements, with further bandwidth extension via optimized feeds within the array [DSV13c]. 
Such tightly coupled dipole arrays (TCDA) have been demonstrated to extend bandwidths, reduce size by more than half, and cut weight by a factor of 5 , all with a $10 \times$ cost reduction. Notably, the first use of the name TCDA was presented in [TSV13], along with the introduction of overlapping dipoles for improved bandwidth. Previous optimization of the TCDA has shown increased impedance bandwidths of 21:1 by substrate loading, scanning down to $75^{\circ}$ through FSS superstrates, reconfigurable bandwidths through internal tunable capacitors, and increased frequency operations. As a result, TCDAs have been designed and measured from $300 \mathrm{MHz}$ up to $71 \mathrm{GHz}$ with VSWR $<3$ as detailed in [Vol07]. The standout TCDA to date are found in Fig. 1.6.

\subsubsection{Fundamental Limits of TCDA}

\section{Bandwidth Limitations}

There are a few limiting factors involved in evaluating the optimal array performance of any given dipole array. Namely, the distance of the array elements from the ground plane plays a significant role in the performance of the array. The maximal bandwidth limit $(B \infty)$ for a given array thickness and material properties above a PEC-ground plane was defined in [DSV13a, DSV12, DSV14]. As seen in Fig. 1.7, the optimum bandwidth limit is 9:1 for lossless PEC backed arrays with non-magnetic substrates of $\epsilon_{r}=2.0$ (the lowest commercially available dielectric substrate is Rogers/duroid 5880LZ [588]). Higher order bandwidth limits have followed, using plane-wave scattering analysis [CK16]. 


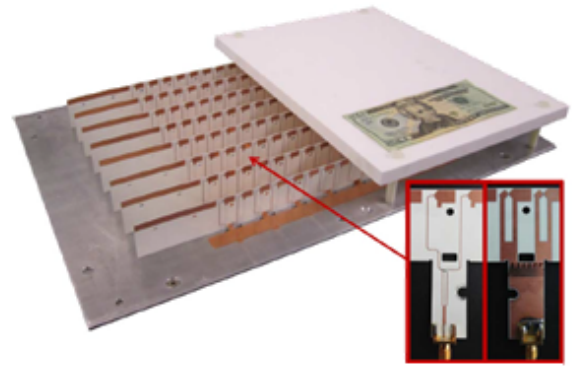

7.35:1 BW (0.6-5GHz)

UHF-L-S bands

(a)

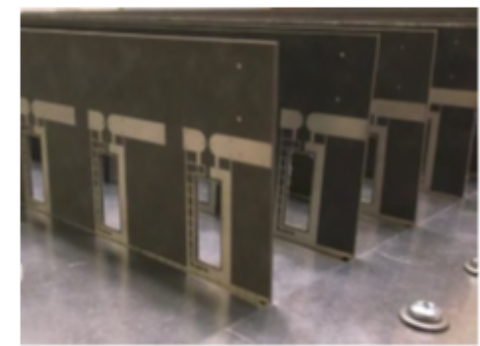

$$
\begin{gathered}
\text { 6:1 BW }(0.5-3 \mathrm{GHz}) \\
\text { UHF-L-S bands }
\end{gathered}
$$

(c)

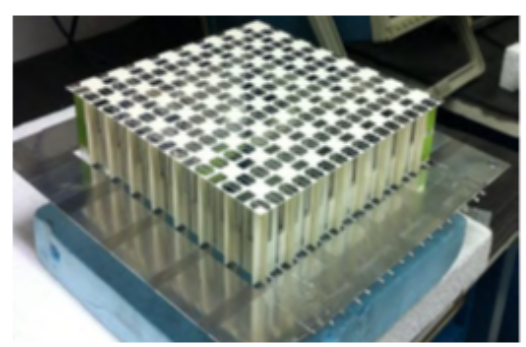

13.3:1 BW (0.29-3.9GHz) UHF-L-S bands

(b)

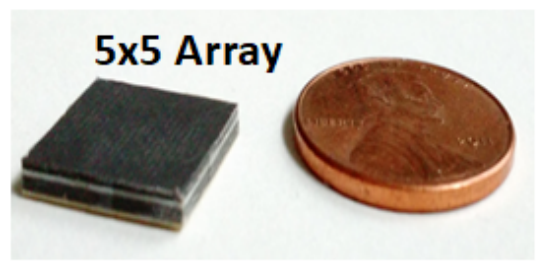

$$
\begin{gathered}
\text { 3:1 BW }(24-71 \mathrm{GHz}) \\
\text { K-Ka-V bands }
\end{gathered}
$$

(d)

Figure 1.6: (a) The first TCDA with integrated feed from [DSV13c]. (b) The first dual polarized R-Card loaded TCDA-IB from [PV16a]. (c) The first TCDA with FSS superstrate for $70^{\circ}$ Scan from [YGV16]. (d) The first mm-wave TCDA [NMV18]. 


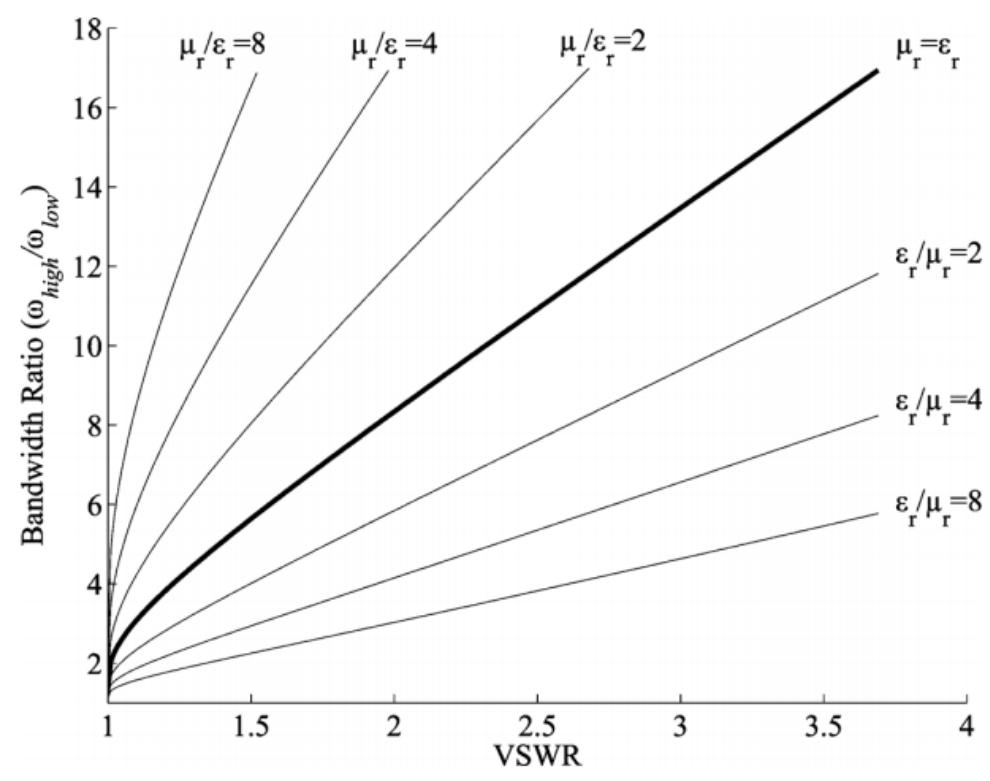

Figure 1.7: Maximum realizable bandwidth for an infinite-order planar array above a PEC-ground with loss-less substrate material, pointing to broadside from [DSV13a].

\section{Finite Array Effects}

It is common to model these tightly coupled arrays using the infinite array approach with periodic boundary conditions for fast computational analysis. Thus, by modeling only a single unit cell one can tune the performance of a large array to operate as desired. However, full-wave infinite array simulations do not account for finite array effects that include edge diffractions and diminished mutual coupling at the array edges. In addition, finite array effects usually result in a narrower operational bandwidth and mismatched peripheral array elements. Therefore, finite array simulations are required to predict more realistic array gain and efficiency. Several techniques have been investigated to improve the total array efficiency in presence of edge elements by terminating in matched or reactive loads [Tza11]. Specifically, a feeding approach using short-circuit terminated periphery elements was reported to provide up to $3 \mathrm{~dB}$ more gain and $50 \%$ higher efficiency than typical resistive 
termination for medium sized arrays [TSV13]. In addition, an improved aperture excitation for finite tightly coupled arrays was developed to improve the broadband matching of edge elements [TSV12]. Further, one excitation method based on the decomposition of time reversal operator technique (DORT) was used to maximize the array efficiency and reduce sidelobes of a finite tightly coupled array while beam scanning [SC16]. However, this is the first paper to address the finite effects of mounting on a typical airborne platform over a wideband.

\subsubsection{Integrated Feeds}

\section{Integrated Balun}

A classic problem with antenna excitation is the undesired radiation and asymmetrical pattern as a result of unbalanced feeding (i.e. common-mode) currents. The first modern TCDA were focused mainly on the capacitively coupled elements only, and used external lumped baluns [TSV13] that were found to be bulky, expensive, and limit the bandwidth of the radiators. For most modern TCDA, folded Marchand baluns printed on PCB are employed. Marchand baluns are known for their UWB performance, acting as a higher-order matching network and an impedance transformer from a standard $50 \Omega$ system to the TCDA dipole impedance of $188 \Omega$. In [DSV13c], a compact folded Marchand balun was integrated in the same PCB with a TCDA for the very first time, offering a low-cost implementation with superior scanning and impedance matching performance. A typical equivalent circuit of the Marchand balun in Fig. 1.8 (a) is depicted in Fig. 1.8. As depicted, the balun employs a series open stub $\left(Z_{o c}\right)$ and a parallel short stub $\left(Z_{s c}\right)$. The length and characteristic impedance of these stubs are tuned to achieve wideband matching. 


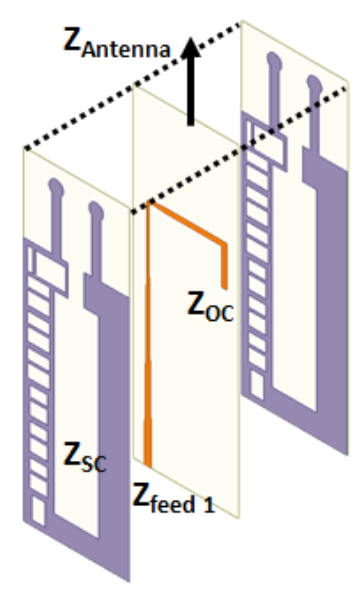

(a)

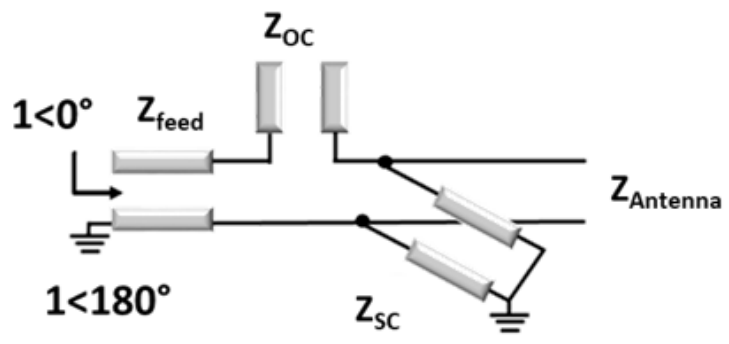

(b)

Figure 1.8: (a) Pictoral representation of Marchand balun with (b) equivalent circuit.

The first integrated baluns used a split unit cell to decrease the input resistance of the dipoles to $100 \Omega$ by splitting the periodic unit cell in half along the direction of the current density (E-plane). Then these pairs of elements were fed by a Wilkinson Power combiner to reduce the number of $\mathrm{T} / \mathrm{R}$ electronics. These power dividers were eliminated in the design presented in [YGV16] with a more effective impedance transformation using tapered cutouts in the stripline balun's ground plane. This balun has been shown achieve 14:1 bandwidth [MSV13]. In comparison external balun feeds, these TCDA-IB have proven to improve bandwidth by $30 \%$, reduce size by more than half, and cut weight by a factor of 5 , all with a $10 \times$ cost reduction. Other works have using Double-Y balun have shown only 5:1 impedance bandwidth [BG16]. To increase the bandwidth further, tapered balun have been proposed to achieve the $50 \Omega$ to $200 \Omega$ transition between the coaxial cable and the overlapped dipole terminals [BQG16], but have not yet been implemented in a low profile. 


\section{Planar Connector-less Feeds (PUMA)}

The planar ultrawideband modular array (PUMA) is a subset of the TCDA that uses a unique feed structure. The radiators of the tightly coupled dipoles operate the same as above, however the feed structure is uniquely designed to remove the need for connector, with a simple female plug connection readily available for coaxial cables or male SMP connectors. This internal balun consists of a via connecting one arm of the dipole to the signal conductor (inner conductor of the excitation coax), and the other arm is shorted to the antenna ground plane, where the outer conductor is soldered externally. An additional shorting via is employed, that can be designed to tune the common-mode currents out of band by adjusting its distance from the dipole center, as given in [HV10, Hol11]. This modular and low cost feed approach first showed moderate bandwidths with an active VSWR $<2.8$ over 7 $21 \mathrm{GHz}$ with scanning angle down to $45^{\circ}$ in $\mathrm{E}$ and $\mathrm{H}$ planes [HSV12, HV12], with recent prototypes exhibiting (6:1) bandwidth while scanning to $60^{\circ}$ [LKLV18].

\section{Optically Fed TCDA}

Due to developments in microwave photonics, optically fed TCDA have been developed $\left[\mathrm{KRS}^{+} 18, \mathrm{SBN}^{+} 15\right]$. These feeds can achieve $>3: 1$ bandwidth without the use of balun, coaxial cabling or waveguides thereby reducing the space, weight and power (SWaP) of RF systems.

\subsubsection{Resistive Loading for $>$ 9:1 Bandwidth}

To further improve bandwidth, uniform resistive sheets were used within the substrate to suppress ground plane resonances [MSV13, PV16a] over a 14:1 bandwidth. Although this approach led to increased bandwidth, it was associated with signif- 
icant gain losses over the band. While this can R-card affect radiation efficiency (about $4 \mathrm{~dB}$ of loss), it has been shown that the addition of a superstrate above the array can greatly reduce losses to only about $1.3 \mathrm{~dB}$ [MSV13].

To minimize losses and expand the bandwidth, resistive frequency selective surface have been designed. By carefully selecting the FSS resonances, this approach reduces losses to only in select bands when the distance between the ground plane the array plane is destructive. Thereby, the wave attenuation occurs only at the desired frequencies and not across the entire band. Fig. 1.9 presents the phase response of this FSS R-card and its effect on contiguous impedance matching. Rcard loading has been shown to extend the TCDA's impedance bandwidth to 21:1 [MSV12a] with an average loss of only $1.3 \mathrm{~dB}$ over the band.

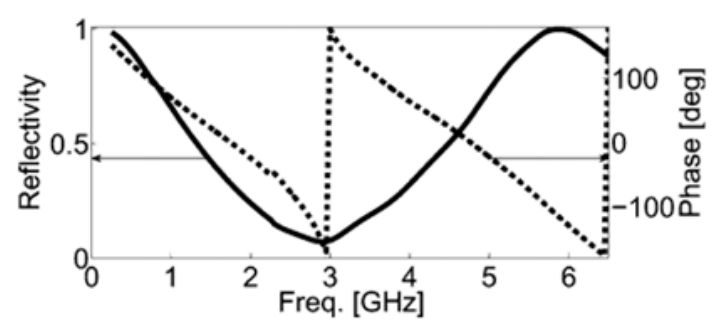

(a)

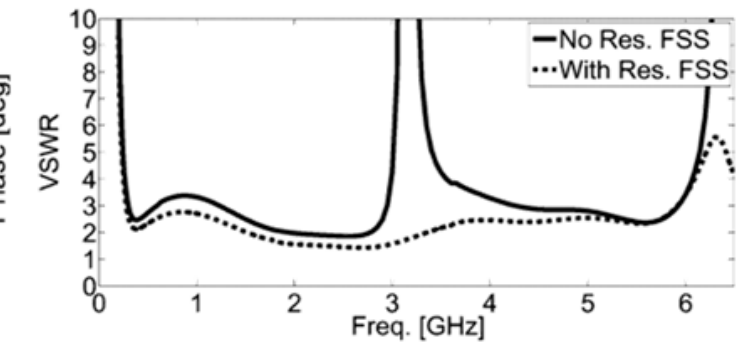

(b)

Figure 1.9: (a) Magnitude (solid) and phase (dashed) of plane wave reflection coefficient of the ring-type resistive FSS, referenced to array surface. (b) VSWR of infinite array with and without a resistive FSS from [MSV12a]

\subsubsection{Superstrates for Low Angle Scanning}

\section{Dielectric Wide-Angle Impedance Matching}

Dielectric superstrates, sometimes referred to as wide-angle impedance matching (WAIM) layers, are often found in TCDA designs to transition to the impedance of free space. Specifically, wave slowdown in the superstrate reduces the effective 
angle of incidence of free-space waves, which serves to stabilize the input impedance of the antenna across the scan volume. The dielectric superstrates are typically thin $\left(<\lambda_{\text {High }} / 4\right)$ and of low dielectric constant $\left(\epsilon_{r}<6\right)$ to avoid surface waves. As previously mentioned, superstrates are also beneficial in lossy arrays, by drawing radiation in the forward direction, away from the lossy backplane. However, a persisting issue is that the free space impedance seen by the array when scanning deviates in the two cardinal planes, following a factor of $\cos (\Theta)$ in the E-plane, or TM polarization, and $1 / \cos (\Theta)$ in the H-plane. Some authors have employed multilayer dielectric superstrates to achieve low angle scanning in both planes, at the cost of total height [WQY17, $\left.\mathrm{LGH}^{+} 16\right]$.

\section{Metasurface Wide-Angle Impedance Matching}

One alternative is the use of periodic metasurfaces, sometimes referred to as arti-

ficial dielectrics. Typically, these are sub-wavelength metallic shapes, which in aggregate produce an effective dielectric constant $\left(\epsilon_{e f f}\right)$ [Vol07]. In order to improve the scanning capabilities of the TCDA, the superstrates of bulky dielectric layer(s) were replaced with periodic printed elements to achieve wide-angle and wideband impedance matching. The approach in [YGV16] provides superior performance of 6.1:1 bandwidth $(0.5-3.1 \mathrm{GHz})$ with VSWR $<3.2$ when scanning $75^{\circ}$ in E-plane, $70^{\circ}$ in D-plane and $60^{\circ}$ in H-plane. This TCDA was the first low profile design to achieve more than 6:1 bandwidth with such wide scan angles without using a bulky dielectric material over the aperture.

Similar variations have now followed with a 7.3:1 bandwidth $(0.3-2.2 \mathrm{GHz})$ $\left[\mathrm{ZYC}^{+} 18\right]$ and scanning down to $\pm 70^{\circ}$ in E-/D-plane and $\pm 50^{\circ}$ in H-plane $\left[\mathrm{ZYX}^{+} 19\right]$. Others have introduced planar wide angle impedance matching through a novel wideband meta-surface superstrate where $70^{\circ}$ E-plane and $55^{\circ} \mathrm{H}$-plane scans are 
achieved in a 5.5:1 (0.80-4.38) GHz bandwidth $\left[\mathrm{BQZ}^{+} 19\right]$. Past designs that employed metasurfaces without exceeding $45^{\circ}$ scan capability of simple dielectrics were not included for brevity. Notably, a common way to show the scan capability is to use the Active Element Pattern, which assumes the performance of a large array is equivalent to that of a center element multiplied by its array factor [Poz94]. This is to show the measured performance of an infinite array using a perfect loss-less digital beamformer.

\subsubsection{Higher Frequency Operations}

The growing need for high bandwidth communications, particularly to mobile users, has led to the exploration of the millimeter-wave (mm-wave) spectrum. Unfortunately, the allocated frequencies for millimeter-wave communications are widely distributed. The recently allocated 5th Generation (5G) mobile and unlicensed Industrial, Scientific, Medical (ISM) bands span nearly $50 \mathrm{GHz}$, from $24-71 \mathrm{GHz}$. Whereas many millimeter-wave antenna arrays have been developed in the literature, all of these arrays utilize simple, narrow-band elements. Given the cost and space required for these arrays, there is a pressing need for broadband millimeterwave arrays, which will allow the consolidation of many different functions into a shared aperture [Vol07]. Such arrays are presented below.

A one dimensional $1 \times 8$ element TCDA with E-plane scanning to $30^{\circ}$ was designed on a multilayer substrate with coplanar, rather than perpendicular, ground plane. The array was intended for the $28 \mathrm{GHz}$ band, with measured bandwidth showed VSWR $<2$ for 22.5-32.5 GHz [SGC19]. Another design shows simulated operation from $44-100 \mathrm{GHz}$ VSWR $<3$ for $60^{\circ}$ given the use of a specialized probe [MYG18]. Further, recent mm-wave TCDA have integrated micro-electro mechani- 
cal systems (MEMS) phase shifters are into each element for real-time beam forming, for continuous wideband beam-steering antenna over the K-Ka bands (18-40GHz) [ANHS17]. However, these mm-wave arrays must have full polarization diversity and practical feeds for future implementations.

\section{On-Wafer Millimeter-wave TCDA}

One such array was developed for large-scale transceiver chipsets. This monolithically integrated multilayer phased array architecture uses a ground plane to shield from lossy substrates for an estimated radiation efficiency $>60 \%$ for the $35-75 \mathrm{GHz}$ matched band. Further, the array has future applications in mobile applications, with a low profile typical of TCDA, and $5 \mathrm{dBi}$ realized gain in a small footprint of $7 \mathrm{~mm} \times 3 \mathrm{~mm}[\mathrm{SCNS} 18]$.

\section{PCB Millimeter-wave TCDA}

To avoid the pitfalls associated with using expensive and low yield micro-fabrication techniques such as Low Temperature Co-Fired Ceramic (LTCC) another TCDA was introduced using PCB manufacturing. This PCB mm-wave-TCDA utilizes an alternative balun design, with a folded open stub in a planar orientation and coupled metal vias as the transmission line feed elements. The array in Fig. 1.10 achieved VSWR $<2.5$ for $45^{\circ}$ scanning in both planes over a range of $26-86 \mathrm{GHz}(3.3: 1$ bandwidth) [NMV18]. 


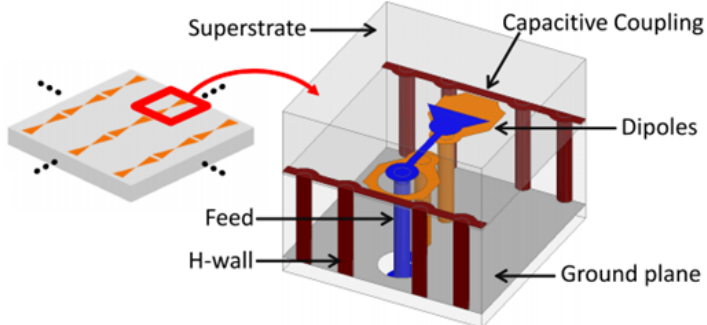

(a)
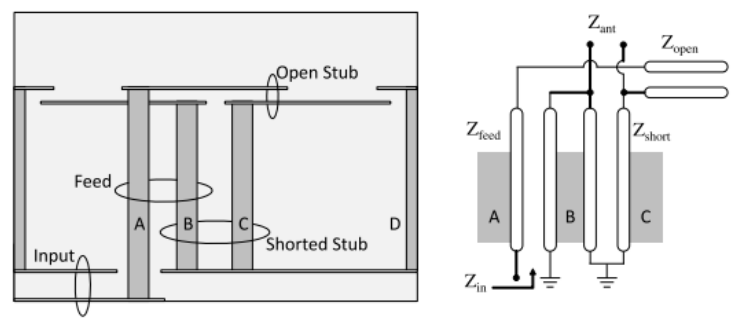

(b)

Figure 1.10: (a) Unit cell of PCB TCDA from [NMV18] with (b) representation of mm-wave Marchand balun with equivalent circuit.

\subsubsection{Reconfigurable TCDA}

\section{Tunable band reject TCDA for interference mitigation}

Recently, a 4:1 (1-4GHz) dual-polarized TCDA with tunable band-rejection capabilities was presented with scanning down to $60^{\circ}$ in all planes [PV16b]. To create a band-stop response at the aperture level, a variable capacitor was placed in shunt of the short stub of the balun to elongate the stub's electrical length and short out the dipoles. This scheme operates for all scan angles without added volume or loss, as opposed to typical tunable notch filters. A reconfigurable finite array with dynamic rejection of $>20 d B$ was demonstrated, using digitally-biased tunable capacitors. Likewise, a digitally-biased superstrate has been implemented to create a tunable bandpass filter at the antenna level [PYV16].

\section{Textile TCDA}

A novel tightly coupled dipole array (TCDA) printed on textiles achieved 30:1 bandwidth from 60-2000 MHz with realized gain ranging from 16 to $6 \mathrm{dBi}$ across the band $\left[\mathrm{ZLP}^{+} 18\right]$. The fully flexible textile-based prototype was printed on organza fabric

via automated embroidery of conductive threads (E-threads). On-body operation (e.g., placed along the arms) was measured using ground beef. 


\subsubsection{Platform Applications}

These arrays are already being implemented in military, scientific, and commercial industries. Thermal analysis was conducted on one such TCDA developed alongside NASA for Small Satellites [NMV17]. Notably, The UWB nature of these arrays were exploited for the first time in a reflect-array using a simple 3:1 TCDA [LGZ $\left.{ }^{+} 18\right]$. Even more, TCDA have already been developed for airborne radars [Tza11].

To be used to their full bandwidth potential, wideband receivers must be implemented alongside these arrays, which requires high speed ADCs. Recently, a novel on-site coding receiver (OSCR) architecture was presented to significantly reduce hardware requirement and increase instantaneous bandwidth for digital beamforming. With this, TCDA operating from $200 \mathrm{MHz}$ to $2.5 \mathrm{GHz}$ show an accurate estimate of the angle of arrival for incidence angles of $\Theta_{s}=0^{\circ}, 15^{\circ}, 30^{\circ}$, and $45^{\circ}$ away from broadside $\left[\mathrm{VPA}^{+} 17\right]$. Likewise, an 8-channel OSCR for multiple-inputmultipleoutput (MIMO) applications was shown to accurately estimate the angle of arrival with $<1.8^{\circ}$ error using two transmit signals, modulated simultaneously at $1350 \mathrm{MHz}$ and $1800 \mathrm{MHz}$ and arriving from various angles across $0^{\circ}$ to $\pm 50^{\circ}$ [VAAV17]. Moreover, element-level diplexing has also been demonstrated to enable precise aperture control across 9.5-37 GHz, covering six different radiometry and synthetic aperture radar (SAR) bands within one TCDA [ $\left.\mathrm{JSN}^{+} 18\right]$.

Further, with the growing demand for spectrum utilization, the concept of full duplex transceivers has become attractive. These simultaneous transmit and receive systems (STAR) are desired as they double bandwidth utilization. However, to realize STAR the self-interference between transmit and receive antennas must be suppressed. With the high isolation attributes of TCDA, $100 \mathrm{~dB}$ isolation was achieved for practical STAR, across a large $500 \mathrm{MHz}$ system bandwidth using polarization diversity for two user STAR communications [VAV18]. 


\subsection{Challenges in Modern UWB Aperture Design}

\subsubsection{Achieving Larger Bandwidths in Lower Volumes}

As previously mentioned, Ultra-wideband (UWB) scanning arrays enable the consolidation of multiple apertures on increasingly Size-Weight and Power and Cost (SWaP-C) constrained platforms. UWB apertures have demonstrated bandwidthgain-scanning performance such that they simultaneously support requirements for an increasing set of required antenna functions in a low profile. However, the platforms themselves are ever-shrinking, with CubeSats smaller than an apple and smart devices only few millimeters thick, all with the multi-functional requirements. This requires RF systems and antennas to be ever-shrinking without sacrificing functionality.

\subsubsection{Differential Feeding for Integrated Radios}

With the TCDA inherently being a dipole, feeding can be a limitation. Infinitely large TCDA with idealized feeds can be shown to have extremely wideband radiation characteristics, however practical feed structures may add losses and reduce bandwidth in practice. All the aforementioned TCDA employed single-ended feeds for low angle scanning over wide bandwidths. Unfortunately, these balun feeds also introduce the possibility of broadside common-mode currents, which adds design time to ensure the common-modes are tuned out of the optimized arrays.

Conversely, differential feeds inherently cancel common-mode currents at broadside, but exhibit common-modes when scanning to low angles. For instance, the UWB differential feeds in [BSC93, EO60, LLK04, dLAGGP $\left.{ }^{+} 10\right]$ were plagued by common-mode resonances when scanning in the E-plane nearing angles down to 
$45^{\circ}$. Hence, the major challenge in the design of full differential radios is the reduction of these common-mode currents present in the feed-lines when scanning. Notably, common-modes arise for particular scan angles and frequencies in the differential feed lines due to destructive mutual coupling between elements in presence of a third conductor (i.e. ground plane). If not addressed properly, these common-modes radiate in a manner that significantly reduces total efficiency and impedance bandwidths of balanced antenna structures [S. 08]. The most prevalent way to suppress these scan-dependent common-modes is to employ a balanced-to-unbalanced feed (i.e balun), thereby avoiding differential feeding altogether [DSV13c, PV16a, YGV16, NMV18, LKLV18, HV11]. However, as previously mentioned, this introduces the need to tune out broadside common-mode currents. Other methods have been explored to remove such modes by employing conductive E-Plane walls to disrupt the fields in single polarized arrays [BSC93, EO60], or by using resistive terminations to attenuate the common-modes [LLK04]-[dLAGGP $\left.{ }^{+} 10\right]$.

A key benefit of a differential array is its direct compatibility with existing wideband differential components [Spi16] that enable high dynamic range and linearity. Further, differential signals provide inherent lower distortion in response to noise and interference from power supplies. Even more, distortions due to even order harmonics from nonlinear devices can be suppressed by differential feeds [Poz12]. The frequency scalable differential feed presented here is directly compatible with differential power amplifiers, which removes the phase and amplitude mismatch and associated losses of external baluns. Therefore, an UWB low-angle scanning differentially fed array is of great interest. 


\subsubsection{UWB Millimeter-wave Solutions}

With rapidly increasing demand for high data rates, wirelessly connected devices (e.g. cell phones, cars, watches) need multi-functional radios at millimeter-wave (mm-wave) frequencies. The $5 \mathrm{G}$ boom of the past decade has begun to show real promise at the time of this dissertation, as discussed by global leaders in communications (e.g. Verizon, Qulacomm, and Ericsson). In years prior, there was much doubt in the viability of $5 \mathrm{G} \mathrm{mm}$-wave systems due to the excessive path losses, lack of supporting electronics, and limitations of legacy mm-wave systems used in space. However, recently there have been great strides in beamforming techniques, channel coding, commercialization, and deployment of $5 \mathrm{G}$ mm-wave systems. With the support and interest of the commercial sector, the FCC has opened many mmwave band allocations for use in Vehicular-to-Vehicular applications (28 GHz), ISM communication bands $(60 \mathrm{GHz})$, and vehicular radar bands $(77 \mathrm{GHz})$.

To accommodate these sparsely allocated spectrums, antennas must be ultrawideband (UWB) and integrated with transceivers. Further, the high path-losses at these frequencies require high gain arrays with wide-angle spatial coverage. At present, many of available UWB arrays are focused on narrowbands and fabricated using expensive micro-fabrication techniques such as LTCC [LLJ+19] and photolithography [SCNS18]. Recent UWB arrays have addressed the expensive fabrication problem with flip-chip printed circuit board (PCB) antenna fabrication in mind [NMV18]. However, there is still work to be done towards obtaining the polarization diversity and differential feeding necessary for 5G applications [Spi16]. Moreover, active feeding of large $(10 \times 10)$ phased arrays over UWB is a significant design challenge. 


\subsection{Contribution and Organization of this Dissertation}

The focus of this dissertation is on UWB arrays for mobile platforms. In particular, we present:

- Significant bandwidth improvements for TCDA

- Differential feeding for system integrated TCDA

- Mm-wave TCDA that leverage commercial PCB fabrication techniques to provide the unprecedented bandwidth, scan range, and efficiency for the emerging fifth generation $(5 \mathrm{G})$ millimeter wave technologies

The remainder of this dissertation is organized as described in Fig. 1.11. Chapter 2 outlines the development and measurement of three state-of-the-art UWB TCDA. The first TCDA presented herein obtains the 9:1 loss-less bandwidth limit with $60^{\circ}$ scanning. The second TCDA uses resistive loading to surpass this limit for a 12:1 bandwidth with $60^{\circ}$ scanning for the first time, and is the first TCDA to be designed with the specific needs of an airborne platform. The third TCDA introduces a ground-breaking resistive FSS and novel feed that enables a 58:1 broadside bandwidth with $60^{\circ}$ conical scanning.

Chapter 3 outlines the development and measurement of a novel TCDA with differential feed. This is the first UWB differential array to scan to low angles without resonances in the E-Plane over an unprecedented 8:1 bandwidth. The design and operation of the enabling Balanced Wideband Impedance Transformer (BWIT) feed structure is discussed.

Chapter 4 outlines the development and study of mm-wave TCDA and UWB active array feeding through vertical interconnects. The first two UWB array are focused on low cost manufacturing, with single board PCB construction to cover the 
majority of the $5 \mathrm{G}$ bands with $60^{\circ}$ scanning. The third UWB array is focused on low cost manufacturing with differential feeding. Again, a novel feed structure, denoted as an H-Wall enables $60^{\circ}$ scanning on low cost PCB. The three aforementioned mmwave arrays are designed to use a Ball-Grid-Array (BGA) of $0.2 \mathrm{~mm}$ diameter solder balls to realize the vertical interconnection of the antenna stackup to the planar feed board. Measurements for these arrays will be provided in future works.

Chapter 5 provides a summary of this work and discusses future works related to broadband antennas and arrays for mobile platform applications. 


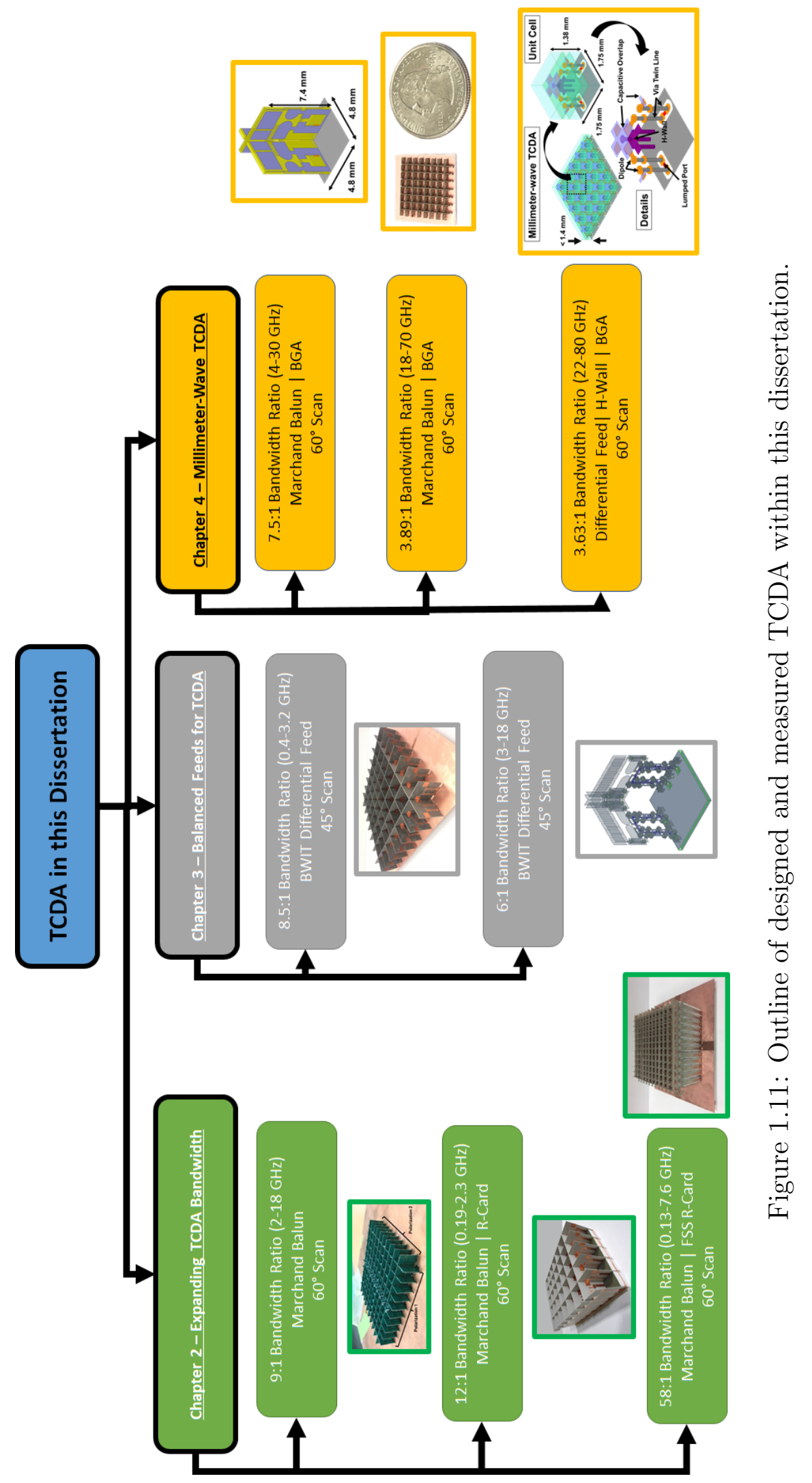




\section{CHAPTER 2}

\section{MEASUREMENT OF ULTRA-WIDEBAND STATE-OF-THE-ART TCDA}

\subsection{A Wideband Array for Small Platforms}

In this section, a dual polarized vertically printed array with 9:1 bandwidth and low-angle scanning capability is presented, as depicted in Fig. 2.1. Improving on the performance of past works [DSV13c, JR07, YGV16, NMV17], the array design presented here combines FSS superstrates for an optimum dual polarized prototype operating from $2 \mathrm{GHz}$ through $18 \mathrm{GHz}$, with scanning down to $60^{\circ}$ from broadside. Simulations are verified with the measured results of a fabricated $11 \times 11$ prototype.

This array has a multitude of applications in airborne platforms, with coverage of altimetry, weather, surveillance, and fire control radar bands. For instance, this array can seamlessly easily replace the large gimballed reflector antennas for the Defense Satellite Communication System (DSCS) that operates from $7.25 \mathrm{GHz}$ to 8.40 GHz, the $4.2 \mathrm{GHz}$ to $4.4 \mathrm{GHz}$ patch arrays that function as altimeter radars, the 9.375 GHz reflectors used for weather radar, and countless other narrowband systems [Vol07]. Also, this array is a good candidate for Electronic inelegance (ELINT) systems, that typical reside in the $2 \mathrm{GHz}$ to $18 \mathrm{GHz}$ bands.

Further, the array is of low profile and high gain, making it an ideal candidate for SATCOM communications. The NASA Space, Near-Earth, and Deep-Space Networks (SN, NEN, and DSN respectively) are also covered within this $2 \mathrm{GHz}$ to $18 \mathrm{GHz}$ bandwidth [NAS15]. The ability to employ a single source solution for communications between satellites and ground stations is essential for future reduced size platforms, such as CubeSATS. The large bandwidths provided with this array 

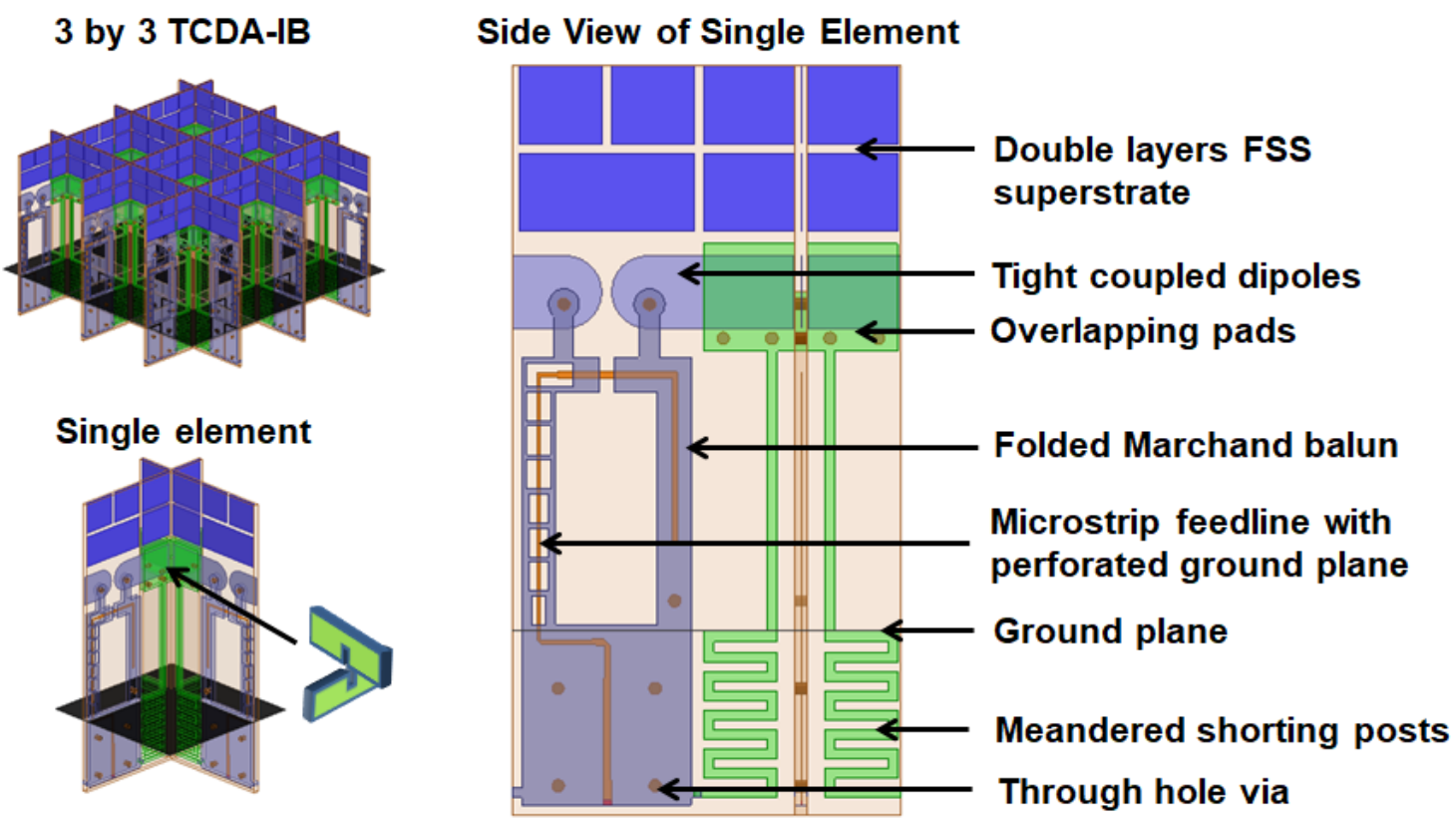

Feed

Figure 2.1: Pictorial representation of the designed 9:1 TCDA.

also result in high data throughput. Overall, this array introduces significant space, weight, and power savings for airborne and space-borne platforms.

\subsubsection{Design and Simulation of 9:1 TCDA}

As discussed in Chapter 1, earlier TCDA operated at Ku band with intentional design for satellite communications [NMV17], but were singularly linear polarized and operated only over a 6:1 bandwidth with $45^{\circ}$ scanning. The 9:1 presented dual-polarized array improves on the previous design in [YGV16] with a metal FSS superstrate for low-angle scanning, in a low profile of $\lambda_{\text {Low }} / 10$. This array introduces 1) improved bandwidth through a streamlined fabrication, 2) dual polarization with an enhanced balun feed, and 3) $60^{\circ}$ scanning with a new double FSS superstrate and novel shorting posts. 


\section{Improved Bandwidth Through Increased Capacitive Coupling}

As typical with phased arrays, the dipole elements are designed with a pitch $<$ $\lambda_{H i g h} / 2$ to avoid grating lobes. It is key to the design that the polarizations are not fed concentrically, but intersect at the ends of the dipoles. The design in [PV16a] employed overlapping planar dipoles with each linear polarization printed on opposite sides of a substrate, however this required soldering to the vertically oriented stripline balun feeds. An alternative method to save fabrication and assembly time is presented here with a stripline approach. That is, with dipoles printed on the central layer and captive overlaps printed on either side of the substrate for increased coupling. Specifically, the overlapping pads are printed on the first and third layers and are connected in parallel by vias to double the capacitive coupling.

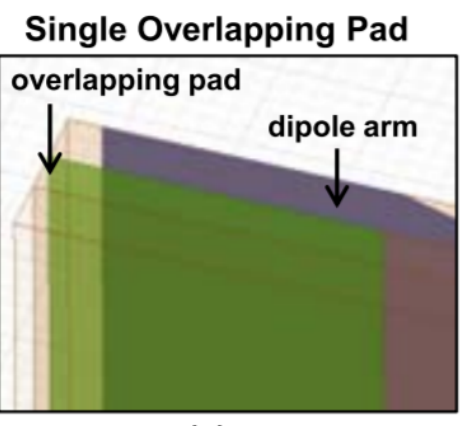

(a)

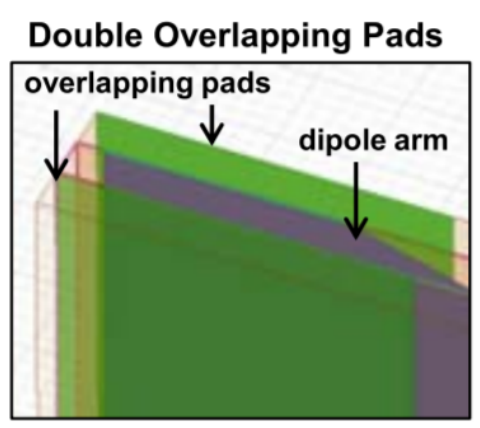

(b)

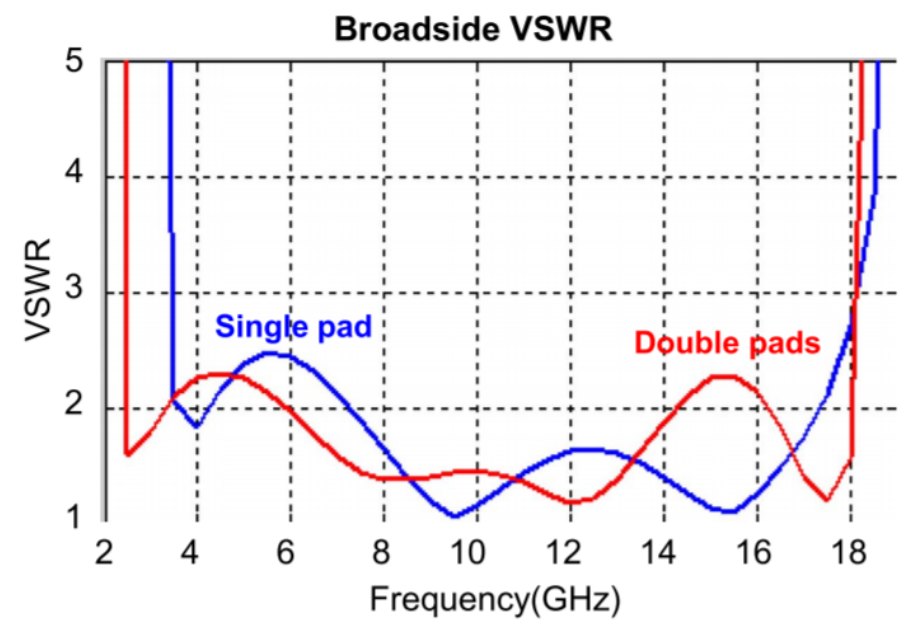

(c)

Figure 2.2: Simulated Performance of UWB TCDA with (a) one overlapping pad, (b) two overlapping pads, and (c) a VSWR comparison of the two. 
Fig. 2.2 demonstrates a VSWR comparison of the TCDA using the single and double overlapping pads. The simulated broadside VSWR is shown in Fig. 2.2(c). As shown, double overlapping pads introduce a stronger capacitive coupling among the dipole elements to effectively extend the operational bandwidth. This strong capacitance between the dipoles yields a continuous current distribution, hence this array achieves the 9:1 lossless PEC backed array limitation [DSV13b].

\section{Improved Marchand Balun}

A wideband balun is implemented to feed the UWB dipoles without introducing common-mode currents. This feed draws from [DSV13c, YGV16] with an integrated Marchand balun that acts as a higher order matching network. In practice, the Marchand balun uses a series open stub and a parallel short stub, whose impedance and lengths are tuned to achieve a broadband match. Similar to [YGV16] rectangular perforations were engraved into the shorting stubs of the balun, which act as the ground layers of the stripline feed, in order to increase the achievable characteristic impedance $\left(Z_{0}\right)$. By doing so, we remove the need for the split-cell and Wilkinson power combiner approach of works past [DSV13c, MSV13], to efficiently transfer the $50 \Omega$ port impedance to the $188 \Omega$ input impedance of the tightly coupled dipoles. The effect of these perforations on characteristic impedance is detailed in [YGV16]. However, common-mode resonances can still occur if the separation between feed lines of neighboring unit cells is large enough. To circumvent the issue, meandered shorting posts are added between the overlapping pads and the ground plane to disturb the resonance conditions. 


\section{Improved Double FSS Superstrate}

This superstrate improves on past TCDA [DSV13c, NMV17] to enable lower angle scanning, even down to $70^{\circ}$ [YGV16] while being printed on the PCB, for simple and lower cost fabrication. Past wideband array used electrically thick dielectric slabs to match the aperture to the impedance of free space. However, these superstrates limit the available scan range, as surface waves (SWs) arise when scanning to low angles. To address this issue, perforations in the dielectric layers have been proposed to reduce the effective dielectric constant and push the onset of SWs out of the intended scan range and frequency bands [LKLV18]. Here, the superstrates are periodic metallic rectangles, with large feature sizes, hence they cannot be approximated as an anisotropic dielectric material and essentially only provide capacitive loading.

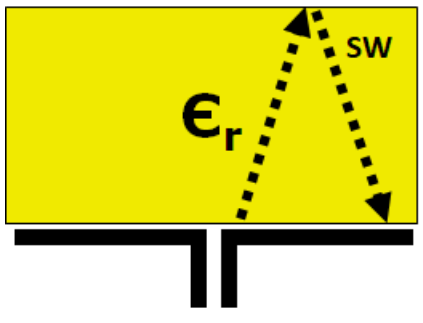

(a)

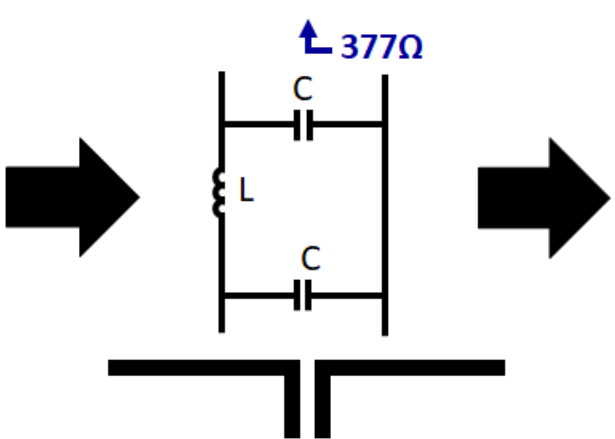

(b)

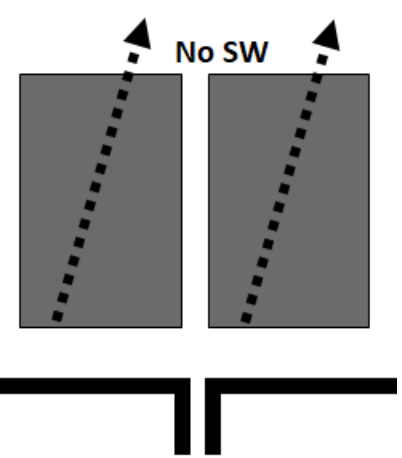

(c)

Figure 2.3: Geometrical model of (a) dielectric slab with surface waves (SWs) and (b) transmission line equivalent circuit that led to the (c) FSS superstrate that removes SWs.

Therefore, we refer the rectangular superstrate as an FSS superstrate throughout this dissertation, even though it always operates below its resonance frequency. The FSS essentially results in a shunt capacitance for a plane wave propagating along the z-axis, as seen in Fig. 2.3(b). The FSS presented here is co-optimized along with the TCDA, with larger features having greater impact on low frequency matching, and 
smaller features showing significance to the higher frequency VSWR. For our doublelayer FSS, each layer is designed to contain metallic squares of small width and height to ensure good performance at the higher end of the band. At low frequencies, since the wavelength becomes significantly longer, the closely spaced two-layer FSS rows behave such as a single FSS. That is, the smaller FSS patches are equivalently one larger FSS element at the lower frequencies and therefore accommodate the lowband performance.

\section{Simulation Results}

An infinite array simulation was used to represent the $11 \times 11$ finite element array using ANSYS HFSS v.19, with each polarization fed by a $50 \Omega$ lumped port from a microstrip-coax launcher. Our array design was initially optimized using the equivalent circuits derived in [DSV13c, MSV12b, ASV12]. These equivalent circuits model the tightly coupled dipole as a series L (dipole) and C (coupling), with the superstrate as an equivalent finite length transmission line terminated by the free space impedance of $377 \Omega$ as discussed in Chapter 1.3.2.

Notably, the plane containing the direction of the current is expressed as the E-plane, with the perpendicular plane as the H-plane, and the diagonal plane as the D-plane. The infinite array VSWR for the the E, H, and D planes, with scanning to $60^{\circ}$ is shown versus frequency in Fig. 2.4. The array provides a broadside VSWR $<3$ for $2 \mathrm{GHz}$ to $18 \mathrm{GHz}$ for an impedance bandwidth of 9:1. A reduced performance in the H-plane is expected with planar arrays, based on the variation in the Floquet mode impedance in both planes, with a $1 / \cos (\theta)$ term related to the resistance looking into the H-Plane [Whe64]. However, due to the inherently larger beamwidths at the lower frequencies, it is unnecessary scan to the low angle of $60^{\circ}$ below $5 \mathrm{GHz}$. 

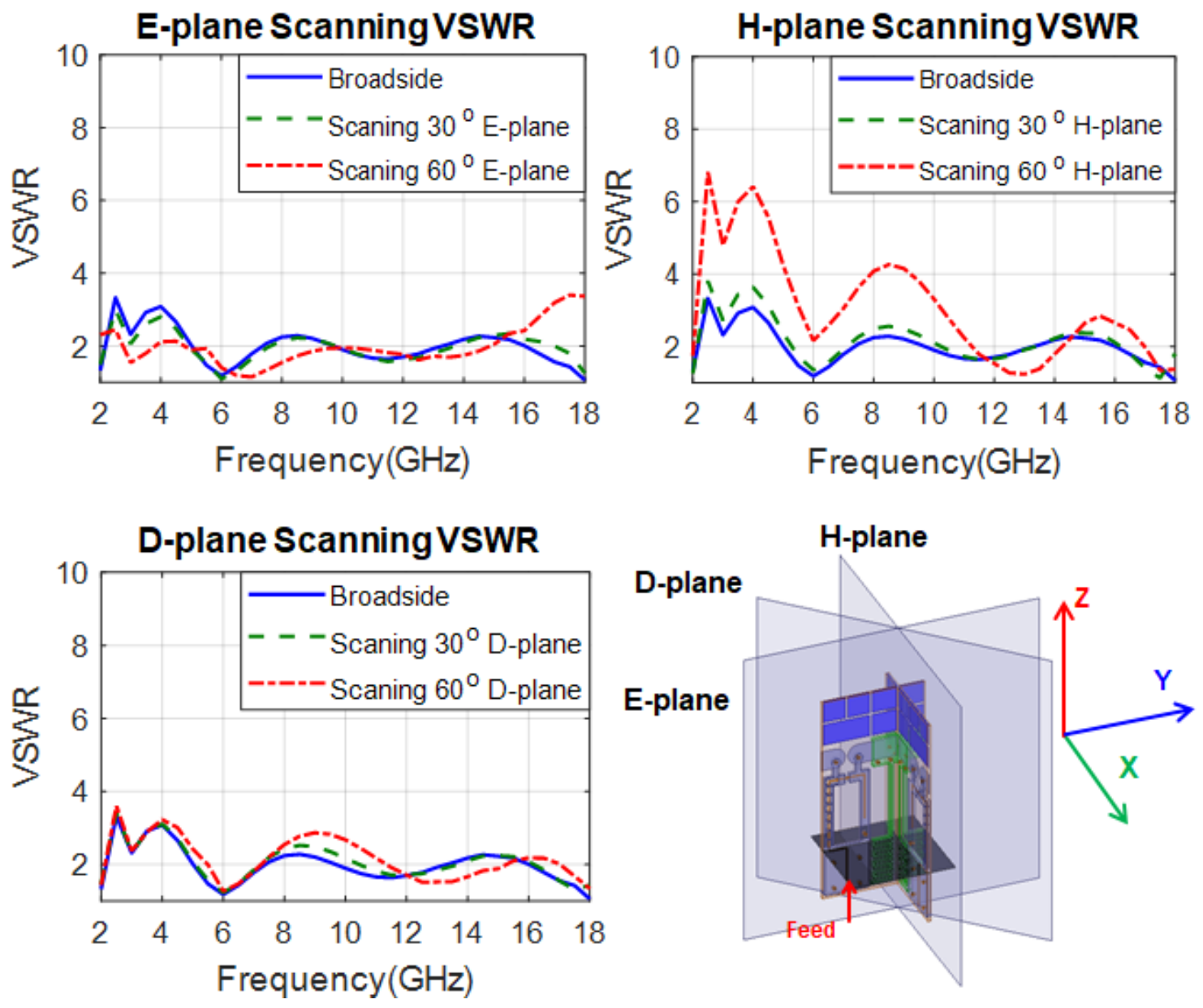

Figure 2.4: Simulated Performance of 9:1 TCDA. 


\subsubsection{Design Construction and Measured Results}

\section{Fabrication of 9:1 TCDA}

The $11 \times 11$ prototype was fabricated with a minimum metal tolerance of 0.15 $\mathrm{mm}$ (6 mil) in accordance with standard low cost PCB processes. The antenna board is constructed from two layers of DiClad 880 with $\varepsilon_{r}=2.2$. The fabricated dual-polarized array in Fig. 2.5 was constructed in an "egg-crate" arrangement with consideration towards structural stability. Notches were cut into the dielectric boards to allow an orthogonal fit between the layers. The fabricated ground board was milled from a metalized 60 mil FR4 board with cutouts for securing the antenna cards.

\section{Measured Results for the 9:1 TCDA}

The fabricated $11 \times 11$ dual-polarized array was measured and characterized at Florida International University. To see the performance of the finite array, multiple antenna elements were measured around the fabricated prototype. The measured active VSWR can be found in Fig. 2.6(left), with comparison to simulations. The measured broadside gain of the array can be found in Fig 2.6(right), with more than $20 \mathrm{~dB}$ of polarization purity. The aperture area is $<\lambda / 2$ at the lower frequencies, causing the reference directivity equation of $D=4 \pi A / \lambda^{2}$ to underestimate the gain (See standard reference directivity in [Dir14]. Hence, the measured gain exceeds the nominal expression for directivity at the lower frequency band. Further, the measured patterns in Figs. 2.7 and 2.8 validate the simulations, showing beam steering down to $60^{\circ}$ from broadside in the principle $\mathrm{E} / \mathrm{H}$ planes using the Active Element Pattern (AEP) method [Poz94]. The patterns are measured at 2.5, 8, 12, and 18 $\mathrm{GHz}$ with close relation to simulated patterns. 


\section{Full Dual Polarized Array Prototype}
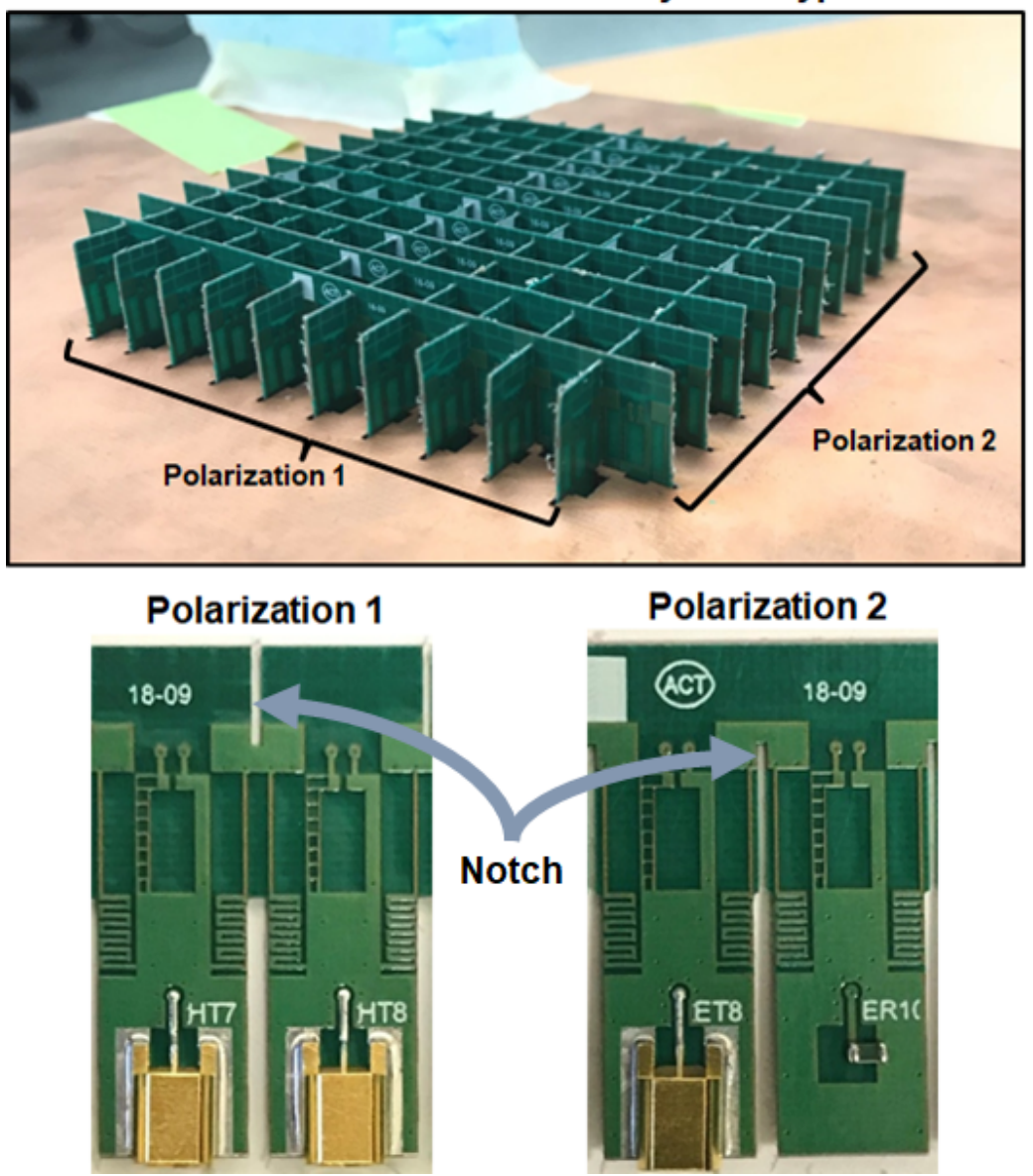

Figure 2.5: Fabricated 9:1 TCDA with notch cut-outs shown for each polarization.
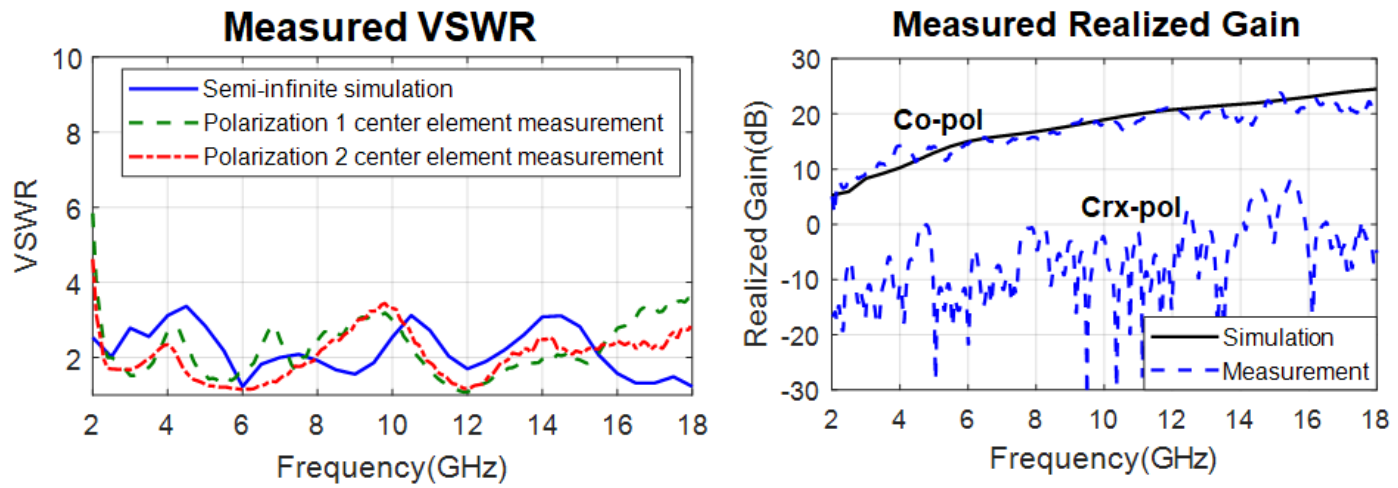

Figure 2.6: Measured VSWR and broadside gain of center element. 

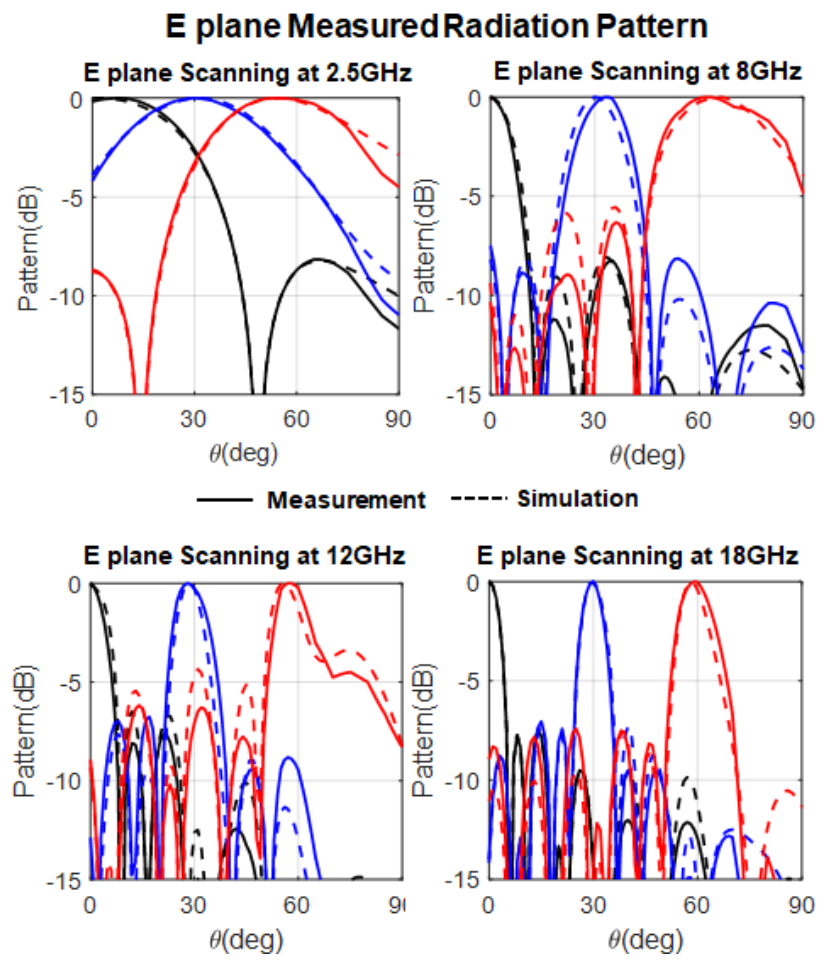

Figure 2.7: Measured E-Plane Pattern of 9:1 TCDA.
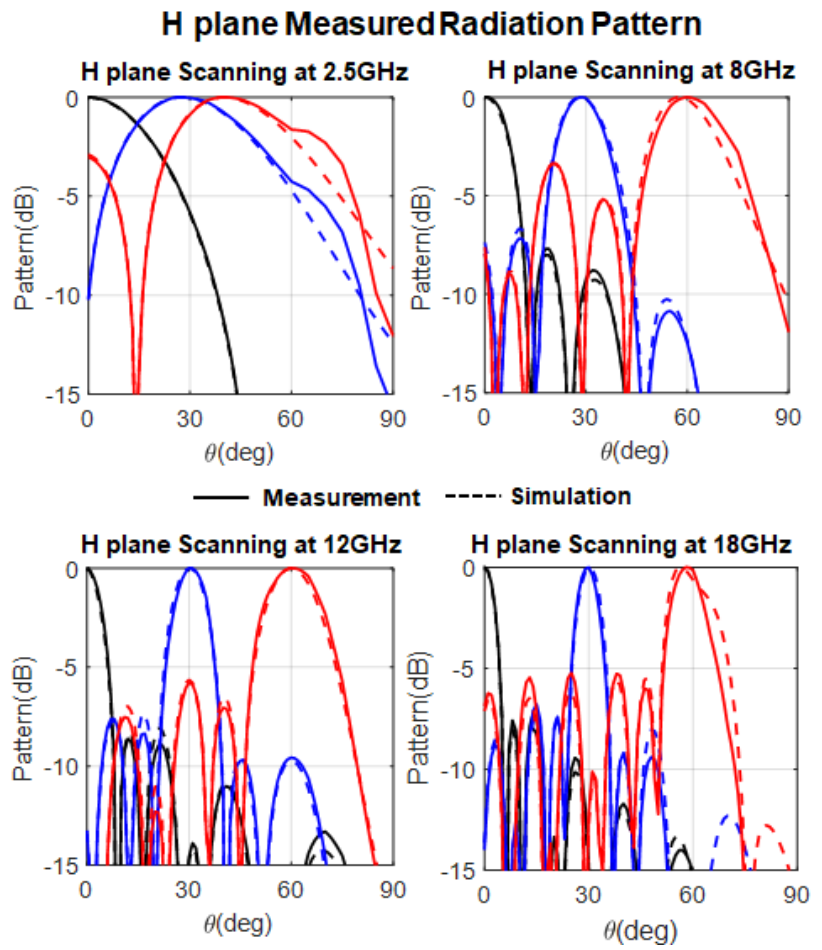

Figure 2.8: Measured H-Plane Pattern of 9:1 TCDA. 


\subsection{An Ultra Wideband Array for Small Platforms}

As discussed in Chapter 1, TCDA have great potential airborne platforms due to their large bandwidths that enable multi-functional missions. The 12:1 presented dual-polarized array presented here improves on the previously mentioned design with 12:1 bandwidth and scanning capability down to $60^{\circ}$, as depicted in Fig. 2.9. The presented low-profile UWB radiating aperture demonstrates bandwidthgain-scanning performance such that it simultaneously supports requirements for an increasing set of required antenna functions in a band of interest to airborne applications. Specifically, its operation from $190 \mathrm{MHz}$ to $2.3 \mathrm{GHz}$ makes it a prime candidate for Foliage Penetrating (FOPEN) radar applications. This application requires a broad range of frequencies to have a tunable range and resolution, with wide spatial scanning to quickly obtain information of a forested landscape. Improving on the performance of past works [MSV12b, PV16a], the array design presented here combines FSS superstrates and resistive loading for an optimum dual polarized prototype. This array introduces 1) improved bandwidth through loading a resistive card (R-Card) in the substrate, 2) an FSS superstate optimized for $60^{\circ}$ scanning and high radiation efficiency, 3) and a streamlined dual-polarized fabrication. Simulations are verified with the measured results of a fabricated $7 \times 11$ prototype.

\subsubsection{Design and Simulation of 12:1 TCDA}

\section{Design of 12:1 TCDA}

The TCDA presented in this section is the first of its kind to scan down to $60^{\circ}$ from broadside across a 12:1 bandwidth. We remark that alternative methods for achieving greater bandwidth with ferrite ground planes have been explored. However, they provide the following disadvantages: 1) high losses on the order of 3 


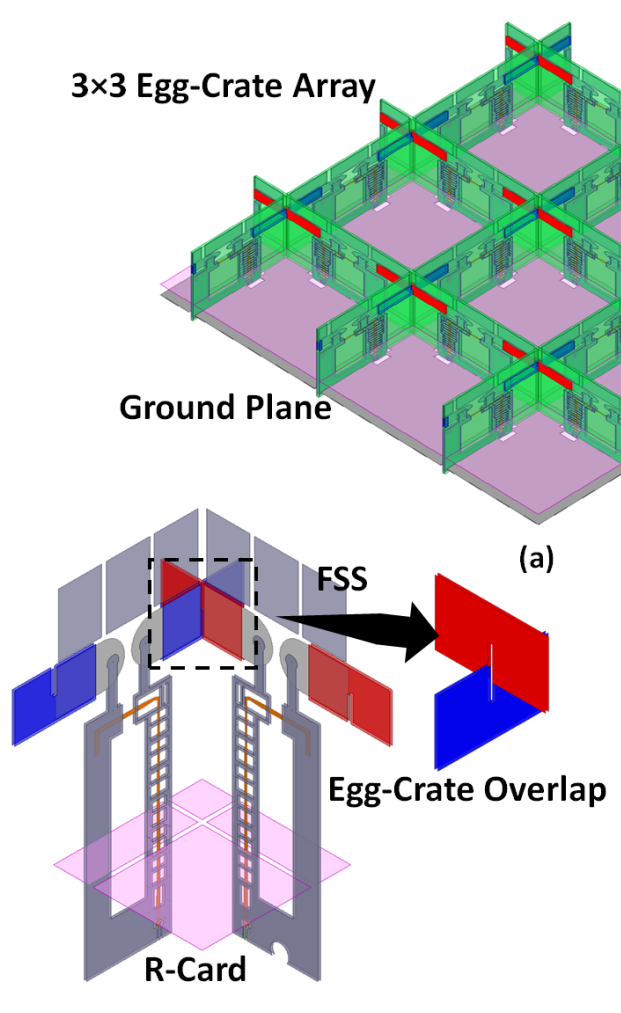

(b)

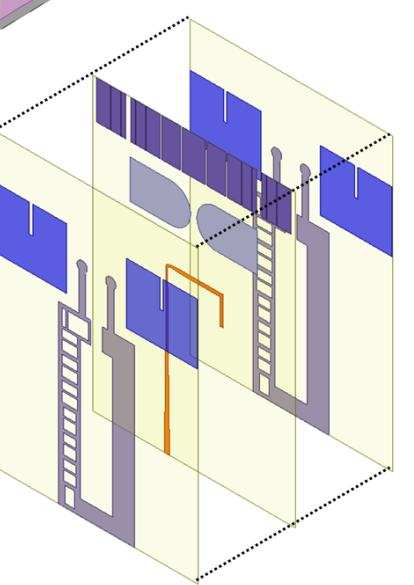

(c)

Figure 2.9: Pictorial representation of the designed TCDA with R-Card (a) $3 \times 3$ array, (b) unit cell, and (c) feed network.

$\mathrm{dB}, 2$ ) anisotropic impedance profiles for dual polarized arrays, and 3) prohibitive weights that preclude them from airborne applications. Alternatively, the array presented here employs resistive substrate loading with an optimized superstrate as in [MSV12a, MSV13, PV16a] to reduce weight and losses. The introduction of a properly designed superstrate above theses arrays reduce these losses significantly [MSV12a]. Notably, the resistive card (R-Card) loading is polarization-insensitive, thus this dual-polarized TCDA retains its symmetry. Further, power handing was considered with the R-Cards. Specifically, the $377 \Omega$ /square Ohmega Technologies material used here was rated for power dissipation capacity of $14 \mathrm{~W} / \mathrm{cm}$. 


\section{Substrate Loading with Resistive Card}

A simple equivalent circuit of dipoles at a distance $h_{1}$ over a ground plane is given in Fig. 2.10(a). The bandwidth of this circuit is limited by the destructive ground plane image at the upper band, corresponding to $f_{\max }=c / 2 h$. This undesirable effect is due to the impedance of the ground plane, that is equivalent to the impedance of a short-circuit transmission line, given by:

$$
Z_{G P}=j \eta \tan (\beta h)
$$

where $\eta$ is the substrate impedance $\left(\right.$ viz. $\left.120 \pi / \sqrt{\epsilon_{r}}\right), \beta$ is the propagation constant in the substrate, and $h$ is the dipole height above the ground plane. It can be seen that when $f_{\max }=c / 2 h$, the impedance of the ground plane goes to zero, and short-circuits the antenna input impedance. As such, the upper frequency cutoff is a function of the height of the array over the ground plane.

To avoid ground plane shorting at $f_{\max }$, an R-Card is placed between the array and the ground plane, as seen in Fig. 2.10(b). To realize efficient R-Card loading, a shunt resistive element is placed at an optimized height $h_{R}$ between the dipoles set at $h_{1}=110 \mathrm{~mm}$ and the ground plane. The relation $h_{1}=h_{R}+h_{2}$ is maintained, with an optimized $h_{R}=1 "$ in this case. It can be seen from Fig. 2.10(c) that the array with no R-card exhibits a resonance that results in a short circuit at about 1.3 $\mathrm{GHz}(f=c / 2 h)$, while the addition of the R-Card nearly doubles the bandwidth. Further, the high impedance surface of the R-Card directs most of the radiated energy upward towards free space and away from the ground plane, which increases

radiation efficiency when used in concert with the optimized FSS superstrate. This substrate loading extends the bandwidth past the limited 9:1 limitation [DSV13b] with an average loss of only $1.37 \mathrm{~dB}$ over the band. 

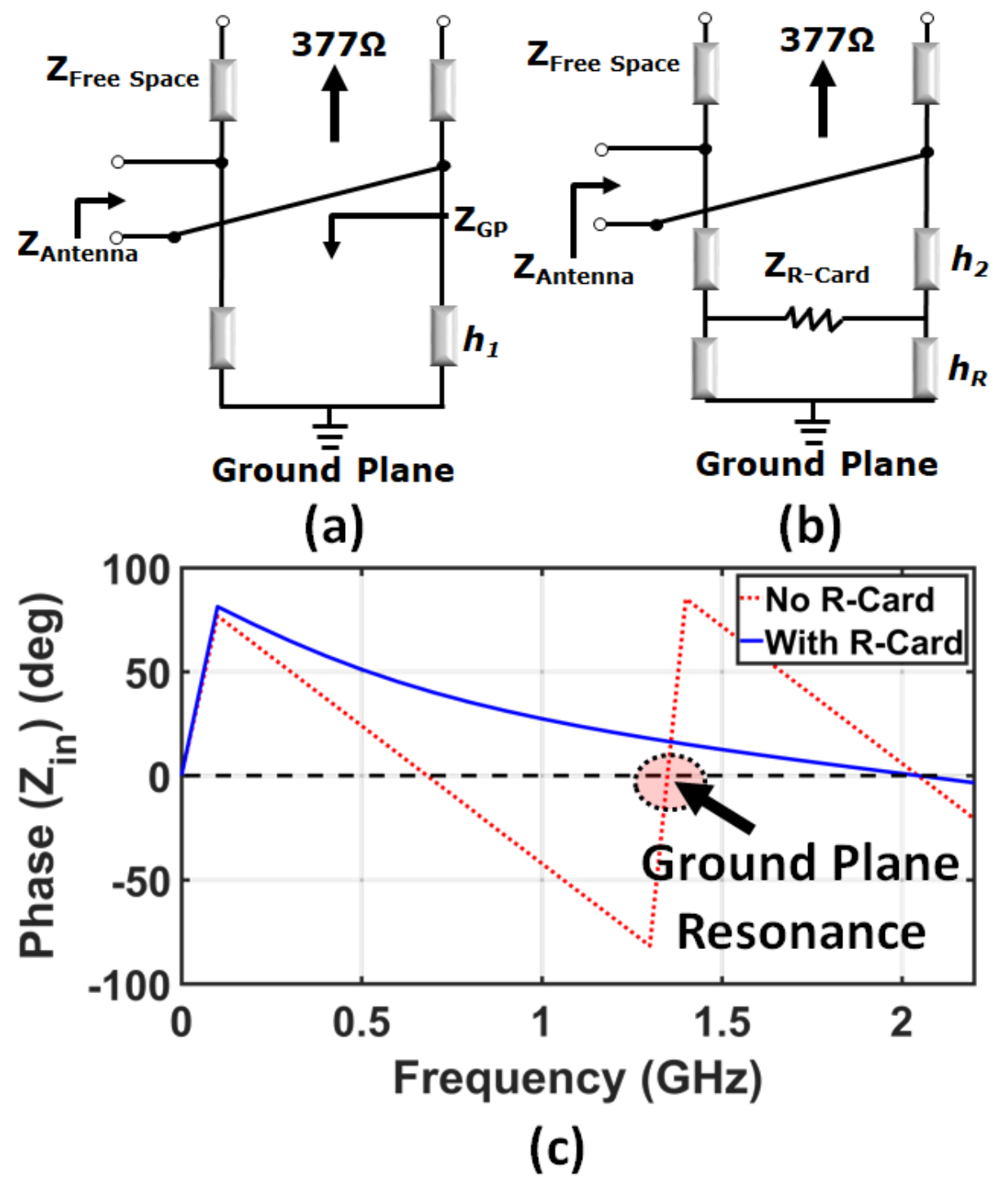

Figure 2.10: (a) Equivalent circuit of ground plane with free space above and (b) the addition of an R-Card. The (c) input impedance of both cases show how the resistive card extends the frequency band where the ground plane resonance is a short circuit. 


\section{2:1 TCDA Design}

The TCDA presented in this section is a dual-polarized array that improves on the bandwidth and designs of [PV16a] and the aforementioned 9:1 array. Notably, the design employs an improved wideband Marchand balun to feed the UWB dipoles without introducing common-mode currents. In addition, an FSS superstrate is designed to enable scanning down to $60^{\circ}$ without exciting surface waves [YGV16]. Further, to reduce fabrication and assembly time, the TCDA and feeds are printed on three layer feed boards. This feeding structure was initially used in the array in the previous section. However, it has only shown over a 9:1 bandwidth as opposed to the 12:1 band demonstrated here. The presented array design was also initially optimized using the equivalent circuits derived in [DSV13c, MSV12a, ASV12]. Again, these equivalent circuits model the tightly coupled dipole as a series L (dipole) and C (coupling), with the superstrate as an equivalent finite length transmission line terminated by the free space impedance of $377 \Omega$. The substrate R-Card was modeled as a shunt resistance in the short circuited transmission line that represents the ground plane, as in Fig. 2.10(b).

\section{Infinite Array Full-Wave Simulation}

The infinite array VSWR for the the E, H, and D planes, with scanning to $60^{\circ}$ is shown versus frequency in Fig. 2.11. The array provides a broadside VSWR $<3$ for $0.19 \mathrm{GHz}$ to $2.3 \mathrm{GHz}$ for an impedance bandwidth of 12:1. As previously discussed, a reduced performance in the $\mathrm{H}$-plane is expected with planar arrays, based on the variation in the Floquet mode impedance in both planes, with a $1 / \cos (\theta)$ term

related to the resistance looking into the H-Plane [Whe64]. However, due to the inherently larger beamwidths at the lower frequencies, it is unnecessary to scan to the low angle of $60^{\circ}$ below $1 \mathrm{GHz}$ with this $7 \times 11$. The realized gain of the infinite 
array unit cell is given in Fig. 2.12 with nearly $30 \mathrm{~dB}$ of cross-polarization isolation across the band in the principle planes, and $>15 \mathrm{~dB}$ when scanning in the diagonal plane.

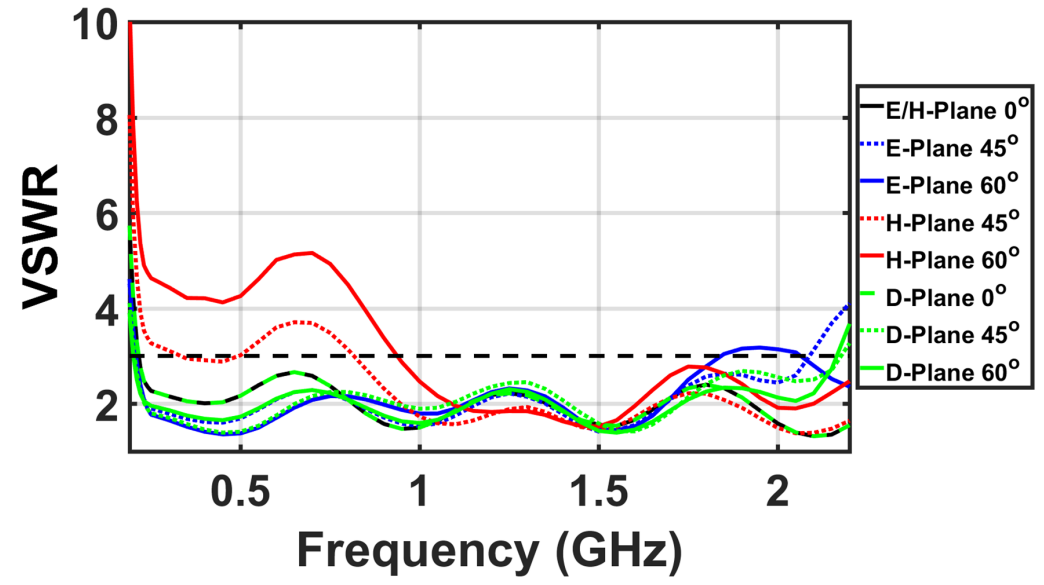

Figure 2.11: Simulated infinite array VSWR in principle planes $(\mathrm{E} / \mathrm{H})$ with scanning down to $60^{\circ}$ from broadside.

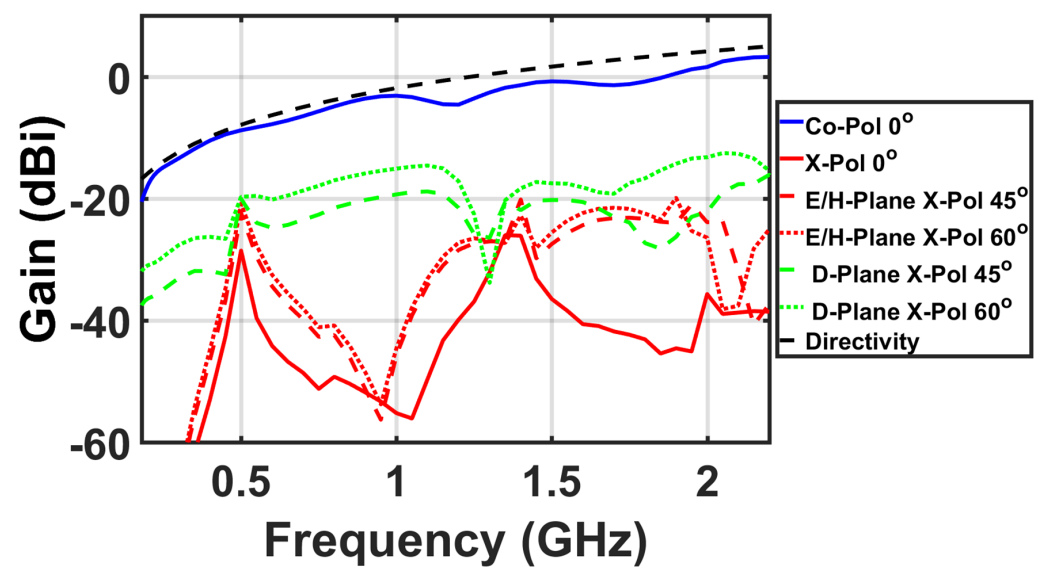

Figure 2.12: Simulated broadside gain of unit cell with more than $30 \mathrm{~dB}$ polarization purity. 


\subsubsection{Finite Array Effects}

The full-wave infinite array simulations discussed above do not account for finite array effects that include edge diffractions and diminished mutual coupling at the array edges. In addition, finite array effects usually result in a narrower operational bandwidth and mismatched peripheral array elements. Therefore, finite array simulations are required to predict more realistic array gain and efficiency. Past works have addressed these finite effects through edge element termination techniques and improved array excitations with finite arrays in a lab setting, as discussed in Section 1.4.1.

These low profile TCDA are of great interest for airborne applications, where space savings are key to their operation. One past TCDA was intended for this application, however it was not designed with its final location in mind, causing extensive losses at lower frequencies when mounted in platform [Tza11]. To maximize the radiation efficiency of the array in its final state, the array presented here was co-designed with typical mounting specifications. Specifically, the airborne platforms' skins can be seen as radomes. These weatherproof enclosures protect the array from the outside environment (e.g., wind, rain, ice, sand, ultraviolet rays), enhance aerodynamics, and provide visual security [Wah09]. An ideal radome is essentially transparent to antenna's operational frequencies, however with UWB arrays and the mechanical needs of an aircraft skin this proves to be difficult to tune over a wide bandwidth. The radome here was modeled with a dielectric constant of $\varepsilon_{r}=6$, an average value of commonly used aircraft skins. Further, to buffer the radiators from peripheral electromagnetic interference (EMI), a variety of shielding techniques were investigated, as depicted by the Walls in Fig 2.13(a). The walls were modeled as either an extension of the radome itself, a perfect electric conductor (PEC) to direct the energy, or a resistive wall to emulate the free space impedance. 
These wall structures on the edges of the array are an integral part of the airborne platform, and must be optimized to increase realized array gain.

To maximize the radiation efficiency of the array in its final state, the array was simulated with the various walls and a 0.23 " thick radome placed 4.2 " from the array face, with an air fill layer, as depicted in Fig 2.13(a). Fig. 2.13(b) shows these results as a normalized gain referenced to the gain of the finite array surrounded by free space. It was concluded that the radome introduces losses $>2 \mathrm{~dB}$ at the upper portion of the band, and the PEC wall introduces the most loss overall. These results inform the cooperative design of the array with the final platform.

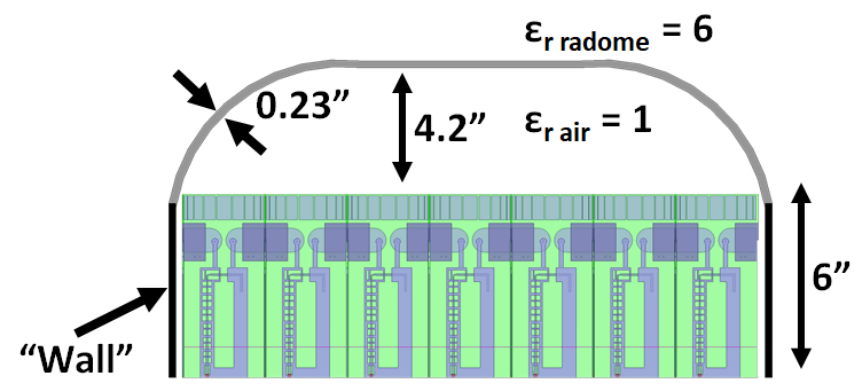

(a)

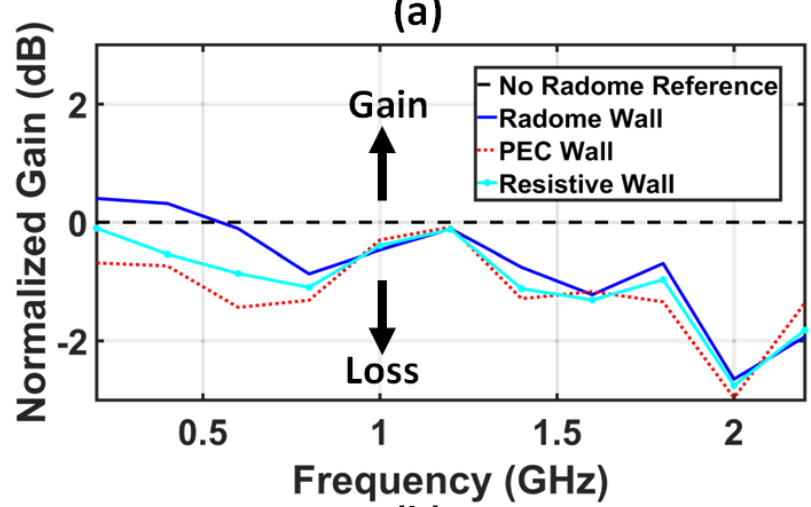

(b)

Figure 2.13: Finite array simulation of 12:1 TCDA showing (a) radome geometry with (b) simulated gain degradation in reference to an array surrounded by free space. 


\subsubsection{Design Construction and Measured Results}

\section{Fabrication of 12:1 TCDA}

The $7 \times 11$ prototype in in Fig. 2.14 was fabricated with a minimum metal tolerance of $0.25 \mathrm{~mm}(10 \mathrm{mil})$ in accordance with standard low cost PCB processes. The exact dimensions of are found in in Fig. 2.15 and Table 2.1. The antenna board is constructed from two layers of Rogers 3003 with $\varepsilon_{r}=3.0$. The fabricated dual-polarized array in Fig. 2.14 was constructed in an "egg-crate" arrangement with consideration towards structural stability. Notches were cut into the dielectric boards to allow an orthogonal fit between the layers as described above. Notably, the fabricated ground board was milled from a metalized 60 mil FR4 board with cutouts for securing the antenna cards. A total of four ground plane sections were joined together with copper tape to form a lightweight, structurally stable ground plane for testing the array.

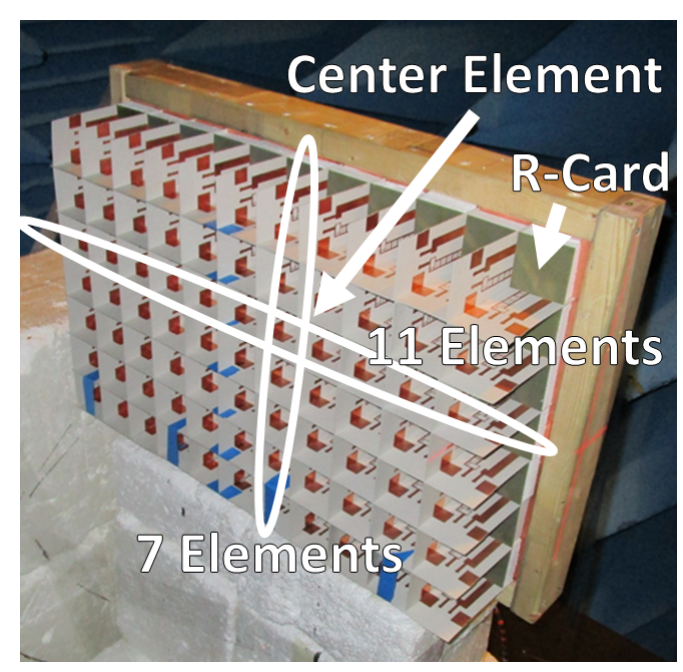

Figure 2.14: Fabricated $7 \times 11$ array secured in anechoic chamber. 


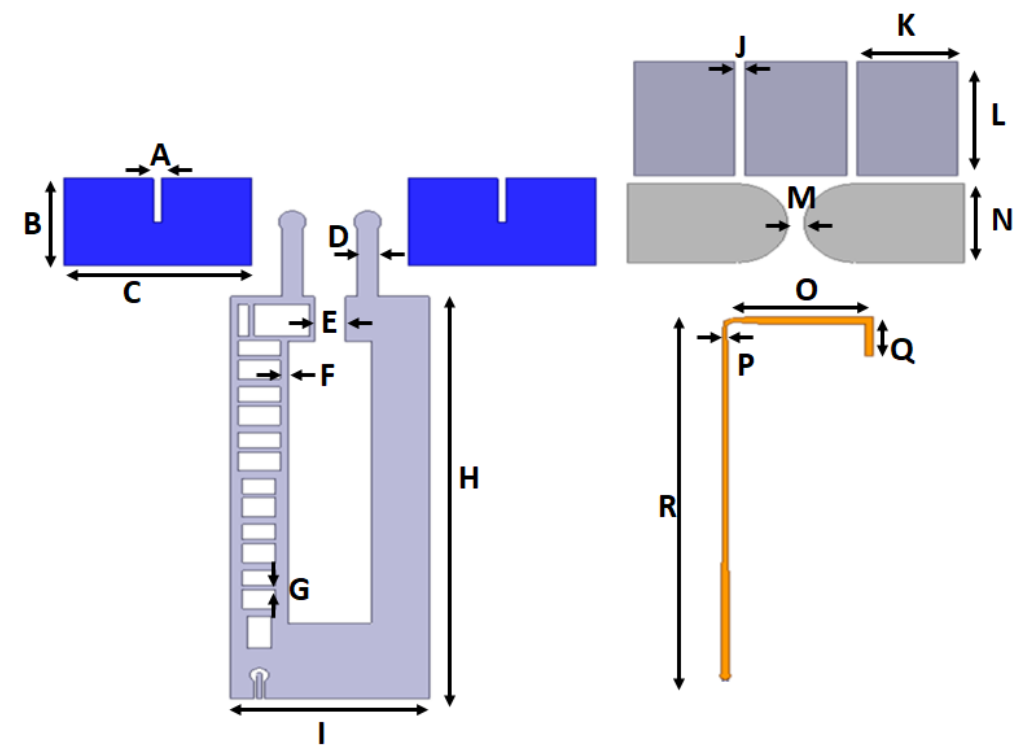

Figure 2.15: Unit cell details of dual polarized D-TCDA used for infinite array simulation. Views of the identical printed metal layers are given. The metal layers are spaced equidistant by substrate layers with $\varepsilon_{r}=3.0$.

Table 2.1: Unit cell details of dual polarized D-TCDA in Fig. 2.15

\begin{tabular}{|c|c|c|c|}
\hline Variable & Dimension $(\mathrm{mm})$ & Variable & Dimension $(\mathrm{mm})$ \\
\hline$A$ & 1.56 & $\mathrm{~J}$ & 2 \\
\hline$B$ & 22 & $\mathrm{~K}$ & 20 \\
\hline$C$ & 37.2 & $\mathrm{~L}$ & 29 \\
\hline$D$ & 4 & $\mathrm{M}$ & 3.25 \\
\hline$E$ & 6 & $\mathrm{~N}$ & 20 \\
\hline$F$ & 1.62 & $\mathrm{O}$ & 22.65 \\
\hline$G$ & 1 & $\mathrm{P}$ & 0.17 \\
\hline$H$ & 102 & $\mathrm{Q}$ & 10 \\
\hline$I$ & 39.25 & $\mathrm{R}$ & 92.26 \\
\hline
\end{tabular}

\section{Measured Results for the 12:1 TCDA}

The fabricated $7 \times 11$ dual-polarized array in Fig. 2.14 was measured and characterized. To see the performance of the finite array, multiple antenna elements were measured around the fabricated prototype. As documented [Tza11], edge effects on peripheral array elements degrade low frequency performance due to a lack of mu- 
tual coupling. However, due to the nature of the resistive loading in the substrate these finite array effects are greatly reduced, with edge elements showing analogous active VSWR between the center and inner elements as depicted in Fig. 2.16(left). To measure the gain of the array, the array was placed in a Tapered Chamber with low frequency cutoff of $180 \mathrm{MHz}$. A power combiner was used to measure individual linear arrays in both polarizations, which were then added together to represent the gain of the full finite array. Following the de-embedding of these combiner losses, a close comparison of the measured gain to finite simulations was demonstrated. The measured broadside gain of the array can be found in Fig 2.16(right), with more than $20 \mathrm{~dB}$ of polarization purity. As typical with UWB TCDA, the aperture area is $<\lambda / 2$ at the lower frequencies, causing the reference directivity equation of $D=4 \pi A / \lambda^{2}$ to underestimate the gain (See standard reference directivity in [Dir14]. Hence, the measured gain exceeds the nominal expression for directivity at the lower frequency band. Further, the measured patterns in Fig. 2.17 validate the simulations, showing beam steering down to $60^{\circ}$ from broadside in the principle $\mathrm{E} / \mathrm{H}$ planes with close relation to simulated patterns using the Active Element Pattern (AEP) method [Poz94].
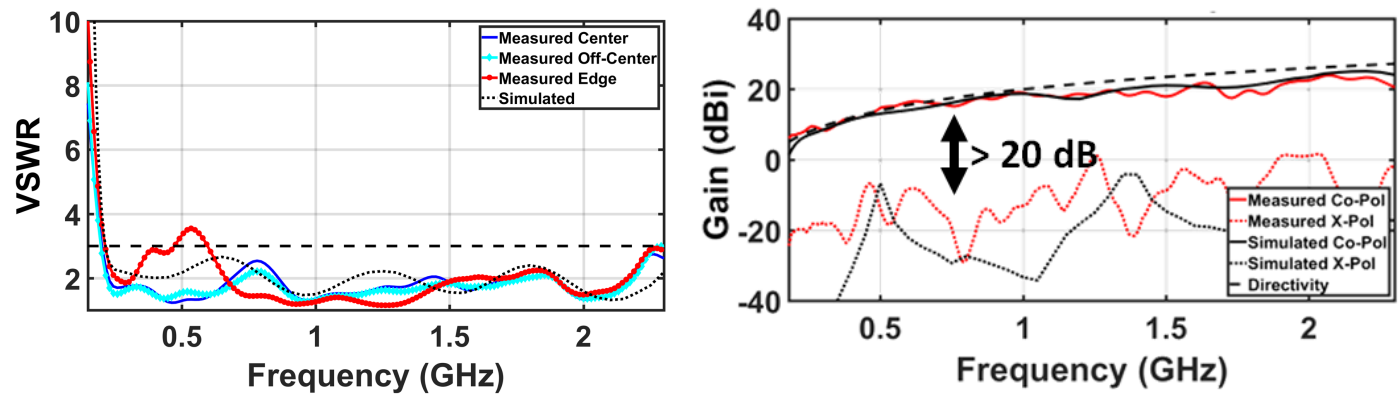

Figure 2.16: (Left) Measured active VSWR of array elements. (Right) Measured broadside gain of finite array with comparison to simulation 


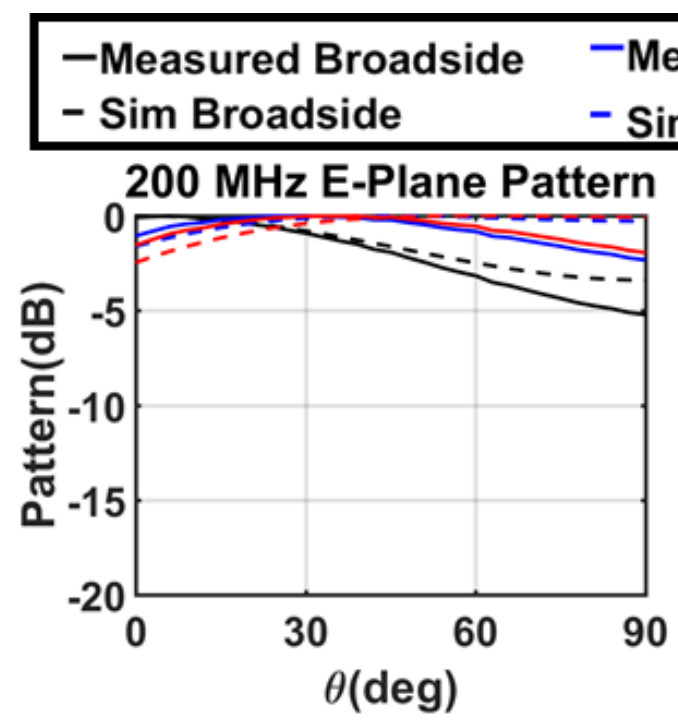

- Measured $45^{\circ}-$ Measured $60^{\circ}$

$\operatorname{Sim} 45^{\circ}-\operatorname{Sim} 60^{\circ}$
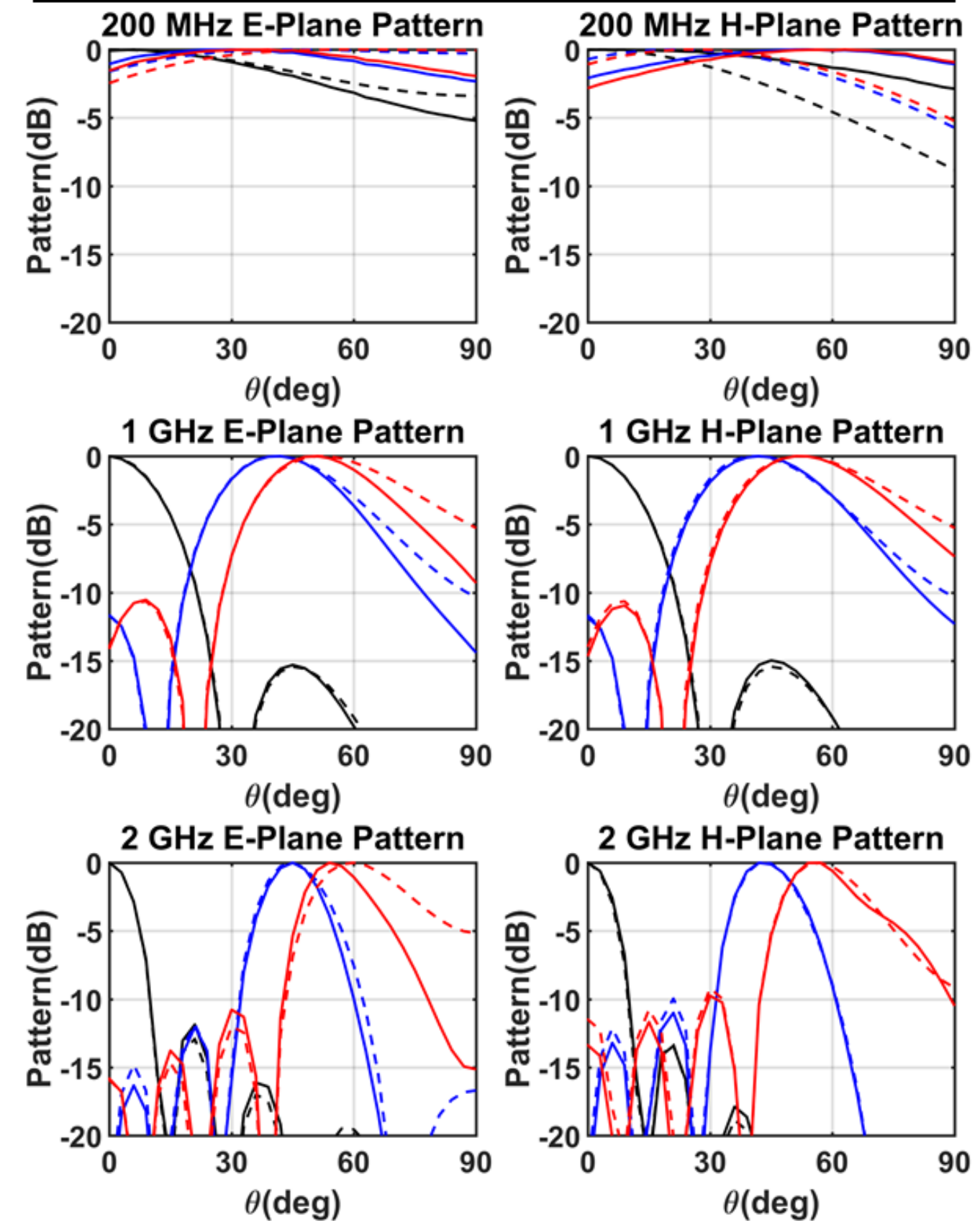

Figure 2.17: Measured E-Plane (left) and H-Plane (right) patterns of a center element showing scan capability down to 60 degrees using the Active Element Pattern Method. 


\subsection{An Extremely Wideband Array for Small Platforms}

In this section, we further expand on the TCDA concept to implement a very thin $(\lambda / 13.5$ thick at the lowest frequency of operation) extremely wideband (EWB) array. For the first time, the TCDA achieves a contiguous 58:1 broadside impedance bandwidth (VSWR $<3$ ) with an average radiation efficiency of $72 \%$ across the band. The array is depicted in Fig. 2.18, and includes an FSS superstrate to achieve scanning down to $60^{\circ}$ from broadside across the entire band.

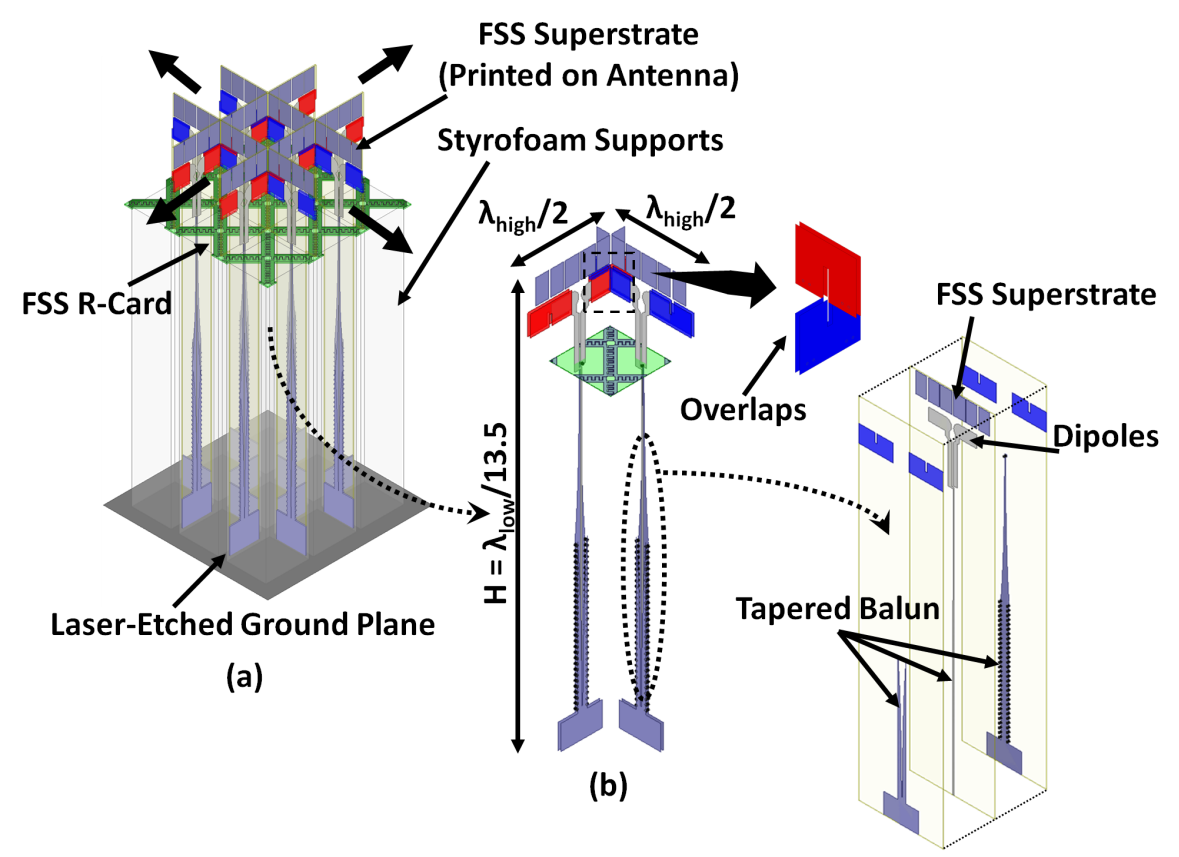

(c)

Figure 2.18: Pictorial representation of the EWB TCDA with FSS R-Card (a) showing the a $4 \times 4$ section of the finite array operating from 0.13-7.63 GHz. The (b) $24.5 \mathrm{~mm} \times 24.5 \mathrm{~mm}$ unit cell is detailed with capacitive overlaps and (c) the tapered balun stripline feed layers.

Notably, the element-to-element separation of the adjacent array elements is only $\lambda / 92$ at the low frequency of operation (viz. $130 \mathrm{MHz})$. The array elements are periodically spaced at $\lambda_{H i g h} / 2$ apart, where $\lambda_{H i g h}$ corresponds to the highest operational 
scan frequency of $6 \mathrm{GHz}$. As such, a 58:1 broadside bandwidth is achieved without grating lobes. Two other novelties are introduced in this array to achieve such a large bandwidth: 1) Introduction of a novel frequency selective surface resistive card (FSS R-Card) within the substrate. The FSS R-Card plays the crucial role of cancelling the ground plane reflection when the distance between the array and ground plane are multiples of $\lambda_{H i g h} / 2$ across the band; 2) A new wideband balun comprised of an exponentially tapered stripline feed. Notably, bandwidth and efficiency are verified with measured data.

\subsubsection{Design and Simulation of EWB TCDA}

The presented dual-polarized TCDA employs a metal FSS superstrate for low-angle scanning [YGV16]. The array profile is only $\lambda_{\text {Low }} / 13.5$ thick, more than $3 \times$ shorter than a 10:1 Vivaldi array with $\lambda_{H i g h} / 2$ spacing [LKV18]. As in past TCDA, the cross-polarized dipole elements are not fed concentrically, but instead the feed cards intersect at the ends of the dipoles. Again, this design is printed coherently on the three-layer feed boards to save fabrication and assembly time. The dipole arms reside in the center layer and the capacitive sheets lie on the outer layers to create the coupling needed to achieve the current sheet effect. The implementation of the FSS resistive card builds on the concept presented in [MSV12a] and serves to cancel the negative effects of periodic ground plane reflections. In doing so, the bandwidth is extended to $58: 1$ with an average loss of only $-1.42 \mathrm{~dB}$ across the band.

\section{Frequency Selective Resistive Card}

The new resistive FSS was placed within the substrate to extend the capabilities of the TCDA to an impressive 58:1 achievable impedance bandwidth. Fig. 2.19 
shows that inside the band of interest there are six ground plane resonances to be addressed. Drawing on past implementations of the frequency selective restive card [MSV12a], this novel R-Card is frequency dependent and tuned for maximum radiation efficiency across the entire band by only attenuating the ground-reflected waves at the frequencies where the resonances occur. In order to expand the bandwidth of the R-Card, a stacked card implementation of 2 and 4 cards was investigated, as shown in Fig. 2.20. Others have utilized this multi-layered resistive loading technique to achieve up to $33: 1$ bandwidths with efficiencies greater than $50 \%$ with fragmented arrays $\left[\mathrm{MBL}^{+} 11\right]$.

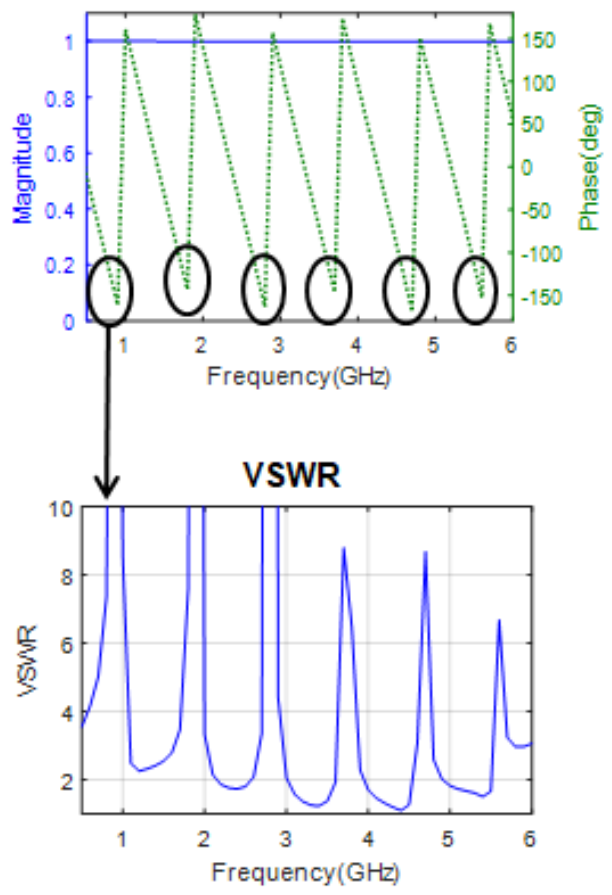

Figure 2.19: Floquet port reflection response with plane-wave excitation of TCDA unit cell without R-Card.

The resistive cards were optimized using a unit cell of periodic boundaries with an ideally fed dual polarized TCDA without the balun feed. The differential lumped port between the dipole terminals allowed for fast computational optimization of the 
designs. First, the $\lambda_{6 \mathrm{GH} z} / 2$ dipoles were placed at a set height above the ground plane, to see the magnitude and phase of reflection coefficients of the dipoles in presence of a ground plane. As expected, the ground plane reflections appeared as $\mathrm{a}+180^{\circ}$ phase shift that destructively canceled the upward radiating wave for an efficiency of $0 \%$. To counter this reflection, the number, resistance value, and height of each R-card implementation was tuned to optimally reduce the magnitude of the ground reflections and steer the energy to a lower resistance in the superstrate, for $>50 \%$ efficiency at these narrow bands. The 4-layer arrangement produced the desired bandwidth for the intended TCDA radiator, but with wideband losses over the whole of the band. Therefore, the FSS R-Card in Fig. 2.20(d) was investigated to mimic the higher order effect of four stacked R-Cards with minimal losses.

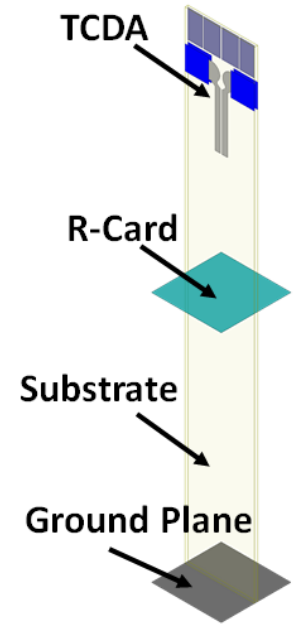

(a)

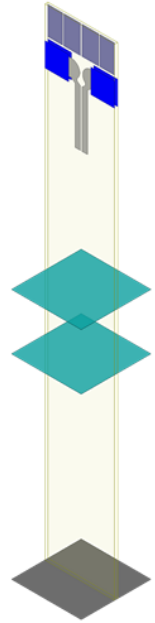

(b)

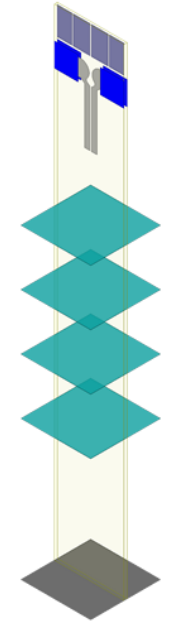

(c)

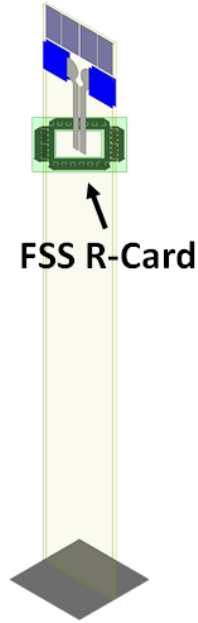

(d)

Figure 2.20: Progression of the 58:1 enabling R-card with the (a) 1-Layer [MSV13, PV16a] (b) 2-Layer (c) 4-Layer $\left[\mathrm{MBL}^{+} 11\right]$, and (d) Optimized FSS Designs (this work).

Typical of FSS with ring-type geometry, this resistive FSS has a band-stop response [Mun05], with additional optimized teeth for a multi-notch filter behavior. Further, the resonant frequencies of the FSS are designed to cancel the negative 


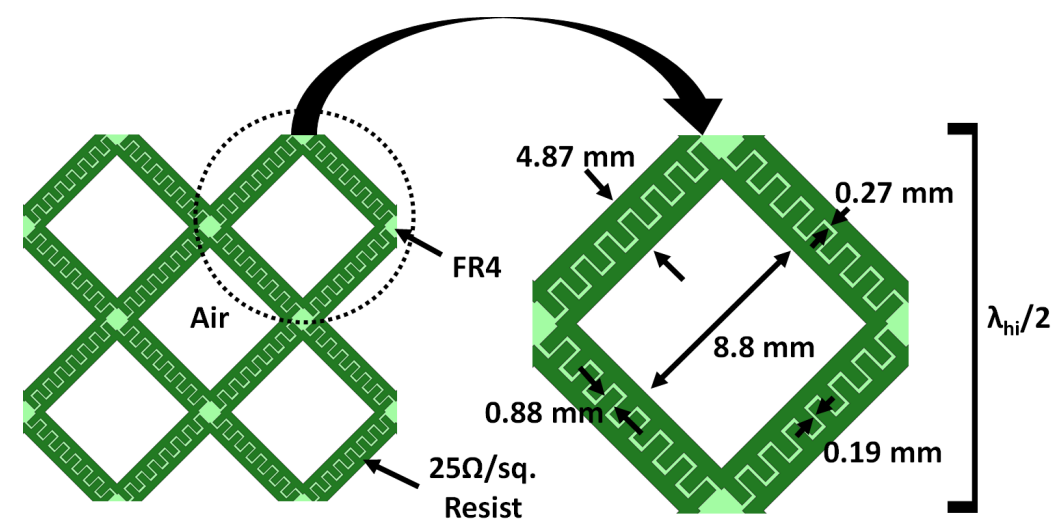

(a)

(b)

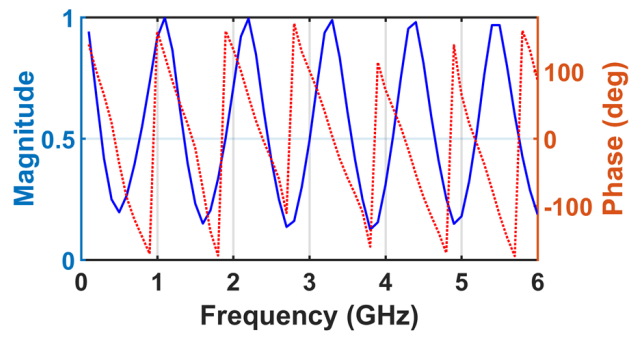

(c)

Figure 2.21: Pictorial representation of (a) the novel 58:1 enabling FSS R-Card showing square loop design with (b) unit cell dimensions of the notched teeth. (c) Simulated $S_{11}$ magnitude and phase response of a plane wave reflected from an infinite ground plane in presence of the FSS R-Card

effects of the ground reflections at periodic heights of $N \lambda / 2$ (where $\mathrm{N}$ is the number of corresponding periodic high frequency ground plane reflections). Doing so, the bandwidth is extended to $58: 1$ with an average loss of $1.42 \mathrm{~dB}$ over the band at broadside. The square loop's radius, line widths, and height above the ground plane were tuned as design variables to achieve the intended filtering response. The frequency response of the final design is depicted in Fig. 2.21(c), and shows attenuated response only at the periodic frequencies that correspond to the cyclical ground plane short circuits, viz. $N \lambda / 2$. The final FSS R-Card design is depicted in Fig. 2.21. As compared to past resistive FSS [FAZ $\left.{ }^{+} 13, \mathrm{MSV} 12 \mathrm{~b}\right]$, this FSS R-Card operates over a wider bandwidth due to its higher order response from the optimized gaps and teeth. Theoretically, the bandwidth a resistive loaded TCDA can be even 
further improved by increasing the order of the FSS R-Card with smaller loops to resolve higher frequency ground reflections. However, the height of the dipole above the ground plane is a function of the lowest frequency of operation, while the highest is determined grating lobes and array spacing. Therefore, as the lateral dimensions shrink and vertical dimensions stay constant the bottleneck of the design becomes mechanical considerations and fabrication tolerances.

\section{Extremely Wideband Balun Feed}

To excite this 58:1 aperture, two new wideband feeds were designed and evaluated against the Marchand balun, as illustrated in Fig. 2.22. As discussed, previous TCDA employed a Marchand Balun to feed the dipole arms. Indeed, the UWB performance of these TCDA owe to the integration of a Marchand Balun [DSV13c] that has proven to be a higher order matching network across 14:1 bandwidths [MSV12a].

However, as depicted in Fig. 2.22(d), the Marchand Balun cannot perform over a 58:1 bandwidth. As an alternative, the co-planar waveguide (CPW) to co-planar stripline (CPS) balun in Fig. 2.22(b) was investigated. This feed acted as an effective balun with cancellation of the common-mode currents across the entire bandwidth, but lacked the impedance transformation and transmission efficiency of the tapered balun. The chosen balun in Fig. 2.22(c) draws from [BQG16] with a stripline configuration of exponentially tapered feed and ground traces. This balun cancels common-mode currents while transferring the input impedance from 188 Ohms [ASV12] at the dipole to 50 Ohms at the connector in a total aperture profile of only $\lambda_{\text {Low }} / 13.5$. 


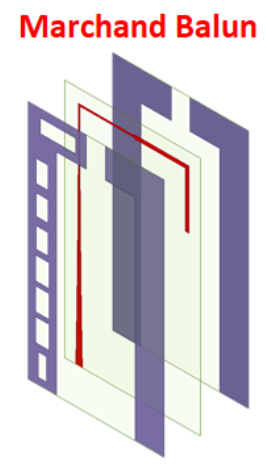

(a)

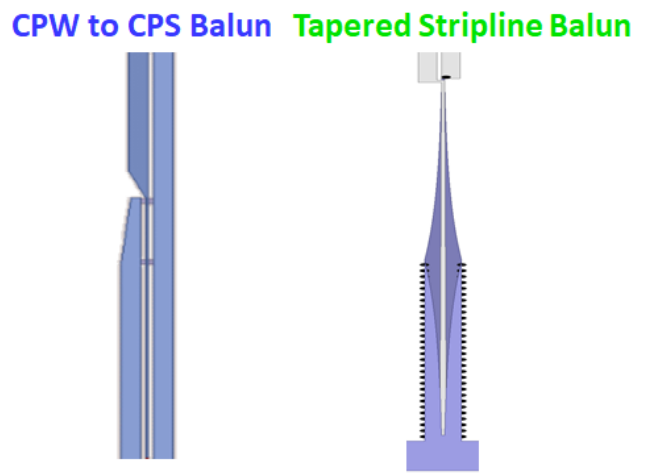

(b)

(c)

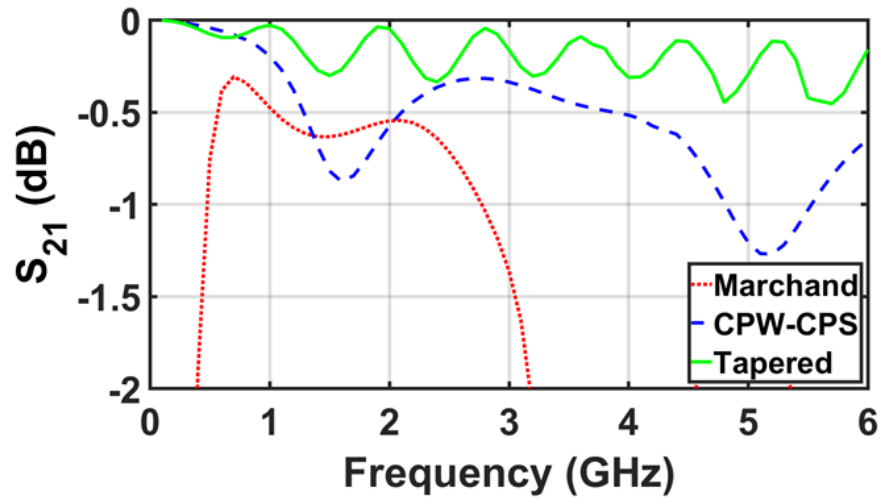

(d)

Figure 2.22: Simulated (a) Marchand balun, (b) co-planar waveguide (CPW) to co-planar stripline (CPS) balun, and (c) tapered balun feeds investigated to excite the EWB dipoles, with (d) a comparison of insertion loss.

\section{TCDA with FSS R-Card Equivalent Circuit}

The EWB array design was initially optimized using the aforementioned equivalent circuits derived in [DSV13c, MSV12b, ASV12]. Further, as shown in Fig. 2.23(a) a tapered feed line and series RLC substrate were included to represent the feed and FSS R-Card. By optimizing the circuit values, a close comparison to the full-wave model was achieved. It should be noted that these ideal transmission line equivalents are not 1:1 design guides, but rather tools used characterize the input impedance response by tuning characteristic impedances, lengths and coupling terms. 


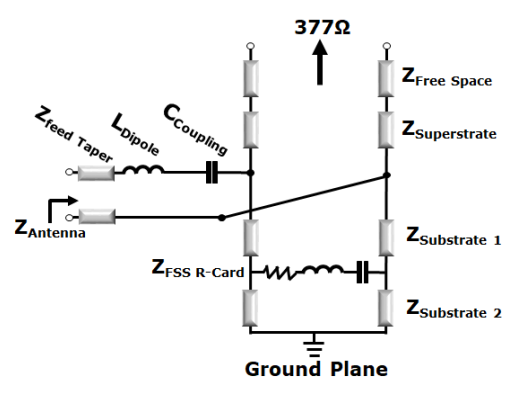

(a)

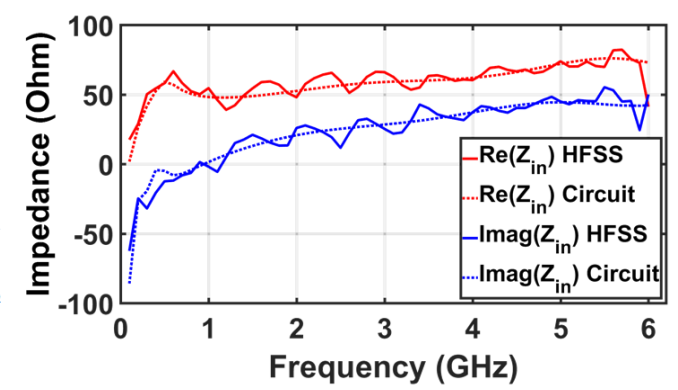

(b)

Figure 2.23: (a) Equivalent TCDA circuit with FSS R-Card loading and (b) input impedance verification of the circuit model versus HFSS.

\section{Verifying the 58:1 TCDA Infinite Array Performance}

An infinite array simulation was used to represent the $12 \times 12$ finite element array shown in Fig. 2.18. The infinite array VSWR for the principle planes, with scanning to $60^{\circ}$ is shown versus frequency in Fig. 2.24(a). The array was designed for a VSWR $<3$ for $0.13 \mathrm{GHz}$ to $6 \mathrm{GHz}$ for an impedance bandwidth of 46:1 with scanning without grating lobes. Again, the reduced performance in the low frequency Hplane VSWR is expected when scanning to low angles due to the $1 / \cos (\theta)$ free space impedance, hence the mismatch around $2 \mathrm{GHz}$ at $60^{\circ}$. The array is characterized by its near theoretical gain and average principle plane polarization purity of $40 \mathrm{~dB}$ as Fig. 2.24(b) depicts. As expected, the D-plane cross-polarized gain level rises when scanning, but the EWB TCDA still exhibits greater than $20 \mathrm{~dB}$ cross-polarization suppression across the band. The simulated radiation efficiency of the infinite array unit cell is $72 \%$ on average, with the lowest efficiency of $50 \%$ in the narrowband of the first ground plane resonance around $1 \mathrm{GHz}$. This narrowband efficiency drop is due to the high magnitude of the reflection coefficient present at the fist ground reflection (phase change in red), as depicted in Fig. 2.21. In theory, this efficiency can be improved by further tuning the first resonance of the FSS R-Card to have a lower magnitude response at the first ground plane resonance. 


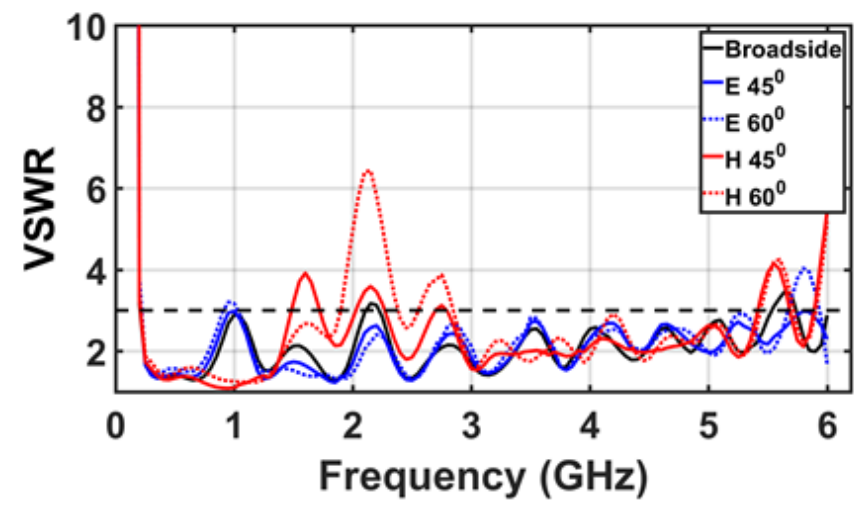

(a)

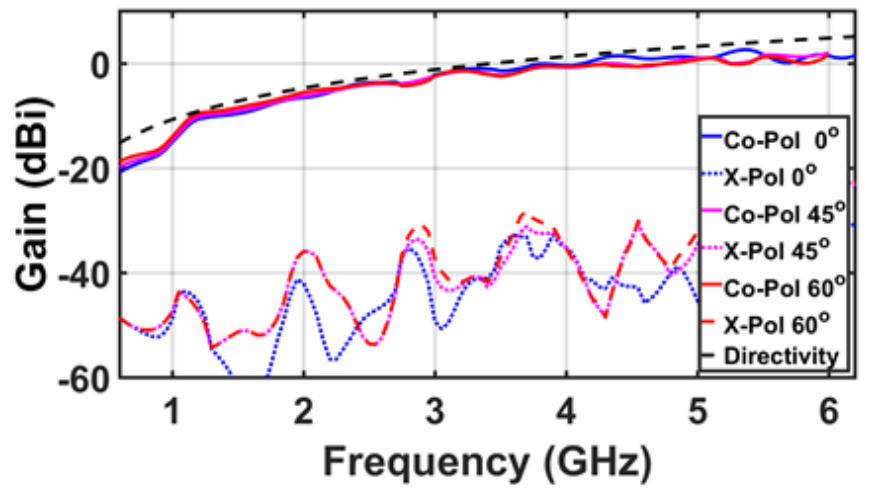

(b)

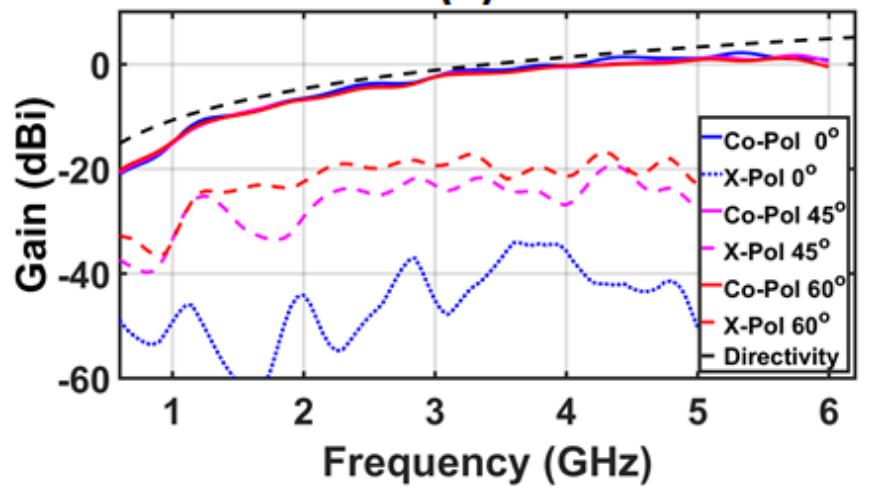

(c)

Figure 2.24: (a) Simulated infinite array active VSWR in principle planes $(\mathrm{E} / \mathrm{H})$ with scanning down to $60^{\circ}$ from broadside. (b) Realized gain in principle planes $(\mathrm{E} / \mathrm{H})$ for simulated unit cell with scanning down to $60^{\circ}$ from broadside. (c) Realized gain in D-plane for simulated unit cell with scanning down to $60^{\circ}$ from broadside. 

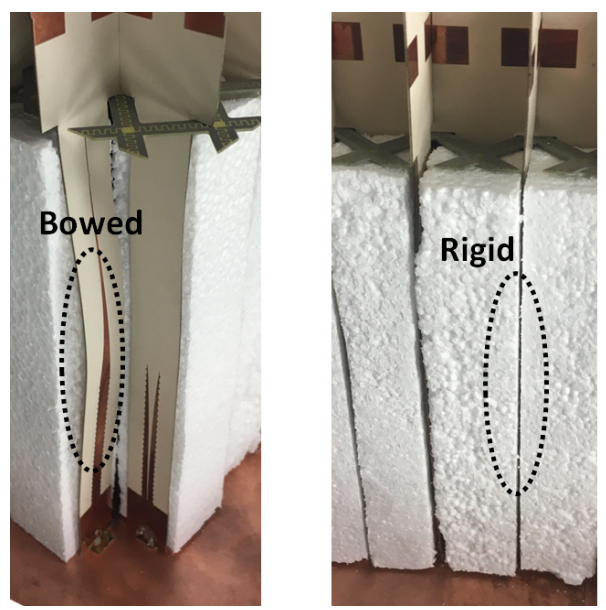

Figure 2.25: Fabricated antenna cards with bowed feeds (left) and with Styrofoam supports (right).

\section{Material Considerations}

The array was designed and fabricated using two layers of 10 mil thin Rogers 3003 substrate for low loss and low cost PCB fabrication. The electrical properties of Rogers $3003(\tan \delta=0.001)$ help in reaching the arrays impressive efficiency over such a wide bandwidth. However, in practice the mechanical properties of this Polytetrafluoroethylene (PTFE) material caused bending and bowing in some of the antenna cards, as seen in Fig. 2.25. These fabrication imperfections can result in inconsistent elements that reduce element-level coupling and impedance matching. Further, bending in the antenna cards can increase cross-polarized gain levels up to $30 \mathrm{~dB}$ for even $1^{\circ}$ of misalignment [Lud73]. To reduce this effect, the $23 \mathrm{~mm} \times 23 \mathrm{~mm} \times 134.5 \mathrm{~mm}$ Styrofoam blocks placed in between the cards to set the FSS R-card height doubled as mechanical supports for the array. The array in Fig. 2.26 is shown without these Styrofoam blocks for clarity of the design. For future consideration, a prototype implemented on a mechanically rigid substrate, such as the woven glass ceramic Rogers 4003, would be a better candidate for fabrication. 


\subsubsection{Fabrication and Measured Results}

The $12 \times 12$ dual-polarized array prototype in Fig. 2.26 was fabricated and measured. The FSS metal superstrate was printed on the vertical antenna cards for optimized scanning performance. The antenna board was constructed from two layers of Rogers 3003 with $\varepsilon_{r}=3.0$. As before, the fabricated ground board was milled from a metalized 60 mil FR4 board with cutouts for securing the antenna cards. A total of four ground plane sections were joined together with copper tape to form a lightweight, structurally stable and resonance free ground plane for testing the array.

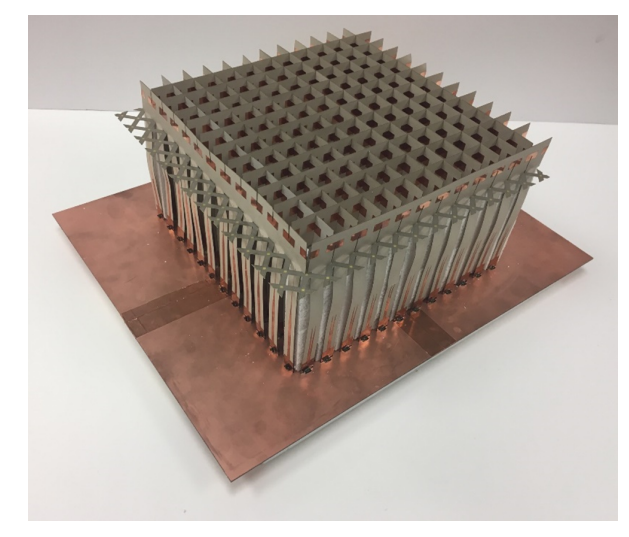

Figure 2.26: Fabricated $12 \times 12$ array.

To accurately implement the prototype, the tolerances of commercial PCB manufacturing were constantly considered in the design process, where metal thickness and via misalignment result in a significant change from the ideal design. This design used 10 mil diameter vias and a minimum metal tolerance of $0.1524 \mathrm{~mm}(6 \mathrm{mil})$ in accordance with standard low-cost commercial printed circuit board (PCB) processes. To ensure structural stability, the fabricated dual-polarized array was constructed in an "egg-crate" arrangement with notches cut into the dielectric boards for an orthogonal fit between the layers. For ease of fabrication, the array is designed with no direct electrical connection or soldering required at the joints. 


\section{Fabrication of FSS Resistive Card}

The fabricated FSS R-Card consisted of $25 \Omega /$ square Omega-Ply material printed on a 20 mil thin FR4 substrate, as depicted in Fig. 2.27. An air gap was intentionally routed from the inner radius of the square loop to allow placement of the antenna cards through the FSS. Styrofoam supports were used to place the FSS R-Card at the desired height of $134.5 \mathrm{~mm}$ above the ground plane. Note the FSS R-Card was fabricated and placed in a symmetric orientation to the dipoles, to allow equal filtering response for both polarizations.

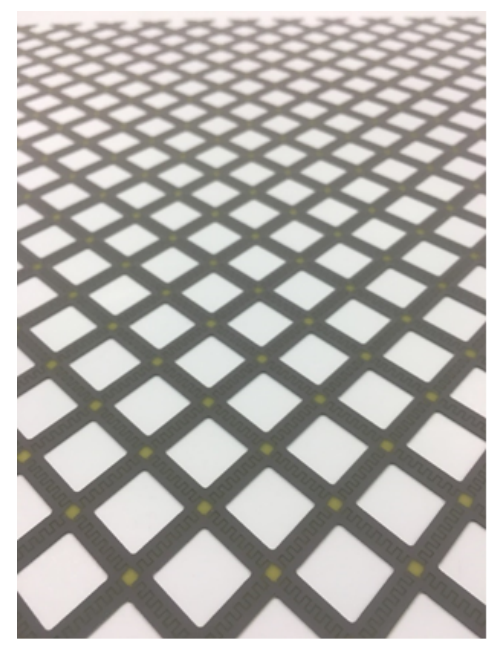

(a)

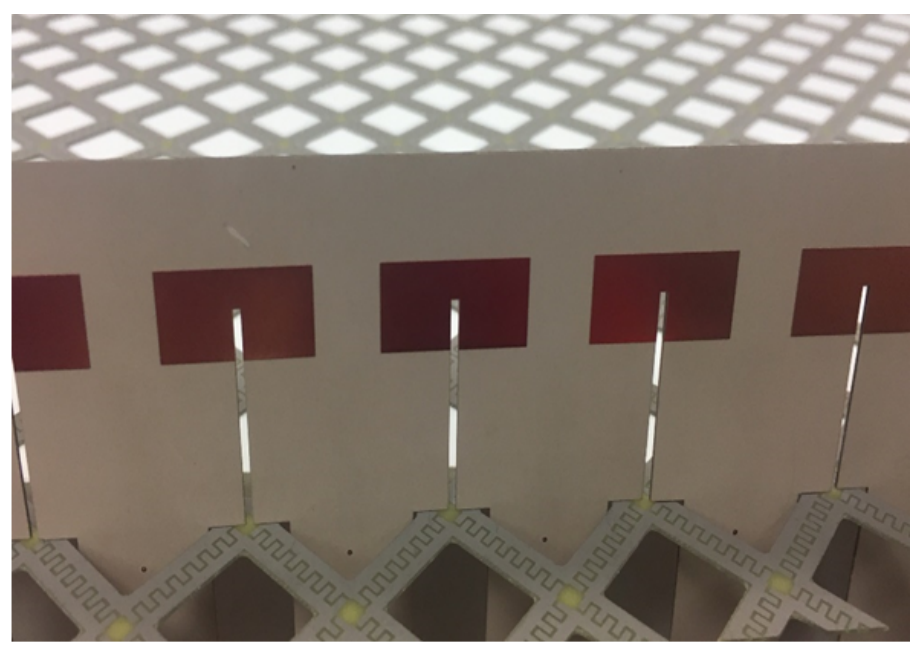

(b)

Figure 2.27: (a) Fabricated FSS Resistive Card with (b) insertion of antenna cards.

\section{Tapered Balun Fabrication}

The tapered balun feed structure was fabricated alongside the tightly coupled dipoles and metallic FSS superstrate on a two dielectric stack-up, with dimensions given in Fig. 2.28. The stripline configuration consisted of an exponentially tapered feed in the middle layer and tapered ground traces on the outer layers, for transferring the input impedance to $50 \Omega$ with complete cancellation of the common-mode currents. 
Notably, 10 mil diameter vias with optimized pitch of $2 \mathrm{~mm}$ are used to create the tapered substrate integrated waveguide ( $\mathrm{SiW}$ ) to coupled line feed transition. One dipole arm is coplanar with stripline center conductor and the second dipole arm is connected to the backside outer trace by a via as depicted at the top left of Fig. 2.28 .

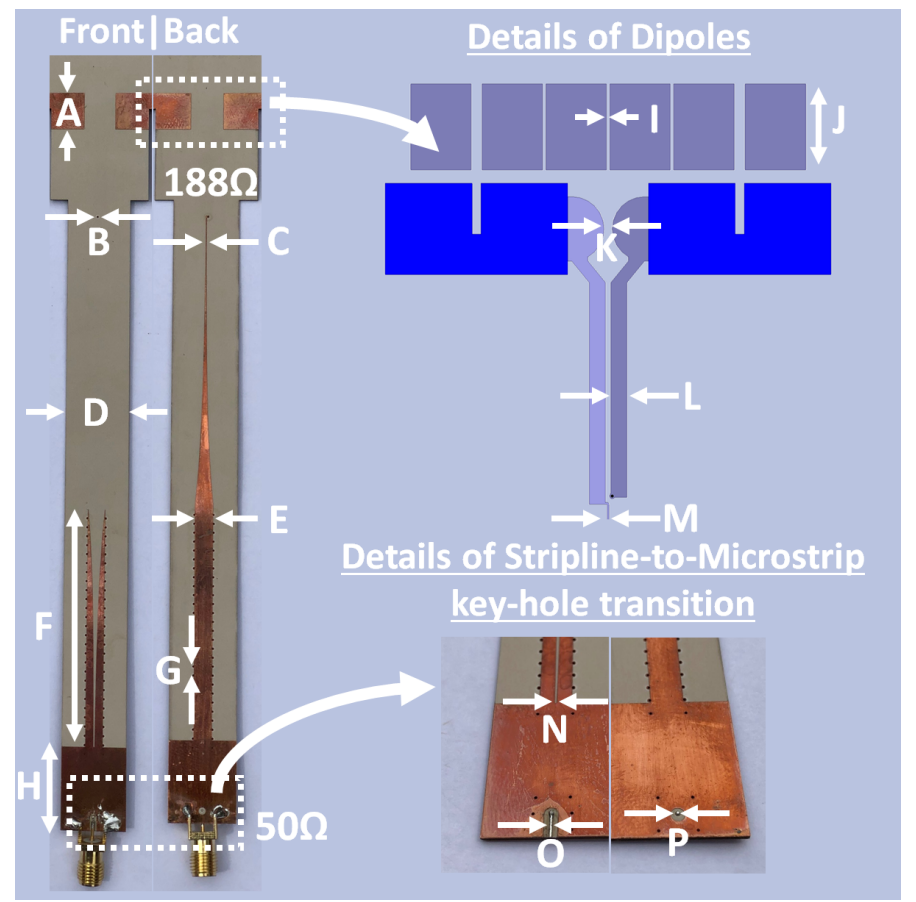

Figure 2.28: Fabricated tapered balun used to excite the tightly coupled dipoles. Note that the exponential taper in the feed line (Back) is mirrored as the tapered cut on the opposite side of the board (Front), each according to $Y(z)=$ $(D / 2) e^{-[\ln (A / D) / E]^{z}}$.

Table 2.2: Dimensions (Units : $\mathrm{mm}$ ) of tapered balun in Fig. 2.28

\begin{tabular}{|c|c|c|c|c|c|c|c|}
\hline $\mathbf{A}$ & $\mathbf{B}$ & $\mathbf{C}$ & $\mathbf{D}$ & $\mathbf{E}$ & $\mathbf{F}$ & $\mathbf{G}$ & $\mathbf{H}$ \\
\hline 8.5 & 0.254 & 0.152 & 13.62 & 4 & 52.68 & 2 & 18 \\
\hline $\mathbf{I}$ & $\mathbf{J}$ & $\mathbf{K}$ & $\mathbf{L}$ & $\mathbf{M}$ & $\mathbf{N}$ & $\mathbf{O}$ & $\mathbf{P}$ \\
\hline 0.3 & 8 & .381 & 1.5 & 0.152 & 0.152 & 0.254 & 0.254 \\
\hline
\end{tabular}




\section{Measured Results for the EWB TCDA}

The fabricated $12 \times 12$ dual-polarized array in Fig. 2.26 was measured and characterized. More in detail, we computed the active VSWR in Fig. 2.29 and Fig. ?? by adding the linear reflection coefficient of the element under test with the coupling terms from the surrounding elements of the $12 \times 12$ dual-polarized array. As seen, the measured VSWR of the central elements yields a 58:1 impedance bandwidth with VSWR $<3$ from $0.13 \mathrm{GHz}$ to $7.63 \mathrm{GHz}$ at broadside, as simulations suggested. The VSWR in Fig. 2.29 rises to a maximum of 4.4 when scanning, as expected by the $1 / \cos (\theta)$ free space impedance in the $\mathrm{H}$-plane.
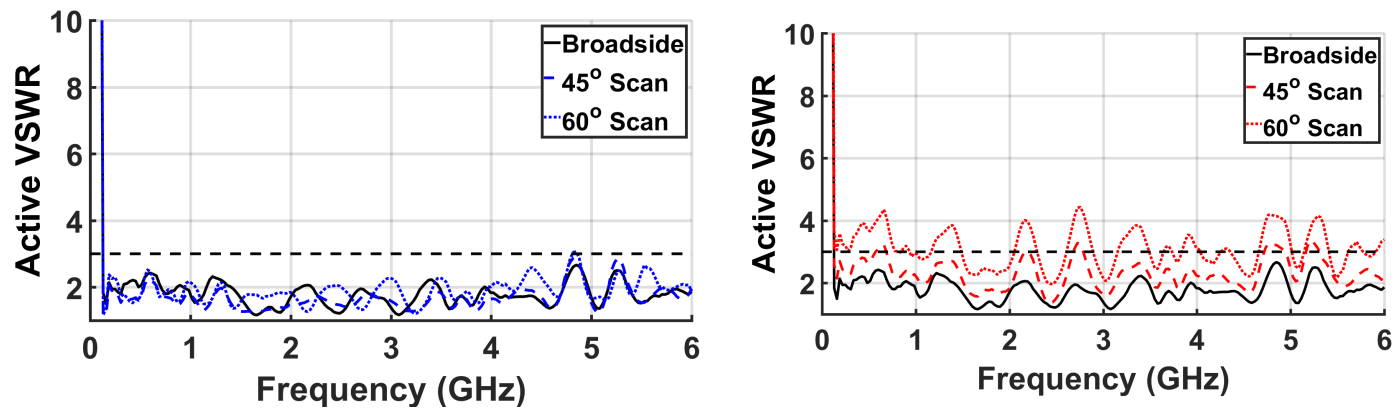

Figure 2.29: Measured E-Plane (left) and H-Plane (right) active VSWR of the center array element with scanning to $45^{\circ}$ and $60^{\circ}$ from broadside.

The measured coupling terms are presented in Fig. 2.30, with the $S_{21}$ from a center element to a collocated center element, from a center element to an element 3 unit cells away and another from the center to the edge of the array. As expected, coupling decreases with frequency and distance, with an average isolation of $22 \mathrm{~dB}$ over the band and a maximum of $11.6 \mathrm{~dB}$ for the collocated center element.

To evaluate the performance of the finite array, multiple antenna elements were measured around the fabricated prototype. As documented [Tza11], edge effects on peripheral array elements degrade low frequency performance due to a lack of mutual coupling. However, due to the nature of the resistive loading in the substrate 
these finite array effects are greatly reduced, with edge elements showing analogous VSWR and realized gain figures to the center and inner elements as depicted in Fig. 2.31 and Fig. 2.32.

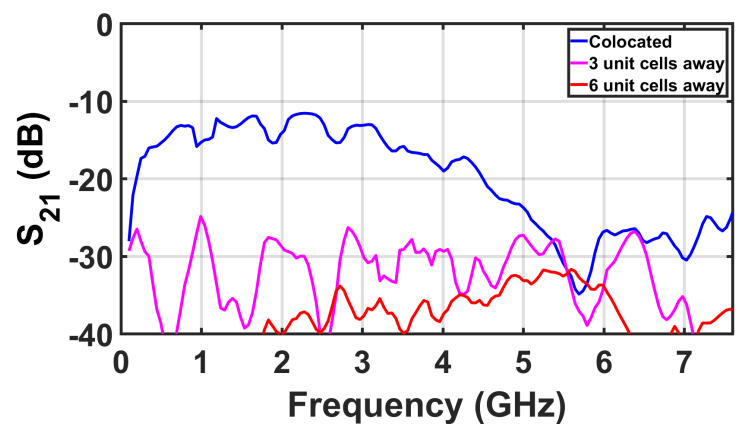

Figure 2.30: Measured coupling of the center element to other array elements at various distances.

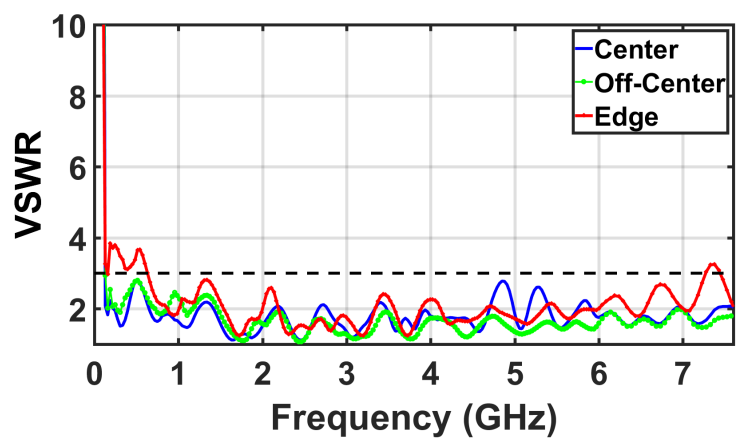

Figure 2.31: Measured broadside active VSWR of the center, off-center, and edge array elements.

The measured broadside gain of these embedded elements is plotted Fig. 2.32 with comparison to the simulations. Gain measurements are only shown for frequencies greater than $650 \mathrm{MHz}$ due to the low frequency cutoff of the reference horn. Likewise, the measured cross-polarized polarization purity is limited to 20 $\mathrm{dB}$ on average by the properties of the reference horn, with some bands showing up to $36 \mathrm{~dB}$ isolation. The measured D-plane col-polarized and cross-polarized gain according to Ludwig's 3rd definition [Lud73] are found in Fig. 2.32. As stated, the 
cross polarized gain levels are limited by the polarization purity of the reference horn used, but show more than $20 \mathrm{~dB}$ polarization purity at $\theta= \pm 0^{\circ}$. To show the cross polarization purity of the array, a D-plane $\theta= \pm 45^{\circ}$ and $\theta= \pm 60^{\circ}$ theta cut is included in Fig. 2.32 with a polarization purity of $17 \mathrm{~dB}$ on average out to $\theta= \pm 60^{\circ}$
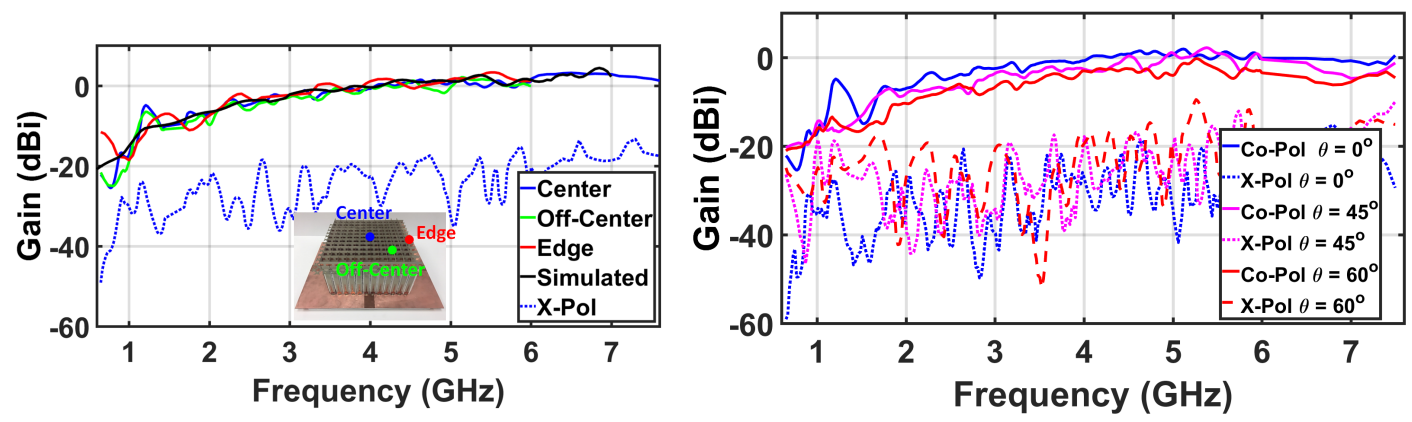

Figure 2.32: (Left)Measured broadside gain of center element vs. simulation. The co-polarized gain of several array elements is included to show finite array effects. (Right) Measured D-plane co-polarized and cross-polarized gain at theta $0^{\circ}, 45^{\circ}$, and $60^{\circ}$ cuts. A polarization purity of $17 \mathrm{~dB}$ on average is achieved out to $\theta= \pm 60^{\circ}$.

The measured gain patterns for a single center element are shown in Figs. 2.33.The patterns are extracted for scan angles of $45^{\circ}$ and $60^{\circ}$ from broadside scans using the Active Element Pattern method [Poz94] to show the scanning capability of the array with the mathematical equivalent of a loss-less beamformer up to the grating lobe frequency cutoff of $6 \mathrm{GHz}$. It can be seen that the measured patterns are well correlated with simulations for the entire band. The $7 \mathrm{GHz}$ plot in Fig. 2.33 was included to show the verification of broadside operation at the high band, with the expected grating lobes arising with the $45^{\circ}$ and $60^{\circ}$ scans. 

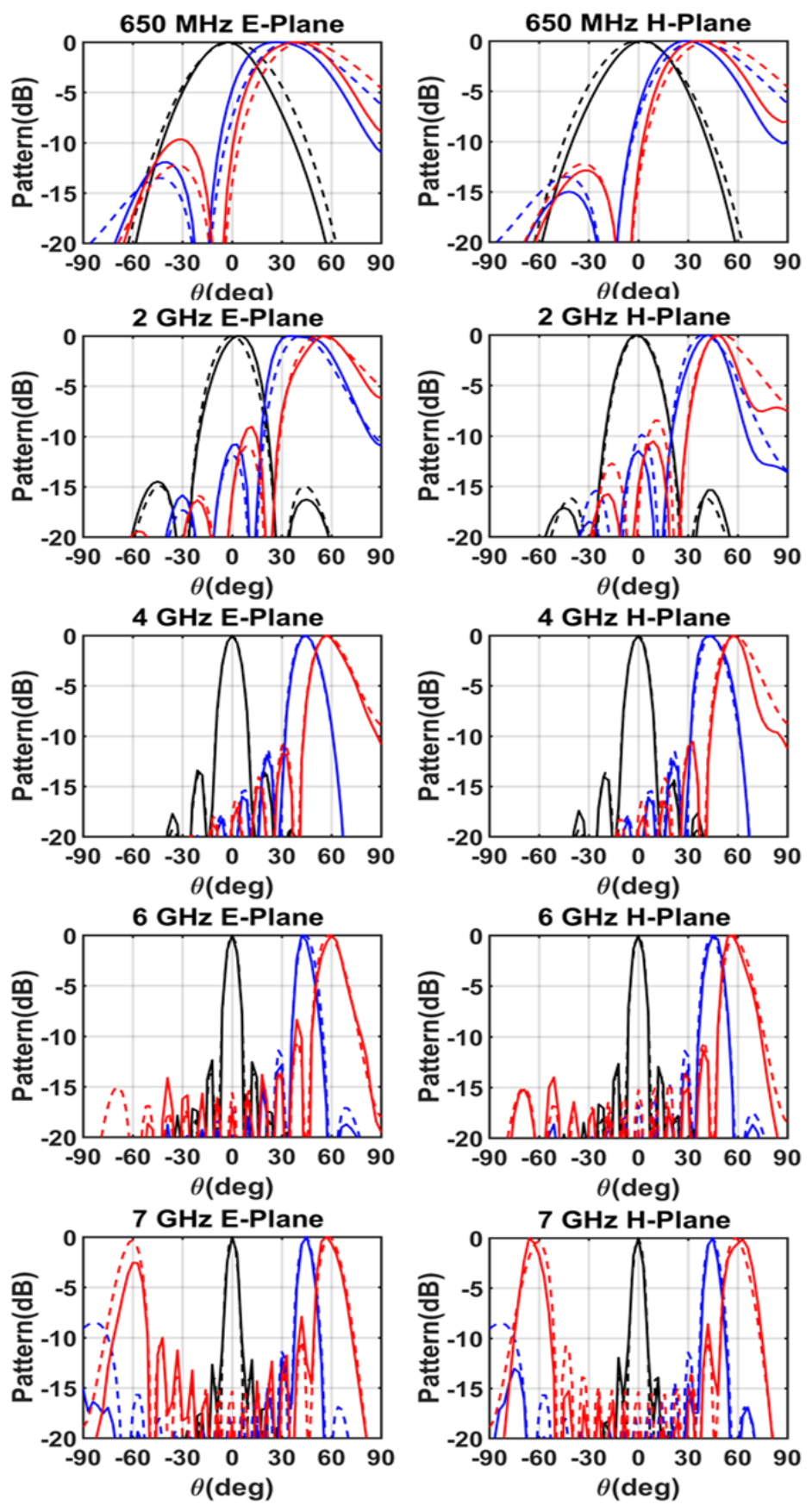

Meas Broadside -Sim Broadside- Meas $45^{\circ}-\operatorname{Sim} 45^{\circ}-$ Meas $60^{\circ}-\operatorname{Sim} 60^{\circ}$

Figure 2.33: Measured E-plane (left) and H-plane (right) gain patterns showing scanning down to $60^{\circ}$ from broadside for $650 \mathrm{MHz}, 2 \mathrm{GHz}, 4 \mathrm{GHz}, 6 \mathrm{GHz}$, and 7 $\mathrm{GHz}$ using the AEP method. 


\section{CHAPTER 3}

\section{UWB DIFFERENTIAL FEEDS}

In this chapter, TCDA with UWB differential feeding and wide-angle scanning capability are presented at UHF-S bands (0.4-3.2 GHz) and S-Ku bands (3-18 GHz). A key aspect of a differential array is its direct compatibility with existing wideband differential components [Spi16] that enable high dynamic range and linearity. Further, differential signals provide inherent lower distortion in response to noise and interference from power supplies. Even more, distortions due to even order harmonics from nonlinear devices can be suppressed by differential feeds [Poz12].

As mentioned in Chapter 2, the $\mathrm{S}-\mathrm{Ku}$ bands have great potential in airborne platforms, with coverage of altimetry, weather, surveillance, SATCOM communication, and fire control radar bands. Further, the $0.4 \mathrm{GHz}$ to $3.2 \mathrm{GHz}$ spectrum has a large number of legacy and modern applications that could easily realized with an UWB array. A TCDA that spans these frequencies could be used in a wide variety of sectors, such as: 1) the commercial band of 2.31-2.36 GHz that Sirius XM radio broadcasts on, 2) the scientific 2.29-2.3 GHz spectrum which the NASA Deep Space Network relies on, and 3) the 2.7-2.9 GHz airport surveillance bands. Even more, this UHF-S band array covers both the $900 \mathrm{MHz}$ and $2.4 \mathrm{GHz}$ industrial, scientific and medical (ISM) bands that provide a multitude of services. In fact, this applications of this spectrum are nearly endless, where one platform can seamlessly replace the narrowband Global Positioning System (GPS) systems (spread between 1.17 to 1.57 GHz), the specialized $1.42 \mathrm{GHz}$ band used for imaging hydrogen in interstellar

space, and more. Therefore the deployment of an UWB differential TCDA with scanning capability in the UHF-S bands (0.4-3.2 GHz) and S-Ku bands (3-18 GHz) is of considerable interest. 
As discussed in Section 1.5.2, all the aforementioned TCDA employed singleended feeds for low angle scanning over wide bandwidths. Unfortunately, these feeds introduce the possibility of broadside common-mode currents. Conversely, differential feeds inherently cancel common-mode currents at broadside, but exhibit common-modes when scanning to low angles. For instance, the UWB differential feeds in [BSC93, EO60, LLK04, dLAGGP ${ }^{+}$10] were plagued by common-mode resonances when scanning in the E-plane nearing angles down to $45^{\circ}$. Hence, the major challenge in the design of full differential radios is the reduction of these commonmode currents present in the feed-lines when scanning. Notably, common-modes arise for particular scan angles and frequencies in the differential feed lines due to destructive mutual coupling between elements in presence of a third conductor (i.e. ground plane). If not addressed properly, these common-modes radiate in a manner that significantly reduces total efficiency and impedance bandwidths of balanced antenna structures [S. 08]. The most prevalent way to suppress these scan-dependent common-modes is to employ a balanced-to-unbalanced feed (i.e balun), thereby avoiding differential feeding altogether [DSV13c, LKLV18, HV11]. Other methods have been explored to remove such modes by employing conductive E-Plane walls to disrupt the fields in single polarized arrays [BSC93, EO60], or by using resistive terminations to attenuate the common-modes [LLK04]-[dLAGGP $\left.{ }^{+} 10\right]$.

To realize a differential TCDA (D-TCDA), a new feed structure, referred to as the Balanced Wideband Impedance Transformer (BWIT), is presented (see Fig. 3.1 right). This feed is directly compatible with differential power amplifiers, which removes the potential phase and amplitude mismatches and associated losses of UWB baluns. Physical symmetry is used to achieve cancellation of the common-modes across a continuous $>6: 1$ impedance bandwidth while scanning. 


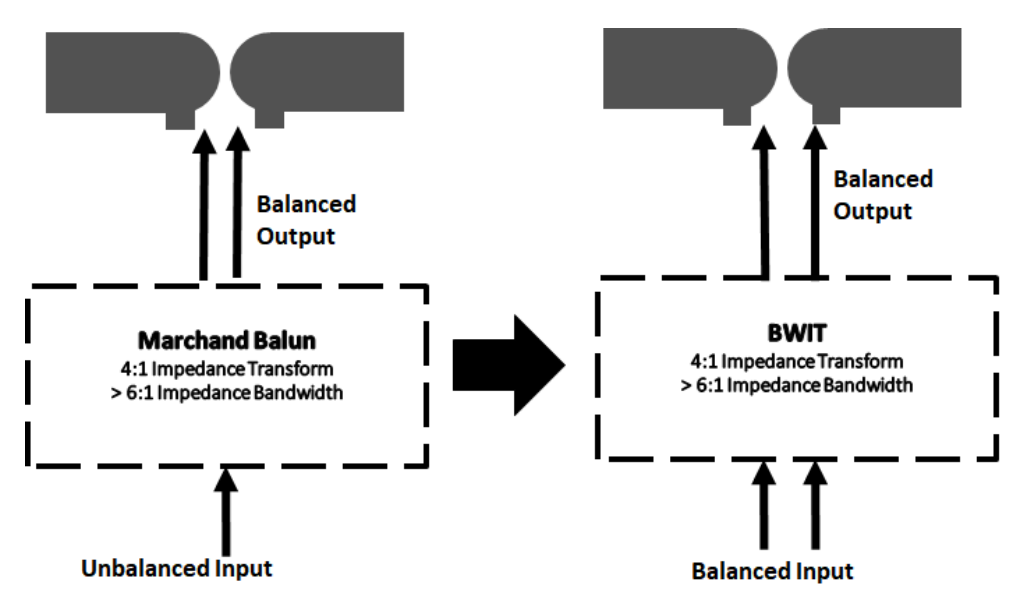

Figure 3.1: Attributes of Marchand Balun and BWIT fed tightly coupled dipoles.

\subsection{S-Band Differentially Fed TCDA Design}

\subsubsection{Differential Feeding Network}

Most previously mentioned TCDA employed an integrated Marchand balun [DSV13c] with the characteristics of that in Fig. 3.1 (left). In this chapter, the tightly coupled dipoles are being excited through the BWIT feed network of Fig. 3.2(a). This feed consists of two Marchand baluns arranged in parallel, as illustrated by the circuit in Fig. 3.2(b). As depicted, the BWIT employs a mirrored series open stub $\left(Z_{o c}\right)$ and a parallel short stub $\left(Z_{s c}\right)$ in each feed line. Symmetry between the feed lines is maintained for balanced transmission of opposite phase signals from the feed to the aperture. The length and characteristic impedance of these stubs are tuned to achieve wideband matching, with a concurrent goal to suppress common-mode currents across large bandwidths at all scan angles. Fig. 3.2(c) validates the equivalent circuit representation of the BWIT feed. While not having the exact circuit parameters, it uses tunable values to establish a behavioral model for the feed network. 
Using this equivalent circuit, the overall D-TCDA can be optimized quickly with circuit software, prior to conducting more time-consuming full-wave simulations. Fig. 3.3 shows the surface current distribution on the array, with clear cancellation of common-mode currents along the mirrored feed lines when scanning. This allows for resonance-free scanning down to $45^{\circ}$ over a wide bandwidth.

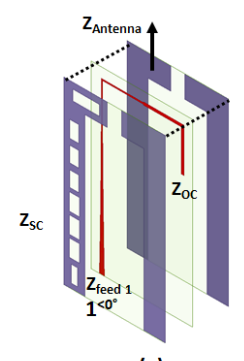

(a)

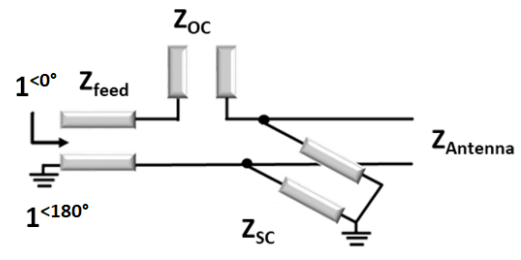

(b)

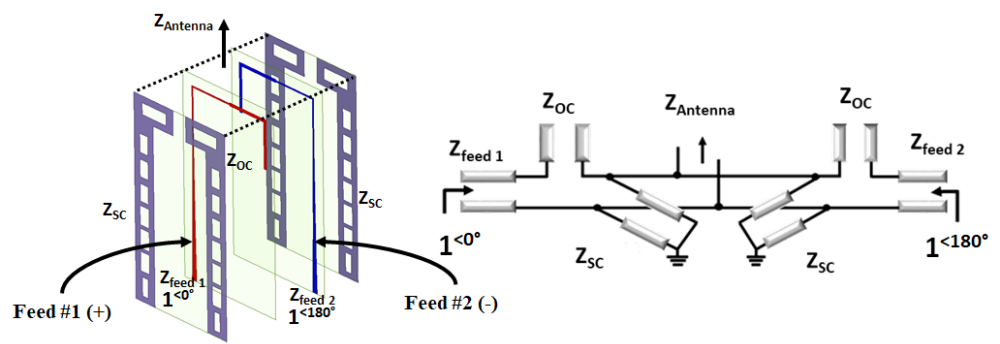

(c)

(d)

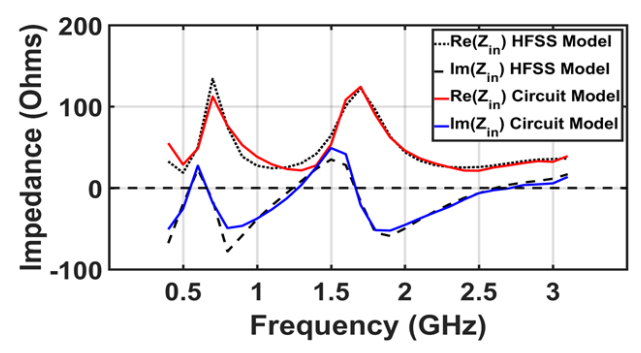

(e)

(e)

Figure 3.2: Transmission line equivalent circuits of (a) Marchand balun with (b) Equivalent Circuit and (c) Differential Feeding Network with (d) Equivalent Circuit. (e) Verification of the BWIT equivalent circuit model for the fabricated design 


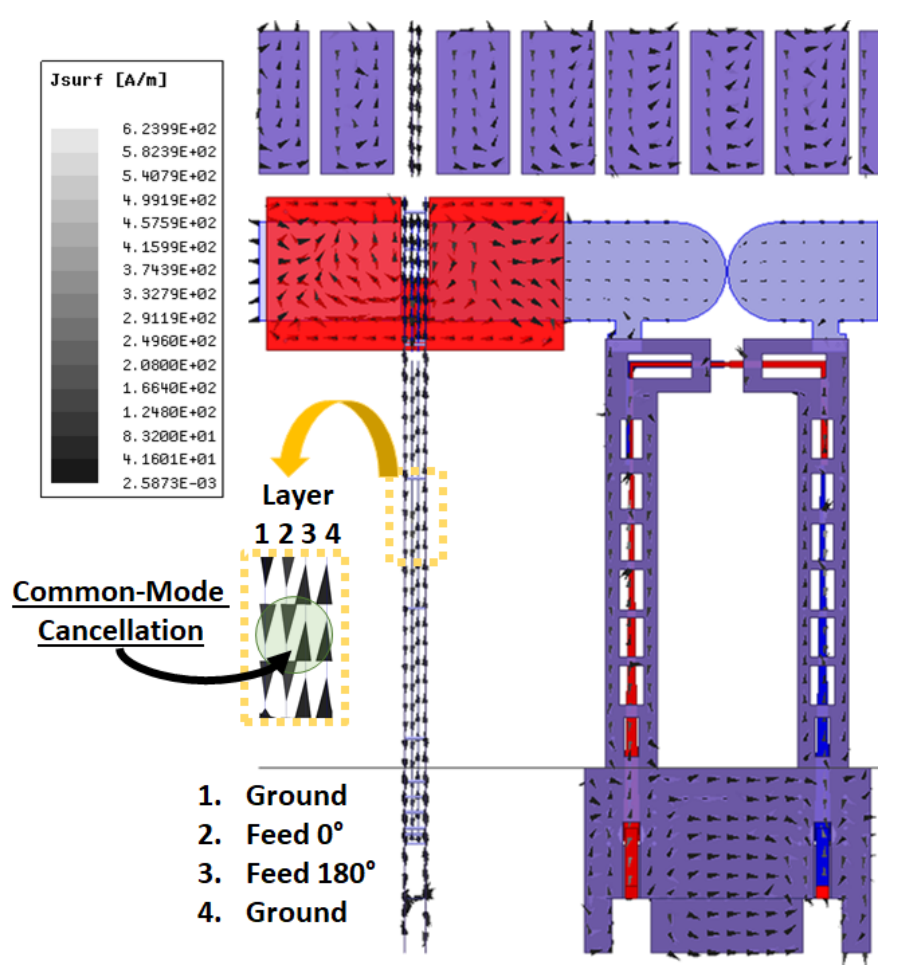

Figure 3.3: Current vectors displayed on the BWIT feed for the $45^{\circ}$ case showing common-mode current cancellation.

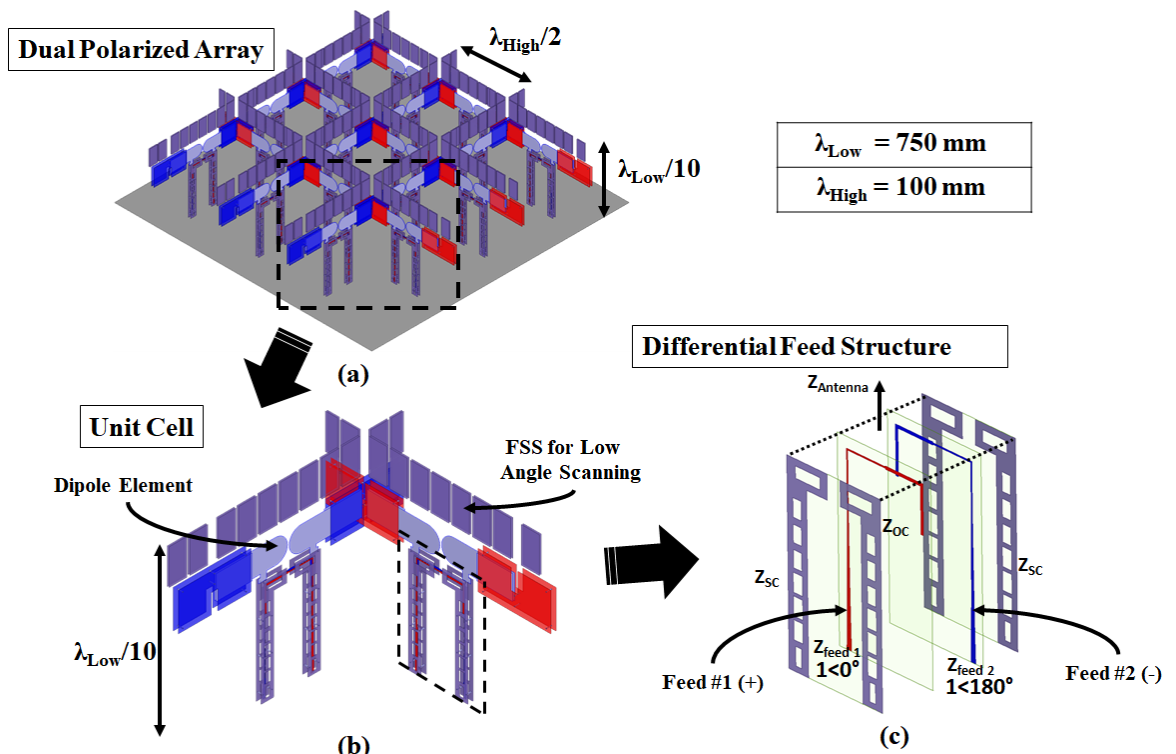

Figure 3.4: Current vectors displayed on the BWIT feed for the $45^{\circ}$ case showing common-mode current cancellation. 


\section{Tightly Coupled Dipole Element Design}

The TCDA design in Fig. 3.4 employs two independent linear polarizations using an egg-crate configuration for ease of assembly, similar to the aforementioned TCDA. Also, a metal FSS superstrate is employed for low-angle scanning with FSS design considerations following that outlined in [YGV16]. Again, the feed cards intersect at the ends of the dipoles. Fig. 3.5(a) shows the layout of a past L-band dualpolarized TCDA following the design reported in [PV16a]. For ease of fabrication, horizontal dipole arms of each linear polarization were employed. As depicted in Fig. 3.5(a), capacitive coupling among the dipoles was realized using metal strips printed on the opposite side of the substrate. Fig. 3.5(b) shows the previously described method using three-layer boards to introduce more capacitive coupling and improve bandwidth. However, the increased number of boards to realize a symmetric differential feed extends the distance between the tightly coupled dipole arms. This implies less capacitive coupling between neighboring dipoles and a smaller impedance bandwidth. To resolve this issue, a dual-offset approach to the dipole arms was investigated. This topology is depicted in Fig. 3.5(c) and compensates for the larger capacitive gaps by offsetting the dipole arms and adding co-planar parasitic coupling layers. Doing so, additional capacitance is introduced to counter the inductive effects of the ground plane and improve lower frequency performance. As depicted in Fig. 3.6, a metal via connects the dipole arms on the center layers to the feed structure on the outer layers.

\subsubsection{Differentially Fed TCDA Simulations}

For simplicity, an infinite array simulation was used to represent the $8 \times 8$ finite element array using ANSYS HFSS v.19. As depicted in Fig. 3.6, simulations were 


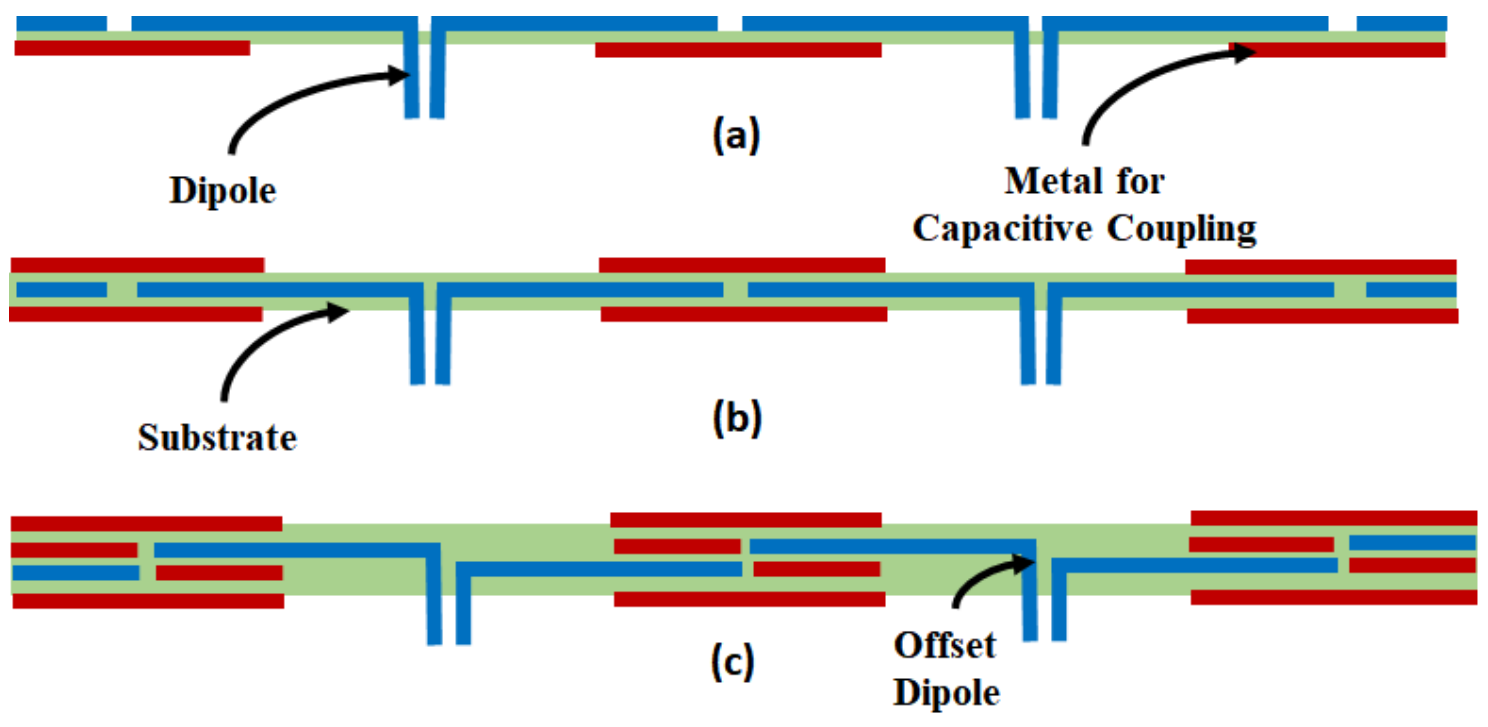

Figure 3.5: Various approaches to realize capacitive coupling in TCDAs and achieve wide bandwidths.

performed using a pair of differential $50 \Omega$ lumped ports, excited with phases of $0^{\circ}$ and $180^{\circ}$. Post-processing was used to represent the differential feed performance of each polarization as a balanced port. The infinite array VSWR in the principle $(\mathrm{E} / \mathrm{H})$ and diagonal planes (D-plane), with scanning to $45^{\circ}$ is given in Fig. 3.7. As seen, the array provides a broadside VSWR $<3.5$ across $0.4-3.2 \mathrm{GHz}$, implying an impedance bandwidth of 8:1. As typical with low profile perfect electric conductor (PEC) backed arrays, the H-plane scanning VSWR degrades at lower frequencies, given the variation in the Floquet mode impedance in both planes, with a $1 / \cos (\theta)$ term related to the resistance looking into the H-Plane [Whe64]. This can be neglected at the lower bands provided that the inherently wider beamwidths do not require wideangle scanning. Full wave gain predictions are given in Fig. 3.8 with reference to the area of a periodic unit cell. These show that a near theoretical gain is achieved with $40 \mathrm{~dB}$ average polarization isolation in the principle $(\mathrm{E} / \mathrm{H})$ planes, and $>20 \mathrm{~dB}$ in the D-plane. After optimizing the array using $8 \times \infty$ semi-finite simulations (infinite in one direction and finite in the other), the design was fabricated and measured. 


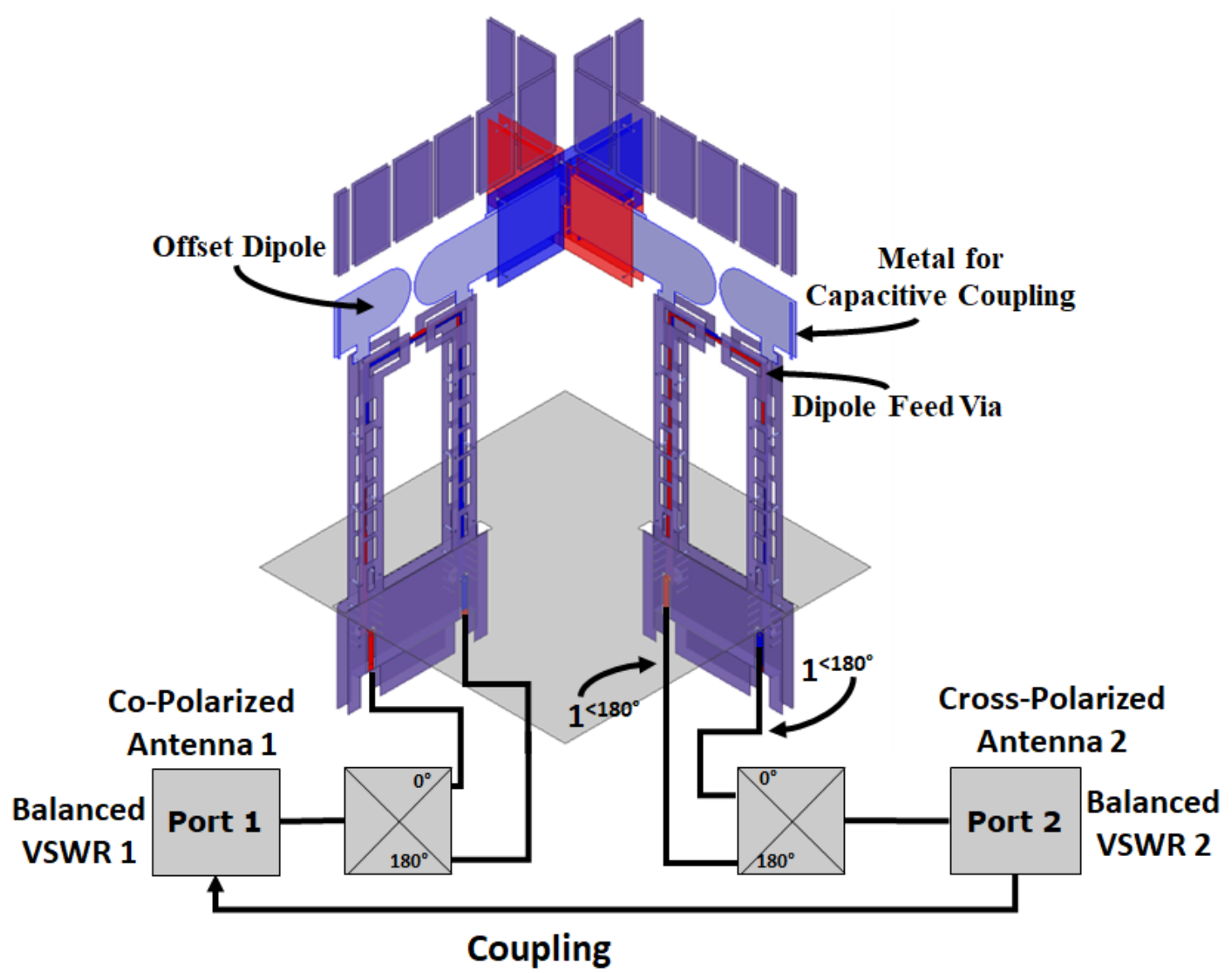

Figure 3.6: Representation of the differential feed performance for each polarization using balanced S-parameters.

\subsection{D-TCDA Prototype Fabrication and Measurements}

\subsubsection{D-TCDA Prototype Fabrication}

For validation, an $8 \times 8$ D-TCDA prototype was fabricated and measured. The antenna board was constructed from three 20 mil Rogers $5880\left(\epsilon_{r}=2.2\right)$ laminates, with a metal tolerance of $0.25 \mathrm{~mm}(10 \mathrm{mil})$ and dimensions as shown in Fig. 3.9 and Table 3.1. For the fabricated design, an FSS metal superstrate was printed on the vertical antenna cards and optimized for scanning performance, as in the previous designs. For ease of assembly, notches were cut into the dielectric boards 


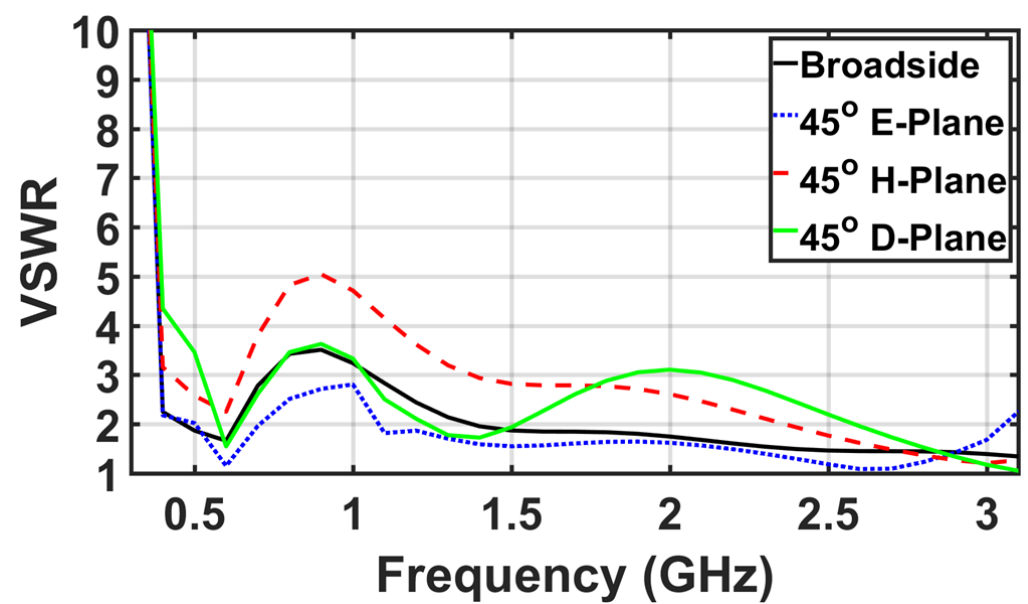

Figure 3.7: Simulated infinite array VSWR in the principle planes $(\mathrm{E} / \mathrm{H})$ of the array at broadside and scanning to $45^{\circ}$.

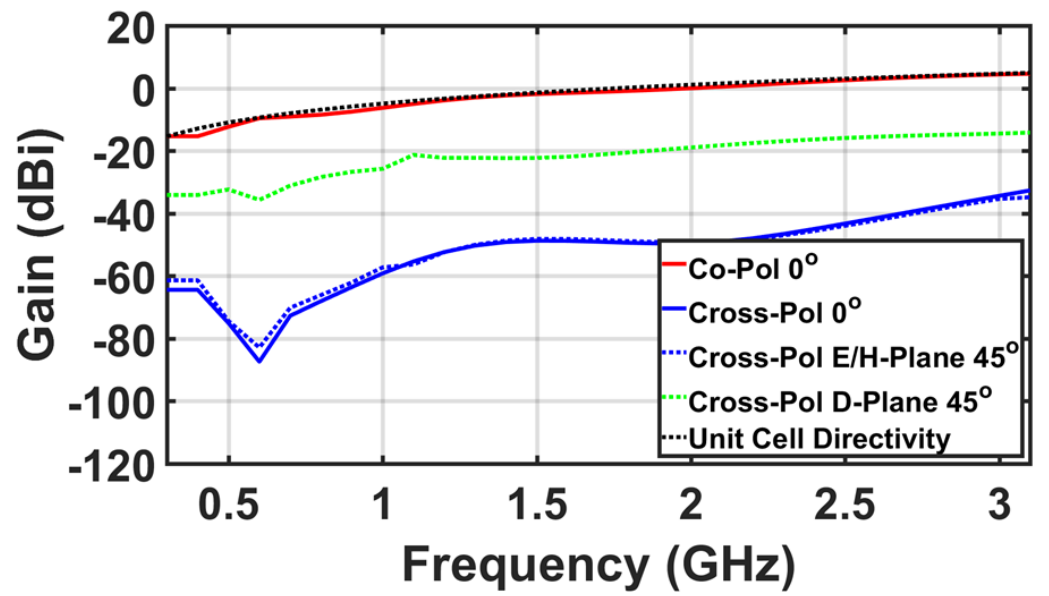

Figure 3.8: Realized Gain of the D-TCDA infinite array unit cell with $40 \mathrm{~dB}$ of polarization purity.

of the dual-polarized array to enable an egg-crate arrangement with consideration towards structural stability. Notably, no direct electrical connection or soldering was required at the joints, therefore avoiding the pitfall described in [PV16a]. A total of four ground plane sections were joined together with copper tape to form a large, lightweight, structurally stable and resonance free ground plane for testing the array (see Fig. 3.10). 


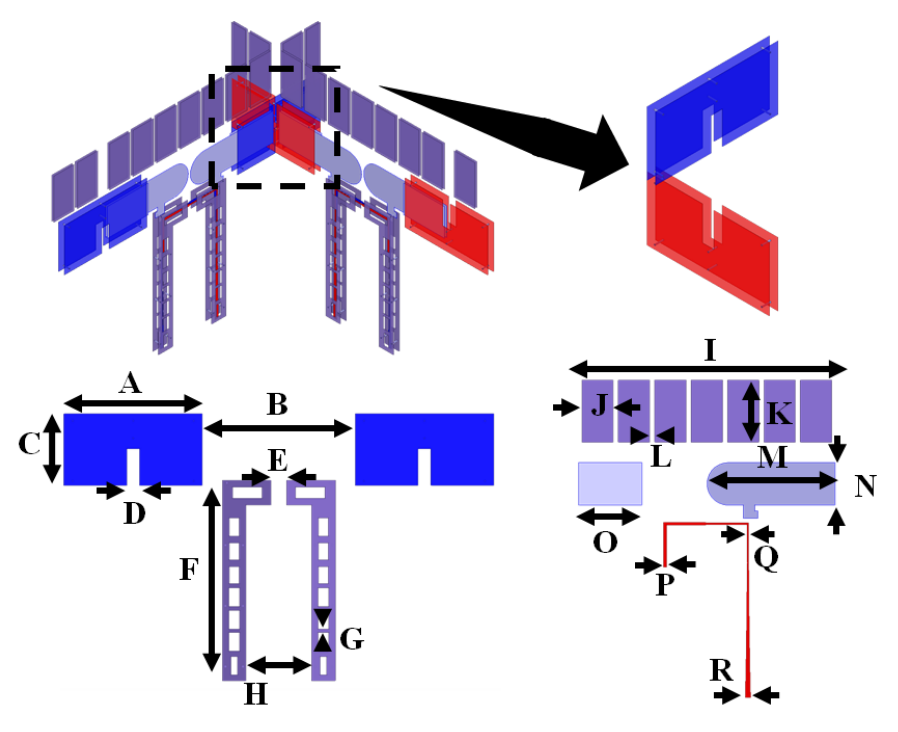

Figure 3.9: Unit cell details of the fabricated dual polarized D-TCDA. The mirrored metal layers are printed on 20 mil substrate layers with relative dialectic constant of $\varepsilon_{r}=2.2$.

Table 3.1: Dimensions (Units : $\mathrm{mm}$ ) of D-TCDA in Fig. 3.9

\begin{tabular}{|c|c|c|c|c|c|c|c|c|c|c|c|}
\hline $\mathbf{A}$ & 22.8 & $\mathbf{D}$ & 2.1 & $\mathbf{G}$ & 0.58 & $\mathbf{J}$ & 5.6 & $\mathbf{M}$ & 22.9 & $\mathbf{P}$ & 0.51 \\
\hline $\mathbf{B}$ & 25.2 & $\mathbf{E}$ & 2.6 & $\mathbf{H}$ & 10.8 & $\mathbf{K}$ & 11 & $\mathbf{N}$ & 7.6 & $\mathbf{Q}$ & 0.25 \\
\hline $\mathbf{C}$ & 11.8 & $\mathbf{F}$ & 33 & $\mathbf{I}$ & 48 & $\mathbf{L}$ & 0.91 & $\mathbf{O}$ & 11.33 & $\mathbf{R}$ & 0.91 \\
\hline
\end{tabular}

\subsubsection{D-TCDA Measurements}

For measurement, a 4-port Vector Network Analyzer (VNA) was operated in differential mode where two ports (e.g. ports 1 and 2) act as an ideal $180^{\circ}$ hybrid coupler to measure the balanced S-parameters for each dipole. Phase matched cables were used to ensure a $180^{\circ}$ phase balance between the ports. The active VSWR presented is a function of the balanced $S_{11}$ of each dipole element in addition to the balanced coupling term from neighboring dipoles (see Fig. 3.6). The embedded center and edge element gains of an otherwise match-loaded $8 \times 8$ dual-polarized array (depicted in Fig. 3.10) were measured using a Marki Microwave BAL0003 balun that was de-embedded in post-processing. 
Measured active VSWR of a center element is depicted in Fig. 3.11 as a function of frequency with comparison to semi-finite simulation results. As seen, the measured active VSWR yields an 8:1 impedance bandwidth with VSWR $<3.5$ from 0.4 GHz to $3.2 \mathrm{GHz}$ at broadside, in agreement with simulations. The measured broadside co-polarized and cross-polarized gains of an embedded center element and an edge element are plotted in Fig. 3.12 with comparison to simulations. Although normally used as a guard element for matching purposes, edge element gain is given to include finite effects, with an expected reduction in gain vs. the center element. Gain measurements are only shown for frequencies greater than $650 \mathrm{MHz}$ due to the low frequency cutoff of the reference horn. Likewise, the simulated $40 \mathrm{~dB}$ average polarization diversity is limited to $22 \mathrm{~dB}$ on average by the properties of the reference horn.

The measured gain patterns for a single center element are shown in Fig. 3.13, and are well correlated with semi-finite simulations. The patterns are extracted for scan angles of $45^{\circ}$ from broadside using the Active Element Pattern (AEP) method [Poz94]. As seen, measured patterns follow the predicted values. The measured D-plane co-polarized and cross-polarized patterns, computed in accordance with Ludwig's $3^{\text {rd }}$ definition [Lud73], are found in Fig. 3.14. As stated, the cross-polarized gain levels are limited by the polarization purity of the reference horn, but show $>20$ $\mathrm{dB}$ polarization purity down to $\theta= \pm 60^{\circ}$ from boresight. 


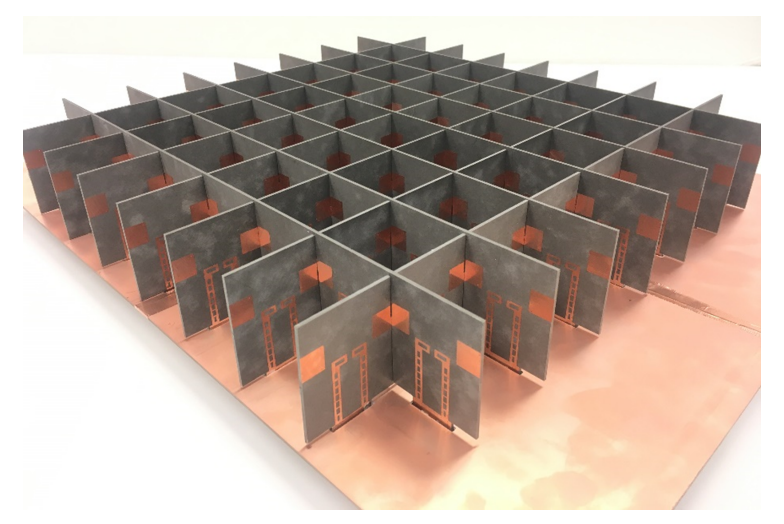

Figure 3.10: Fabricated $8 \times 8$ array.

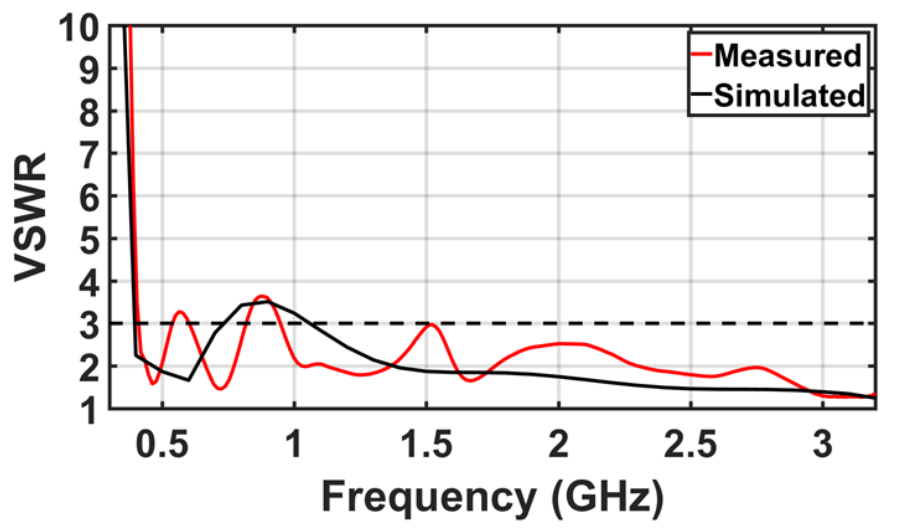

Figure 3.11: Measured active VSWR of an embedded center element as a function of frequency vs simulations.

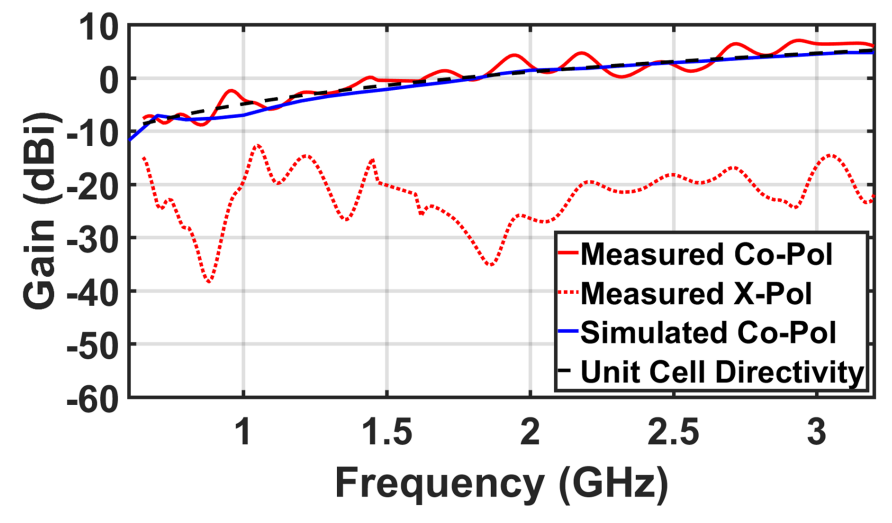

Figure 3.12: Measured broadside gain of an embedded center element as a function of frequency vs simulations. A polarization purity $>20 \mathrm{~dB}$ is achieved across most of the band 

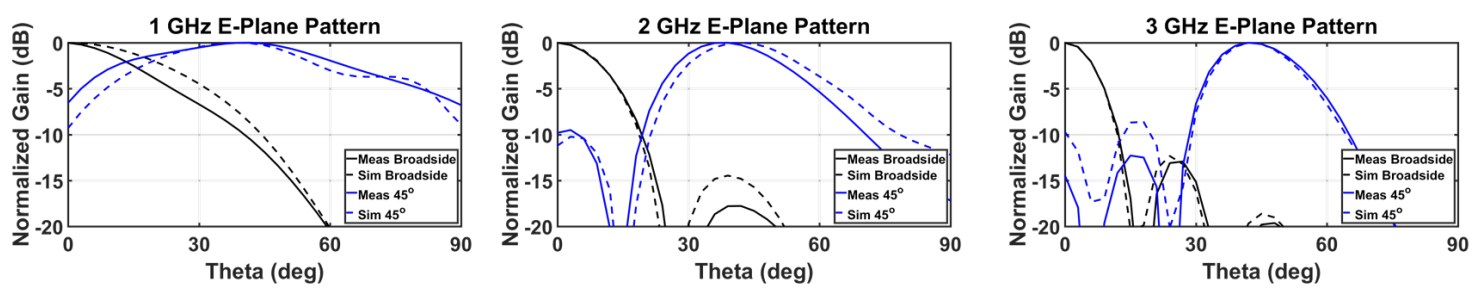

(a)
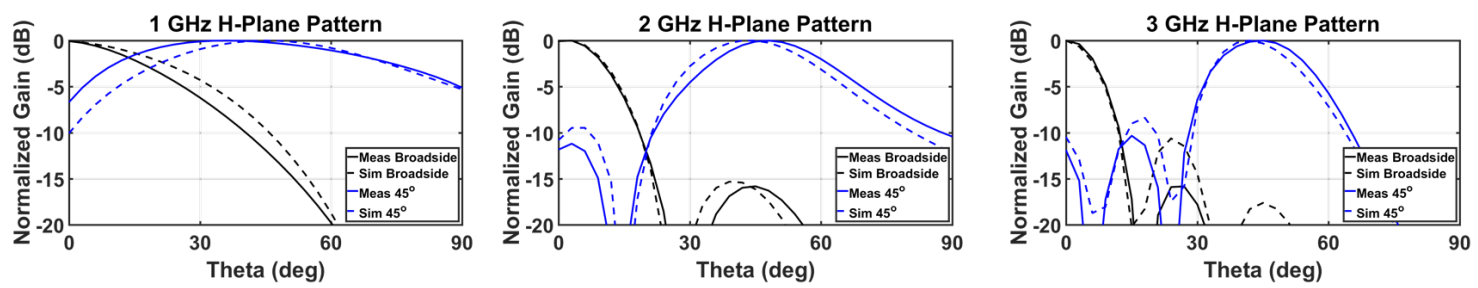

(b)

Figure 3.13: Measured center element (a) E-Plane and (b) H-plane patterns vs simulations at $1 \mathrm{GHz}, 2 \mathrm{GHz}$, and $3 \mathrm{GHz}$.
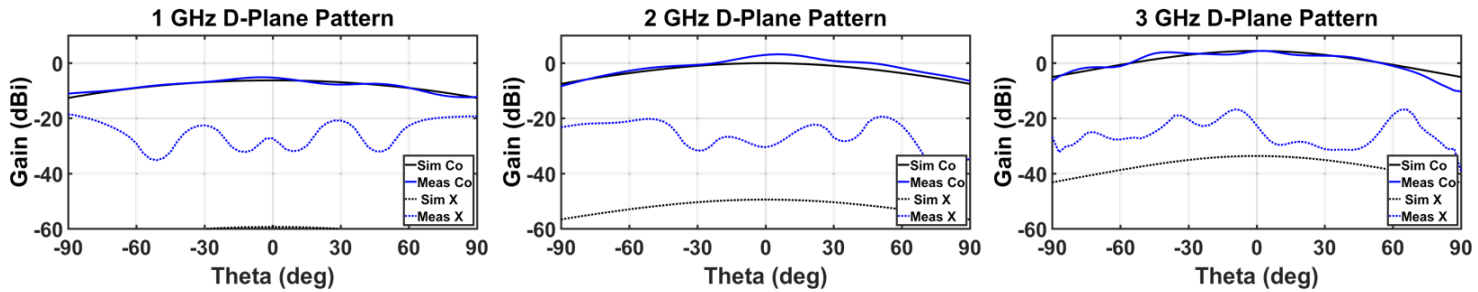

Figure 3.14: D-plane patterns vs simulations at $1 \mathrm{GHz}, 2 \mathrm{GHz}$, and $3 \mathrm{GHz}$. 


\subsection{Ku-Band Differentially Fed TCDA Design}

\subsubsection{Ku-Band Differentially Fed TCDA Simulations}

To show the frequency scalability of the BWIT feed structure, the array was scaled to Ku band operation with $18 \mathrm{GHz} \lambda / 2$ spacing. Of course, direct frequency scaling shows a 1:1 frequency performance shift, but this is not always viable due to the set thicknesses of PCB substrates. For this array, depicted in Fig. 3.15, the scaled D-TCDA was designed on three layers of 5 mil thick rigid Isola Tachyon $\left(\epsilon_{r}=3.0\right)$ substrates to ensure mechanical stability for the small array.

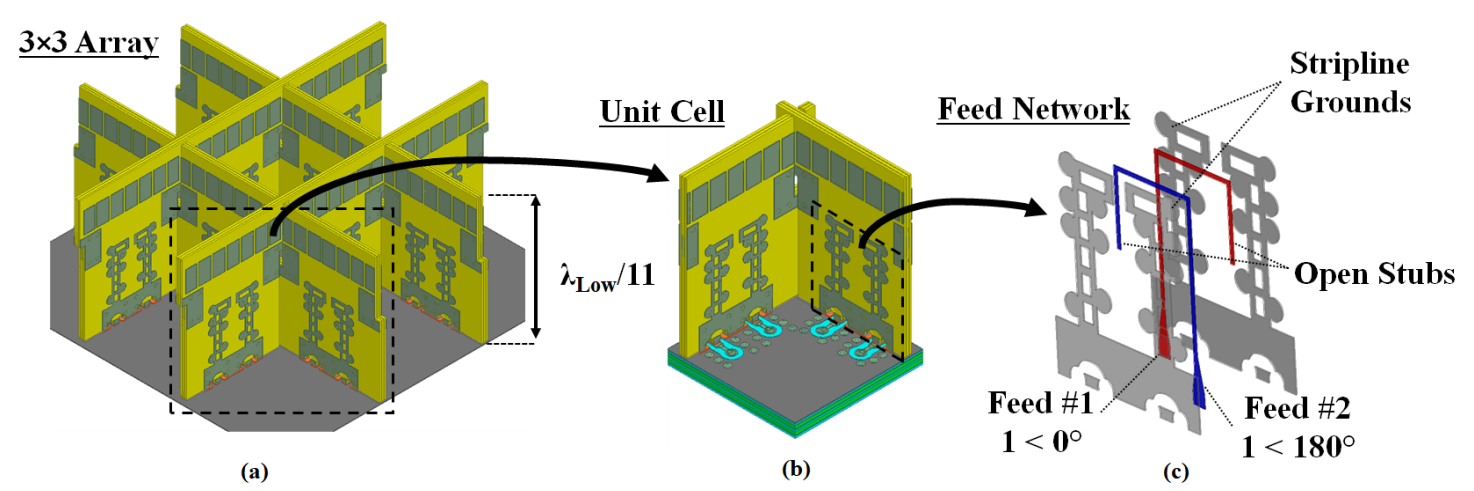

Figure 3.15: Pictorial representation of (a) Ku band D-TCDA array, (b) unit cell with planar breakout feed structure, and (c) BWIT details

The Ku band array was designed using standard PCB tolerances of $0.15 \mathrm{~mm}$ (6 mil), with feature sizes as in Fig. 3.16. The simulated performance of this scaled version is found in Fig. 3.17, with 6.4:1 impedance bandwidth with VSWR $<3$ across most of the band. Again, the H-plane scanning VSWR degrades at the lower frequencies, given the Floquet mode impedance $1 / \cos (\theta)$ behavior in the $\mathrm{H}$ Plane [Whe64]. As expected, a near theoretical gain is predicted for the matched bandwidth, with more than $70 \mathrm{~dB}$ of polarization isolation in the principle planes. 


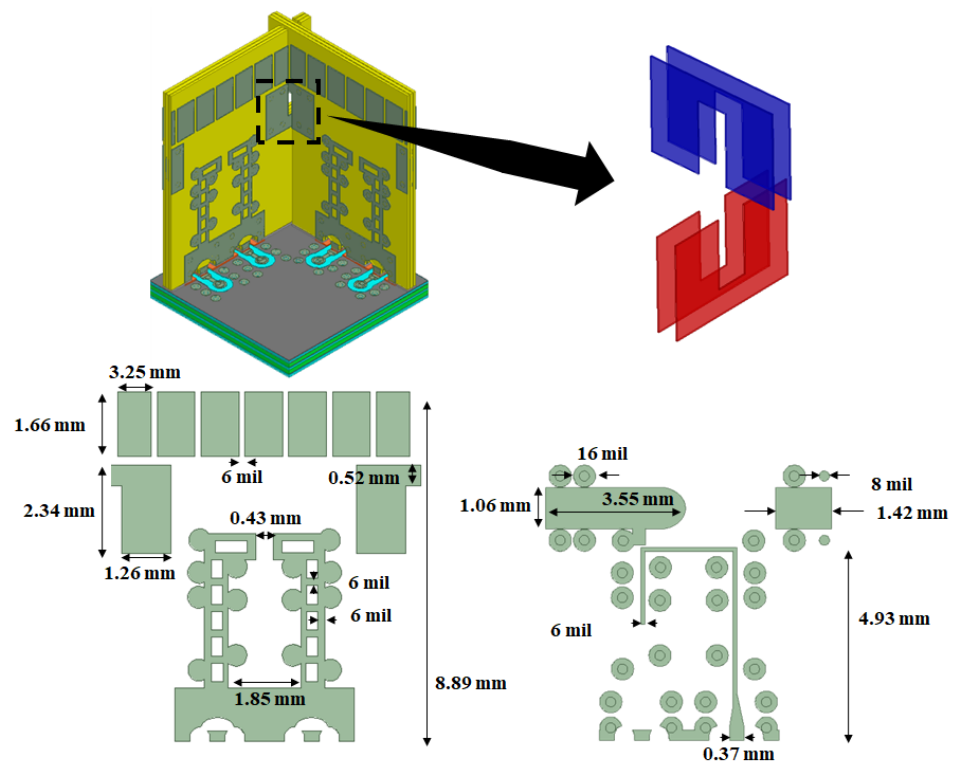

Figure 3.16: Design details of Ku band D-TCDA antenna cards

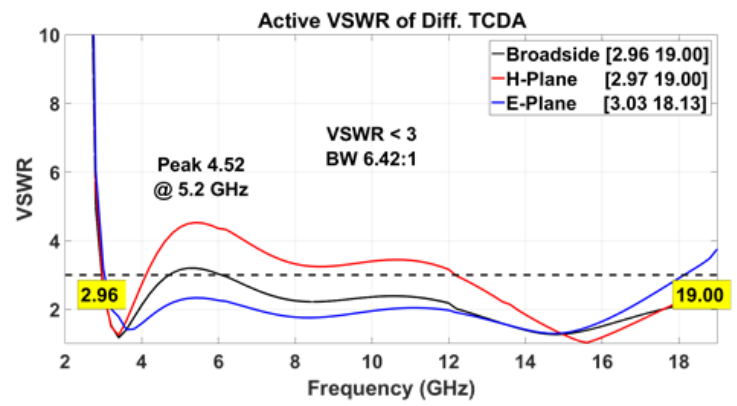

(a)

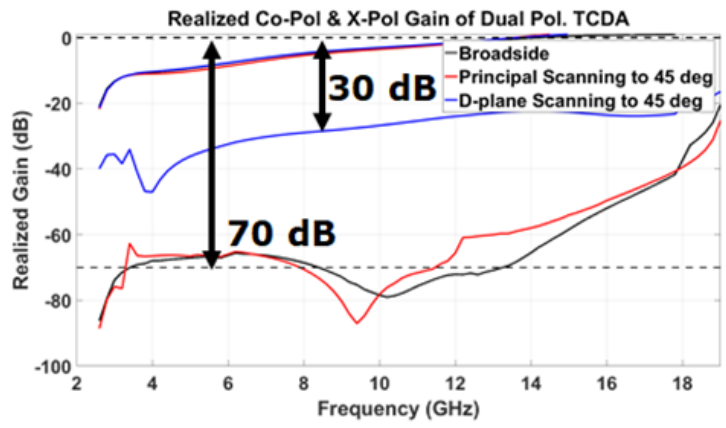

(b)

Figure 3.17: (a) Active VSWR and (b) broadside gain of Ku band D-TCDA infinite array simulation. 


\subsubsection{Planar Feed plan}

It is widely known that fabrication of a high frequency array with small tolerances is challenging. At Ku-band, the tolerances of PCB manufacturing must be carefully considered in the design process, with metal thickness and via pad diameter resulting in a significant change to the ideal design. Furthermore, at high frequencies, direct connection between the antenna card and connectors becomes difficult, especially in the case of a dual-polarized differential unit cell. At these frequencies, even SMPM and G4PO connectors are prohibitively large in size to actively feed multiple differential elements. Hence, the vertical antenna cards were designed to be edgeplated and drilled, for mounting to a breakout board to embedded stripline signal paths. The breakout board in Fig. 3.18 was designed to integrate into a fully printed system on PCB. This test structure can be de-embedded via post-processing to characterize the antenna in absence of the feed line losses.

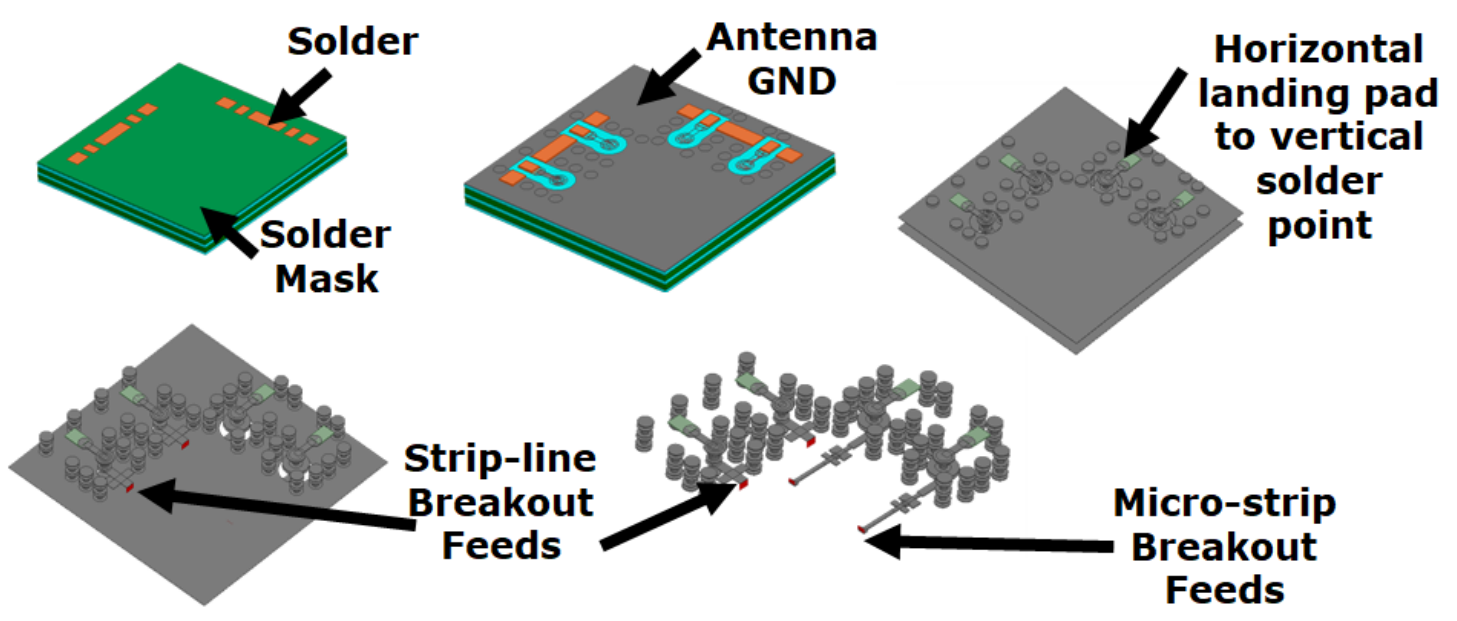

Figure 3.18: Feed board for $18 \mathrm{GHz}$ D-TCDA. 


\section{CHAPTER 4 \\ STUDY OF LOW-COST MM-WAVE TCDA}

With rapidly increasing demand for high data rates, wirelessly connected devices (e.g. cell phones, cars, watches) need multi-functional radios at millimeter-wave (mm-wave) and 5G frequencies. To accommodate these sparsely allocated spectrums (e.g. $6 \mathrm{GHz}, 28 \mathrm{GHz}, 60 \mathrm{GHz}, 77 \mathrm{GHz}$ ), antennas must be ultra-wideband (UWB) and integrated with transceivers. Further, the high path-losses at these frequencies require high gain arrays with wide-angle spatial coverage. At present, many of available $\mathrm{mmW}$ arrays are fabricated using expensive micro-fabrication techniques such as integrated antennas-on-chip [SJL $\left.{ }^{+} 14, \mathrm{SCNS18}\right]$ and Low Temperature Co-

fired Ceramic (LTCC) [LLJ+19, LCQ14]. Of course, integrated antennas-on-chip antennas have the draws of compact profiles and ease of integration with RF integrated circuits (RFIC), but are plagued by the losses of the Silicon substrates. The resulting low efficiency can be improved through the use of FSS and EBG structures, but only over a narrowband $\left[\mathrm{BAP}^{+} 12, \mathrm{AJM}^{+} 17\right]$. Conversely, Antenna-in-Package (AiP) arrays employ low loss substrates (i.e. LTCC, PCB) for improved radiation efficiency. Yet LTCC in practice involves shrinkage factors and is known for low processing yields $\left[\mathrm{KLN}^{+} 11\right]$. Notably, both the Si substrates and LTCC tapes have high dielectric constants $\left(\epsilon_{r}>6\right)$, which can introduce surface waves and preclude phased array operation. Further, these LTCC and micro-fabrication techniques are very expensive.

Recent UWB arrays have addressed the expensive fabrication problem with flipchip printed circuit board (PCB) antenna fabrication in mind [JDRGHN $\left.{ }^{+} 18\right]$. However, the majority of the focus on $5 \mathrm{G}$ has been on the narrowband implementation of the $60 \mathrm{GHz}$ ISM bands $\left[\mathrm{ACP}^{+} 13\right.$, HBG13]. Past works have introduced the idea for using PCB for UWB mm-wave antennas, but without plans for integration into 
5G radios [Nov17, MKCS17, WFQX17]. Further, these arrays lack the polarization diversity and differential feeding necessary for $5 \mathrm{G}$ applications [Spi16]. The following dual-polarized TCDAs will serve as the basis for testing and evaluating future 5G beamformers operating from $\mathrm{C}$ to $\mathrm{W}$ bands and beyond. Notably, these array are designed on PCB substrates for peak radiation efficiency, to be later integrated into a System in Package $(\mathrm{SiP})$ radio. These arrays enable the realization of future 5G UWB beamformers and transceivers.

\subsection{Novel Mm-wave Beamformer Architecture}

Realizing low cost fabrication and beamforming across wide bandwidths in mmwaves is the foundational challenge of this chapter. To address this challenge, we present multiple mm-wave TCDA as the physical layer of the envisioned $5 \mathrm{G}$ radio in

Fig. 4.1. As described in prior chapters, one design variation employs a frequency scaled Marchand balun for single ended receiver applications, with another architecture employing differential feeds. Our novel designs brings forward the following innovations:

1. Dual-linear polarized and ultra-wideband (UWB) scalable aperture TCDAs operating across $>50 \mathrm{GHz}$ of instantaneous bandwidth. These designs realize full polarization diversity for reliable communications.

2. Array thickness is $\left(<\lambda_{H i g h} / 2\right)$. Such small dimensions make these arrays very attractive for mobile platforms.

3. Wide angle beam-steering/scanning capabilities down to $60^{\circ}$ from broadside to enable wider spatial communication among small mobile platforms.

4. Multiple UWB feeding techniques with vertical integration for reliable operation and low-cost integration of mm-wave phased arrays. 


\subsubsection{Multi-Layer Antenna Integration}

Each array is designed for layer-by-layer integration to mate directly with other modules onto a core signal distribution panel. This follows the SiP approach that incorporates 1) PCB array plane, 2) RF front-end circuitry (e.g. flip-chip SiGe die), and the 3) digital modules packaged ball grid array (BGA). Details of the envisioned integration are given in Fig. 4.1. The two most obvious advantages are compactness and mobility. Further, this hybrid packaging approach allows for co-integration of custom and commercial off-the-shelf (COTS) components using standard fabrication processes. The fabricated mm-wave arrays will directly integrate with RFIC transceivers for high efficiency millimeter-wave flip-chip radios through vertical electrical interconnects (e.g. BGA). Notably, the ground plane doubles as a protective layer to isolate the efficient PCB radiators from lossy RFIC back-end components. However, in this dissertation, the array is first vertically interconnected to a UWB feed board and tested in standalone fashion for validation of the aperture layer.

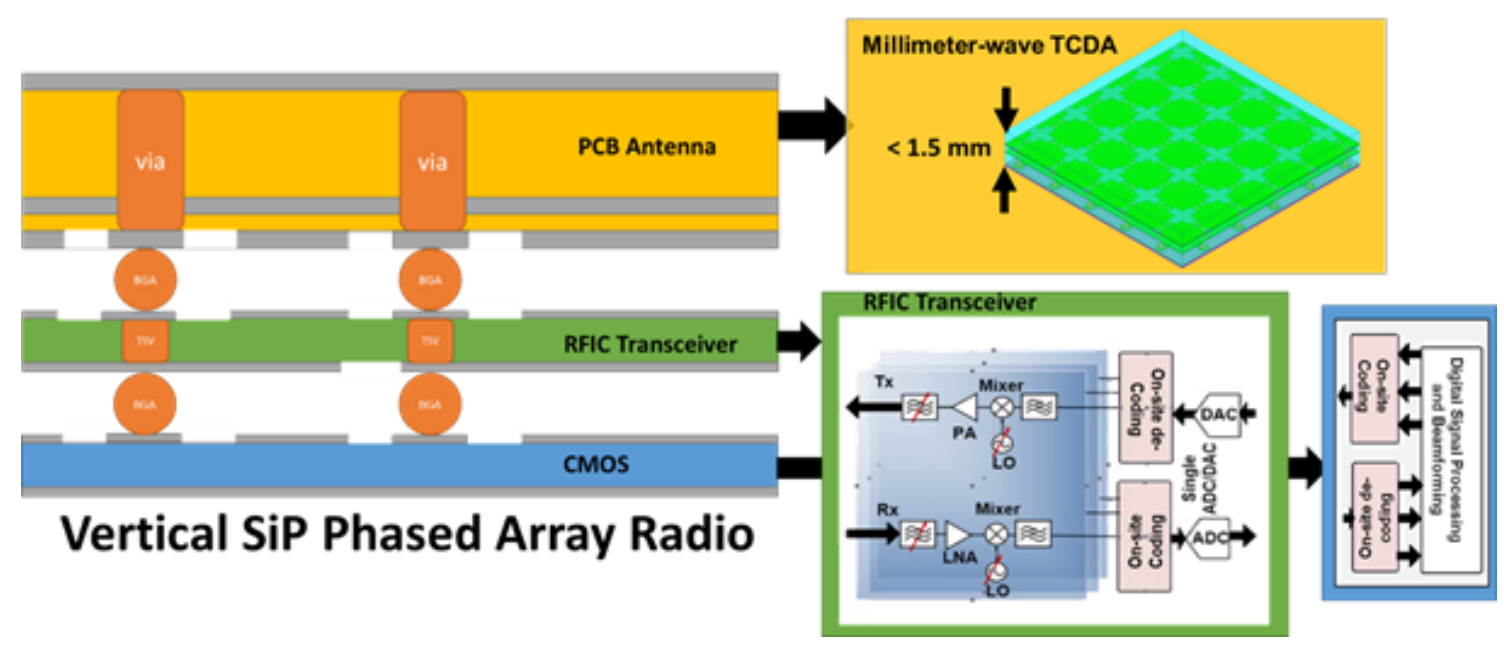

Figure 4.1: Integrated millimeter-wave transceiver concept. 


\subsection{Egg-Crate Design with Simplified Balun Feed}

\subsubsection{Simplified Marchand Balun for $30 \mathrm{GHz}$ Operation}

The array in Fig. 4.2 employs two polarizations of tightly coupled dipoles excited by a simplified microstrip Marchand Balun feed. This feed draws from Chapter 2 with an integrated Marchand Balun that acts as a higher order matching network. Again, the Marchand Balun uses a series open stub and a parallel short stub, whose impedance and lengths are tuned to achieve a broadband match. Contrary to the antenna in Chapter 2, these designs use only a single substrate layer (viz. microstrip instead of stripline), for extremely low cost fabrication. Further, no vias are included in the balun design, for further simplification. This microstrip feed efficiently transfers the $50 \Omega$ port impedance to the $188 \Omega$ input impedance of the tightly coupled dipole for operation from $4 \mathrm{GHz}$ to $30 \mathrm{GHz}$. The infinite array VSWR for this array (see Fig. 4.2) is given in Fig. 4.3.

As seen, the array provides a broadside VSWR $<3$ across $4 \mathrm{GHz}$ to $30 \mathrm{GHz}$, implying a broadside impedance bandwidth of 7.5:1. Notably, this array scans down to $60^{\circ}$ in all planes with a VSWR $<4$ across the band. The simulated gain of a unit cell is provided in Fig. 4.4 for the E/H and D-planes with a close comparison to the theoretical gain. Also depicted in Fig. 4.4, the E/H-plane cross-pol isolation exceeds $20 \mathrm{~dB}$ across all scan angles, and the D-plane shows more than $10 \mathrm{~dB}$ while scanning to $60^{\circ}$. Scan patterns for $5 \mathrm{G}(6 \mathrm{GHz}$ and $28 \mathrm{GHz})$ and SATCOMM (18 $\mathrm{GHz}$ ) frequencies are given in Fig. 4.5. As expected, the patterns for the derived Active Element Pattern (AEP) [Poz94] are similar to the simulated semi-finite case. These patterns show that $60^{\circ}$ scanning is achieved for frequencies of commercial and scientific interest. 


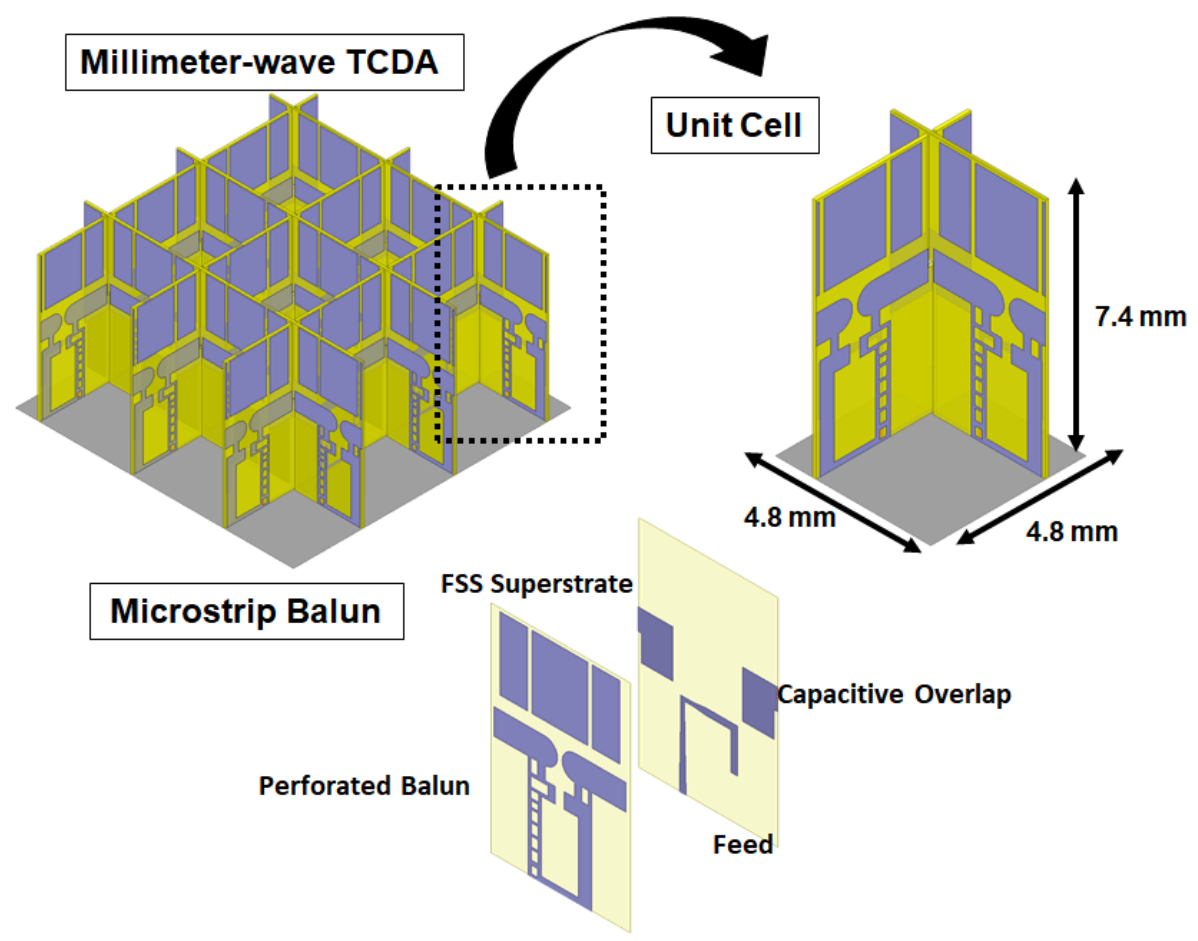

Figure 4.2: Pictorial representation of Ka-band array, unit cell, and feed details.

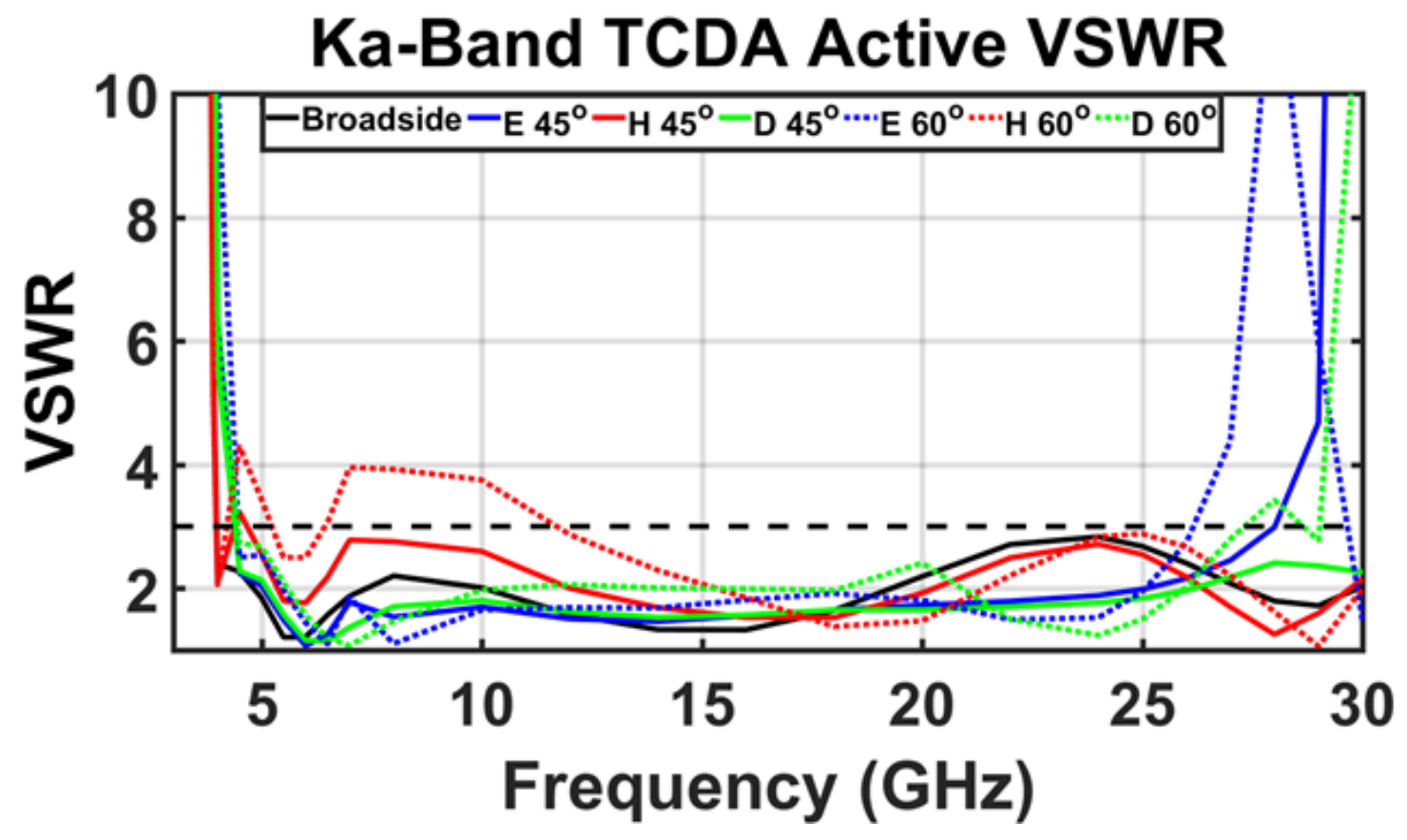

Figure 4.3: Simulated active VSWR of Ka-band TCDA infinite array unit cell 

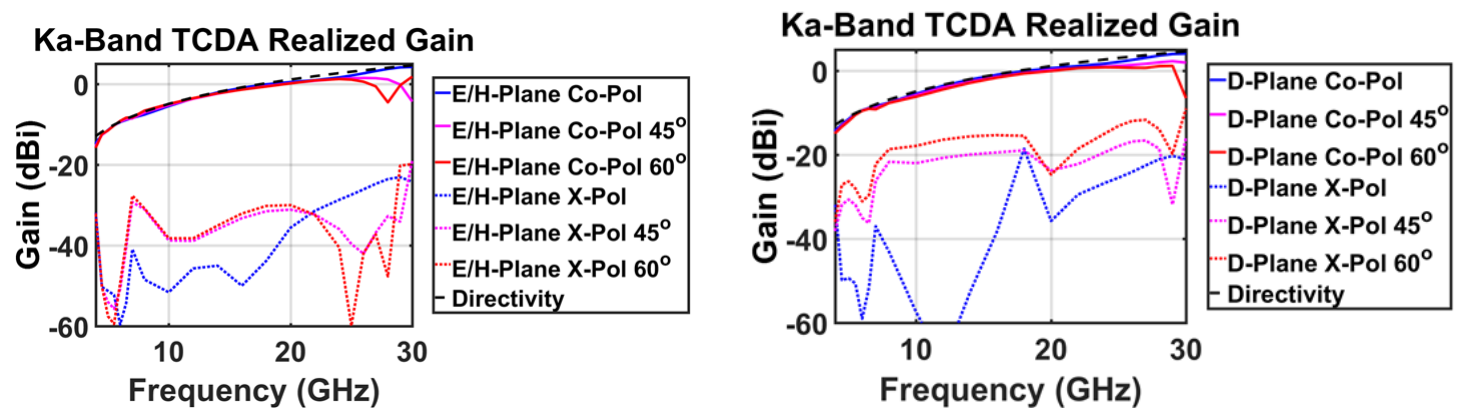

Figure 4.4: Realized gain in the principle planes $(\mathrm{E} / \mathrm{H})$ (left) and D-plane (right) for the simulated unit cell with scanning down to $60^{\circ}$ from broadside.
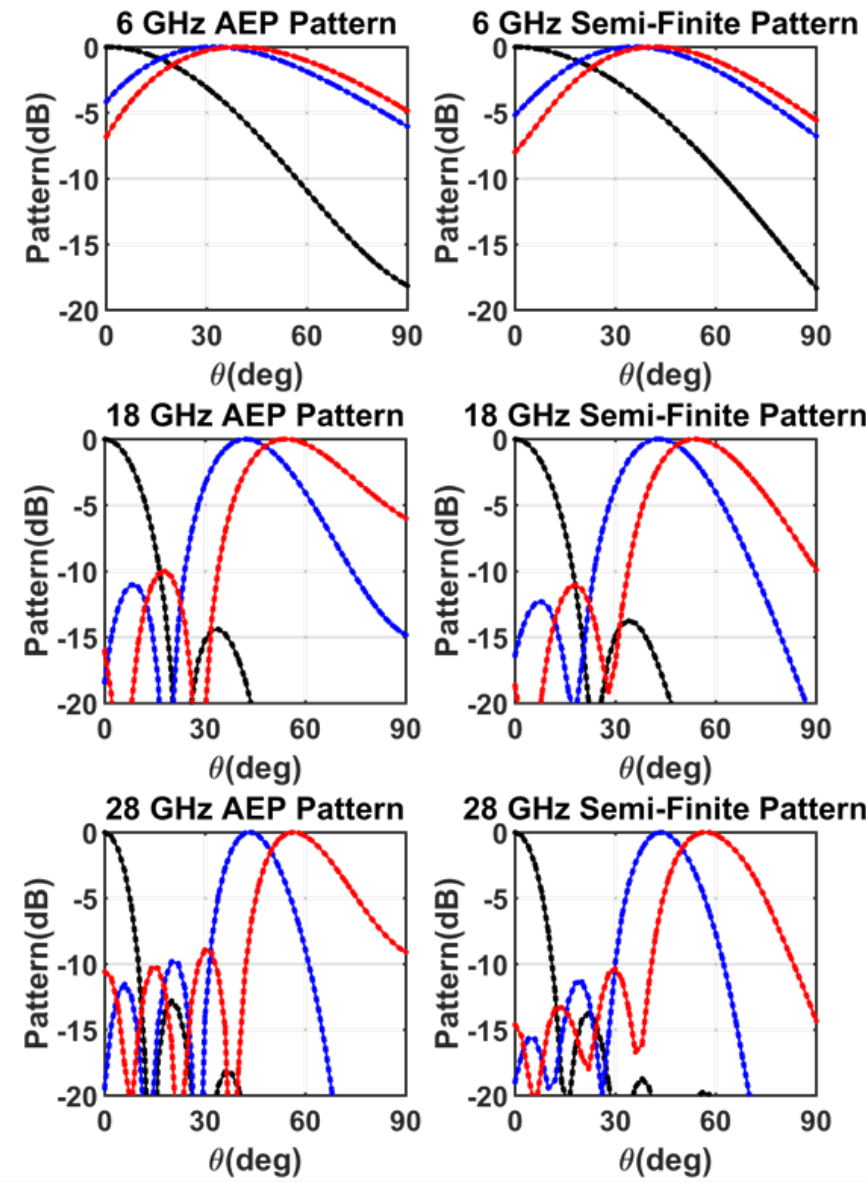

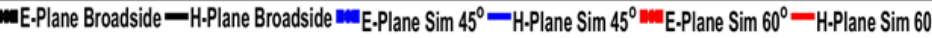

Figure 4.5: Realized gain patterns of Ka-band TCDA using AEP (left) and finite array simulations (right) with scanning to $\theta=0^{\circ}, 45^{\circ}$, and $60^{\circ}$. 


\section{Ka-Band Egg-Crate mm-Wave TCDA Fabrication}

The low-cost array employs standard PCB processes and dimensions for massproduction. The antenna was constructed from one rigid Rogers $4003\left(\epsilon_{r}=3.6\right)$

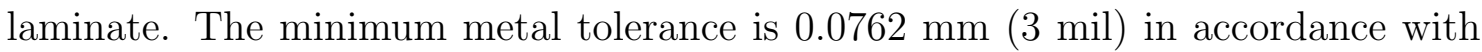
commercial PCB processes, as detailed in Fig. 4.6 and Table 4.1. As depicted, the 76.2 micron metal traces were accurately printed using the LPKF Protolaser U4 laser etcher at FIU's RFCOM Lab. As with previous TCDA, the Ka-band array is constructed in an egg-crate, as depicted in Fig. 4.7 (left). This UWB integrated array in Fig. 4.7 (right) is an attractive candidate for mobile SATCOM and 5G platforms due to its small size and weight $(<8 \mathrm{~mm}$ tall and $<1 \mathrm{~g})$. The UWB feed structure used to excite the array is discussed in the following sections.
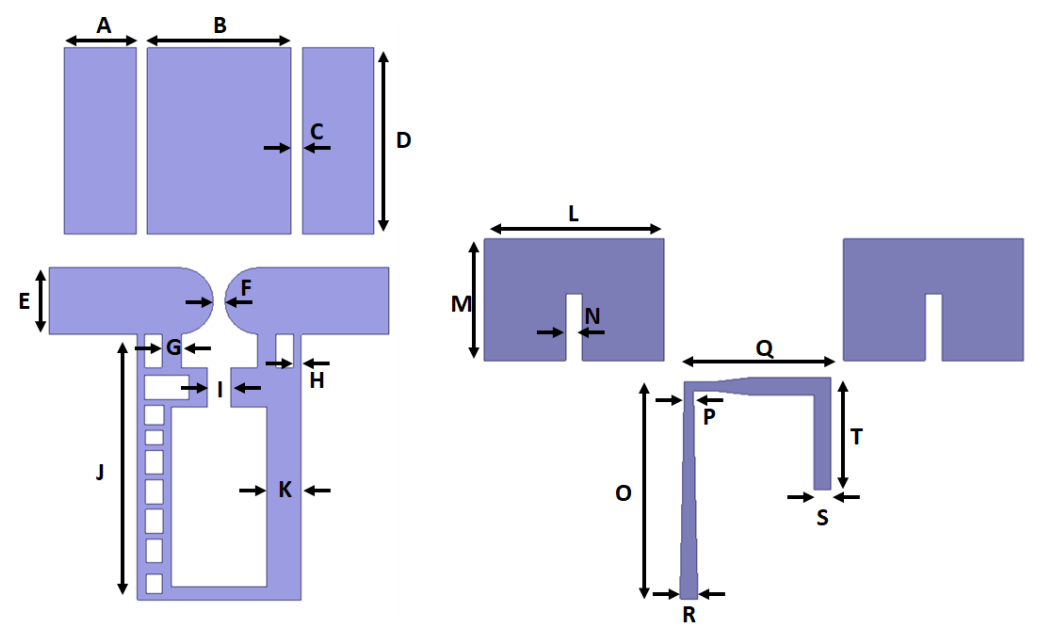

Figure 4.6: Fabrication details for egg-crate Ka-band TCDA.

Table 4.1: Dimensions (Units : mm) of V-band TCDA in Fig. 4.2

\begin{tabular}{|c|c|c|c|c|c|c|c|}
\hline $\mathbf{A}$ & 0.96 & $\mathbf{F}$ & 0.15 & $\mathbf{K}$ & 0.45 & $\mathbf{P}$ & 0.12 \\
\hline $\mathbf{B}$ & 1.92 & $\mathbf{G}$ & 0.25 & $\mathbf{L}$ & 2.4 & $\mathbf{Q}$ & 1.96 \\
\hline $\mathbf{C}$ & 0.15 & $\mathbf{H}$ & 0.1 & $\mathbf{M}$ & 1.62 & $\mathbf{R}$ & 0.23 \\
\hline $\mathbf{D}$ & 2.5 & $\mathbf{I}$ & 0.31 & $\mathbf{N}$ & 0.22 & $\mathbf{S}$ & 0.23 \\
\hline $\mathbf{E}$ & 0.89 & $\mathbf{J}$ & 3.55 & $\mathbf{O}$ & 37 & $\mathbf{T}$ & 1.5 \\
\hline
\end{tabular}



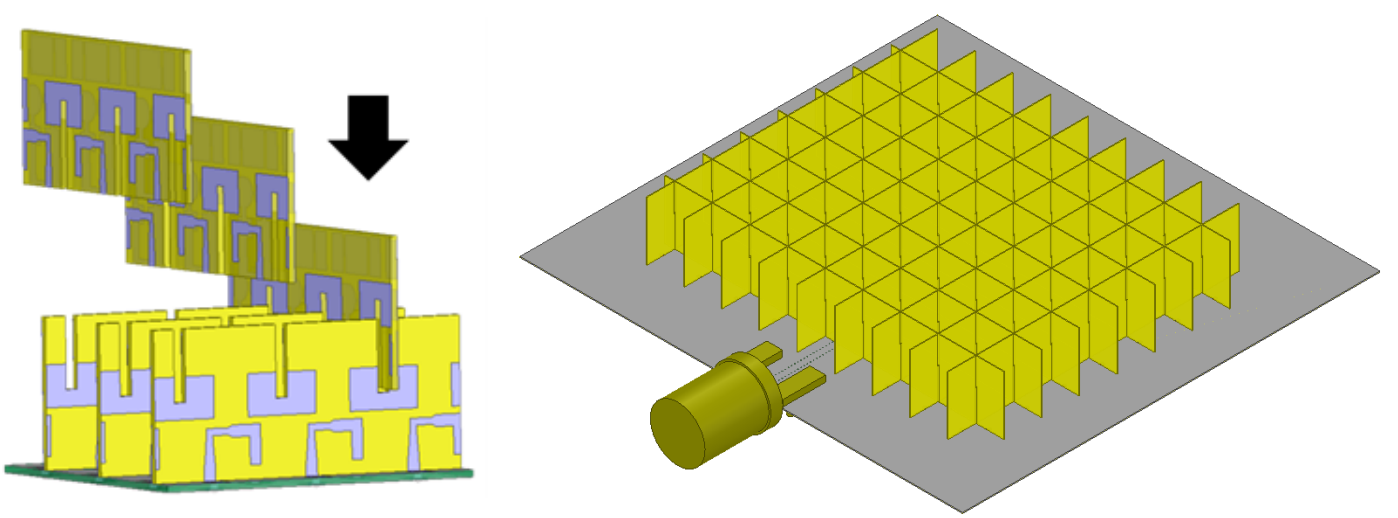

Figure 4.7: Pictorial representation of egg-crate Ka-band TCDA installment and array visualization

\subsubsection{Simplified Marchand Balun for $70 \mathrm{GHz}$ Operation}

The above design was scaled to $70 \mathrm{GHz}$ as depicted in Fig. 4.8. Notably, no perforations were included in the design of the V-band balun to create a simplified microstrip TCDA. This design improves on the 3:1 single polarized millimeter-wave TCDA presented in [NMV18]. Specifically, it enables 1) greater bandwidth, 2) simple construction with integrated balun, 3) dual polarization, 4) scanning to 60 ${ }^{\circ}$, and 5) ease of measurement for active feeding.

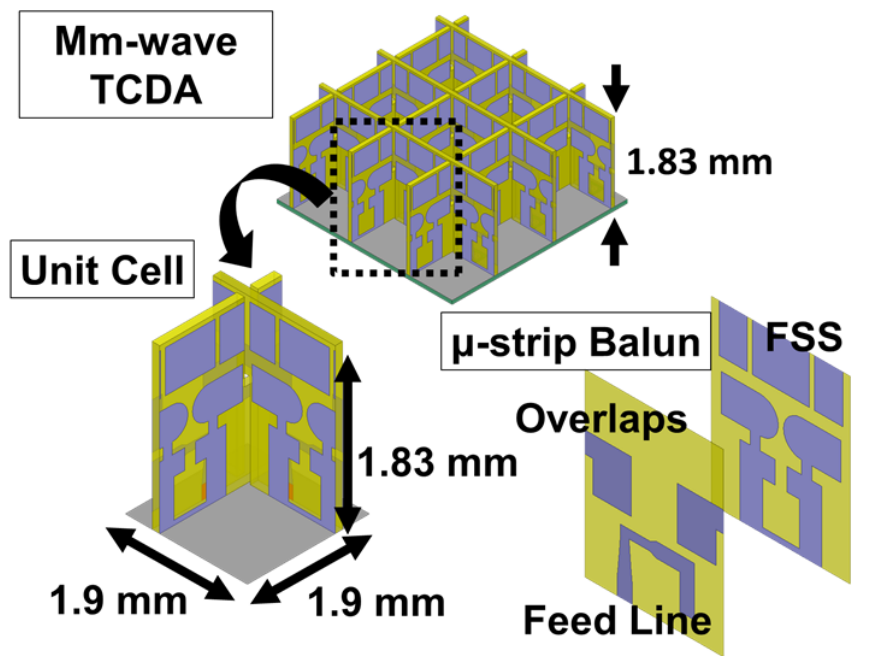

Figure 4.8: Simulated active VSWR of V-band TCDA infinite array unit cell 
For simplicity, an infinite array simulation was used to model the $8 \times 8$ finite element array using ANSYS HFSS v.19. The infinite array VSWR is given in Fig. 4.9. As seen, the array provides a VSWR $<3$ from $21 \mathrm{GHz}$ to $70 \mathrm{GHz}$, implying a broadside impedance bandwidth of 4:1. Notably, this is the first array to achieve scanning down to $60^{\circ}$ in all directions across $21 \mathrm{GHz}$ to $60 \mathrm{GHz}$. Full wave realized gain predictions are given in Fig. 4.10. These show that a near theoretical gain is achieved over the $5 \mathrm{G}$ bands, with more than $30 \mathrm{~dB}$ of cross-polarization purity down to $60^{\circ}$ scan angles.

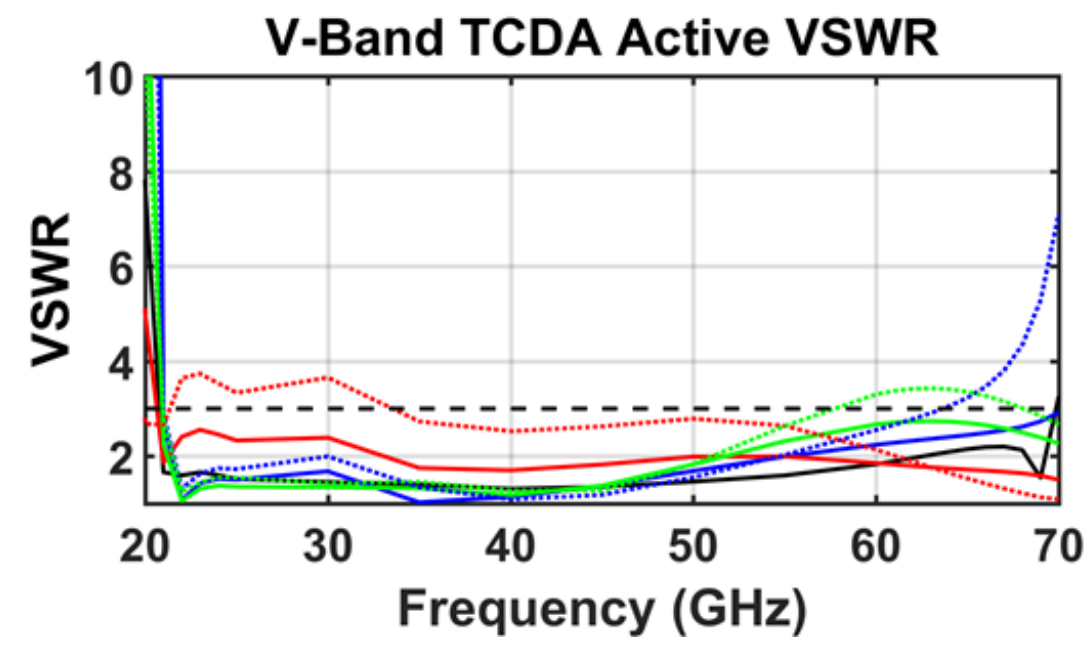

Figure 4.9: Simulated active VSWR of V-band TCDA infinite array unit cell
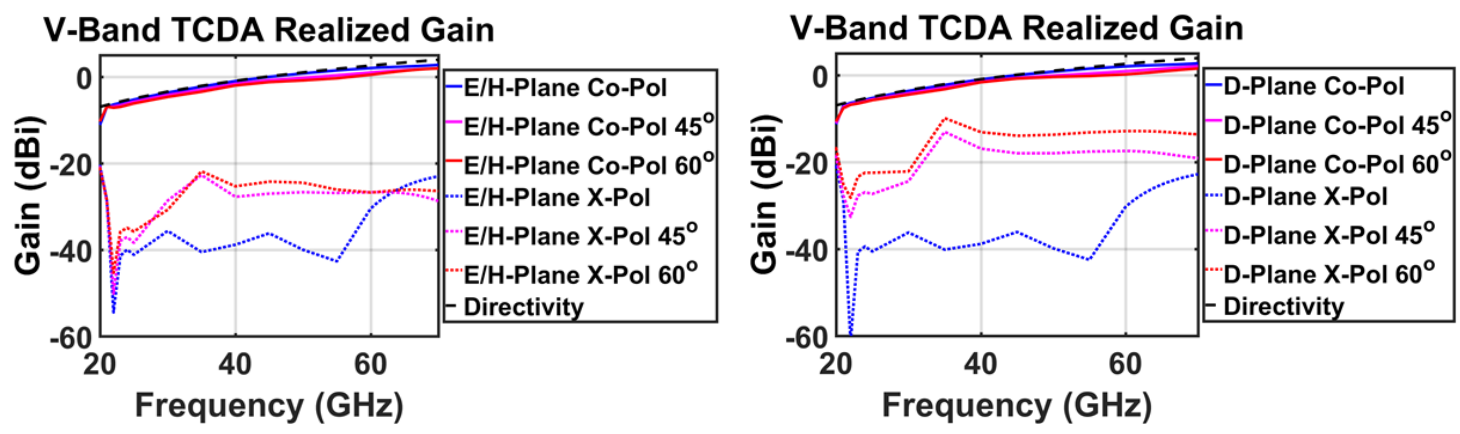

Figure 4.10: Realized gain in the principle planes (E/H) (left) and D-plane (right) for the simulated unit cell with scanning down to $60^{\circ}$ from broadside. 

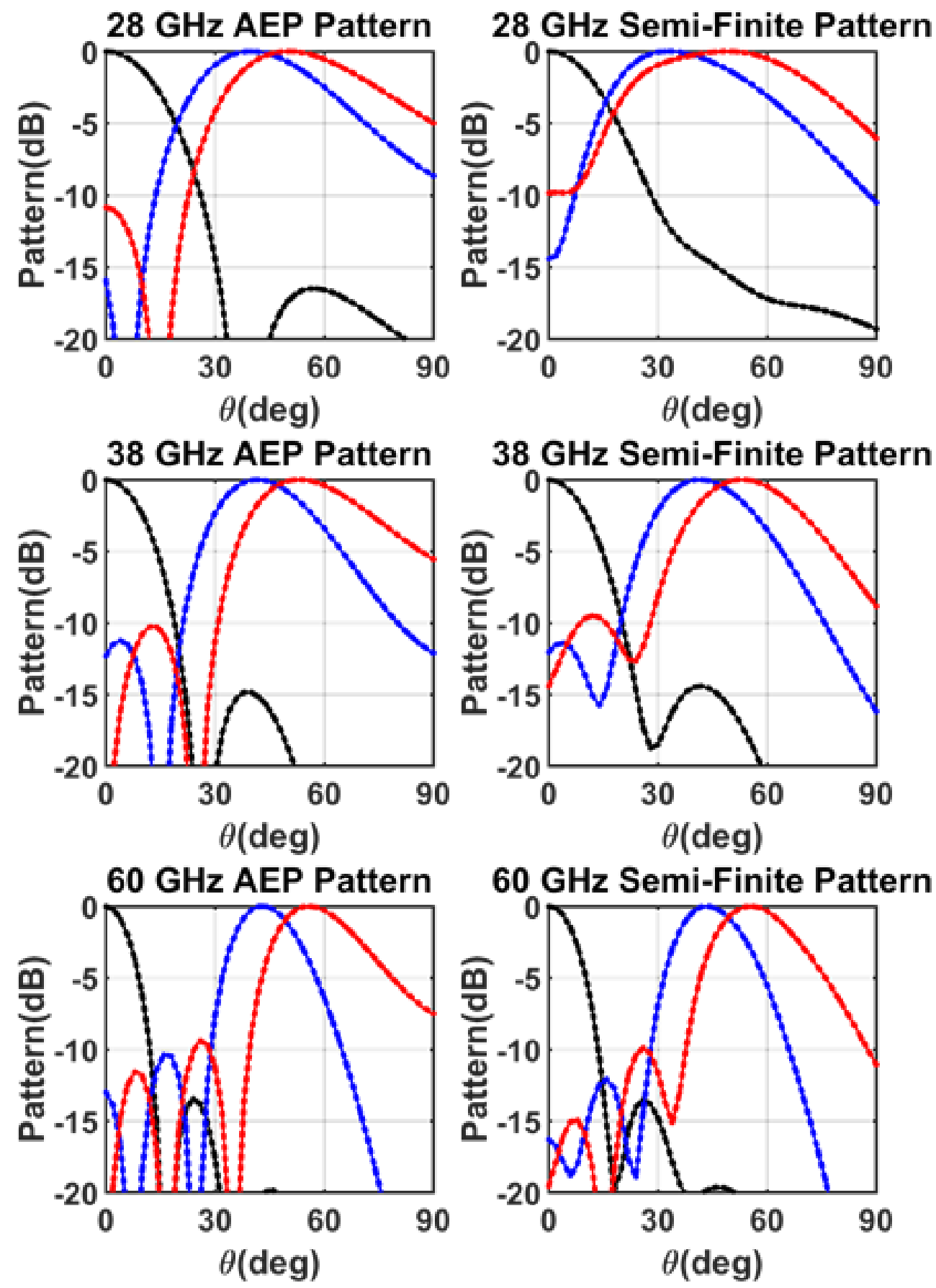

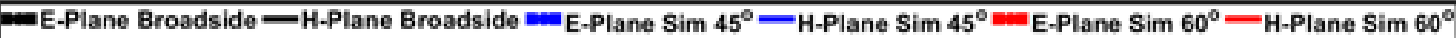

Figure 4.11: Realized gain patterns of V-band TCDA using AEP (left) and finite simulations (right) when scanning to $\theta=0^{\circ}, 45^{\circ}$, and $60^{\circ}$. 


\section{V-Band Egg-Crate Mm-Wave TCDA Fabrication}

The low-cost array employs standard PCB processes and dimensions to avoid the pitfalls of low yield processes like LTCC [LLJ+19]. The antenna in Fig. 4.12(left) consists of one Rogers $4003\left(\epsilon_{r}=3.6\right)$ laminate with features as shown in Fig. 4.12(right). The minimum metal tolerance is $0.0762 \mathrm{~mm}$ (3 mil) in accordance with commercial PCB processes, as detailed in Fig. 4.13 and Table 4.2. As depicted, the 76.2 micron metal traces were accurately printed using the LPKF Protolaser U4 laser etcher at FIU's RFCOM Lab. For reference the stand-alone $8 \times 8$ array in Fig. 4.14 is placed next to a US quarter. This integrated array is an attractive candidate for mobile $5 \mathrm{G}$ platforms due to its small size and weight $(<2 \mathrm{~mm}$ tall and $<1 \mathrm{~g}$ ), with almost $50 \mathrm{GHz}$ of available bandwidth. The UWB feed structure used to excite the array is discussed in the following sections.

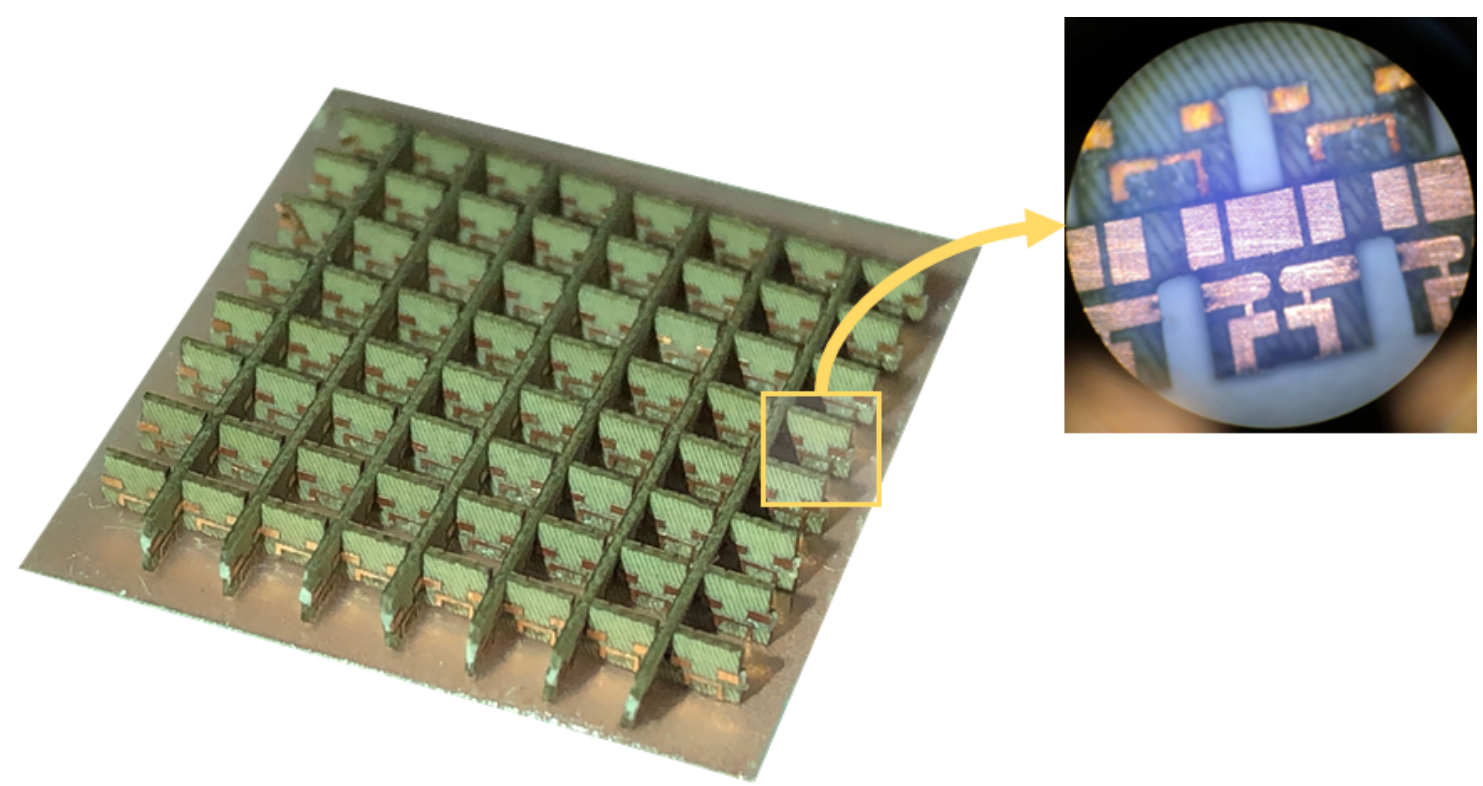

Figure 4.12: Fabricated egg-crate mm-wave TCDA 


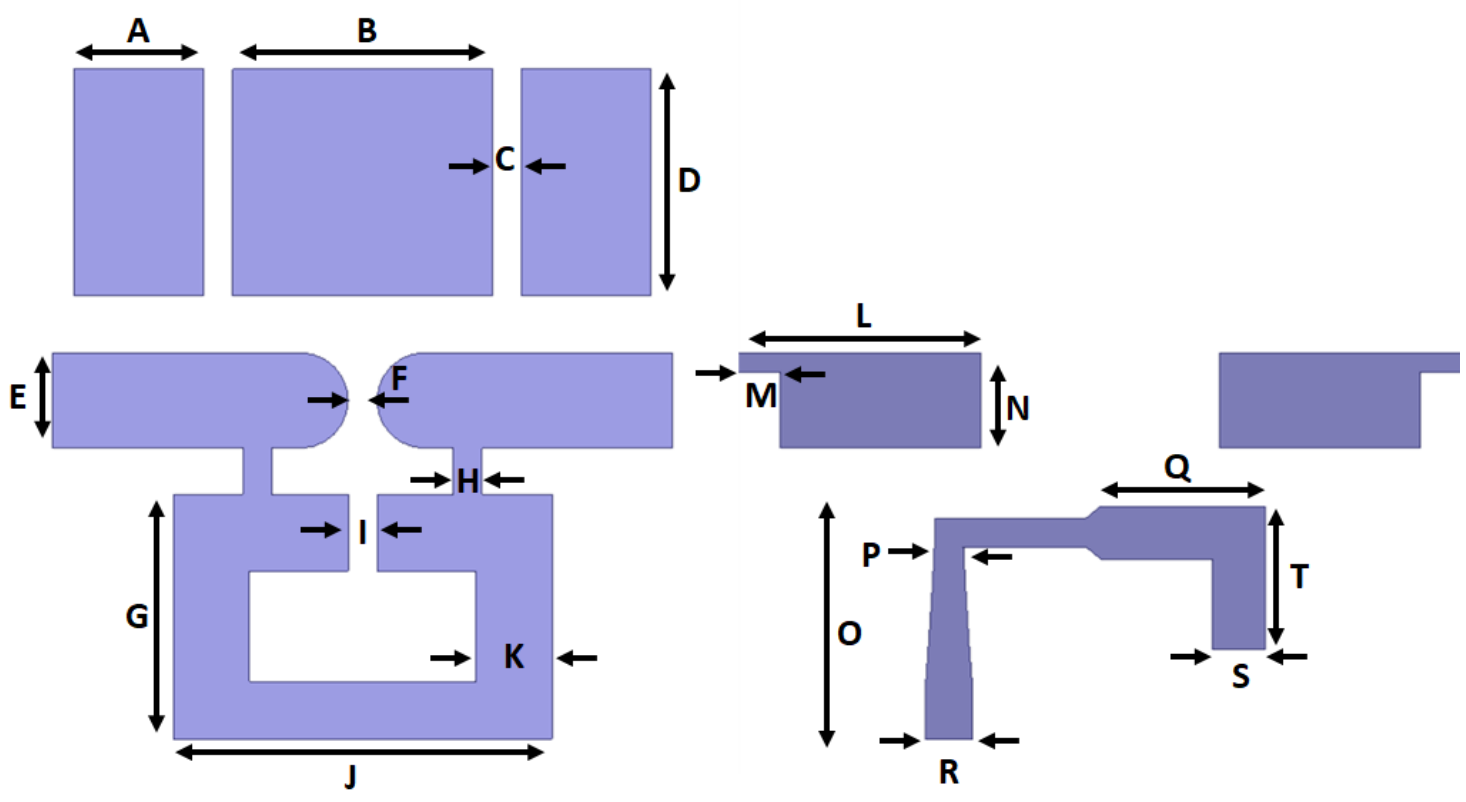

Figure 4.13: Fabrication details for egg-crate mm-wave TCDA.

Table 4.2: Dimensions (Units : $\mathrm{mm}$ ) of V-band TCDA in Fig. 4.8

\begin{tabular}{|c|c|c|c|c|c|c|c|}
\hline $\mathbf{A}$ & 0.34 & $\mathbf{F}$ & 0.07 & $\mathbf{K}$ & 0.2 & $\mathbf{P}$ & 0.07 \\
\hline $\mathbf{B}$ & 0.69 & $\mathbf{G}$ & 0.65 & $\mathbf{L}$ & 0.63 & $\mathbf{Q}$ & 0.43 \\
\hline $\mathbf{C}$ & 0.07 & $\mathbf{H}$ & 0.07 & $\mathbf{M}$ & 0.1 & $\mathbf{R}$ & 0.12 \\
\hline $\mathbf{D}$ & 0.6 & $\mathbf{I}$ & 0.07 & $\mathbf{N}$ & 0.25 & $\mathbf{S}$ & 0.14 \\
\hline $\mathbf{E}$ & 0.25 & $\mathbf{J}$ & 1.0 & $\mathbf{O}$ & 0.62 & $\mathbf{T}$ & 0.38 \\
\hline
\end{tabular}

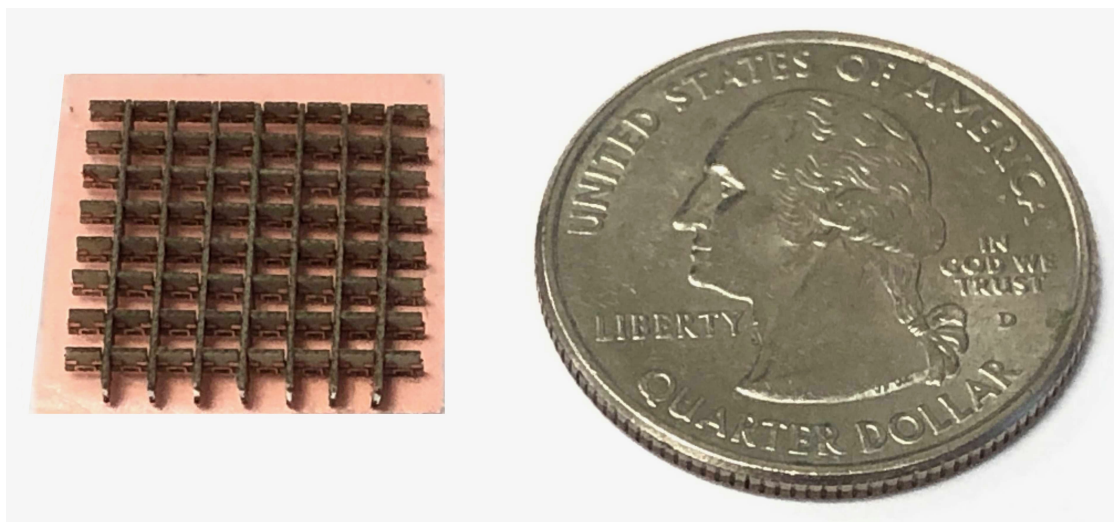

Figure 4.14: Fabricated egg-crate mm-wave TCDA with size referenced to a US quarter 


\subsection{Differential 5G Millimeter-Wave Array}

As previously mentioned, recent advancements in differential RF front-end components, such as commercial push-pull amplifiers provide significant increases in the operational bandwidths of balanced 5G transceivers [Spi16]. These signals have the following attributes [Poz12]:

- lower noise in the transceiver chain

- high dynamic range and linearity

- lower distortion in response to noise and interference from power supplies

- suppressed even-order harmonic distortions

However, as discussed in Chapter 3, differentially fed antenna are plagued by common-mode resonances when scanning in the E-plane down to low angles from boresight. In [BSC93, EO60, LLK04, dLAGGP ${ }^{+}$10], UWB arrays with differential feeds were presented. However, these arrays exhibited common-mode resonances when scanning in the E-plane nearing angles down to $45^{\circ}$. Again, these commonmodes arise for particular scan angles and frequencies in the differential feed lines due to destructive mutual coupling between elements in presence of a third conductor (i.e. ground plane). The effect of these modes is exacerbated when they radiate, causing a significant reduction in the efficiency and impedance bandwidth of the balanced antenna structure [S. 08]. Hence, the major challenge in the design of full differential radios is the reduction of these common-mode currents present at the aperture when scanning.

The most prevalent way to suppress scan-dependent common-modes is to employ a balanced-to-unbalanced feed (i.e. balun), thereby avoiding differential feeding altogether [DSV13c]. However, this requires a single ended RF front-end which is 
not compatible with differential power amplifiers and would require additional hardware. Another method employs conductive E-Plane walls to disrupt the fields between neighboring linear arrays [BSC93, EO60], but this approach is limited to single polarized arrays only. Alternatively, resistive terminations can be exploited to attenuate the common-modes, at the cost of significant losses [LLK04, dLAGGP $\left.{ }^{+} 10\right]$. Most importantly, there are no UWB phased arrays with differential feeds at mmwaves in the literature.

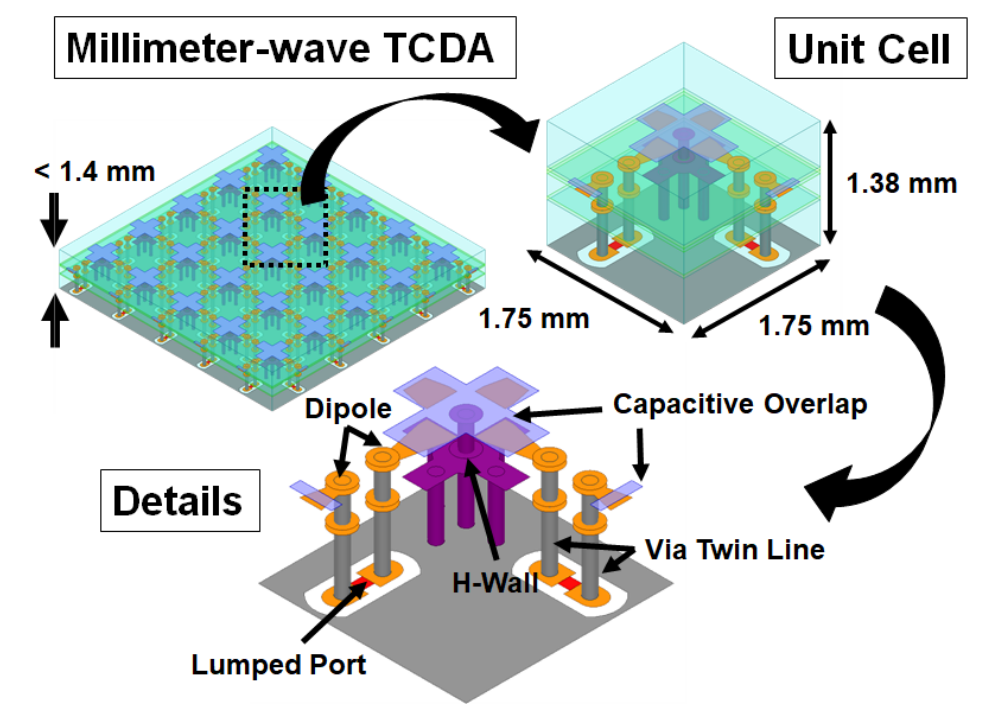

Figure 4.15: Pictorial representation of a Dual polarized Differential TCDA array with Unit Cell of the design.

In this section, we present a dual-polarized UWB differentially fed array for mmwaves, shown in Fig. 4.15. The array is developed for operation across $\mathrm{K}$ to $\mathrm{W}$ and 5G bands (viz. 22-80 GHz). To remove the E-Plane scan resonance, a novel multilayer dual-polarized H-wall structure, shown in Fig. 4.15, is introduced to eliminate the scan-dependent common-modes. In this section, an optimized dual-polarized phased array with 1) differential feeding at mm-waves, 2) low angle scanning capability, and 3) simple low cost fabrication with standard PCB processes is presented. 


\subsubsection{Differentially Fed TCDA Design}

A just mentioned, the printed Marchand balun feeding structure for the TCDA is not suitable for mm-wave transmit applications. Instead, we expand on the differential S and Ku band TCDAs presented in Chapter 3, for adaptation to mm-waves. Improving on the 3:1 single polarized mm-wave balun-fed TCDA [NMV18], this differential design uses a dual-polarized topology for full polarization diversity. The simple design uses symmetric coplanar dipoles with a cross-shaped overlapping elements to introduce capacitive coupling in a planar low profile of $1.38 \mathrm{~mm}$, as depicted in Fig. 4.15. The tightly coupled dipoles are excited by the differential twin 4 mil diameter via feed in Fig. 4.16 (right), where symmetry between the feed lines is maintained for balanced transmission of opposite phase signals across a wide bandwidth. A dielectric superstrate improves impedance matching when scanning to $60^{\circ}$. As seen in Fig. 4.17(a), a broadside VSWR $<2$ is achieved across the band. However as expected, a resonance does occur when scanning in the E-plane. To remove that resonance, the multi-layer dual-polarized $\mathrm{H}$-wall structure detailed in Fig. 4.15 was designed to disrupt the scan-dependent common-modes and remove them from the band. This is similar to the concept in [BSC93], but implemented in a dual-polarization.

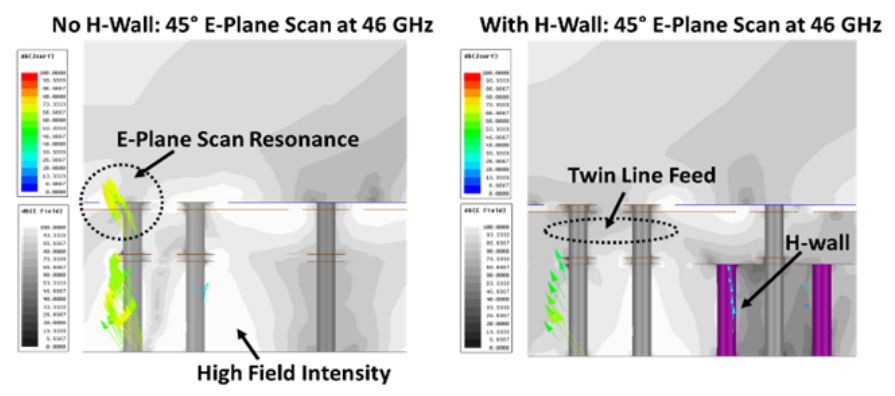

Figure 4.16: Magnitude of the Electric Field $E$ and Electric Current $J$ vectors in the E-Plane for $45^{\circ}$ scanning. It shows that the differential feed lines radiate (left) and the removal of common-mode resonance with the H-Wall Structure (right). 


\subsubsection{Removal of E-Plane Scan Resonance}

As depicted in Fig. 4.16 (left), the feed lines cause a resonance when scanning in the E-plane. These feed lines radiate due to the common-modes introduced by the high intensity fields between neighboring unit cells in the E-Plane. Fig. 4.16 (right) shows that the fields in the substrate of the dipoles are confined between the feed lines with lower intensity for efficient radiation even when scanning to $45^{\circ}$. Full wave simulations of the optimized array in an infinite array setup show that the D-TCDA with H-wall exhibits VSWR $<3$ across 22-80 GHz and scanning down to $60^{\circ}$, as depicted in Fig. 4.17(b). Broadside gain predictions are given in Fig. 4.18, showing that a near theoretical realized gain is achieved with polarization purity greater than $40 \mathrm{~dB}$.
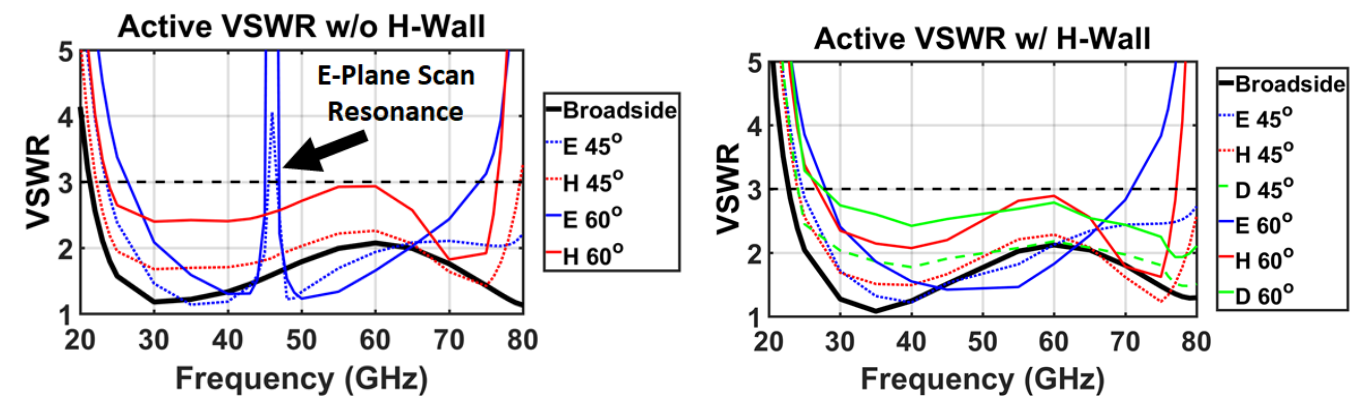

Figure 4.17: VSWR of D-TCDA using infinite array simulation (left) without HWall and (right) with H-Wall.
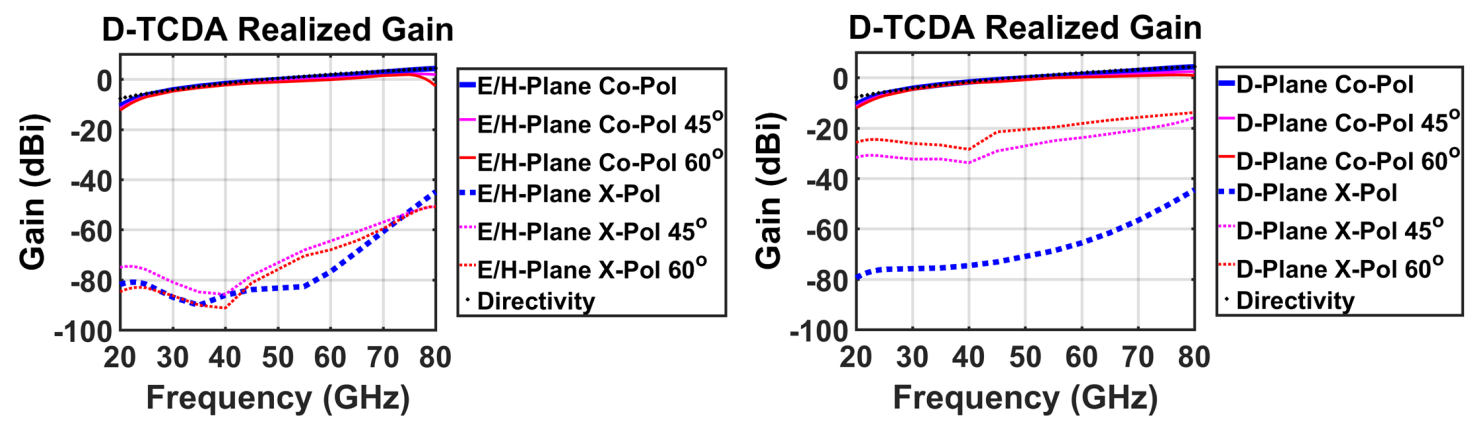

Figure 4.18: Realized gain in the principle planes $(\mathrm{E} / \mathrm{H})$ (left) and D-plane (right) for the simulated unit cell with scanning down to $60^{\circ}$ from broadside. 
The simulated patterns in Fig. 4.19 (left) validate beam steering down to $60^{\circ}$ from broadside in the principle E/H planes using the Active Element Pattern (AEP) method [Poz94]. Semi-finite simulations show similar results in Fig. 4.19. This method models a finite 10 element linear array in one plane, with a periodic boundary condition in the other. Doing so, finite and edge effects can be observed in one plane without computationally taxing simulations. The symmetry of the design produces an identical pattern in the $\mathrm{E}$ and $\mathrm{H}$ planes.
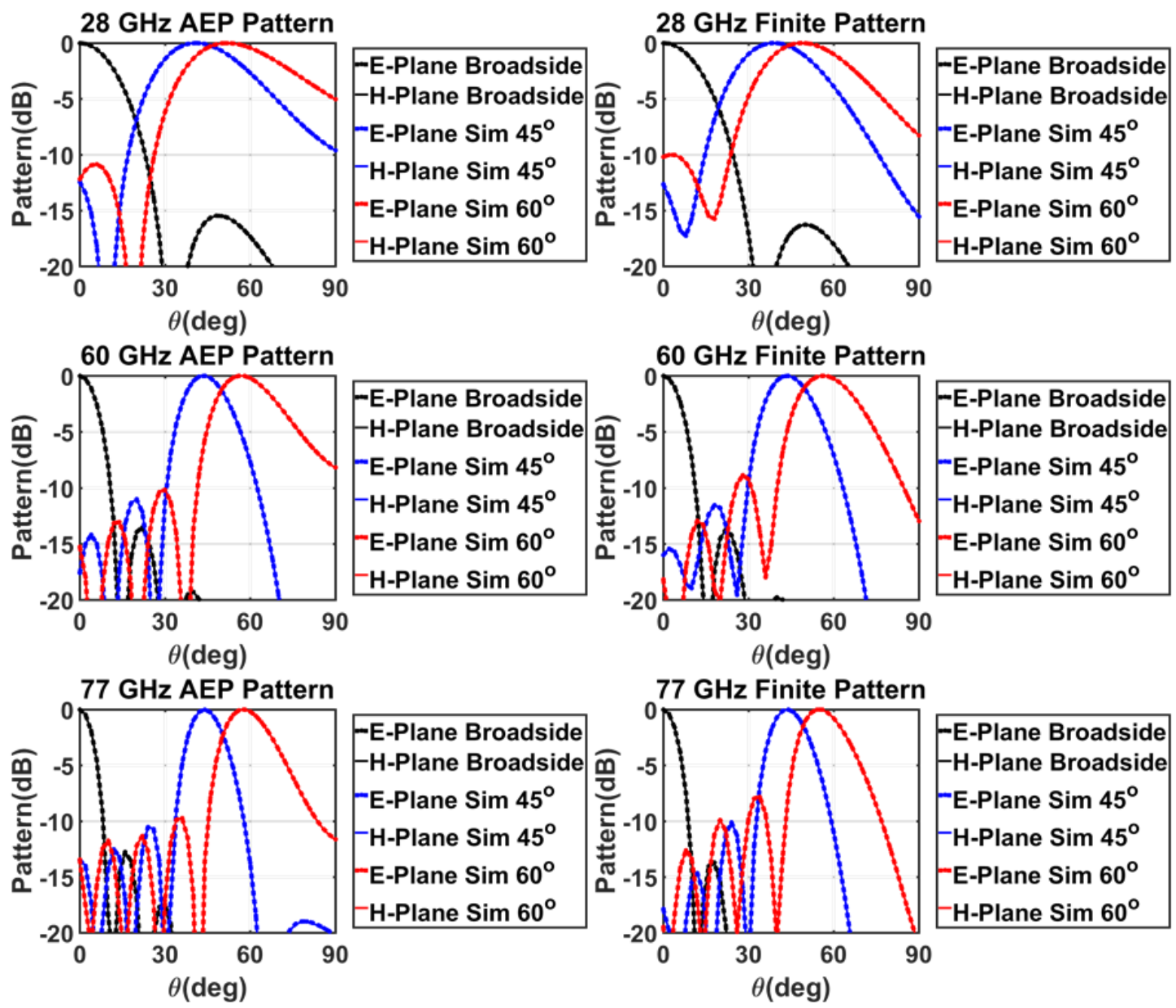

Figure 4.19: Realized gain patterns of D-TCDA using AEP (left) and finite simulations (right) with scanning to $\theta=0^{\circ}, 45^{\circ}$, and $60^{\circ}$. 


\subsubsection{Fabrication of Differential mm-Wave TCDA}

The differential low-cost array also employs standard PCB processes and dimensions.

The antenna in Fig. 4.20 was constructed from three layers of Rogers $5880\left(\epsilon_{r}=2.2\right)$ laminates with layers as shown in Fig. 4.21. The drilled 4 mil vias are used to feed the dipole elements. Notably, the minimum metal tolerance is $0.0762 \mathrm{~mm}$ (3 mil) in accordance with commercial PCB processes. These $76.2 \mu \mathrm{m}$ metal traces were accurately printed using the LPKF Protolaser U4 laser etcher at FIU's RFCOM Lab. For reference the stand-alone $10 \times 10$ array in Fig. 4.20 is shown next to a US quarter. Again, this array is an attractive candidate for mobile $5 \mathrm{G}$ platforms due to its small size and weight $(<1.5 \mathrm{~mm}$ tall and $<1 \mathrm{~g})$.

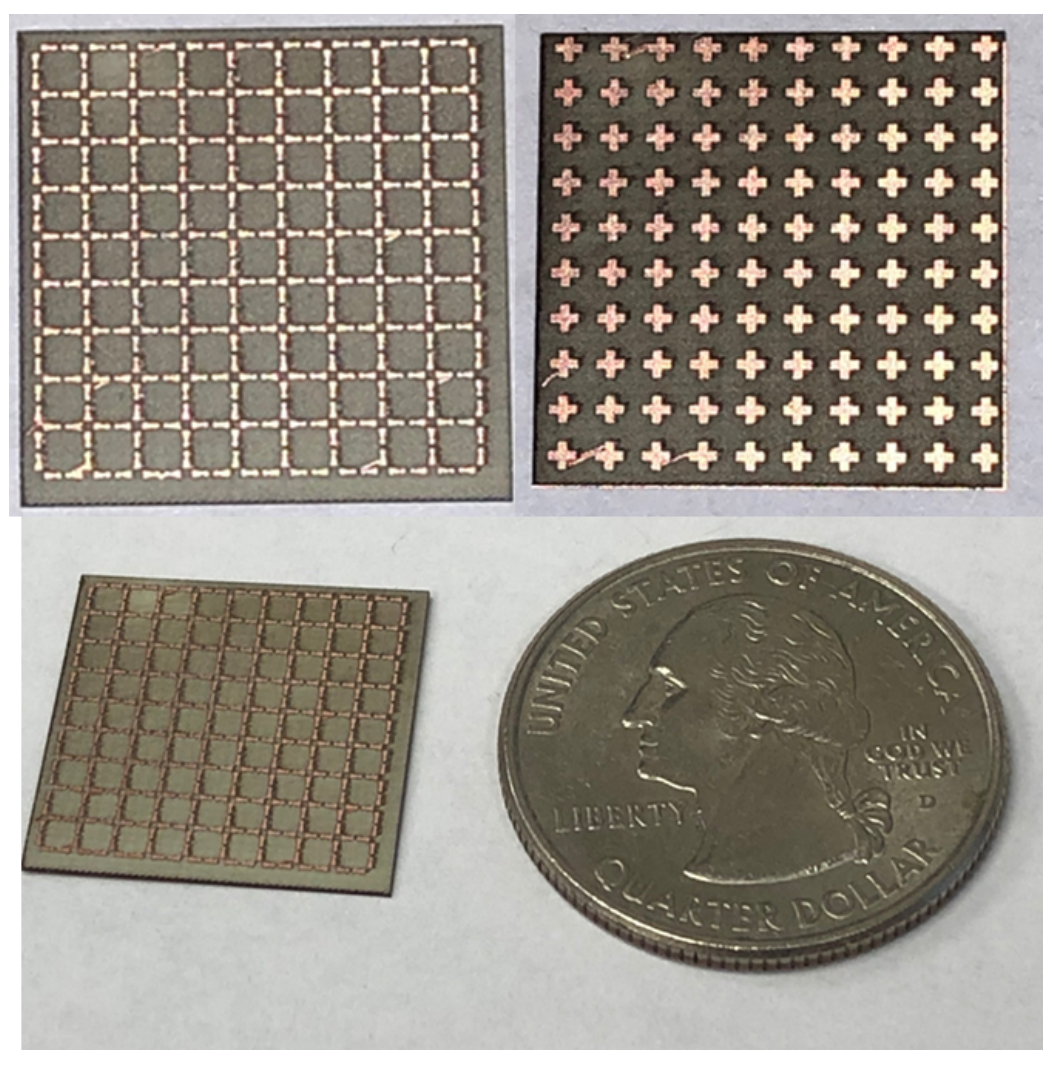

Figure 4.20: Example $10 \times 10$ fabricated prototype next to a U.S. Quarter. 


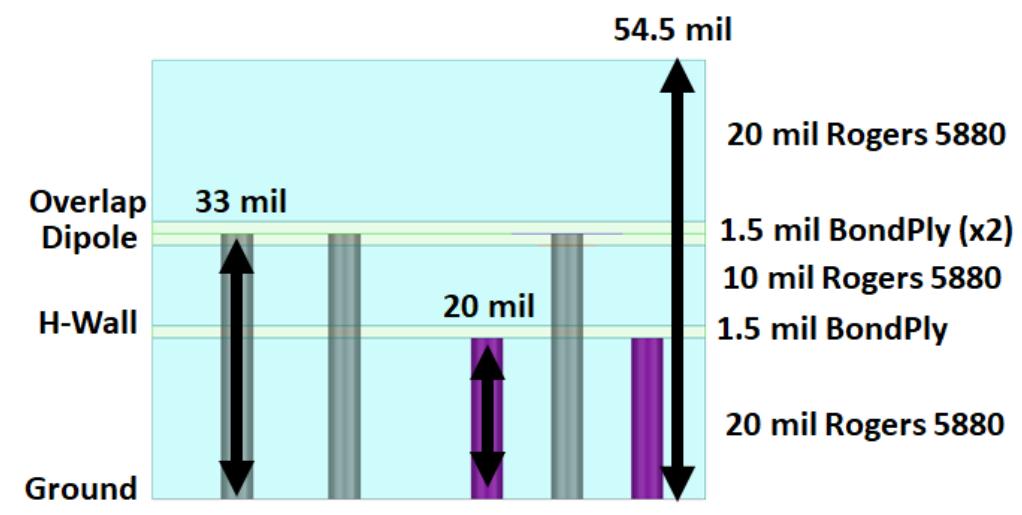

Figure 4.21: Stackup showing metal layers and via placement for PCB based differential TCDA.

\subsection{Feeding Approaches and Fabrication}

Measurement of the mm-wave arrays is not a trivial task due to active feeding challenges. The pressing issue with mm-wave phased arrays is the implementation of a feed board to accommodate a densely populated array using impedance-matched low-inductance leads. To measure the performance of our TCDAs across almost 60 $\mathrm{GHz}$ of bandwidth, three test structures were designed, each with the capability to excite the entire 22-80 GHz bandwidth with $S_{11}<-10 \mathrm{~dB}$ and loss of $<1 \mathrm{~dB}$ per stage. This test structure will measure a single element and an 8 element linear array using printed resistive sheets to match-terminate the remaining elements. Each feed network is comprised of:

- Ball-Grid-Array vertical integration

- UWB balun for compatibility with single-ended measurement equipment (differential antenna only)

- $28 \mathrm{GHz}$ fixed beam power combiner to show the finite effects of an 8 element array 


\subsubsection{Ball-Grid-Array}

A Ball-Grid-Array (BGA) is employed as a low cost solution for vertical integration of an active phased array onto the feed board. Ball-grid-arrays are comprised of a mixture of tin and lead that melts at lower temperatures than the printed conductors to achieve a stable RF connection. Notably, these BGA interconnects have minimal effect on impedance matching. This is demonstrated by the data in Figs. ??.
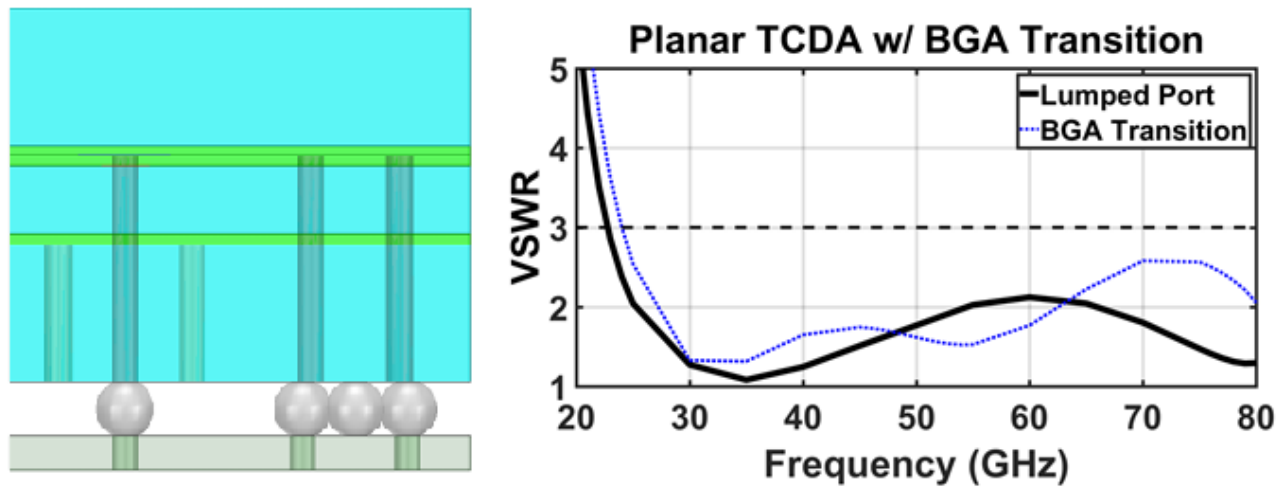

Figure 4.22: Effect of BGA interconnect on differential TCDA.

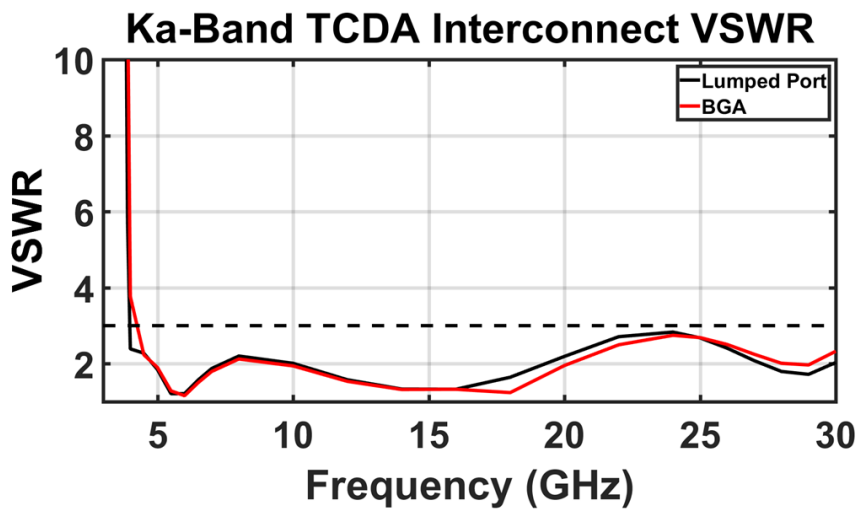

Figure 4.23: Effect of BGA interconnect on Ka-band TCDA. 


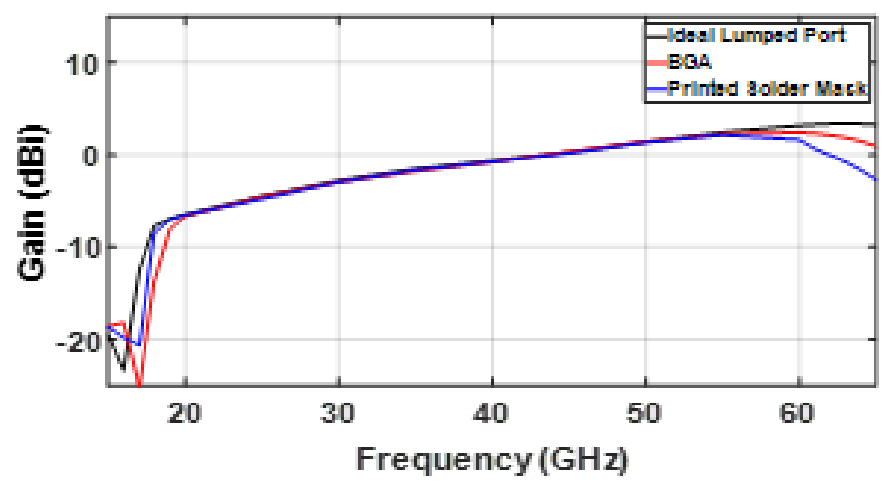

Figure 4.24: Effect of BGA interconnect on V-band TCDA.

\subsubsection{UWB Balun}

To measure the differential array with the available single-ended measurement equipment the UWB balun in Fig. 4.25 was designed and integrated into the feed structures. As opposed to the wideband Marchand balun and tapered balun used to excite the TCDA previously presented in this dissertation, an alternate design was implement using microstrip technology. The balun was designed with the feed board on a 5 mil Rogers 3003 substrate. This balun transfers the balanced signal into a single-ended line with a tapered ground plane shorted to one differential arm by a 4 mil diameter via. As depicted in Fig. 4.25, the balun gives $S_{11}<-10 \mathrm{~dB}$ over the 22-80 GHz band of the differential array discussed above.
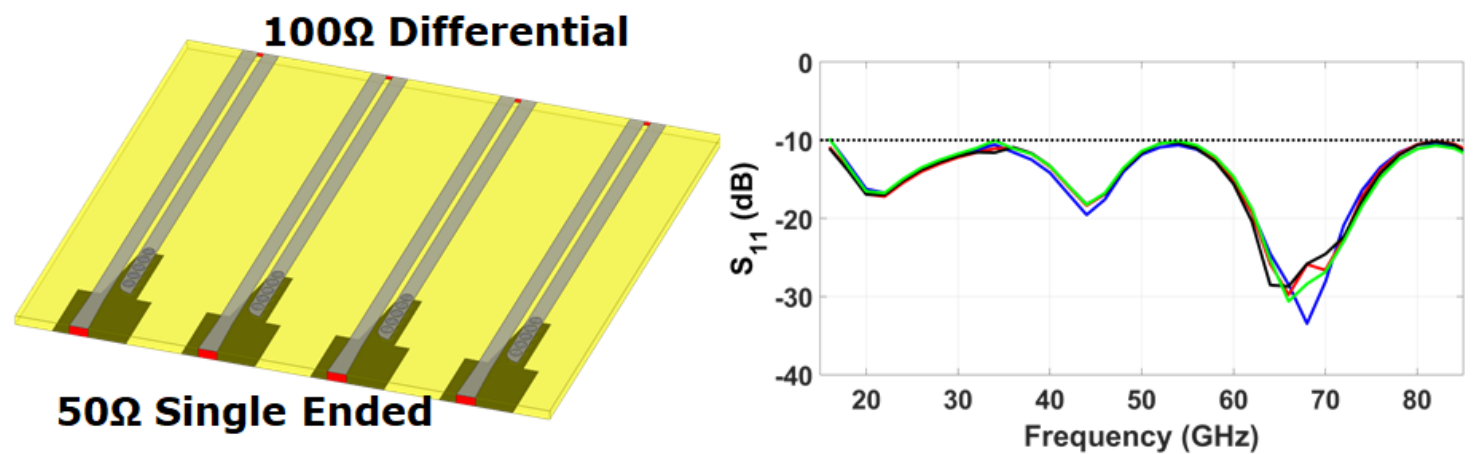

Figure 4.25: Planar UWB Balun (left) with simulated performance (right). 


\subsubsection{Wilkinson Divider}

To see finite effects of the V-band arrays, a 2.4mm coax launcher (for use $<50$ $\mathrm{GHz}$ ) was used to excite a a $28 \mathrm{GHz}$ Wilkinson 8-way power combiner. An ideal Wilkinson power divider has the S-Parameters as given in Eq. 4.1, with equal power splitting and matched loads. Given that the Wilkinson is passive and reciprocal, it can easily be adapted to a power combiner. A combiner was therefore designed for both the V-band balun-fed and the differential TCDAs using a 5 mil Rogers 3003

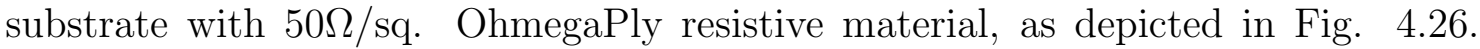
The Wilkinson combiner for the differential array implemented an array of baluns to transfer the balanced signal to a single-ended microstrip line before being power combined. The simulated return loss of these power combiners were more than 10 $\mathrm{dB}$ at $28 \mathrm{GHz}$.

$$
[S]=\frac{-j}{\sqrt{2}}\left[\begin{array}{lll}
0 & 1 & 1 \\
1 & 0 & 0 \\
1 & 0 & 0
\end{array}\right]
$$
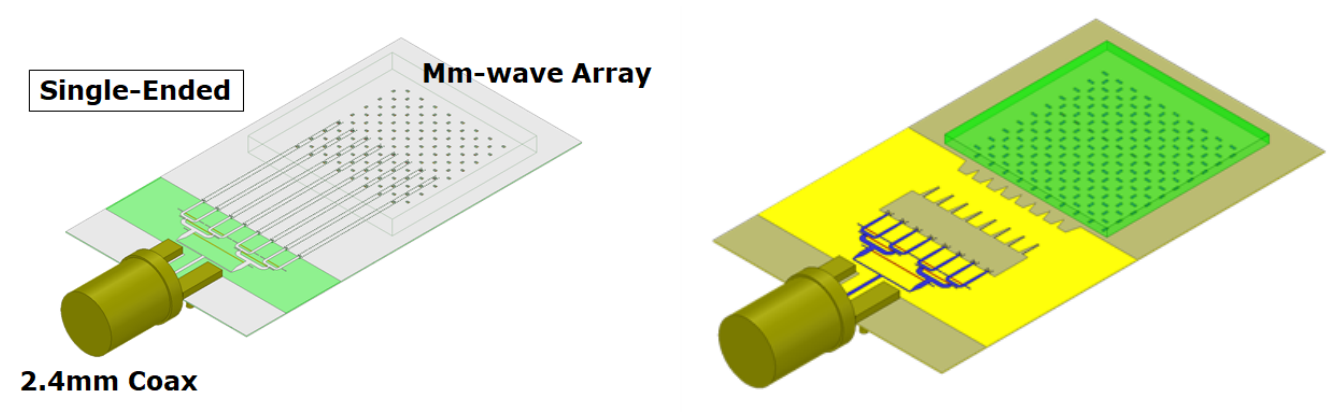

Figure 4.26: $28 \mathrm{GHz}$ power combiner for Balun-fed array (left). $28 \mathrm{GHz}$ power combiner for with integrated baluns for differential array (right). 


\subsubsection{Ka-Band Array Feed}

To measured the Ka-Band array, a feed board was designed using a $2.4 \mathrm{~mm}$ coaxial launcher, with the top side of the board acting as the array ground plane. The signal path of this feed network is given in Fig 4.27. After the BGA connection is made between the antenna cards and a feed pad on the top layer of the feed structure, a via directs the signal down to the back side of the feed structure. Then, the microstrip signal on the back side is directed under the ground plane to a via that leads past the ground plane and to the $2.4 \mathrm{~mm}$ launcher. The simulated results of this can be found in Fig. 4.28.

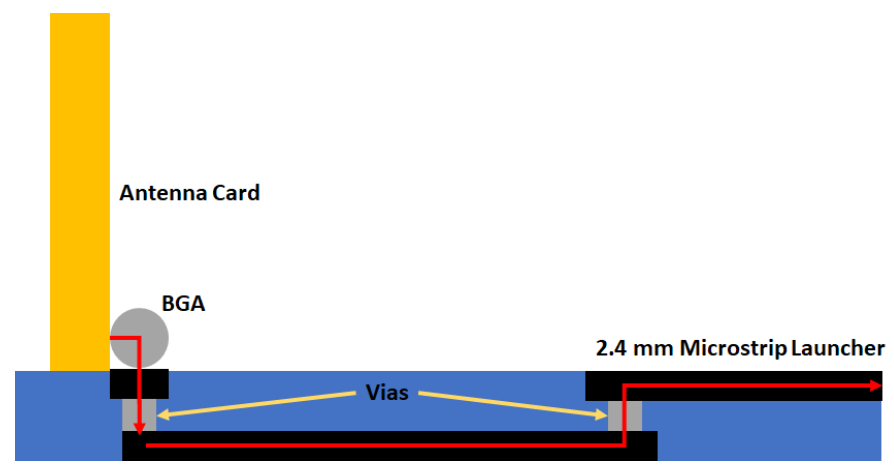

Figure 4.27: Signal Path of Ka-Band feed with 2.4mm connector.
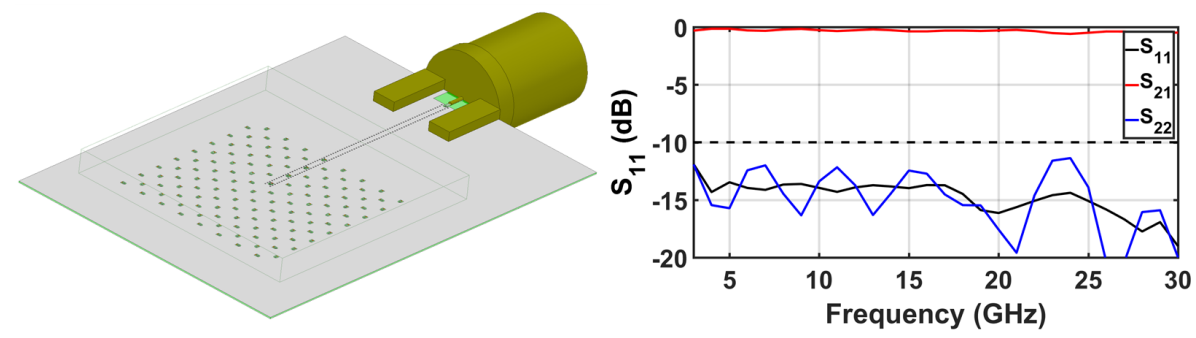

Figure 4.28: Ka-Band feed with 2.4mm connector. 


\subsubsection{V-Band Array Feeds}

There is a challenge associated with available measurement equipment for UWB 5G arrays. As a result, many works in the literature rely on waveguide fed probes, using a ground-signal-ground (GSG) transmission line feed point for measurement. However, this technique has the downside waveguide bandwidth limitations. For instance, this approach would require at least 4 separate waveguides to cover the 22-80 GHz spectrum. Further, there is a requirement for several coaxial connectors to cover these bands continuously. Table 4.3 outlines the available options for measurement in the $18-110 \mathrm{GHz}$ bands.

Table 4.3: Measurement options for the 22-80 GHz spectrum.

\begin{tabular}{|c|c|c|c|c|c|}
\hline Type & $\begin{array}{c}\text { Frequency } \\
\text { Range }(\mathbf{G H z})\end{array}$ & Mates & Type & $\begin{array}{c}\text { Frequency } \\
\text { Range } \mathbf{( G H z )}\end{array}$ & Mates \\
\hline WR42 & $18-26.5$ & - & $1.0 \mathrm{~mm}$ & $\mathrm{dc}-110$ & $1.0 \mathrm{~mm}$ \\
\hline WR34 & $26.5-40$ & - & $1.85 \mathrm{~mm}$ & $\mathrm{dc}-70$ & $2.4 \mathrm{~mm}$ \\
\hline WR28 & $33-50$ & - & $2.4 \mathrm{~mm}$ & $\mathrm{dc}-50$ & $1.85 \mathrm{~mm}$ \\
\hline WR19 & $40-60$ & - & $2.92 \mathrm{~mm}$ & $\mathrm{dc}-40$ & $3.5 \mathrm{~mm} / \mathrm{SMA}$ \\
\hline WR15 & $50-75$ & - & $3.5 \mathrm{~mm}$ & $\mathrm{dc}-34$ & $2.92 \mathrm{~mm} / \mathrm{SMA}$ \\
\hline WR12 & $60-90$ & - & SMA & dc-24 & $2.92 \mathrm{~mm} / 3.5 \mathrm{~mm}$ \\
\hline WR10 & $75-110$ & - & - & - & - \\
\hline
\end{tabular}

Measurements at V-band must also address the common-place discontinuity in available equipment at $50 \mathrm{GHz}$. To overcome the lack of readily available equipment, this dissertation presents three measurement techniques for UWB arrays at mmwaves:

1. Splitting the bands using separate feeds for $<50 \mathrm{GHz}$ and $>50 \mathrm{GHz}$

2. Single feed with a diplexer to filter the high and low bands to separate $<50$ $\mathrm{GHz}$ and $>50 \mathrm{GHz}$ outputs

3. Single feed board with an UWB $1.0 \mathrm{~mm}$ connector to a single output The designs and performance of each case is discussed below. 


\section{Separate Split-Band Feeds}

To feed each V-band array, two separate feed boards can be used based on available equipment. This split-band approach uses two different commonly available measurement types: 1) $2.4 \mathrm{~mm}$ coax for $<50 \mathrm{GHz}$ 2) waveguide $150 \mu \mathrm{m}$ pitch groundsignal-ground (GSG) probe for $>50 \mathrm{GHz}$. This split-band approach provides the lowest cost solution to obtain important measurements for 5G and mm-wave systems. The signal path follows that of Fig. 4.28, but using different output launchers (2.4 $\mathrm{mm}$ and GSG Probe), as shown in Fig. 4.29. The simulations are also given in Fig. 4.29. As seen, $S_{11}<-10 \mathrm{~dB}$ with a maximum loss of $<2 \mathrm{~dB}$. This will be de-embedded to compute the actual measured array gain.

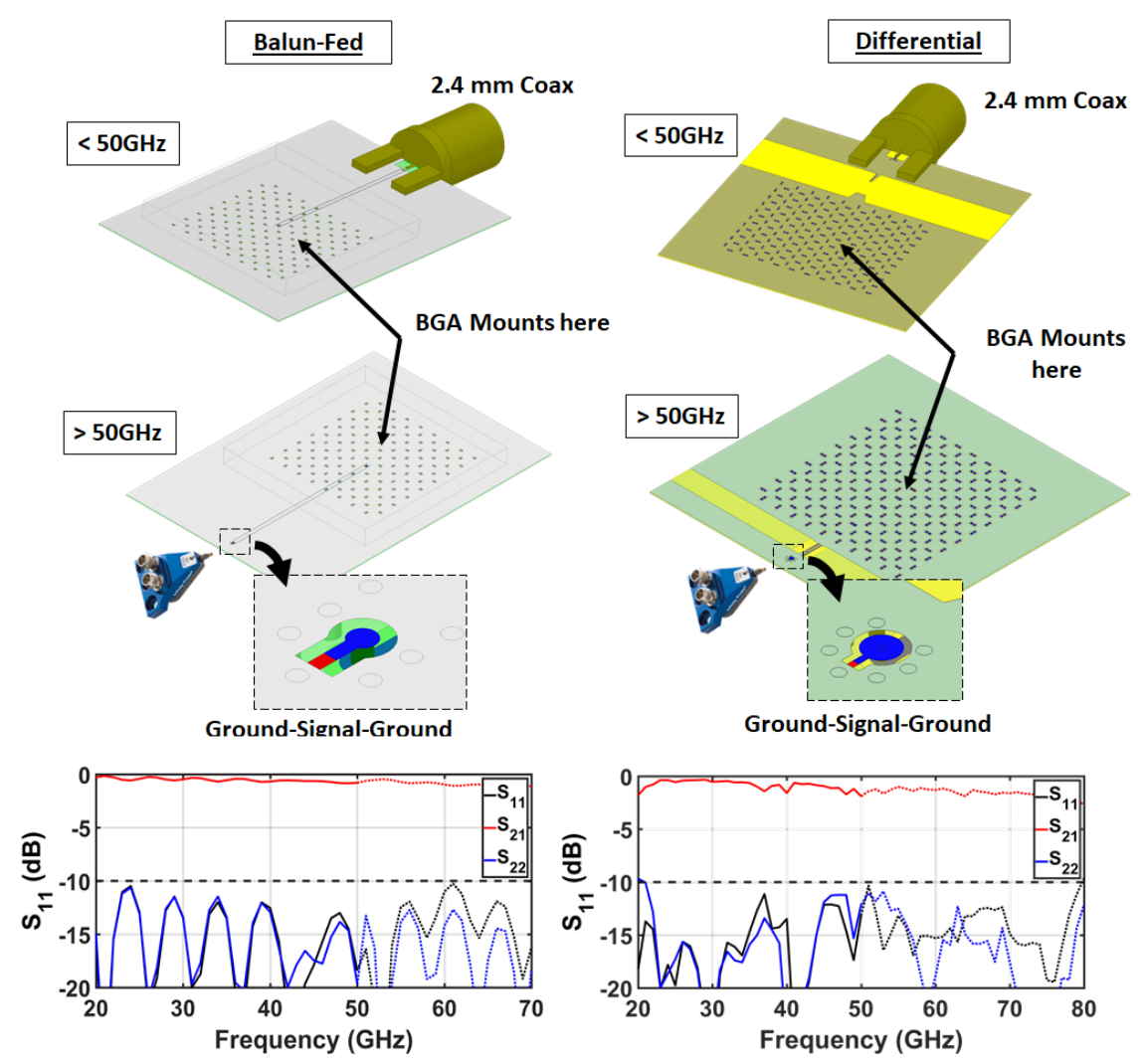

Figure 4.29: Simulated performance (bottom) of the balun-fed (left) and differential (right) V-band Split-band array feeds. The $2.4 \mathrm{~mm}$ feed for the low-band (top) and the ground-signal-ground feed for the high-band (middle) are also included. 


\section{Diplexer Split-Band Feed}

Another measurement approach is to characterize one fabricated prototype across the entire bandwidth with two outputs (viz. $2.4 \mathrm{~mm}$ coax and GSG probe). A Wilkinson divider can split the signal to the two outputs, but requires matched loads on the unused ports. Therefore, a diplexer must be employed. A diplexer is a threeport integrated low pass and high pass filter that separates the two bands without the need to match-terminate the other. Diplexers are key components employed in the front-ends of microwave and mm-wave UWB measurements. Available diplexers at mm-wave frequencies up to $\mathrm{V}$ band have been reported before [ $\left.\mathrm{RSS}^{+} 09\right]$. However, they use suspended stripline topologies.

The diplexer presented here was designed on a 5 mil Rogers 3003 substrate for a low-cost microstrip topology. The contiguous diplexer with a crossover frequency of $50 \mathrm{GHz}$ was designed using a 6-section binomial design for the low-pass and high-pass filters. The diplexer circuit model is depicted in Fig. 4.30.

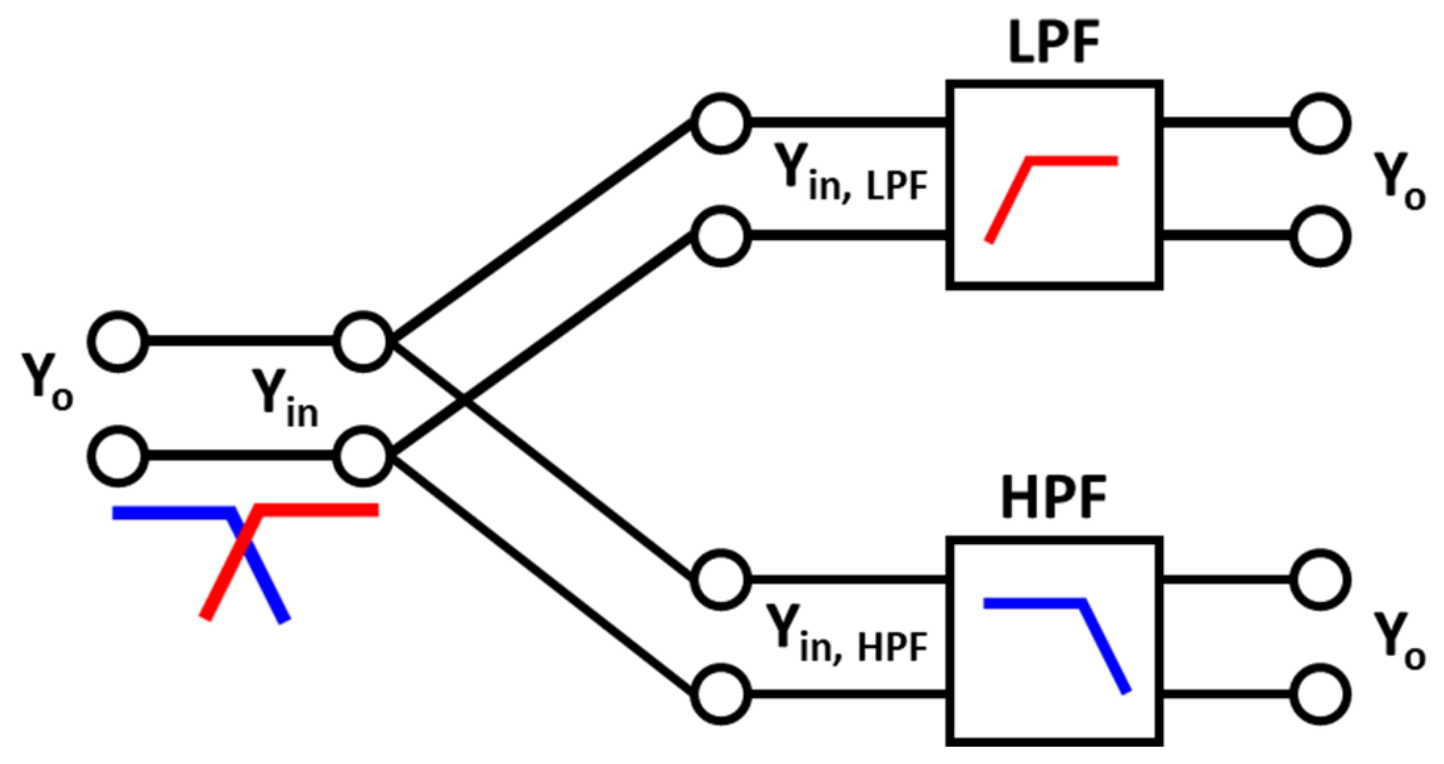

Figure 4.30: $50 \mathrm{GHz}$ split diplexer circuit model. 
Electric field patterns for the optimized design are given in Fig. 4.31(left) for the low pass filter. The middle of Fig. 4.31 shows the field patterns at the $50 \mathrm{GHz}$ crossover point and the right section of Fig. 4.31 refers to the high pass filter. Fig. 4.32 gives the simulated $S_{11}$ and $S_{21}$ data of the diplexer. As seen, every port is matched in its band of interest. Further, the diplexer shows a maximum insertion loss of $<0.5 \mathrm{~dB}$ in the low pass $(20 \mathrm{GHz}$ to $50 \mathrm{GHz})$ and $<1 \mathrm{~dB}$ in the high pass (above $50 \mathrm{GHz}$ ).

$20 \mathrm{GHz}$

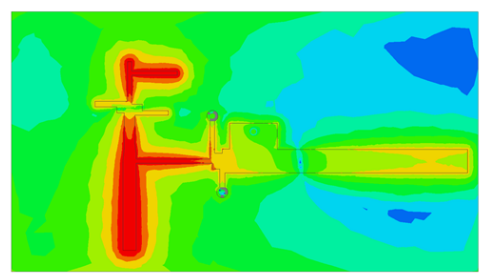

$50 \mathrm{GHz}$

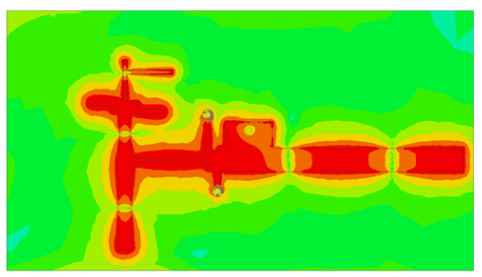

$80 \mathrm{GHz}$

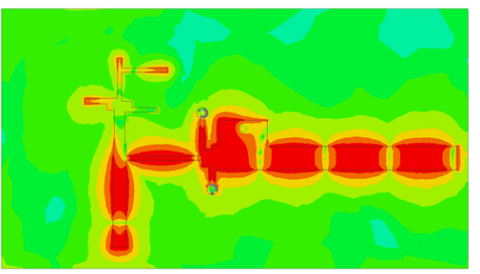

Figure 4.31: Electric field patterns for the diplexer at $20 \mathrm{GHz}$ (left), $50 \mathrm{GHz}$ (middle), and $80 \mathrm{GHz}$ (right).

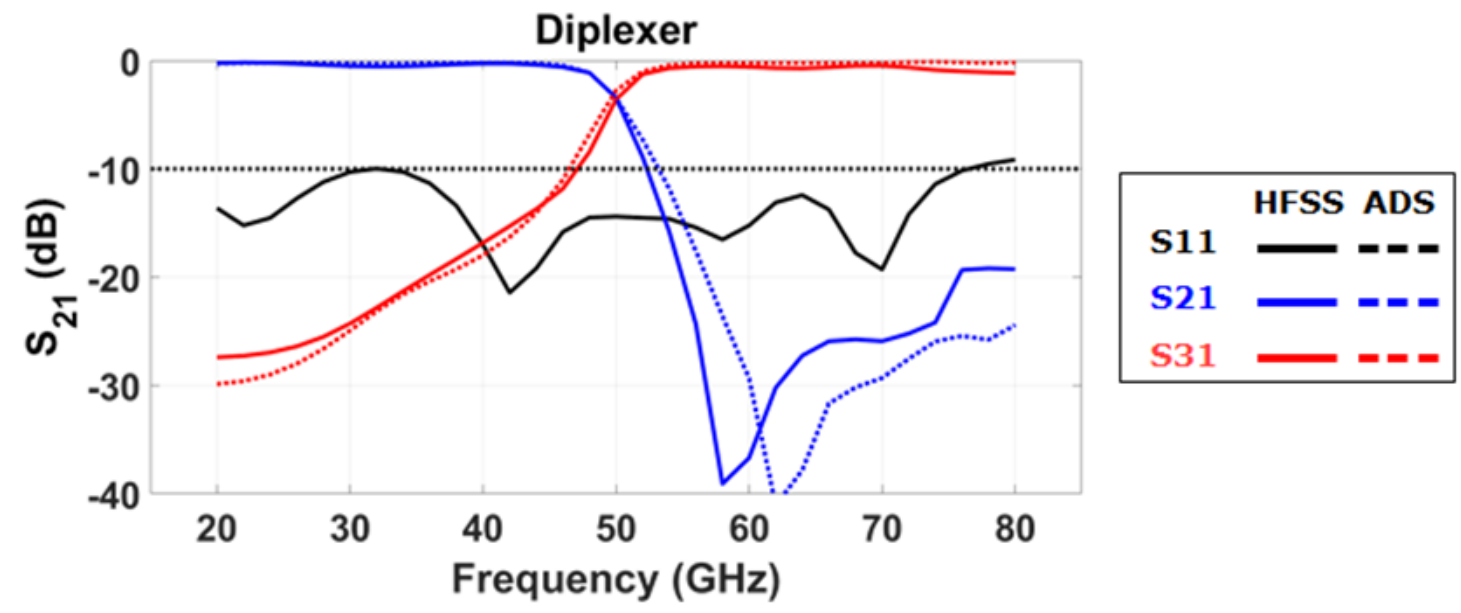

Figure 4.32: Simulated S-parameters for the $50 \mathrm{GHz}$ split diplexer. 


\section{Continuous UWB Feed}

The third and most convenient feeding approach is to employ an UWB $1.0 \mathrm{~mm}$ coax connector. However, this approach requires the same waveguides as before, with several coax-waveguide adapters. This implementation follows the simplicity of the Ka-band feed discussed above. Again, a via drives the array element's signal down to the back side of the feed structure, then the microstrip signal is directed under the ground plane to another via that leads up to the top side of the feed and into the $1.0 \mathrm{~mm}$ launcher. The simulations are given in Fig. 4.33 and Fig. 4.34.
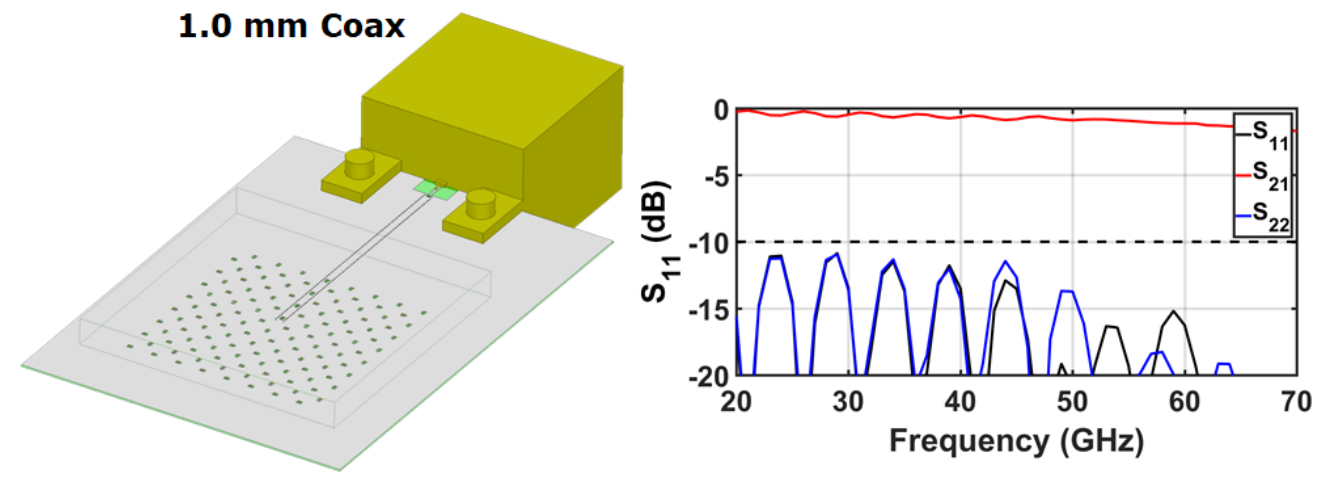

Figure 4.33: UWB feed structure for V-band balun-fed TCDA with $1.0 \mathrm{~mm}$ connector.
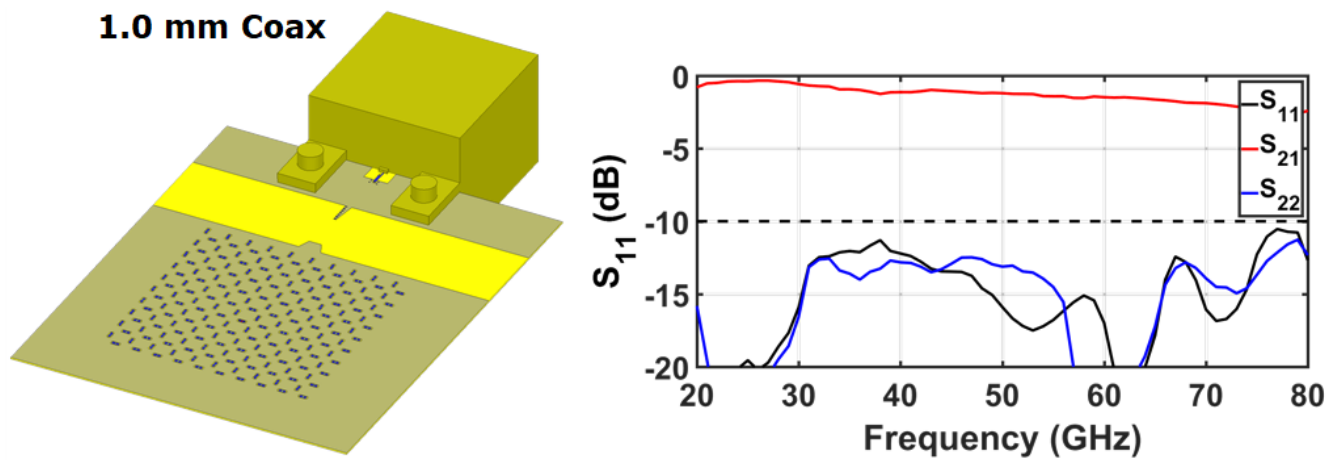

Figure 4.34: UWB balun feed structure for V-band differential TCDA with $1.0 \mathrm{~mm}$ connector. 


\subsubsection{De-embedding Process}

To determine the performance of measured array prototypes, the effects of the above mentioned feeds can be de-embedded using the test pieces in Fig. 4.36. Specifically, the losses of the feed boards can be measured by placing the feeds in a back to back orientation and measuring the $S_{21}$ of the test piece, as depicted in Fig. 4.35. From this measurement, since the feeds are passive and reciprocal, the loss of the feed boards can be de-embedded from the antenna performance.

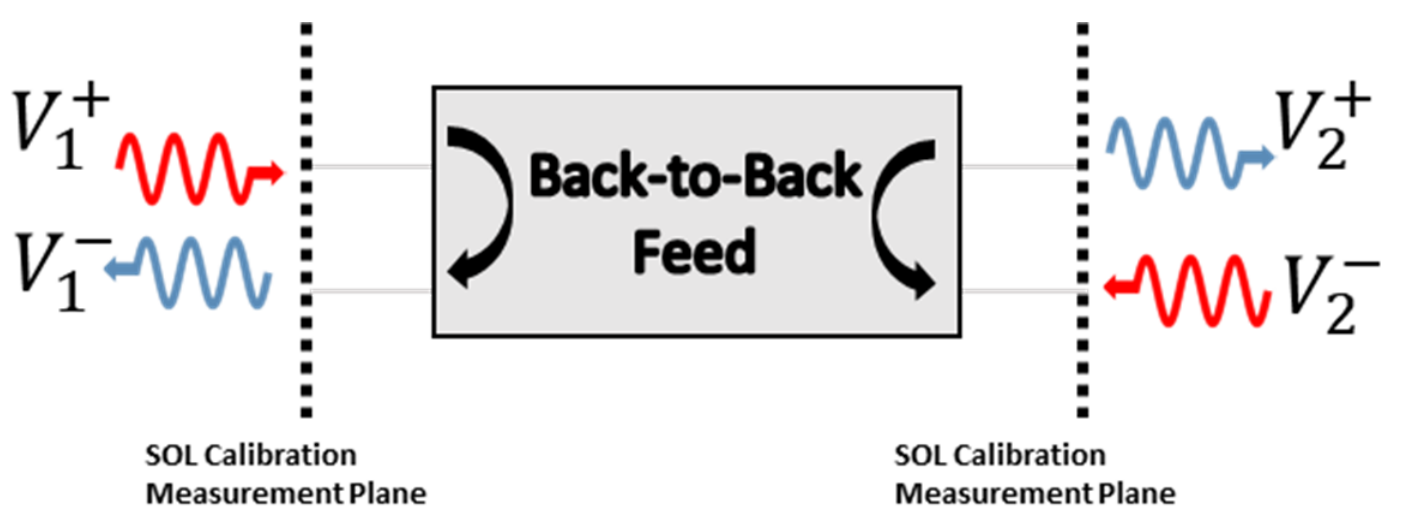

Figure 4.35: Back-to-back test piece de-embedding.

\section{Back to Back Power Combiner}
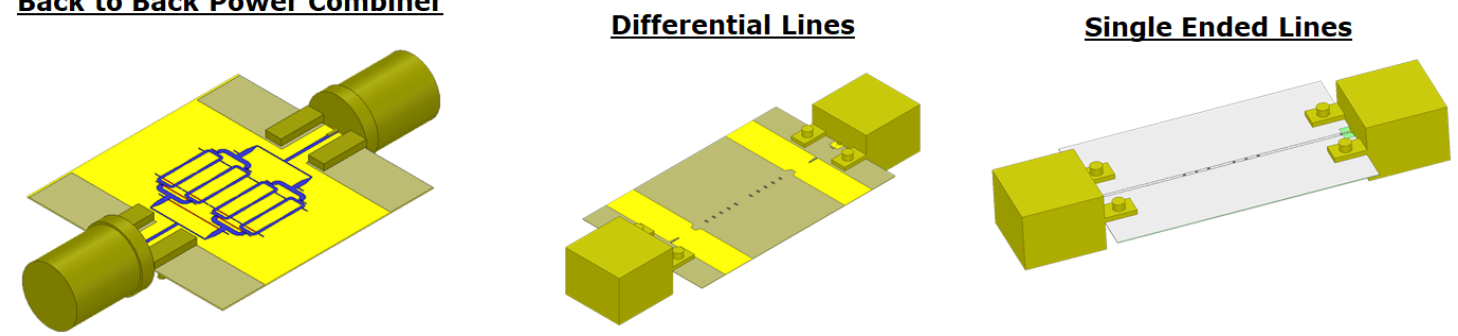

Figure 4.36: Test structures for de-embedding the losses from the antenna array feeds. 


\subsection{Link Budget Analysis and Communication Range}

Of course, with such an UWB instantaneous bandwidth, it begs the question of how to efficiently utilize multiple $\mathrm{GHz}$ of spectrum. With this single aperture $5 \mathrm{G}$ solution, one large array can be subdivided into sub-arrays for specific multi-band mm-wave applications, including: vehicle to vehicle (V2V) communications at 28 $\mathrm{GHz}$ [GVA18], the open-source ISM band at $60 \mathrm{GHz}$, and vehicular radar at 77 $\mathrm{GHz}$.

Through the use of sub-arrays and diplexers like those presented above, we can use the novel receiver architecture presented in [SAV18] to accommodate multiple signals, each with multi-GHz of instantaneous bandwidth, through a frequency division multiplexing front-end. Also, at the analog level, a beamforming architecture with hardware reduction $\left[\mathrm{VPA}^{+} 17\right]$ can be implemented to greatly reduce back-end power and size requirements. The total number of antenna elements can be optimized by conducting link budget analysis for specific frequencies of interest. For the example in Table I, a 10x10 array is used, assuming a transmit power of $30 \mathrm{dBm}$. The total gain is deduced from the $10 \times 1$ finite array simulated value, plus an array factor of $10 \log _{10}(10)$, or $10 \mathrm{~dB}$. For the case of $28 \mathrm{GHz}$ for $\mathrm{V} 2 \mathrm{~V}$ communication, it will allow reliable communication up to $9 \mathrm{~km}$. This is derived using the Friis transmission formula, with the assumption of 200 Megabits/second (Mbps) data rate, $E_{b} / N_{o}=10 \mathrm{~dB}$ (Energy per bit/noise spectral density) at the receiver, and a margin of $7 \mathrm{~dB}$. Notably, we also include the atmospheric losses at these frequencies, a well-known impediment to long distance mm-wave radio links. To improve communication range, we can easily scale the array size at the transmitter and receiver, by increasing sub-array size. To verify this link budget, two $28 \mathrm{GHz}$ reference horns were measured at a distance of 2.2 meters, as depicted in Fig. 4.37(a). The 
measured results in Fig. 4.37(b) are verified by the link budget in Table 4.4, with $P_{r x}=-18.2 \mathrm{dBm}$. By simply replacing the reference horns with the fabricated $10 \times 10 \mathrm{~mm}$-wave array we can demonstrate the link.

Table 4.4: Link budget

\begin{tabular}{|c|c|c|c|c|}
\hline Frequency $(\mathbf{G H z})$ & 28 & 28 & 60 & 77 \\
\hline Antenna Sub-array & Ref Horn & $10 \times 10$ & $10 \times 10$ & $10 \times 10$ \\
\hline Transmit Power $(\mathbf{d B m})$ & 10 & 30 & 30 & 30 \\
\hline$G_{r} \mid G_{t}(\mathbf{d B i})$ & $25 \mid 15$ & 15.7 & 21.2 & 23.9 \\
\hline Noise Figure $(\mathbf{d B})$ & 7 & 7 & 7 & 7 \\
\hline System Temp. $(\mathbf{d B}-\mathbf{K})$ & 31 & 31 & 31 & 31 \\
\hline Data Rate $(\mathbf{M b p s})$ & 200 & 200 & 200 & 200 \\
\hline Required $E_{b} / N_{o}(\mathrm{~dB})$ & 10 & 10 & 10 & 10 \\
\hline Modulation $(\mathbf{Q A M})$ & 16 & 16 & 16 & 16 \\
\hline Link Margin $(\mathbf{d B})$ & 7 & 7 & 7 & 7 \\
\hline Atmospheric Loss $(\mathbf{d B} / \mathbf{k m})$ & 0.12 & 0.12 & 14 & 0.4 \\
\hline Range $(\mathrm{km})$ & 0.0022 & 9 & 1.5 & 60.5 \\
\hline Received Power $(\mathbf{d B m})$ & -18.2 & -77 & -77 & -77 \\
\hline
\end{tabular}

Free Space Link Budget Analysis at 28GHz
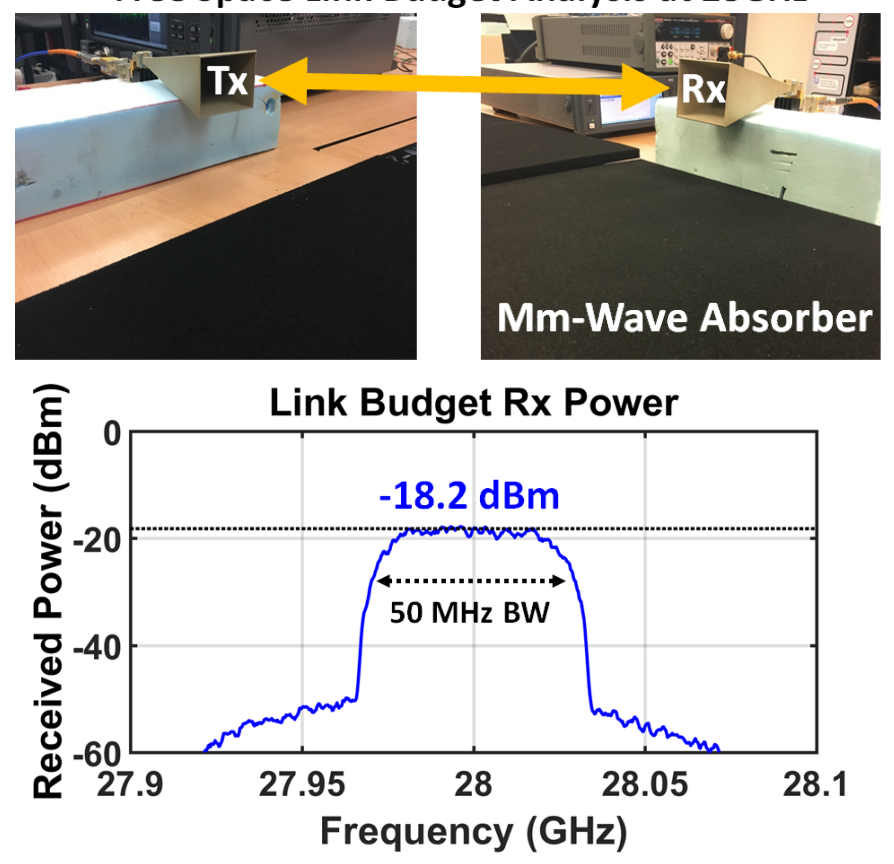

Figure 4.37: Link budget measurement using two $28 \mathrm{GHz}$ reference horns. 


\section{CHAPTER 5 \\ CONCLUSIONS AND FUTURE WORK}

\subsection{Summary of This Work}

The need for wideband antenna systems is driven by the simultaneity of an everexpanding need for bandwidth, continually shrinking platforms, and increasingly multi-functional systems. These qualities are observed throughout the defense, scientific, and consumer-electronic sectors, where ultra-wideband (UWB) phased arrays provide a highly effective solution to these modern challenges. For these systems, the ultra-wideband arrays presented here can replace several narrowband systems to reduce power, cost, and save space by leveraging time, frequency, and spatial multiplexing. Previous coupled dipole arrays have followed Munk's original work with extended bandwidths, $2 \times$ reduced size, $5 \times$ weight reduction, and $10 \times$

cost reduction. The TCDA presented here have been addressed and optimized to increase the impedance bandwidth to $>50: 1$ by substrate loading, scanning down to $75^{\circ}$ through FSS superstrates, and increased frequency operations in millimeterwaves. As a result, the TCDAs presented here can continuously cover a $130 \mathrm{MHz}$ to $80 \mathrm{GHz}$ bandwidth, as verified by measurements. Further, the weight reductions of these new TCDA, as given in Table 5.1 have a great impact for mobile platforms. The summary of this dissertation is as follows.

In Chapter 2 we outlined the development and measurement of three state-ofthe-art UWB TCDA. The first TCDA presented herein obtained the 9:1 loss-less bandwidth limit with $60^{\circ}$ scanning. The second TCDA used resistive loading to surpass this limit for a 12:1 bandwidth with $60^{\circ}$ scanning for the first time, and was the first TCDA to be designed with the specific needs of an airborne platform. The 
Table 5.1: Weight Comparison of Past and presented TCDA

\begin{tabular}{|c|c|c|c|c|c|}
\hline Reference & Pol & BW Ratio & Frequency (GHz) & Scanning & Mass (kg) \\
\hline [MSV13] & Single & $13.9: 1$ & $0.29-3.9$ & $45^{\circ}$ & $>5$ \\
\hline [PV16a] & Dual & $13.1: 1$ & $0.3-4.47$ & $45^{\circ}$ & 2.442 \\
\hline Chapt. 2.1 & Dual & $9: 1$ & $2-18$ & $60^{\circ}$ & 0.351 \\
\hline Chapt. 2.2 & Dual & $12: 1$ & $0.19-2.3$ & $60^{\circ}$ & 6.577 \\
\hline Chapt. 2.3 & Dual & $58: 1$ & $0.13-7.6$ & $60^{\circ}$ & 3.012 \\
\hline Chapt. 3.1 & Dual & $8.5: 1$ & $0.4-3.2$ & $45^{\circ}$ & 1.943 \\
\hline Chapt. 3.2 & Dual & $6: 1$ & $3-18$ & $45^{\circ}$ & - \\
\hline Chapt. 4.2 & Dual & $7.5: 1$ & $4-30$ & $60^{\circ}$ & $<0.001$ \\
\hline Chapt. 4.2 & Dual & $3.3: 1$ & $21-70$ & $60^{\circ}$ & $<0.001$ \\
\hline Chapt. 4.3 & Dual & $3.63: 1$ & $22-80$ & $60^{\circ}$ & $<0.001$ \\
\hline
\end{tabular}

third TCDA introduced a ground-breaking resistive FSS and novel feed that enables a $58: 1$ bandwidth broadside with $60^{\circ}$ conical scanning.

In Chapter 3, we presented the development and measurement of a novel TCDA with differential feed. This is the first UWB differential array to scan to low angles without resonances in the E-Plane over an unprecedented 8.5:1 bandwidth. The design and operation of the enabling Balanced Wideband Impedance Transformer (BWIT) feed structure was discussed. This array achieved an ultra-wide band performance from $\mathrm{V}$, with scanning to $45^{\circ}$. Polarization isolation was kept at an average of $60 \mathrm{~dB}$ at broadside and at $40 \mathrm{~dB}$ when scanning in E- and H-planes. A Ku band D-TCDA was presented for operation over 3-19 GHz, to show the scalability of the array and feed BWIT structure.

Chapter 4 outlines the development and measurement of four mm-wave TCDA with active array feeding. The first two UWB array were focused on low cost manufacturing, with single board PCB construction in two important mm-wave bands. The first array achieved an ultra-wide band performance from $20 \mathrm{GHz}$ to $65 \mathrm{GHz}$, with scanning to $60^{\circ}$. The second array achieved an ultra-wide band performance from $4 \mathrm{GHz}$ to $30 \mathrm{GHz}$, with scanning to $60^{\circ}$. For both designs, polarization isola- 
tion was kept at an average of $60 \mathrm{~dB}$ at broadside and at $40 \mathrm{~dB}$ when scanning in E-planes and H-planes. This third UWB array was focused on low cost manufacturing with differential feeding. The differential array achieved an ultra-wide band performance from $22 \mathrm{GHz}$ to $80 \mathrm{GHz}$, with scanning to $60^{\circ}$. Polarization isolation was kept at an average of $60 \mathrm{~dB}$ at broadside and at $40 \mathrm{~dB}$ when scanning in E- and H-planes. Again, a novel feed structure, denoted as an $\mathrm{H}$ - $\mathrm{Wall}$ enables $60^{\circ}$ scanning on low cost PCB. The three aforementioned arrays were designed with integration via BGA solder balls in mind. Measurements are provided for a fourth array using a novel UWB feed routing network using vias, designed from $25 \mathrm{GHz}$ to $80 \mathrm{GHz}$ with a return loss of less than $-10 \mathrm{~dB}$. The total cost of this fabricated array was 50 cents per element.

\subsection{New Work}

\subsubsection{Fabrication and Measurements of mm-wave Arrays}

For future measurements, the differential array was redesigned using Rogers 3003 material. By switching from the Rogers 5880 material $\left(\epsilon_{r}=2.2\right)$ to the Rogers 3003 material $\left(\epsilon_{r}=3.0\right)$ the array was able lower profile to comply with standard aspect ratio requirements. This allows for direct via integration with an integrated feed board using 4 mil vias, rather than BGA, for a first-look verification of the design. This array, depicted in Fig. 5.1 has a slightly reduced bandwidth of $25-80 \mathrm{GHz}$, but also a lower profile of $1.17 \mathrm{~mm}$. The $1.0 \mathrm{~mm}$ coax connector feed will be used to excite this array. Notably, any misalignment between the coaxial connector and feed line can have a great effect on insertion losses [Nov17]. Measurements of this array will be done in follow-up works. 


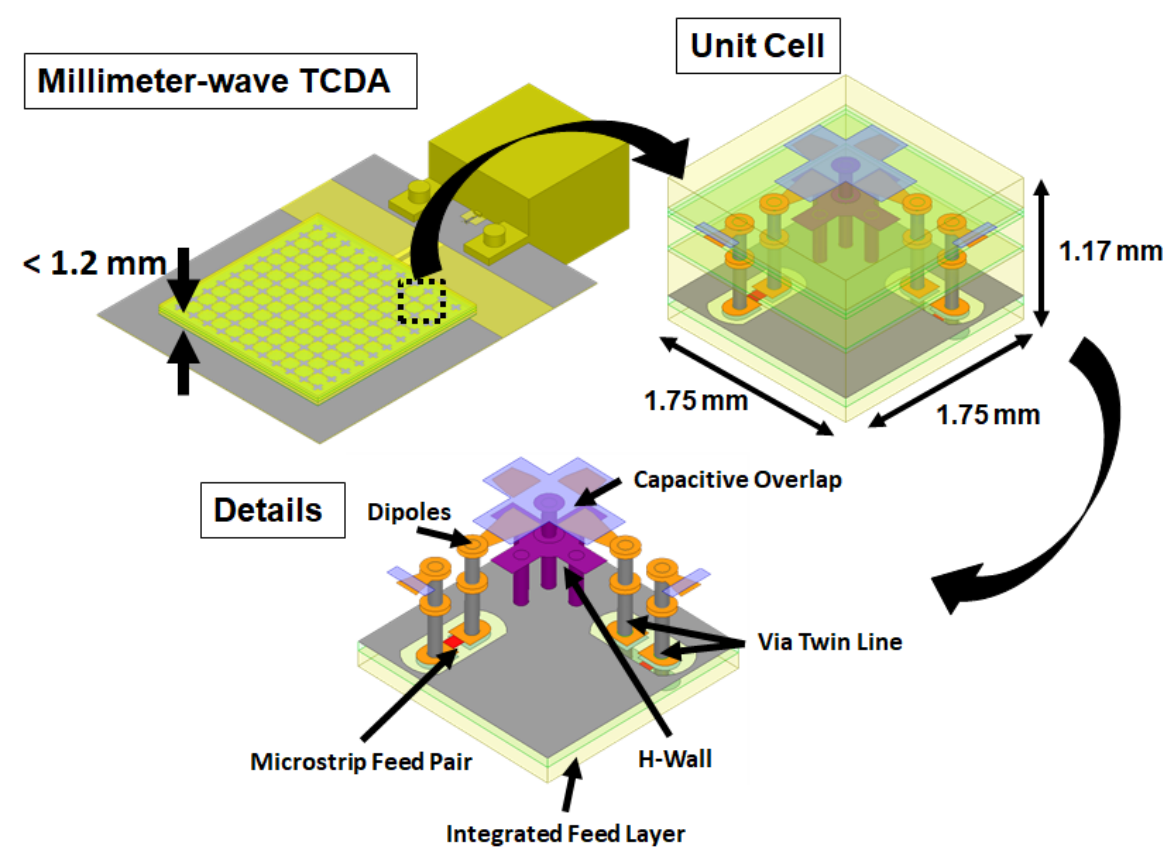

Figure 5.1: Pictorial representation of the fabricated differential TCDA array including an enlarged view of the unit cell with integrated feed board.

\subsubsection{Aperture in Aperture}

Following work will consist of integrating the aforementioned arrays to cover a continuous $>500: 1$ bandwidth, with low angle scanning, through the use of sub-arrays. This Aperture in Aperture (AIA) concept, depicted in Fig. 5.2 will enable communication and sensing capabilities across almost all of the available radio spectrum. For advanced communications and Electronic Warfare (EW) systems systems, this UWB array replaces several narrowband systems for orders of magnitude power, cost, and space savings. It also enables increased data rates and secure spread spectrum communications over the UHF-V bands. 

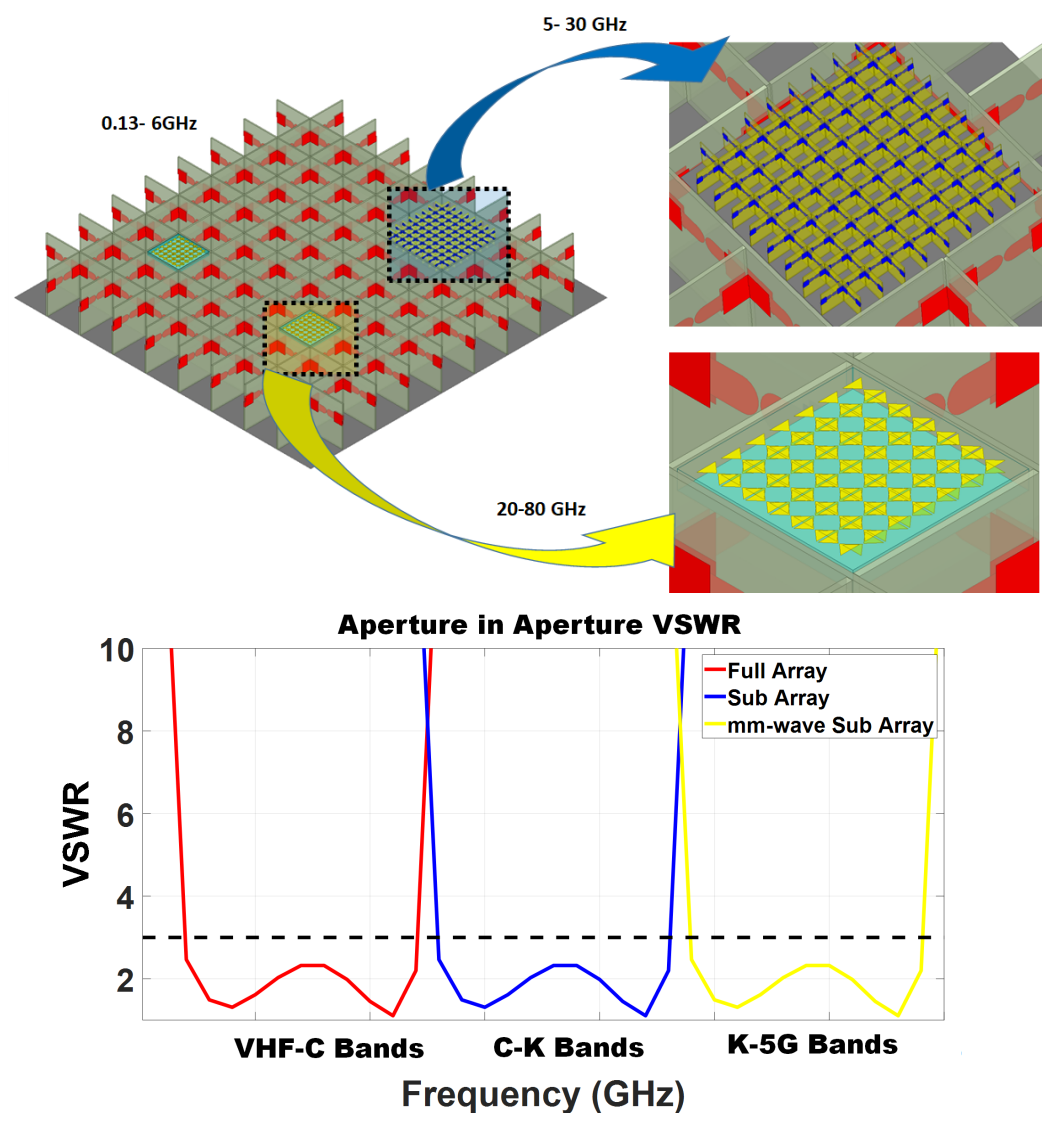

Figure 5.2: Pictorial representation of the Aperture in Aperture concept.

\subsubsection{Novel loading for TCDA with Extended Bandwidths and Lower Profiles}

Following the work on extending bandwidths, novel loading techniques will be explored. These include loading through magnetic ground planes (See Fig. 5.3(a)), EBG ground planes (see Fig. 5.3(b)), and non-resistive FSS substrate loading (see Fig. 5.3(c)). First look simulations have been conducted, with results given in Fig. $5.3(\mathrm{~d})$. 


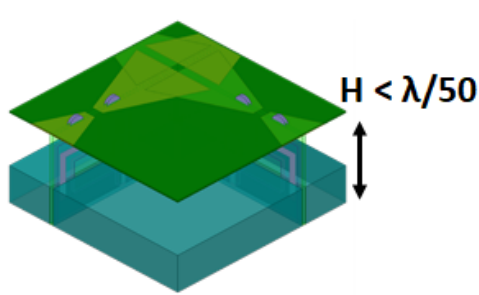

(a)

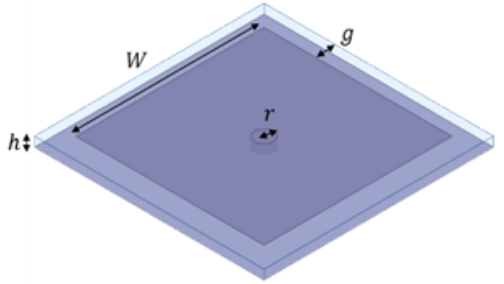

(b)

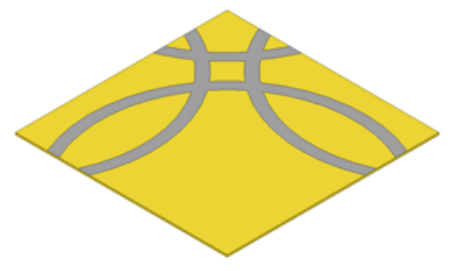

(c)

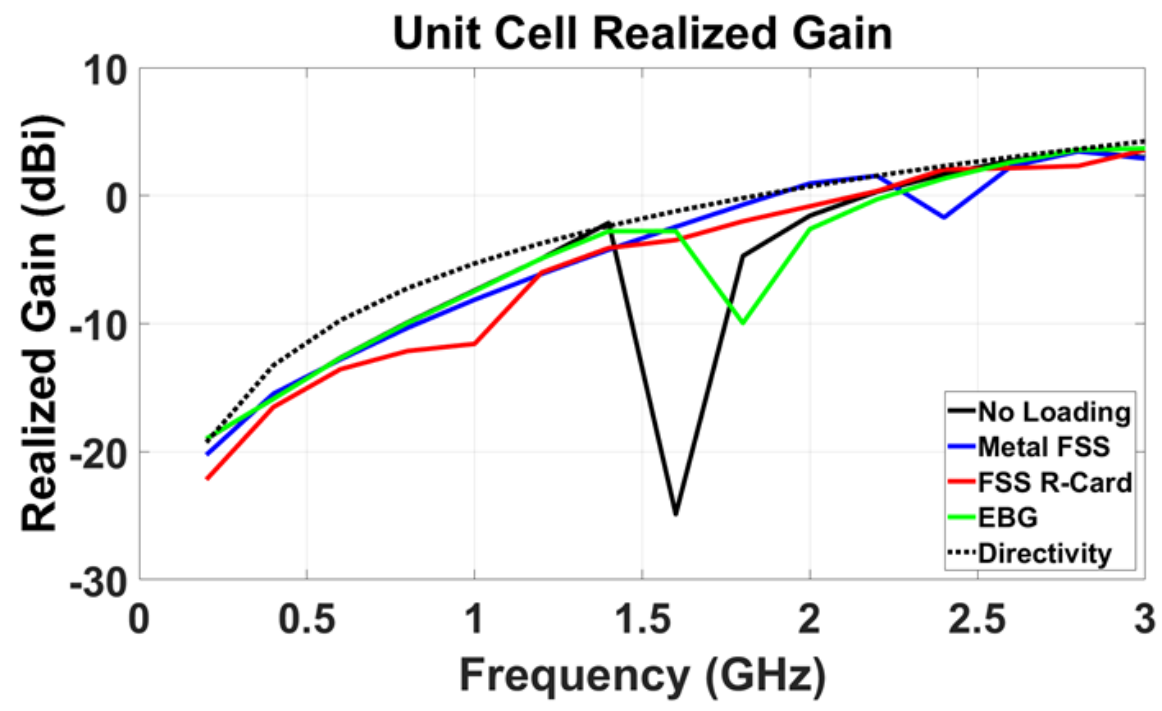

(d)

Figure 5.3: Pictorial representation novel loading techniques for TCDA using (a) magnetic loading, (b) EBG ground planes, and (c) metal FSS substrates. (d) A comparison of these methods versus the designs shown here is given 


\section{BIBLIOGRAPHY}

[588] Rt/duroid 5880lz datasheet.

$\left[\mathrm{ACP}^{+} 13\right] \quad$ A. L. Amadjikpe, D. Choudhury, C. E. Patterson, B. Lacroix, G. E. Ponchak, and J. Papapolymerou. Integrated 60-ghz antenna on multilayer organic package with broadside and end-fire radiation. IEEE Transactions on Microwave Theory and Techniques, 61(1):303-315, Jan 2013.

$\left[\mathrm{AJM}^{+} 17\right] \quad$ S. Adamshick, A. Johnson, K. Moriarty, W. Tremblay, and J. Burke. Antenna on chip design utilizing 3D integration for mixed signal applications. In 2017 IEEE 60th International Midwest Symposium on Circuits and Systems (MWSCAS), pages 209-212, Aug 2017.

[ANHS17] A. J. Abumunshar, N. K. Nahar, D. Hyman, and K. Sertel. $18-40$ ghz low-profile phased array with integrated mems phase shifters. In 2017 11th European Conference on Antennas and Propagation (EUCAP), pages 2800-2801, March 2017.

[ASV12] E. A. Alwan, K. Sertel, and J. L. Volakis. Circuit model based optimization of ultra-wideband arrays. In Proceedings of the 2012 IEEE International Symposium on Antennas and Propagation, pages 1-2, July 2012 .

[Bal08] C. A. Balanis. Wideband Arrays. Wiley, 2008.

$\left[\mathrm{BAP}^{+} 12\right] \quad$ A. Barakat, A. Allam, R. K. Pokharel, H. Elsadek, M. El-Sayed, and K. Yoshida. Performance optimization of a 60 ghz antennaon-chip over an artificial magnetic conductor. In 2012 Japan-Egypt Conference on Electronics, Communications and Computers, pages 118-121, March 2012.

[BF07] M. Buck and D. Filipovic. Bi-layer, mode 2, four-arm spiral antennas. Electronics Letters, 43(6):1-2, March 2007.

[BG16] A. Boryssenko and T. Goodwin. Broadband antenna array aperture made of tightly couple printed dipoles. In 2016 IEEE International Symposium on Phased Array Systems and Technology (PAST), pages 1-6, Oct 2016. 
[BMIM16] T. S. Beukman, P. Meyer, M. V. Ivashina, and R. Maaskant. Modalbased design of a wideband quadruple-ridged flared horn antenna. IEEE Transactions on Antennas and Propagation, 64(5):1615-1626, May 2016.

[BQG16] A. O. Bah, Pei-Yuan Qin, and Y. J. Guo. An extremely wideband tapered balun for application in tightly coupled arrays. In 2016 IEEE-APS Topical Conference on Antennas and Propagation in Wireless Communications (APWC), pages 162-165, Sept 2016.

[BQZ $\left.{ }^{+} 19\right] \quad$ A. O. Bah, P. Qin, R. W. Ziolkowski, Y. J. Guo, and T. S. Bird. A wideband low-profile tightly coupled antenna array with a very high figure of merit. IEEE Transactions on Antennas and Propagation, pages 1-1, 2019.

[BSC93] J. R. Bayard, D. H. Schaubert, and M. E. Cooley. E-plane scan performance of infinite arrays of dipoles printed on protruding dielectric substrates: coplanar feed line and e-plane metallic wall effects. IEEE Transactions on Antennas and Propagation, 41(6):837-841, June 1993.

[Chu48] L. J. Chu. Physical limitations of omnidirectional antennas. Journal of Applied Physics, 19(12):1163-1175, 1948.

[CK16] H. Chang and D. Kwon. Higher-order bandwidth bounds for conductor-backed planar arrays. In 2016 IEEE International Symposium on Antennas and Propagation (APSURSI), pages 921-922, June 2016.

[CSN17] D. Cavallo, W. H. Syed, and A. Neto. Equivalent transmission line models for the analysis of edge effects in finite connected and tightly coupled arrays. IEEE Transactions on Antennas and Propagation, 65(4):1788-1796, April 2017.

[CSN18] D. Cavallo, W. H. Syed, and A. Neto. Connected-slot array with artificial dielectrics: A 6 to 15 ghz dual-pol wide-scan prototype. IEEE Transactions on Antennas and Propagation, 66(6):3201-3206, June 2018.

[Dir14] IEEE Standard 145-2013 (Revision of IEEE Standard 145-1993), pages 1-50. IEEE, March 2014. 
[dLAGGP $\left.{ }^{+} 10\right]$ E. de Lera Acedo, E. Garcia, V. Gonzlez-Posadas, J. L. VazquezRoy, R. Maaskant, and D. Segovia. Study and design of a differentially-fed tapered slot antenna array. IEEE Transactions on Antennas and Propagation, 58(1):68-78, Jan 2010.

$\left[\mathrm{dRG}^{+} 11\right] \quad$ E. de Lera Acedo, N. Razavi-Ghods, L. E. Garcia, P. Duffett-Smith, and P. Alexander. Ultra-Wideband Aperture Array Element Design for Low Frequency Radio Astronomy. IEEE Transactions on Antennas and Propagation, 59(6):1808-1816, June 2011.

[DSV12] J. P. Doane, K. Sertel, and J. L. Volakis. Bandwidth limits for lossless planar arrays over ground plane. Electronics Letters, 48(10):540-542, May 2012.

[DSV13a] J. P. Doane, K. Sertel, and J. L. Volakis. Matching bandwidth limits for arrays backed by a conducting ground plane. IEEE Transactions on Antennas and Propagation, 61(5):2511-2518, May 2013.

[DSV13b] J. P. Doane, K. Sertel, and J. L. Volakis. Matching Bandwidth Limits for Arrays Backed by a Conducting Ground Plane. IEEE Transactions on Antennas and Propagation, 61(5):2511-2518, May 2013.

[DSV13c] J. P. Doane, K. Sertel, and J. L. Volakis. A wideband, wide scanning tightly coupled dipole array with integrated balun (tcda-ib). IEEE Transactions on Antennas and Propagation, 61(9):4538-4548, Sept 2013.

[DSV14] J. P. Doane, K. Sertel, and J. L. Volakis. Bandwidth limits for lossless, reciprocal pec-backed arrays of arbitrary polarization. IEEE Transactions on Antennas and Propagation, 62(5):2531-2542, May 2014.

[EM11] M. W. Elsallal and J. C. Mather. An ultra-thin, decade (10:1) bandwidth, modular bava array with low cross-polarization. In 2011 IEEE International Symposium on Antennas and Propagation (APSURSI), pages 1980-1983, July 2011.

[EO60] S. Edelberg and A. Oliner. Mutual Coupling Effects in Large Antenna Arrays II: Compensation Effects. IRE Transactions on Antennas and Propagation, 8(4):360-367, July 1960. 
[FAZ $\left.{ }^{+} 13\right] \quad$ E O Farhat, K Z Adami, Y Zhang, A K Brown, and C V Sammut. Ultra-Wideband Tightly Coupled Phased Array Antenna for LowFrequency Radio Telescope. In PIERS Proceedings Stockholm, pages 245-249, 2013.

[GVA18] S. R. Govindarajulu, S. B. Venkatakrishnan, and E. A. Alwan. Millimeter-Wave Wideband Array for Vehicle to Vehicle Communication. In 2018 IEEE International Symposium on Antennas and Propagation USNC/URSI National Radio Science Meeting, pages 1203-1204, July 2018.

[HBG13] W. Hong, K. Baek, and A. Goudelev. Grid assembly-free 60-ghz antenna module embedded in fr-4 transceiver carrier board. IEEE Transactions on Antennas and Propagation, 61(4):1573-1580, April 2013.

[HDGV99] C. Hemmi, R. T. Dover, F. German, and A. Vespa. Multifunction wide-band array design. IEEE Transactions on Antennas and Propagation, 47(3):425-431, March 1999.

[Hol11] S.S Holland. Low-profile, modular, ultra-wideband phased arrays. $\mathrm{PhD}$ thesis, Ph.D. dissertation, Dept. Elect. Comp. Eng., Univ. Massachusetts, Amherst, MA, USA, 2011.

[HSV12] S. S. Holland, D. H. Schaubert, and M. N. Vouvakis. A $7-21 \mathrm{ghz}$ dual-polarized planar ultrawideband modular antenna (puma) array. IEEE Transactions on Antennas and Propagation, 60(10):45894600, Oct 2012.

[HV10] S. S. Holland and M. N. Vouvakis. A $7-21$ ghz planar ultrawideband modular array. In 2010 IEEE Antennas and Propagation Society International Symposium, pages 1-4, July 2010.

[HV11] S. S. Holland and M. N. Vouvakis. The Banyan Tree Antenna Array. IEEE Transactions on Antennas and Propagation, 59(11):40604070, Nov 2011.

[HV12] S. S. Holland and M. N. Vouvakis. The planar ultrawideband modular antenna (puma) array. IEEE Transactions on Antennas and Propagation, 60(1):130-140, Jan 2012. 
[Isb60] D. Isbell. Log periodic dipole arrays. IRE Transactions on Antennas and Propagation, 8(3):260-267, May 1960.

[JDRGHN ${ }^{+}$18] Brock J. DeLong, Sandhiya Reddy Govindarajulu, Markus H. Novak, Elias Alwan, and John L. Volakis. A $60 \mathrm{GHz}$ phased array with measurement and de-embedding techniques. Analog Integrated Circuits and Signal Processing, 97, 082018.

[JR07] M. Jones and J. Rawnick. A new approach to broadband array design using tightly coupled elements. In MILCOM 2007 - IEEE Military Communications Conference, pages 1-7, Oct 2007.

$\left[\mathrm{JSN}^{+} 18\right] \quad$ J. W. Jordan, W. Stacy, J. Ng, B. L. Cannon, A. Caba, K. J. Vanhille, J. Clough, B. Stant, and P. E. Racette. Polystrata x/ku/k/kaband, dual-polarized, tightly coupled dipole scannable focal plane array. In 2018 IEEE International Symposium on Antennas and Propagation USNC/URSI National Radio Science Meeting, pages 817-818, July 2018.

[KCV13] J. A. Kasemodel, C. C. Chen, and J. L. Volakis. Wideband planar array with integrated feed and matching network for wide-angle scanning. IEEE Transactions on Antennas and Propagation, 61(9):45284537, 92013.

[KKYY18] T. Kim, Y. Kim, T. Yoo, and J. Yook. Wideband planar monopole antenna for digital TV reception and UHF band communications. IET Microwaves, Antennas Propagation, 12(13):2041-2045, 2018.

$\left[\mathrm{KLN}^{+} 11\right] \quad$ D. G. Kam, D. Liu, A. Natarajan, S. Reynolds, H. Chen, and B. A. Floyd. Ltcc packages with embedded phased-array antennas for 60 ghz communications. IEEE Microwave and Wireless Components Letters, 21(3):142-144, March 2011.

[KRS $\left.{ }^{+} 18\right]$ M. R. Konkol, D. D. Ross, S. Shi, C. E. Harrity, A. A. Wright, C. A. Schuetz, and D. W. Prather. Photonic tightly coupled array. IEEE Transactions on Microwave Theory and Techniques, 66(5):2570-2578, May 2018.

[LCQ14] W. Liu, Z. N. Chen, and X. Qing. 60-ghz thin broadband high-gain ltcc metamaterial-mushroom antenna array. IEEE Transactions on Antennas and Propagation, 62(9):4592-4601, Sep. 2014. 
[LGH $\left.{ }^{+} 16\right] \quad$ X. Li, C. Gu, G. Han, Z. Zhou, S. Lu, and Z. Li. A uwb wide-scan tightly coupled dipole array. In 2016 IEEE International Workshop on Electromagnetics: Applications and Student Innovation Competition (iWEM), pages 1-3, May 2016.

[LGZ $\left.{ }^{+} 18\right] \quad$ W. Li, S. Gao, L. Zhang, Q. Luo, and Y. Cai. An ultra-wide-band tightly coupled dipole reflectarray antenna. IEEE Transactions on Antennas and Propagation, 66(2):533-540, Feb 2018.

[LKLV18] J. T. Logan, R. W. Kindt, M. Y. Lee, and M. N. Vouvakis. A new class of planar ultrawideband modular antenna arrays with improved bandwidth. IEEE Transactions on Antennas and Propagation, 66(2):692-701, Feb 2018.

[LKV18] J. T. Logan, R. W. Kindt, and M. N. Vouvakis. A 1.2 to 12 ghz sliced notch antenna array. IEEE Transactions on Antennas and Propagation, 66(4):1818-1826, 42018.

[LL93] J. J. Lee and S. Livingston. Wide band bunny-ear radiating element. In Proceedings of IEEE Antennas and Propagation Society International Symposium, pages 1604-1607 vol.3, June 1993.

[LLJ $\left.{ }^{+} 19\right] \quad$ H. Lee, E. S. Li, H. Jin, C. Li, and K. Chin. $60 \mathrm{GHz}$ wideband LTCC microstrip patch antenna array with parasitic surrounding stacked patches. IET Microwaves, Antennas Propagation, 13(1):3541, 2019.

[LLK04] J. J. Lee, S. Livingston, and R. Koenig. Performance of a wideband (3-14 ghz) dual-pol array. In IEEE Antennas and Propagation Society Symposium, 2004., volume 1, pages 551-554 Vol.1, June 2004.

[LN10] Chieh-Ping Lai and Ram Narayanan. Ultrawideband Random Noise Radar Design for Through-Wall Surveillance. Aerospace and Electronic Systems, IEEE Transactions on, 46:1716 - 1730, 112010.

[Lud73] A. Ludwig. The definition of cross polarization. IEEE Transactions on Antennas and Propagation, 21(1):116-119, January 1973.

$\left[\mathrm{MBL}^{+} 11\right] \quad$ J. G. Maloney, B. N. Baker, R. T. Lee, G. N. Kiesel, and J. J. Acree. Wide scan, integrated printed circuit board, fragmented aperture array antennas. In 2011 IEEE International Symposium on Antennas and Propagation (APSURSI), pages 1965-1968, July 2011. 
[MKCS17] M. Moosazadeh, S. Kharkovsky, J. T. Case, and B. Samali. Improved radiation characteristics of small antipodal vivaldi antenna for microwave and millimeter-wave imaging applications. IEEE Antennas and Wireless Propagation Letters, 16:1961-1964, 2017.

[MSV12a] W. F. Moulder, K. Sertel, and J. L. Volakis. Superstrate-enhanced ultrawideband tightly coupled array with resistive fss. IEEE Transactions on Antennas and Propagation, 60(9):4166-4172, Sept 2012.

[MSV12b] W. F. Moulder, K. Sertel, and J. L. Volakis. Superstrate-enhanced ultrawideband tightly coupled array with resistive fss. IEEE Transactions on Antennas and Propagation, 60(9):4166-4172, Sept 2012.

[MSV13] W. F. Moulder, K. Sertel, and J. L. Volakis. Ultrawideband superstrate-enhanced substrate-loaded array with integrated feed. IEEE Transactions on Antennas and Propagation, 61(11):58025807, Nov 2013.

$\left[\mathrm{MTD}^{+}\right.$03] B. Munk, R. Taylor, T. Durharn, W. Croswell, B. Pigon, R. Boozer, S. Brown, M. Jones, J. Pryor, S. Ortiz, J. Rawnick, K. Krebs, M. Vanstrum, G. Gothard, and D. Wiebelt. A low-profile broadband phased array antenna. In IEEE Antennas and Propagation Society International Symposium. Digest. Held in conjunction with: USNC/CNC/URSI North American Radio Sci. Meeting (Cat. No.03CH37450), volume 2, pages 448-451 vol.2, June 2003.

[Mun03] Ben A. Munk. Broadband Wire Arrays, pages 181-213. Wiley-IEEE Press, 2003.

[Mun05] B.A. Munk. Frequency Selective Surfaces: Theory and Design. Wiley, 2005.

[MYG18] S. M. Moghaddam, J. Yang, and A. A. Glazunov. A planar dualpolarized ultra-wideband millimeter-wave array antenna. In 12th European Conference on Antennas and Propagation (EuCAP 2018), pages 1-3, April 2018.

[NAS15] Communication and Navigation Systems Roadmap. NASA, July 2015 .

[Nea99] J.R. Nealy. Foursquare antenna radiating element. U.S. Patent 5 926 137A, July 20, 1999. 
$\left[\mathrm{NEA}^{+} 11\right] \quad$ D. Nashaat, H. A. Elsadek, E. A. Abdallah, M. F. Iskander, and H. M. Elhenawy. Ultrawide Bandwidth $2 \times 2$ Microstrip Patch Array Antenna Using Electromagnetic Band-Gap Structure (EBG). IEEE Transactions on Antennas and Propagation, 59(5):1528-1534, May 2011.

[NGKS15] R. Natarajan, J. V. George, M. Kanagasabai, and A. Kumar Shrivastav. A compact antipodal vivaldi antenna for uwb applications. IEEE Antennas and Wireless Propagation Letters, 14:1557-1560, 2015.

[NMV17] M. H. Novak, F. A. Miranda, and J. L. Volakis. Ultra-wideband phased array for small satellite communications. IET Microwaves, Antennas Propagation, 11(9):1234-1240, 2017.

[NMV18] M. H. Novak, F. A. Miranda, and J. L. Volakis. Ultra-wideband phased array for millimeter-wave ism and $5 \mathrm{~g}$ bands, realized in pcb. IEEE Transactions on Antennas and Propagation, 66(12):69306938, Dec 2018.

[Nov17] Markus Novak. Low Cost Ultra-Wideband Millimeter-Wave Phased Arrays. PhD thesis, Ph.D. dissertation, Ohio State University, 2017.

[Poz94] D. M. Pozar. The active element pattern. IEEE Transactions on Antennas and Propagation, 42(8):1176-1178, Aug 1994.

[Poz12] D. M. Pozar. Microwave engineering education: From field theory to circuit theory. In 2012 IEEE/MTT-S International Microwave Symposium Digest, pages 1-3, 62012.

[PV16a] D. K. Papantonis and J. L. Volakis. Dual-polarized tightly coupled array with substrate loading. IEEE Antennas and Wireless Propagation Letters, 15:325-328, 2016.

[PV16b] D. K. Papantonis and J. L. Volakis. Tunable band rejection of wideband arrays using digital variable capacitors. In 2016 IEEE International Symposium on Antennas and Propagation (APSURSI), pages 647-648, 62016.

[PYV16] D. K. Papantonis, E. Yetisir, and J. L. Volakis. Tightly-coupled array with tunable bw using reconfigurable ffs/superstrate. In 2016 USNC-URSI Radio Science Meeting, pages 13-14, June 2016. 
[Rad07] Ieee standard for ultrawideband radar definitions. IEEE Std 16722006, pages c1-9, May 2007.

[RJB14] B. Riviere, H. Jeuland, and S. Bolioli. New equivalent circuit model for a broadband optimization of dipole arrays. IEEE Antennas and Wireless Propagation Letters, 13:1300-1304, 2014.

[RSS $\left.{ }^{+} 09\right]$ R. Rehner, M. Sterns, D. Schneiderbanger, S. Martius, and L. Schmidt. A quasi-lumped ultra-broadband contiguous SSLdiplexer from DC to $80 \mathrm{GHz}$. In 2009 IEEE MTT-S International Microwave Symposium Digest, pages 1037-1040, June 2009.

[S. 08] S. G. Hay and J. D. O'Sullivan. Analysis of Common-mode Effects in a Dual Polarized Planar Connected-Array Antenna. Radio Science RS6S04, 43, Dec 2008.

[SAV18] D. Siafarikas, E. A. Alwan, and J. L. Volakis. Interference Mitigation for 5G Millimeter-Wave Communications. In 2018 IEEE International Symposium on Antennas and Propagation USNC/URSI National Radio Science Meeting, pages 391-392, July 2018.

$\left[\mathrm{SBN}^{+} 15\right] \quad$ S. Shi, J. Bai, R. Nelson, C. Schuetz, P. Yao, G. J. Schneider, Y. Zhang, and D. W. Prather. Ultrawideband optically fed tightly coupled phased array. Journal of Lightwave Technology, 33(23):4781-4790, Dec 2015.

[SC16] A. A. Salih and Z. N. Chen. Excitation for tightly coupled beam scanning antenna array based on inverse scattering. In 2016 IEEE MTT-S International Conference on Numerical Electromagnetic and Multiphysics Modeling and Optimization (NEMO), pages 1-2, July 2016.

[SCNS18] S. Sahin, C. Caglayan, N. K. Nahar, and K. Sertel. Performance characterization of monolithically integrated mmw phased arrays. In 2018 IEEE International Symposium on Antennas and Propagation USNC/URSI National Radio Science Meeting, pages 627-628, July 2018.

[Sen55] S. Sensiper. Electromagnetic wave propagation on helical structures (a review and survey of recent progress). Proceedings of the IRE, 43(2):149-161, Feb 1955. 
[SGC19] J. Shim, J. Go, and J. Chung. A 1-d tightly coupled dipole array for broadband mmwave communication. IEEE Access, 7:8258-8265, 2019 .

[SJL $\left.{ }^{+} 14\right] \quad$ A. B. Smolders, U. Johannsen, M. Liu, Y. Yu, and P. G. M. Baltus. Differential $60 \mathrm{ghz}$ antenna-on-chip in mainstream $65 \mathrm{~nm}$ cmos technology. In 2014 IEEE Antennas and Propagation Society International Symposium (APSURSI), pages 356-357, July 2014.

[SL17]

N. Saputra and J.R. Long. FM-UWB Transceivers for Autonomous Wireless Systems. River Publishers Series in Circuits and Systems. River Publishers, 2017.

[SMM15] M. N. Shakib, M. Moghavvemi, and W. N. L. Mahadi. A low-profile patch antenna for ultrawideband application. IEEE Antennas and Wireless Propagation Letters, 14:1790-1793, 2015.

[spe] United states frequency allocation chart.

[Spi16] Silvian Spiridon. Toward 5G software defined radio receiver frontends. Springer, 2016.

[Tay94] J.D. Taylor. Introduction to Ultra-Wideband Radar Systems. Taylor \& Francis, 1994.

$\left[\mathrm{THE}^{+} 05\right] \quad$ G. C. Tavik, C. L. Hilterbrick, J. B. Evins, James J. Alter, J. G. Crnkovich, J. W. de Graaf, Wilhelm Habicht, G. P. Hrin, S. A. Lessin, Danke Wu, and S. M. Hagewood. The advanced multifunction RF concept. IEEE Transactions on Microwave Theory and Techniques, 53:1009-1020, 2005.

[TSV11] I. Tzanidis, K. Sertel, and J. L. Volakis. Interwoven spiral array (ispa) with a 10:1 bandwidth on a ground plane. IEEE Antennas and Wireless Propagation Letters, 10:115-118, 2011.

[TSV12] I. Tzanidis, K. Sertel, and J. L. Volakis. Characteristic excitation taper for ultrawideband tightly coupled antenna arrays. IEEE Transactions on Antennas and Propagation, 60(4):1777-1784, April 2012.

[TSV13] I. Tzanidis, K. Sertel, and J. L. Volakis. Uwb low-profile tightly coupled dipole array with integrated balun and edge terminations. 
IEEE Transactions on Antennas and Propagation, 61(6):3017-3025, June 2013.

[Tza11] I. Tzanidis. Ultrawideband low-profile arrays of tightly coupled antenna elements: Excitation, termination and feeding methods. $\mathrm{PhD}$ thesis, Ph.D. dissertation, Dept. Elect. Comput. Eng., Ohio State Univ., Columbus, OH, USA, 2011.

[VAAV17] S. B. Venkatakrishnan, A. Akhiyat, E. A. Alwan, and J. L. Volakis. Multiband and multibeam direction of arrival estimation using onsite coding digital beamformer. IEEE Antennas and Wireless Propagation Letters, 16:2332-2335, 2017.

[VAV18] S. B. Venkatakrishnan, E. A. Alwan, and J. L. Volakis. Wideband RF self-interference cancellation circuit for phased array simultaneous transmit and receive systems. IEEE Access, 6(99):3425-3432, 2018 .

[Vog10] T.R. Vogler. Analysis of the radiation mechanisms in and design of tightly-coupled antenna arrays. PhD thesis, Ph.D. dissertation, Dept. Elect. Computer Eng., Virginia Tech, Blacksburg, VA, USA, 2010.

[Vol07] J. Volakis. Antenna Engineering Handbook, Fourth Edition. McGraw-Hill Companies,Incorporated, 2007.

$\left[\mathrm{VPA}^{+} 17\right] \quad$ S. Bojja Venkatakrishnan, D. K. Papantonis, A. A. Akhiyat, E. A. Alwan, and J. L. Volakis. Experimental validation of on-site coding digital beamformer with ultra-wideband antenna arrays. IEEE Transactions on Microwave Theory and Techniques, 65(11):44084417, Nov 2017.

[Wah09] Mashury Wahab. Radar Radome and its Design Considerations. pages $1-5,122009$.

[WFQX17] N. Wang, M. Fang, J. Qiu, and L. Xiao. Improved design of balanced antipodal vivaldi for mmw applications. In 2017 IEEE International Symposium on Antennas and Propagation USNC/URSI National Radio Science Meeting, pages 2615-2616, July 2017.

[Whe47] H. A. Wheeler. Fundamental limitations of small antennas. Proceedings of the IRE, 35(12):1479-1484, Dec 1947. 
[Whe64] H. Wheeler. Simple relations derived from a phased array made of an infinite current sheet. In 1964 Antennas and Propagation Society International Symposium, volume 2, pages 157-160, 91964.

[WQY17] Ya-Ru Wang, Shi-Wei Qu, and Shiwen Yang. Ultra-wideband widescan tightly coupled dipole array. In 2017 International Workshop on Electromagnetics: Applications and Student Innovation Competition, pages 171-172, May 2017.

[XN01] Xiaojian Xu and Ram Narayanan. FOPEN SAR imaging using UWB step-frequency and random noise waveforms. Aerospace and Electronic Systems, IEEE Transactions on, 37:1287 - 1300, 112001.

[YGV16] E. Yetisir, N. Ghalichechian, and J. L. Volakis. Ultrawideband array with $70^{\circ}$ scanning using fss superstrate. IEEE Transactions on Antennas and Propagation, 64(10):4256-4265, 102016.

[ZB11] Y. Zhang and A. K. Brown. Octagonal ring antenna for a compact dual-polarized aperture array. IEEE Transactions on Antennas and Propagation, 59(10):3927-3932, Oct 2011.

[ZB18] Y. Zhang and A. K. Brown. A wideband planar aperture array using interconnected crossed rings. IEEE Transactions on Antennas and Propagation, pages 1-1, 2018.

$\left[Z_{L P}^{+} 18\right] \quad$ J. Zhong, C. W. Lee, D. Papantonis, A. Kiourti, and J. L. Volakis. Body-worn 30:1 bandwidth tightly coupled dipole array on conductive textiles. IEEE Antennas and Wireless Propagation Letters, 17(5):723-726, May 2018.

[ZPCV12] J. Zhao, D. Psychoudakis, C. Chen, and J. L. Volakis. Design optimization of a low-profile uwb body-of-revolution monopole antenna. IEEE Transactions on Antennas and Propagation, 60(12):55785586, Dec 2012.

$\left[\mathrm{ZYC}^{+} 18\right] \quad$ H. Zhang, S. Yang, Y. Chen, J. Guo, and Z. Nie. Wideband dualpolarized linear array of tightly coupled elements. IEEE Transactions on Antennas and Propagation, 66(1):476-480, Jan 2018.

[ZYX $\left.{ }^{+} 19\right] \quad$ H. Zhang, S. Yang, S. Xiao, Y. Chen, S. Qu, and J. Hu. Ultrawideband phased antenna arrays based on tightly coupled open folded 
dipoles. IEEE Antennas and Wireless Propagation Letters, pages $1-1,2019$.

[ZZG $\left.{ }^{+} 18\right] \quad$ Y. Zhou, F. Zhu, S. Gao, Q. Luo, L. Wen, Q. Wang, X. Yang, Y. Geng, and Z. Cheng. Tightly coupled array antennas for ultrawideband wireless systems. IEEE Access, 6:61851-61866, 2018. 
VITA

\section{ALEXANDER D. JOHNSON}

MAY 1994

| Born - Fernandina Beach, FL

MAY 2016

B.S. Electrical Engineering

Western New England University

DEC 2017

M.S. Electrical Engineering

The Ohio State University

\begin{tabular}{l|l} 
JAN 2018 - PRESENT & $\begin{array}{l}\text { Graduate Researcher } \\
\text { Florida International University }\end{array}$
\end{tabular}

Major Field of Study: Electrical and Computer Engineering

- UWB Phased Arrays

- Millimeter Wave Systems

- Deployable High Gain Antennas (Origami and Textile Antennas)

- Novel Materials

\section{Publications}

1. J. Zhong, A. Johnson, E. A. Alwan and J. L. Volakis, "Dual-Linear Polarized Phased Array With 9:1 Bandwidth and 60 Scanning Off Broadside," in IEEE Transactions on Antennas and Propagation, vol. 67, no. 3, pp. 1996-2001, March 2019.

2. A. Johnson, E. A. Alwan and J. L. Volakis, "A Dual-Polarized Wideband Tightly Coupled Dipole Array for Airborne Applications," submitted to IET Microwaves, Antennas and Propagation.

3. A. Johnson, J. Zhong, S. Bojja Venkatakrishnan, E. A. Alwan and J. L. Volakis, "Phased Array with Low Angle Scanning for > 50 : 1 Bandwidth," submitted to IEEE Transactions on Antennas and Propagation.

4. A. Johnson, J. Zhong, M Livadaru, S. Bojja Venkatakrishnan, E. A. Alwan and J. L. Volakis, "Tightly Coupled Dipole Array with Balanced Wideband Impedance Transformer (BWIT)," submitted to IEEE Transactions on Antennas and Propagation.

Patents

- "Balanced Wideband Impedance Transformer", Patent No.: US 10.320.088 B1. June 11, 2018. 